Supporting information for:

\title{
Bioinformatics-guided expansion and discovery of graspetides
}

Sangeetha Ramesh ${ }^{\dagger \ddagger}$, Xiaorui Guo ${ }^{\S \ddagger}$, Adam J. DiCaprio ${ }^{\S}$, Ashley M. De Lio ${ }^{\sharp{ }^{\perp}}$, Lonnie A. Harris ${ }^{\S}$, Bryce L. Kille"l, Taras V. Pogorelov ${ }^{\mathrm{t}}{ }^{\bar{T}}$, Douglas A. Mitchell ${ }^{\dagger \ddagger \S *}$

${ }^{\dagger}$ Department of Microbiology, University of Illinois at Urbana-Champaign, 600 South Mathews Avenue, Urbana, Illinois 61801, USA. ${ }^{\ddagger}$ Carl R. Woese Institute for Genomic Biology, 1206 West Gregory Drive, Urbana, Illinois 61801, USA. §Department of Chemistry, 600 South Mathews Avenue, Urbana, Illinois 61801, USA. "School of Chemical Sciences, 505 South Mathews Ave, Urbana, Illinois 61801, USA. ${ }^{2}$ National Center for Supercomputing Applications, 1205 West Clark Street, Urbana, Illinois 61801, USA. "Department of Computer Science, 201 North Goodwin Avenue, Urbana, Illinois 61801, USA. "Center for Biophysics and Quantitative Biology, 600 South Mathews Avenue, Urbana, Illinois 61801, USA. ${ }^{\bar{T}}$ Beckman Institute for Advanced Science and Technology, 405 North Mathews Avenue, Urbana, Illinois 61801, USA.

* Corresponding author:

Douglas A. Mitchell (douglasm@illinois.edu), phone: 1-217-333-1345, fax: 1-217-333-0508 


\section{Table of Contents}

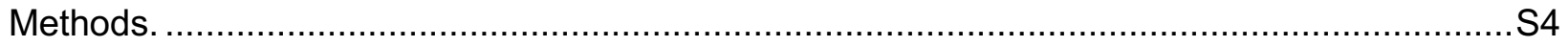

Table S1. Features and weights used for graspetide linear combination scoring. .................S11

Table S2. Graspetide module support vector machine parameters. ................................... 13

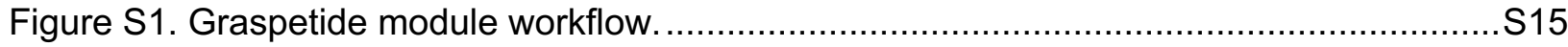

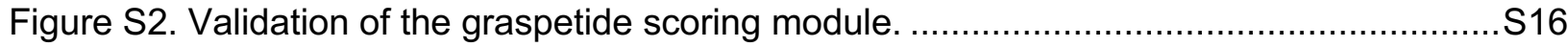

Figure S3. Sequence similarity network of graspetide precursor peptides..........................S17

Table S3. Distribution of graspetide biosynthetic gene clusters. ...................................... 18

Figure S4. Representative graspetide biosynthetic gene clusters from new groups..............S19

Figure S5. Precursor peptide diversity of Grasp-with-Methyltransferase group graspetides....S20

Figure S6. Sequence alignment of SPASM domain-containing proteins.............................S22

Figure S7. Core peptide diversity among Grasp-with-SPASM group graspetides. .................S24

Figure S8. Leader peptide sequences of Grasp-with-SPASM group graspetides..................S25

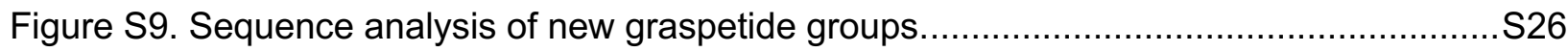

Figure S10. Representative biosynthetic gene cluster for group 15 graspetides. .................S27

Figure S11. Hybrid RiPP BGCs featuring a graspetide synthetase .................................... 28

Figure S12. Graspetide precursor peptide length distribution. .......................................S29

Figure S13 (shown on following two pages). Sequence similarity network of the ATP-grasp

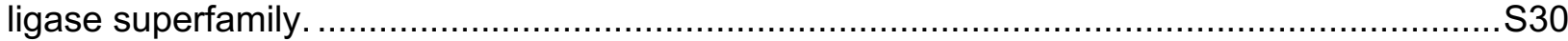

Figure S14. Maximum likelihood tree of graspetide synthetases. ........................................ 33

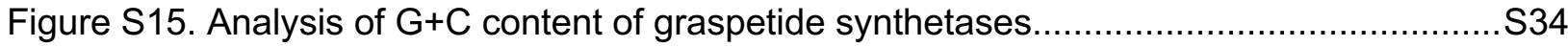

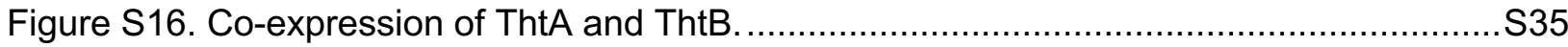

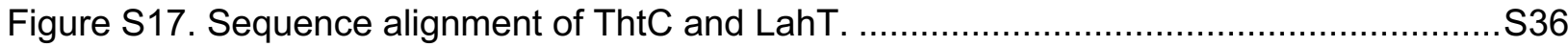

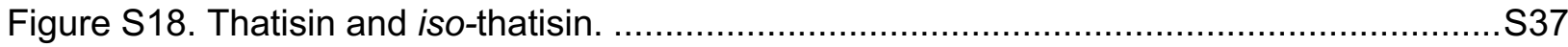

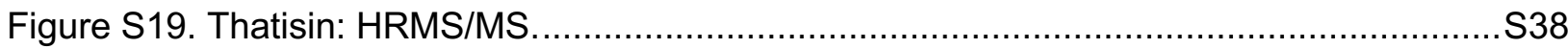

Figure S20. MALDI-TOF-MS analysis of LahT150-digested ThtA-variants..........................S41

Table S4. Summary of thatisin macrolactone variants. ......................................................... 42

Figure S21. Wild-type ThtA-singly modified products: HRMS/MS ......................................S43

Figure S22. ThtA-D3A variant-modified products: HRMS/MS .....................................S47

Figure S23. ThtA-T4A variant- modified products: HRMS/MS. ......................................... 555

Figure S24. ThtA-D14A variant-modified products: HRMS/MS ....................................S61

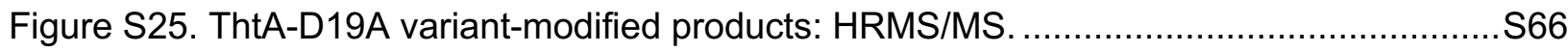

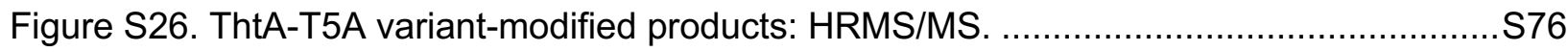

Figure S27. ThtA-T8A variant-modified products: HRMS/MS. ......................................... 885

Figure S28. ThtA-D13A variant-modified products: HRMS/MS .......................................S89 
Figure S29. ThtA-D17A variant-modified products: HRMS/MS ....................................S93

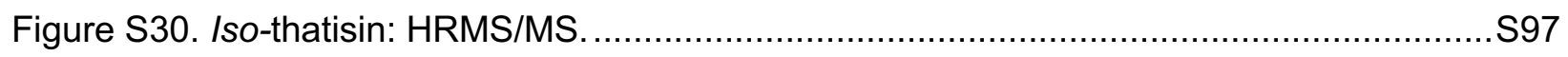

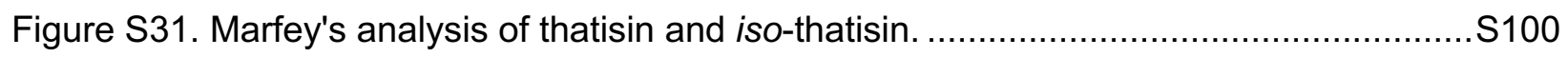

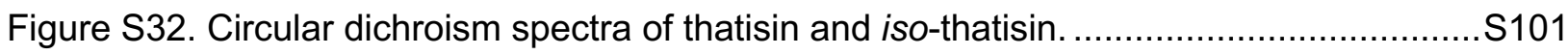

Figure S33. Thatisin conformations based on location of the GPNG bridge. .......................S102

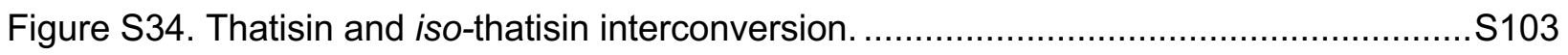

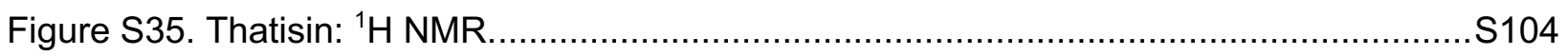

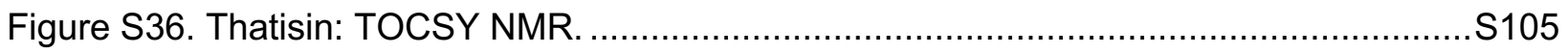

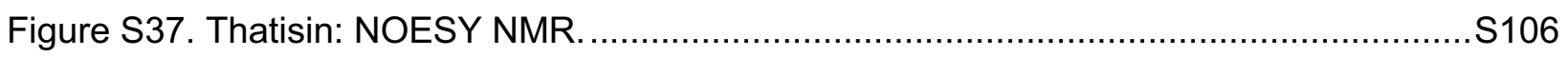

Figure S38. Thatisin: Deuterated Aliphatic TOCSY ................................................... 107

Table S5. NMR chemical shift assignments for thatisin................................................ 108

Figure S39. Thatisin: Pro Assignments in deuterated solvent. ........................................S109

Table S6. Molecular dynamics simulations on 16 possible thatisin conformations. ..............S110

Table S7. Solvent-accessible surface area for $\mathrm{GPNG}_{\text {up }} \mathrm{P} 10_{\text {trans }}-\mathrm{P} 15_{\text {trans }}-\mathrm{P} 18_{\text {trans }}$ structures. .S111

Table S8. Solvent-accessible surface area for $\mathrm{GPNG}_{\text {up }} \mathrm{P} 10_{\text {trans }}-\mathrm{P} 15_{\text {cis }}-\mathrm{P} 18_{\text {trans }}$ structures.....S112 Table S9. Solvent-accessible surface area for $\mathrm{GPNG}_{\text {down }} \mathrm{P} 10_{\text {trans }}-\mathrm{P} 15_{\text {trans }}-\mathrm{P} 18_{\text {trans }}$ structures.

Table S10. Solvent-accessible surface area for $\mathrm{GPNG}_{\text {down }} \mathrm{P} 10_{\text {trans }}-\mathrm{P} 15_{\text {trans }}-\mathrm{P} 18_{\text {cis }}$ structures.

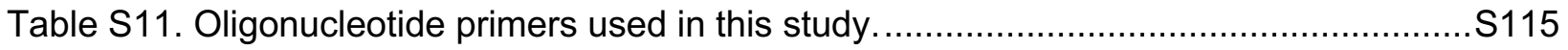

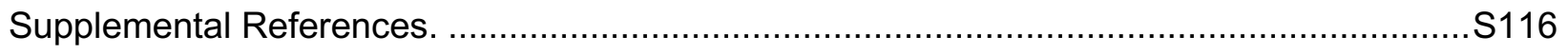




\section{Methods.}

General. Materials and reagents were purchased from Fisher Scientific, Sigma Aldrich, VWR International, or Gold Biotechnology unless otherwise noted. Tryptone was purchased from Research Products International. Molecular biology reagents for cloning (e.g., Q5 polymerase, Gibson Assembly master mix, and deoxynucleotides) were purchased from New England Biolabs. DNA spin columns were procured from Epoch Life Sciences. Oligonucleotide primers for PCR amplification were obtained from Integrated DNA Technologies. PCRs were performed using a Bio-Rad $\mathrm{S} 1000$ thermal cycler. DNA sequencing was performed by the Roy J. Carver Biotechnology Center (University of Illinois at Urbana-Champaign). E. coli DH5a and BL21(DE3)RIPL strains were utilized for plasmid maintenance and overexpression, respectively. The LahT150 expression construct was a gift from Prof. Wilfred A. van der Donk (University of Illinois at Urbana-Champaign). Matrix-assisted laser desorption/ionization time-of-flight mass spectrometry (MALDI-TOF-MS) analysis was performed using an UltrafleXtreme MALDI-TOFTOF mass spectrometer (Bruker Daltonics) in reflector positive mode at the University of Illinois School of Chemical Sciences Mass Spectrometry Laboratory.

Bioinformatic mining and analysis of graspetide biosynthetic gene clusters (BGCs). New release of RODEO. Several improvements have been made to the general Rapid ORF Description and Evaluation Online (RODEO) workflow since its initial release. ${ }^{1}$ RODEO is a pipeline of individual workflows tailored for RiPP genome mining. In previous versions ${ }^{1,2}$, creating a new RiPP scoring module involved changing the logic in multiple workflows, not just the new scoring module. In this updated version, the code has been reconfigured in a way that decouples the RiPP class scoring module from the other essential workflows, streamlining the integration of new class-specific RiPP scoring modules. Additionally, the current version of RODEO has been updated to be compatible with Python 3, given that Python 2 is no longer supported. Currently, RODEO automates the identification of precursor peptides for the following RiPP classes: lanthipeptides, lasso peptides, linaridins, pyritides (which encompasses thiopeptides), ranthipeptides, sactipeptides, and herein graspetides. ${ }^{1-5}$ Additionally, the RiPP genome-mining tool, RRE-Finder, has been integrated. ${ }^{6}$ RODEO is available both as a webtool (http://rodeo.scs.illinois.edu/) and a downloadable command-line tool (https://github.com/themitchell-lab/rodeo2).

Graspetide module. To develop a module for graspetide precursor peptide prediction in RODEO, we generated a set of parameters for heuristic scoring (Table S1) and support vector machine (SVM) classification (Table S2) based on trends observed among characterized and highconfidence, predicted (groups 1-6, previously referred to as omega ester-containing peptides [OEP1-6]) graspetides. ${ }^{7,8}$ Sequence motifs conserved among characterized graspetide precursor peptides identified using the MEME suite ${ }^{9}$ were also part of the scoring metrics (Table S1). Custom profile Hidden Markov Models ( $\mathrm{HHMMs)} \mathrm{generated} \mathrm{using} \mathrm{HMMER3} \mathrm{were} \mathrm{integrated} \mathrm{to}$ readily distinguish graspetide-associated ATP-grasp ligases (graspetide synthetases, henceforth) from those involved in other biochemical processes. ${ }^{10}$ Seven custom pHMMs were generated for graspetide synthetases associated with each characterized graspetide group (Supplementary Dataset 1). To detect graspetide precursor peptides with multiple repeat sequences (which may otherwise bypass any RiPP scoring module owing to length restrictions imposed by RODEO), the Rapid Automatic Detection and Alignment of Repeats in protein sequences (RADAR) package ${ }^{11}$ 
was integrated into the graspetide scoring module. The SVM was trained using a dataset of 715 positives and 64,833 negatives. The positive dataset included randomly selected, characterized, and high-confidence predicted graspetide precursor peptides from groups 1-6. ${ }^{8}$ The negative dataset was comprised of hypothetical non-coding sequences obtained via 6-frame translation of the intergenic region. While several RiPP scoring modules in RODEO support the prediction of the leader peptide cleavage site ${ }^{1,3-5}$, the graspetide module does not, owing to the current inability to accurately predict such sites based on available data.

The graspetide module was tested using a set of predicted precursor peptides from graspetide groups with characterized members. ${ }^{8} \mathrm{~A}$ plot between the prediction accuracy and the cut-off score of the test set was used to determine the positive cut-off score as 12 for graspetide precursor peptide prediction (Figure S2).

Bioinformatic analysis of graspetide BGCs. Three iterations of Position-Specific Iterative BLAST ${ }^{12}$ searching were performed (January 2021) using the following protein queries: MvdC (WP_042156022.1) and MvdD (WP_042156020.1) from the microviridin K BGC, PsnB (WP_006971586.1) from the plesiocin BGC, TgnB (WP_000849148.1) from the thuringinin BGC, OJW02008.1 from OEP4-1, GAJ78971.1 from OEP5-1, and WP_059136627.1 from OEP6-1. The expectation value cut-off was set to $\mathrm{e}^{-30}$ using the NCBI non-redundant database. The complete list of putative ATP-grasp ligases was then used as input for analysis using the graspetide module of RODEO and later curated to generate a list of putative graspetide precursor peptides.

Phylogenetic distribution analysis was performed on the ATP-grasp enzymes that RODEO judged as occurring within a valid graspetide BGC. All sequence similarity networks (SSNs) were constructed using the Enzyme Function Initiative Enzyme Similarity Tool (EFI-EST) (https://efi.igb.illinois.edu/efi-est/) and visualized using Cytoscape. ${ }^{13-15}$ For analysis of the ATPgrasp ligase superfamily, bacterial and archeal representative sequences from InterPro family IPR011761 were obtained from the UniRef50 database. ${ }^{16}$ Graspetide synthetases were introduced into this dataset since the UniProt database lacks most members (RODEO analysis was performed using the NCBI RefSeq database). The maximum likelihood tree analysis was performed by aligning sequences using Multiple Sequence Alignment based on fast Fourier Transform (MAFFT version 7.271) ${ }^{17}$ with the L-INS-i alignment option and transformed into a maximum likelihood tree using FastTree $2.15^{18}$ with the default Jones-Taylor-Thornton model. The tree was visualized using the Interactive Tree of Life (iTOL) ${ }^{19}$ website (https://itol.embl.de/). All sequence logos were generated using WebLogo (http://weblogo.threeplusone.com/) ${ }^{20}$

Genomic DNA extraction. Lysobacter antibioticus ATCC 29479 was grown overnight in $5 \mathrm{~mL}$ nutrient broth [beef extract $3 \mathrm{~g} / \mathrm{L}$, peptone $5 \mathrm{~g} / \mathrm{L}, \mathrm{pH} 6.8$ ] at $30^{\circ} \mathrm{C}$. Genomic DNA was isolated using the DNAeasy UltraClean Microbial Kit (Qiagen) per the manufacturer's instructions.

Cloning of thatisin and variant generation. ThtA (WP_057916646.1), and the cognate graspetide synthetase, ThtB (WP_057916645.1) from L. antibioticus ATCC 29479 were cloned into a modified pETDuet-1 vector (pETDuetK_MBP, henceforth). pETDuetK_MBP was produced by introducing a maltose-binding protein (MBP) tag with a tobacco etch virus (TEV) proteasecleavable linker to the N-terminus of the protein encoded in multiple cloning site (MCS) 1. Additionally, the ampicillin-resistance gene was replaced with a kanamycin resistance gene from pRSF-Duet. The genes encoding ThtA and ThtB were PCR amplified using Q5 polymerase with 
the oligonucleotide primers listed in Table S11. After product confirmation by gel electrophoresis, the properly sized DNA was subjected to gel extraction (QIAquick gel extraction kit, Qiagen) and cloned into the pETDuetK_MBP vector by Gibson assembly. ThtA and thtB were cloned into the MCS 1 and 2, respectively. The resulting construct, pETDuetK_MBP-ThtA_ThtB, was used for the co-expression experiments. The expression plasmid to obtain unmodified MBP-ThtA was similarly constructed but contained an empty MCS 2. All ThtA-variants were produced by Gibson assembly using pETDuetK_MBP-ThtA_ThtB as the template. Primers are listed in Table S11.

\section{Expression and purification of thatisin and variants thereof.}

All procedures, unless otherwise noted, were performed in an identical manner for both wild-type thatisin and thatisin variants.

Expression. E. coli BL21(DE3)-RIPL were transformed with pETDuetK_MBP-ThtA (control), pETDuetK_MBP-ThtA_ThtB, or the precursor peptide variant being analyzed (e.g., pETDuetK_MBP-ThtA-D3A_ThtB) and plated on Luria-Bertani (LB) agar medium supplemented with $50 \mu \mathrm{g} / \mathrm{mL}$ kanamycin and $34 \mu \mathrm{g} / \mathrm{mL}$ chloramphenicol. A single colony was inoculated into 10 $\mathrm{mL}$ of LB broth supplemented with the same antibiotics and grown overnight at $37^{\circ} \mathrm{C}$. This starter culture was used to inoculate $1 \mathrm{~L}$ Terrific Broth (TB) in a $4 \mathrm{~L}$ flat bottom flask and grown at $37^{\circ} \mathrm{C}$ and 220 rpm shaking to an $\mathrm{OD}_{600}$ of 1.2. Expression was then induced with $0.5 \mathrm{mM}$ isopropyl $\beta$ D-1thiogalactopyranoside for $18 \mathrm{~h}$ at $22{ }^{\circ} \mathrm{C}$ and $220 \mathrm{rpm}$ shaking. After expression, cells were harvested by centrifugation at $4,000 \times \mathrm{g}$, washed with phosphate buffered saline, and the harvested cells were frozen at $-20{ }^{\circ} \mathrm{C}$ until purification.

Purification by amylose affinity chromatography. The harvested cells were resuspended in icecold lysis buffer [50 mM Tris pH 7.5, $500 \mathrm{mM} \mathrm{NaCl}, 2.5 \%(\mathrm{v} / \mathrm{v})$ glycerol, $0.1 \%(\mathrm{v} / \mathrm{v})$ Triton X-100 supplemented with $3.5 \mathrm{mg} / \mathrm{mL}$ lysozyme and a $0.5 \mathrm{~mL}$ protease inhibitor cocktail $(16 \mathrm{mg} / \mathrm{mL}$ benzamidine $\mathrm{HCl}, 6 \mathrm{mM}$ phenylmethylsulfonyl fluoride (PMSF), $0.1 \mathrm{mM}$ leupeptin, $0.1 \mathrm{mM} \mathrm{E64)]}$ and gently rocked at $4{ }^{\circ} \mathrm{C}$ for $30 \mathrm{~min}$. The samples were then subjected to three rounds of sonication at $60 \%$ amplitude for $45 \mathrm{~s}$ with a $10 \mathrm{~min}$ cooling period between each round. The cellular debris was separated by centrifugation at $20,000 \times \mathrm{g}$ for $1 \mathrm{~h}$ at $4{ }^{\circ} \mathrm{C}$. The clarified cell lysate was supplied by gravity flow to a column packed with amylose resin pre-equilibrated with ice-cold lysis buffer. The column was then washed with $2 \times$ column volumes of wash buffer [50 $\mathrm{mM}$ Tris $\mathrm{pH} 7.5,400 \mathrm{mM} \mathrm{NaCl}, 2.5 \%(\mathrm{v} / \mathrm{v})$ glycerol] and eluted using elution buffer [50 mM Tris $\mathrm{pH} 7.5,150 \mathrm{mM} \mathrm{NaCl}, 10 \mathrm{mM}$ maltose, 2.5\% ( $/ \mathrm{v})$ glycerol]. Eluted MBP-ThtA was then concentrated using a $30 \mathrm{kDa}$ molecular weight cut-off (MWCO) Amicon Ultra $15 \mathrm{~mL}$ centrifugal filter. A 100-fold buffer exchange was then performed with either storage buffer [50 mM HEPES $\mathrm{pH} 7.5,150 \mathrm{mM} \mathrm{NaCl}, 2.5 \%(\mathrm{~V} / \mathrm{V})$ glycerol] or $10 \mathrm{mM}$ aq. $\mathrm{NH}_{4} \mathrm{HCO}_{3} \mathrm{pH} 7.5$ to a final concentration of $50-70 \mathrm{mg} / \mathrm{mL}$ for subsequent proteolysis by TEV protease or LahT150. MBP-ThtA concentrations were estimated using A280 measurement with the extinction coefficients calculated using Expasy ProtParam: https://web.expasy.org/protparam.

Removal of MBP and leader peptide. For initial MALDI-TOF-MS analysis, MBP was removed using TEV protease while the cognate leader peptide was removed using LahT150. ${ }^{21}$ For ring connectivity determination by high-resolution and tandem mass spectrometry (HRMS/MS), the 
MBP-tag along with the leader peptide was removed from modified ThtA (or variants) by an optimized protocol using only LahT150.

MBP-ThtA samples $(400 \mu \mathrm{M})$ were digested using TEV protease $(40 \mu \mathrm{M})$ overnight at $25{ }^{\circ} \mathrm{C}$ to remove MBP. Subsequently, the solution was subjected to a Ni-NTA affinity column to remove $\mathrm{MBP}$, as the protein is also $\mathrm{N}$-terminally His-tagged. The flow-through containing ThtA was collected. The column was washed with $50 \mathrm{mM}$ Tris $\mathrm{pH} 8$ at $25^{\circ} \mathrm{C}$, and the flow-through was collected until no ThtA was detectable by MALDI-TOF-MS. The flow-through was concentrated using a $3 \mathrm{kDa}$ MWCO Amicon Ultra $15 \mathrm{~mL}$ centrifugal filter. The sample containing the precursor peptide was digested using LahT150 (40 $\mu \mathrm{M}$; purified using published protocols $\left.{ }^{21}\right)$ at $25{ }^{\circ} \mathrm{C}$ overnight to remove the leader peptide.

The MBP-tagged leader peptide was removed from $1.5 \mathrm{mM}$ MBP-ThtA (or variants) using $150 \mu \mathrm{M}$ LahT150 at $25^{\circ} \mathrm{C}$ overnight in $10 \mathrm{mM}$ aq. $\mathrm{NH}_{4} \mathrm{HCO}_{3} \mathrm{pH}$. The digested sample was subjected to filtration using a $10 \mathrm{kDa}$ MWCO Amicon Ultra $15 \mathrm{~mL}$ centrifugal filter. While the MBP-tagged leader peptide and LahT150 protein were retained in the filtrate, the modified core peptide was found in the flow-through. The filtrate was resuspended in $10 \mathrm{mM}$ aq. $\mathrm{NH}_{4} \mathrm{HCO}_{3} \mathrm{pH} 8$ and further filtered to collect flow-through until no residual core peptide in the filtrate was identified by MALDITOF-MS.

HPLC purification. MeCN was added to Amicon-filtered modified ThtA core peptide (or variant) dissolved in $10 \mathrm{mM}$ aq. $\mathrm{NH}_{4} \mathrm{HCO}_{3}$ until a final ratio of $80 / 2010 \mathrm{mM}$ aq. $\mathrm{NH}_{4} \mathrm{HCO}_{3} / \mathrm{MeCN}$. This solution was subsequently $0.22 \mu \mathrm{m}$ syringe filtered (BD technologies) before purification by highpressure liquid chromatography (HPLC) using a Perkin Elmer Flexar instrument equipped with a Macherey-Nagel Nucleosil C18 column $(250 \times 10 \mathrm{~mm}, 5 \mu \mathrm{m}$ particle size, $100 \AA$ pore size $)$ at a flow rate of $1 \mathrm{~mL} / \mathrm{min}$. Dissolved thatisin (or variant) was injected with a mobile phase of $10 \mathrm{mM}$ aq. $\mathrm{NH}_{4} \mathrm{HCO}_{3} / \mathrm{MeCN}$ with a gradient from $5-20 \% \mathrm{MeCN}$ over $60 \mathrm{~min}, 20-90 \% \mathrm{MeCN}$ over $5 \mathrm{~min}$, hold at $90 \% \mathrm{MeCN}$ for 5 minutes, followed by $90-5 \% \mathrm{MeCN}$ over 5 minutes. Absorbance was monitored at 220 and $280 \mathrm{~nm}$. Fractions containing ThtA core peptide at various modification stages were collected and lyophilized for subsequent analyses.

MALDI-TOF-MS analysis. MALDI-TOF-MS was used to monitor post-translational modification, proteolysis, and methanolysis. Thatisin (or variant) and related peptides at various modification stages were crystallized for MALDI-TOF-MS analysis using $20 \mathrm{mg} / \mathrm{mL}$ of a-cyano-4hydroxycinnamic acid and $10 \mathrm{mg} / \mathrm{mL}$ 2,5-dihydroxybenzoic acid in $70 \% \mathrm{MeCN}$.

Circular Dichroism (CD) spectroscopy. CD spectra were acquired using a JASCO spectropolarimeter J-715 with Peltier temperature control. Lyophilized sample (thatisin and isothatisin) was resuspended in water to $300 \mu \mathrm{M}$ immediately prior to data acquisition. Spectra were recorded from 260 to $200 \mathrm{~nm}$ at a scan rate of $200 \mathrm{~nm} / \mathrm{min}$ at $0.1 \mathrm{~nm}$ resolution at $10{ }^{\circ} \mathrm{C}$. The data shown are the average of three acquisitions.

Methanolysis. Methanolysis was performed on lyophilized, HPLC-purified samples using 100 $\mathrm{mM}$ sodium methoxide in methanol $(\mathrm{pH}>11)$. Samples were reacted for 10 min for partial methanolysis and $1 \mathrm{~h}$ for complete methanolysis prior to quenching with $350 \mu \mathrm{l} 10 \mathrm{M}$ acetic acid. Methanolized samples were desalted using C18 ZipTips (EMD Millipore) and eluted into 75\% aq. MeCN for MS analysis. 
HRMS/MS. HPLC-purified, lyophilized samples was resuspended in ESI mix [ $80 \% \mathrm{MeCN}, 19 \%$ $\mathrm{H}_{2} \mathrm{O}, 1 \%$ acetic acid]. Methanolized samples were desalted using ZipTips, eluted into $75 \%$ aq. $\mathrm{MeCN}$, and diluted 1:1 in ESI mix before HRMS/MS analysis. Samples were directly infused onto a ThermoFisher Scientific Orbitrap Fusion ESI-MS using an Advion TriVersa Nanomate 100. MS calibration was performed with Pierce LTQ Velos ESI Positive Ion Calibration Solution (ThermoFisher). The MS was operated using the following parameters: 100,000 resolution, $1 \mathrm{~m} / \mathrm{z}$ isolation width (MS/MS), 35 normalized collision energy (MS/MS), 0.4 activation q value (MS/MS), and $30 \mathrm{~ms}$ activation time (MS/MS). Fragmentation was performed using collision-induced dissociation (CID) at $30 \%$ and $70 \%$. Data analysis was conducted using the Qualbrowser application of Xcalibur software (ThermoFisher Scientific).

Marfey's analysis of thatisin and iso-thatisin. Thatisin/iso-thatisin $(300 \mu \mathrm{g})$ was dissolved in $200 \mu \mathrm{L} 6 \mathrm{M} \mathrm{DCl}$ in $\mathrm{D}_{2} \mathrm{O}\left(35 \% \mathrm{w} / \mathrm{v} \mathrm{DCl}\right.$ diluted $1: 2$ into $\mathrm{D}_{2} \mathrm{O}$, Aldrich and Cambridge Isotope Laboratory, respectively). The solution was transferred to a $5 \mathrm{~mL}$ pear-shaped-flask, sealed with a septum. The solution was then subjected to two freeze-pump-thaw cycles and heated to 100 ${ }^{\circ} \mathrm{C}$ using a temperature-controlled heating block (Optimag-ST, Chemglass Life Sciences) for $4 \mathrm{~h}$ under reduced pressure. The solution was then dried using a Speedvac concentrator (Savant ISS110).

The resulting hydrolysate was dissolved in $150 \mu \mathrm{L} 1 \mathrm{M} \mathrm{NaHCO}_{3}$ and mixed with $150 \mu \mathrm{L} 4 \mathrm{mg} / \mathrm{mL}$ or $12 \mathrm{mg} / \mathrm{mL}$ 1-fluoro-2-4-dinitrophenyl-5-L-alanine amide (FDAA, Thermo Scientific). The higher concentration of FDAA was used to aid analysis by modifying both amines of lysine. The reaction mixtures were heated to $60{ }^{\circ} \mathrm{C}$ for $2 \mathrm{~h}(4 \mathrm{mg} / \mathrm{mL}$ FDAA $)$ or $4 \mathrm{~h}(12 \mathrm{mg} / \mathrm{mL}$ FDAA $)$ and quenched dropwise using $30 \mu \mathrm{L} 6 \mathrm{M} \mathrm{HCl}$, and dried using a Speedvac concentrator. Amino acid standards were made by drying down $200 \mu \mathrm{L}$ of a commercial L-amino acid solution $(0.5 \mu \mathrm{mol} / \mathrm{mL})$ and reacted with FDAA as above. Rather than purchasing a D-amino acid standard, fluoro-2-4dinitrophenyl-5-D-alanine amide (D-FDAA, Toronto Research Chemicals) was reacted with the Lamino acid standard. The resulting adduct is the enantiomer of a D-amino acid-FDAA adduct, and thus indistinguishable via HPLC on an achiral stationary phase.

The resulting derivatized L-/D-amino acid standards and (iso-)thatisin hydrolysates were reconstituted in $300 \mu \mathrm{L} 15 \%$ aq. MeCN $+0.1 \%$ formic acid (LS-MS grade), centrifuged to remove particulates, and filtered with a $0.45 \mu \mathrm{m}$ centrifugal filter (Thermo Scientific). LC-MS analysis was performed on a Shimadzu LC-MS 2020 with a quadrapole detector and a Macherey-Nagel Nucleodor C18 column $(250 \times 4.6 \mathrm{~mm}, 5 \mu \mathrm{m}$ particle size, $100 \AA$ pore size) The samples were analyzed in batch under the following conditions: solvent $\mathrm{A} 0.1 \%$ aq. formic acid, solvent $\mathrm{B}$ MeCN $+0.1 \%$ formic acid, $1 \mathrm{~mL} / \mathrm{min}$ flow rate, and the following gradient: $15 \%$ B for $7 \mathrm{~min}, 15-50 \% \mathrm{~B}$ over $45 \mathrm{~min}$, hold at $50 \%$ B for $5 \mathrm{~min} .20 \mu \mathrm{L}$ of analyte was injected and the $[\mathrm{M}+\mathrm{H}]^{+}$ions of the amino acid-Marfey's reagent adducts were selectively monitored and are as follows $(\mathrm{m} / \mathrm{z})$ : Lys (doubly modified), 651; Ala, 342; Asp, 386; Pro, 368; Thr, 372; Phe, 418; His, 408; Leu, 384.

Nuclear Magnetic Resonance (NMR) Spectroscopy. For NMR structure elucidation ca. $2.1 \mathrm{mg}$ of thatisin was dissolved in $290 \mu \mathrm{L}$ of either ice-cold 9:1 $\mathrm{H}_{2} \mathrm{O}: \mathrm{D}_{2} \mathrm{O}$ (Sigma Aldrich, 99.9\% atom D) or ice-cold $\mathrm{D}_{2} \mathrm{O}$ and clarified by centrifugation at $25^{\circ} \mathrm{C}$ for $5 \mathrm{~min}$ at $17,000 \mathrm{xg}$. The supernatant was removed and placed in a $5 \mathrm{~mm} \mathrm{D}_{2} \mathrm{O}$-matched Shigemi tube (Millipore-Sigma). Conformational exchange was prevented by acquiring all experimental data at $12.0^{\circ} \mathrm{C}$. Variable temperature control was calibrated using the $\Delta \mathrm{ppm}$ between the $-\mathrm{CH}_{3}$ and $-\mathrm{OH}$ resonances of $100 \%$ methanol. Experiments were performed on a $750 \mathrm{MHz}$ Agilent VNMRS narrow-bore NMR operating at 
749.375 MHz equipped with a triple resonance ${ }^{1} \mathrm{H}-{ }^{13} \mathrm{C} /{ }^{15} \mathrm{~N}$ Varian probe with gradient and pulseshaping capabilities. Data was recorded on a console operating VnmrJ 3.2A. Standard Varian Biopack pulse sequences were used for all acquisitions. Mixing times for ${ }^{1} \mathrm{H}-{ }^{1} \mathrm{H}$ TOCSY and ${ }^{1} \mathrm{H}-$ ${ }^{1} \mathrm{H}$ NOESY experiments were set to $70 \mathrm{~ms}$ and $300 \mathrm{~ms}$, respectively. All spectra were collected with 96 increments in the indirect dimension under WET solvent suppression. Deuterated samples were studied using ${ }^{1} \mathrm{H}-{ }^{1} \mathrm{H}$ TOCSY and ${ }^{1} \mathrm{H}-{ }^{1} \mathrm{H}$ NOESY with mixing times of $60 \mathrm{~ms}$ and $400 \mathrm{~ms}$, respectively.

Data was converted and processed in NMRPipe. ${ }^{22}$ Spectra were referenced to the chemical shift of water (4.77 ppm). Spectra were phase- and baseline-corrected and subject to linear prediction. Spectra were analyzed and assigned in NMRFAM-SPARKY. ${ }^{23}$ Assigned NOE contacts were converted to peak intensities and imported into Microsoft Excel for further calculation. Contacts were calibrated with the interproton distance of $2.51 \AA$ of $\mathrm{Thr} 8 \mathrm{H}_{\alpha}-\mathrm{H}_{\mathrm{N}}$ using eq. 1.

$$
{\frac{V_{A B}}{r_{A B}}}^{-6}={\frac{V_{B C}}{r_{B C}}}^{-6}
$$

Where $\mathrm{V}$ is the measured NOE volume, and $\mathrm{r}$ is the interproton distance. The aforementioned NOE calibrant was used in place of $r A B$.

Growth suppression assays. Growth suppression activity of thatisin was tested using the broth microdilution method against the following strains: Staphylococcus aureus USA300, Enterococcus faecalis ATCC 19433, Pseudomonas aeruginosa PA01, Klebsiella pneumoniae ATCC 27736, Escherichia coli K12 MC4100, Micrococcus luteus DSM 1790. Brain heart infusion (BHI, BD Biosciences) was used as the growth medium for all strains except $K$. pneumoniae which was grown in LB. The strains were grown overnight in $5 \mathrm{~mL}$ of appropriate media at $37{ }^{\circ} \mathrm{C}$ (exception: $30{ }^{\circ} \mathrm{C}$ for $\mathrm{M}$. luteus) to stationary phase. Cultures were diluted 500 -fold using the appropriate medium and grown to an $\mathrm{OD}_{600}$ of 0.5 . Cultures were diluted further by 50,000 -fold to obtain $\sim 1 \times 10^{5} \mathrm{CFU} / \mathrm{mL}$ and were added to a 96-well plate. Thatisin $(0.125-64 \mu \mathrm{g} / \mathrm{mL}$ in $97 \% \mathrm{BHI}$ and $\left.3 \% \mathrm{H}_{2} \mathrm{O} v / v\right)$ was added in successive 2-fold serial dilution to the cultures. Gentamicin (0.125$64 \mu \mathrm{g} / \mathrm{mL}$ ) was used as the positive control for all strains except $M$. luteus, for which chloramphenicol $(0.125-64 \mu \mathrm{g} / \mathrm{mL})$ was used. Negative controls lacking thatisin and antibiotics were also used. The plate containing $M$. luteus was incubated overnight at $30{ }^{\circ} \mathrm{C}$ while all other plates were incubated at $37^{\circ} \mathrm{C}$ with $220 \mathrm{rpm}$ shaking.

Protease inhibition assays. The serine proteases tested include $\alpha$-chymotrypsin from bovine pancrease (C3142, Sigma-Aldrich), trypsin from bovine pancreas (T1426, Sigma-Aldrich), and elastase from porcine pancreas (V1891, Promega). Their corresponding $p$-nitroanilide substrates are Na-benzoyl-DL-arginine $p$-nitroanilide hydrochloride (BAPNA; B4875, Sigma-Aldrich), $N$ succinyl-Ala-Ala-Pro-Phe $p$-nitroanilide ( $N$-succinyl-Ala-Ala-Pro-Phe-pNA; S7388, Sigma Aldrich) and $N$-succinyl-Ala-Ala-Ala $p$-nitroanilide ( $N$-succinyl-tri-Ala-pNA, Sigma Aldrich S4760). The assay buffer was $50 \mathrm{mM}$ Tris- $\mathrm{HCl}, 20 \mathrm{mM} \mathrm{CaCl} 2 \mathrm{pH}$ 7.5. The proteases were dissolved in the assay buffer to a stock concentration of $4 \mu \mathrm{M}$ (trypsin) or $0.4 \mu \mathrm{M}$ (chymotrypsin and elastase). The substrates were dissolved in the assay buffer to a stock concentration of $400 \mu \mathrm{M}$ (BAPNA in $0.35 \% \mathrm{v} / \mathrm{V}$ DMSO and N-succinyl-tri-Ala-pNA) or $200 \mu \mathrm{M}$ (N-succinyl-Ala-Ala-Pro-Phe-pNA). Protease inhibition assays were performed in 96-well plates. Proteases (final concentration: 200 
$\mathrm{nM}$ trypsin,10 nM chymotrypsin and $20 \mathrm{nM}$ elastase) and buffer were first added to the wells. Thatisin (200-0.002 $\mu \mathrm{M})$ was added in successive 10-fold serial dilution to the wells and incubated for $10 \mathrm{~min}$ at $37^{\circ} \mathrm{C}$ (trypsin and chymotrypsin) or $25^{\circ} \mathrm{C}$ (elastase). Substrates (final concentration: $200 \mu \mathrm{M}$ BAPNA/ $200 \mu \mathrm{M}$ N-succinyl-tri-Ala-pNA/ $100 \mu \mathrm{M}$ N-succinyl-Ala-Ala-Pro-Phe-pNA) were then added to the wells and the $\mathrm{A}_{405}$ was measured immediately using a FilterMax F5 multimode plate reader (Molecular Devices) using the assay buffer as blank. The plate was incubated at 37 ${ }^{\circ} \mathrm{C}$ (trypsin and chymotrypsin) or $25{ }^{\circ} \mathrm{C}$ (elastase) and the $\mathrm{A}_{405}$ was recorded every 10 min for 1 h. PMSF (1000-0.01 $\mu \mathrm{M})$ was used as the positive control. Negative controls lacking thatisin, enzyme or substrate were also used.

\section{Computation.}

Model building and optimization. Two thatisin/iso-thatisin conformers with a GPNG bridge "up" vs. bridge "down" assembly were constructed. From these two conformers, 16 total thatisin conformers ( 8 each for the bridge up and bridge down conformations) were assembled, each by systematically varying cis/trans configuration of the three Pro side chains (Pro10, Pro15, Pro18). Peptide assembly was completed in the Molecular Operating Environment (MOE) version $2019.0102^{24}$ using the protein builder function. After initial assembly, $\mathrm{MOE}^{24}$ was used to perform two different energy minimizations on all conformers. The first energy minimization was performed with the AMBER 14:EHT ${ }^{25}$ force field with a cutoff of $10 \AA$ for non-bonded interactions in a Born ${ }^{26}$ implicit solvent with interior and exterior dielectric constants of 1 and 80 , respectively. A second energy minimization was performed with the same force field in an explicit water solvent with 0.01 $\mathrm{M} \mathrm{NaCl}$ and an RMS gradient of $0.0001 \mathrm{kcal} / \mathrm{mol} . \AA^{2}$. After energy minimization, all 16 conformers were manually verified to maintain the starting Pro geometries.

LowModeMD ${ }^{27}$ conformational search simulations were performed on all 16 conformers with the AMBER14:EHT ${ }^{25}$ force field with a cutoff of $10 \AA$ in a Born ${ }^{26}$ implicit solvent with interior and exterior dielectric constants of 1 and 80 , respectively. The iteration limit was set to 10,000 steps with a rejection limit of 100 . The molecular mechanics iteration limit was set to 500 , and the RMS gradient was set to $0.005 \mathrm{kcal} / \mathrm{mol} . \AA^{2}$. The total possible number of conformation results was limited to 10,000 , with an RMSD limit between unique conformations set to $0.15 \AA$ and an energy cutoff window of 7 . The Pro geometries of the resultant structures from all 16 LowModeMD simulations were examined to observe whether the Pro could switch geometries during the simulation and determine whether some geometries were more populated than others.

Solvent-accessible surface area calculations. The solvent-accessible surface area (SASA) of the carbonyl carbons of Thr5-Asp13 and Thr8-Asp17 macrolactones were performed in Visual Molecular Dynamics ${ }^{28}$ using a probe size of $2 \AA$ to simulate methanol solvent used in the methanolysis experiments. 
Table S1. Features and weights used for graspetide linear combination scoring.

\begin{tabular}{|c|c|c|}
\hline & Feature & Weight \\
\hline \multirow{25}{*}{ 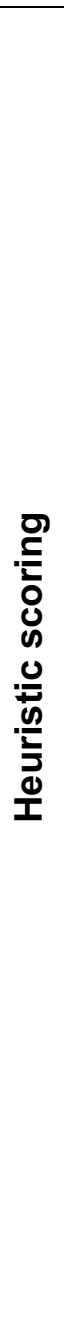 } & Precursor is encoded $<1,100$ nucleotides of ATP-grasp ligase & +1 \\
\hline & Precursor is encoded $<300$ nucleotides of ATP-grasp ligase & +2 \\
\hline & Precursor is encoded $>2,000$ nucleotides of ATP-grasp ligase & -1 \\
\hline & Second half of precursor contains $>8 \%$ Asp residues (acceptor residue) & +1 \\
\hline & Second half of precursor contains $>7 \%$ Thr residues (donor residue) & +1 \\
\hline & Precursor (full-length) contains $>7 \%$ Lys residues (donor residue) & +1 \\
\hline & Second half of precursor contains $>7 \%$ Pro residues & +1 \\
\hline & Precursor contains $>0$ Cys residues & -2 \\
\hline & Second half of precursor contains $<3$ acceptor residues $($ Asp + Glu $)$ & -1 \\
\hline & Second half of precursor contains $<3$ donor residues (Ser + Thr + Lys) & -1 \\
\hline & Second half of precursor contains more donor residues than acceptor residues & +1 \\
\hline & $\begin{array}{l}\% \text { acceptor residues in the second half of precursor }>\% \text { acceptor residues in the } \\
\text { first half of precursor }\end{array}$ & +1 \\
\hline & $\%$ acceptor residues in the second half of precursor $>13 \%$ & +1 \\
\hline & $\%$ donor residues in second half of precursor $>18 \%$ & +1 \\
\hline & Precursor ends with Leu & +2 \\
\hline & Precursor ends with Asp or Val or Ser & +1 \\
\hline & First half of the precursor contains a "PFxL" motif & +1 \\
\hline & Precursor and the ATP-grasp ligase are encoded on the same strand & +1 \\
\hline & $\begin{array}{l}\text { Gene cluster contains one of the following: PF0005, PF06472, PF00664, PF03412, } \\
\text { TIGR03796, TIGR01846, TIGR03797, TIGR00954, TIGR02203, TIGR02204, } \\
\text { TIGR03375 (ABC transporters that co-occur frequently) }\end{array}$ & +1 \\
\hline & $\begin{array}{l}\text { Gene cluster contains one of the following: PF13302, PF00583, PF13523 } \\
\text { (acetyltransferases that co-occur frequently) }\end{array}$ & +1 \\
\hline & A local gene product hits TIGR04188 (methyltransferase) & +1 \\
\hline & $\begin{array}{l}\text { Precursor hits PF12559 (serine endopeptidase inhibitor) or TIGR04186 } \\
\text { (GRASP_targ) }\end{array}$ & +5 \\
\hline & $\begin{array}{l}\text { Precursor hits PF14404 (strep_pep), PF14406 (bacteroid_pep), PF14407 } \\
\text { (frankia_pep), PF14408 (actino_pep), or PF14409 (herpeto_pep) }\end{array}$ & +5 \\
\hline & Acceptor compressionc index $>$ Donor compressionc index ${ }^{*}$ & +1 \\
\hline & $\begin{array}{l}\text { Calculated charge at } \mathrm{pH}=7 \text { of second half of precursor }<\text { charge of first half of } \\
\text { precursor }\end{array}$ & +1 \\
\hline \multirow{9}{*}{$\sum_{\mathbf{+}}^{\underset{\mathbf{W}}{W}}$} & Precursor peptide contains sequence motif \#1 "PFFAx 2 " " & +1 \\
\hline & Precursor peptide contains "n" sequence motif \#2 "TxKxPSDx(E/D)(E/D)" & $+n$ \\
\hline & Precursor peptide contains sequence motif \#3 "LFIx(D/E)L" & +1 \\
\hline & 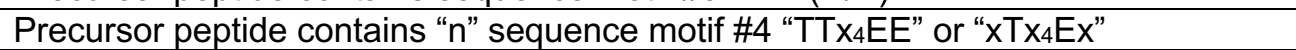 & $+n$ \\
\hline & Precursor peptide contains sequence motif $\# 5$ "KPY $\mathrm{x}_{3} \mathrm{YxE}$ " & +1 \\
\hline & 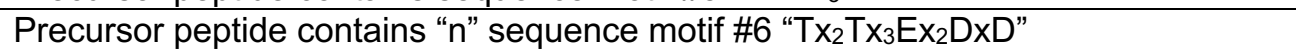 & $+n$ \\
\hline & 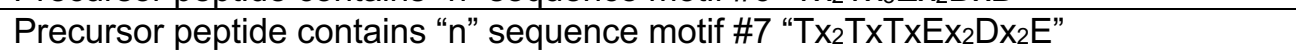 & $+n$ \\
\hline & Precursor peptide contains "n" sequence motif \#8 "TKTx 4 ExDD" & $+n$ \\
\hline & Precursor peptide contains "n" sequence motif \#9 "TxTxTx4Ex2DxD" & $+n$ \\
\hline$\sum_{\substack{++}}$ & SVM classifies as valid & +10 \\
\hline
\end{tabular}

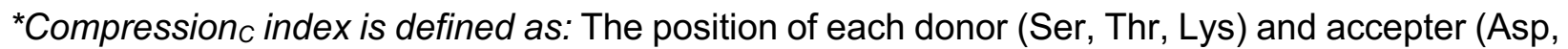
Glu) in the putative precursor peptide is divided by the length of the precursor peptide. The minimum, maximum, range, average, and standard deviation for donors and acceptors are then separated calculated. These values are then again averaged to give a single value for the C- 
terminal compression of acceptor and donor residues. About $97 \%$ of characterized and highconfidence predicted graspetide precursor peptides in the training set have accepter compression $_{\mathrm{C}}$ index greater than the donor compression $\mathrm{c}$ index.

Final positive cut-off score: 12 
Table S2. Graspetide module support vector machine parameters.

\begin{tabular}{|c|c|c|c|}
\hline Feature & Data type & Feature & Data type \\
\hline $\begin{array}{l}\text { Minimum distance from ATP-grasp } \\
\text { ligase (nt) }\end{array}$ & Integer & Precursor length & Integer \\
\hline Within 1100 nt of ATP-grasp ligase & Boolean & $\begin{array}{l}\text { Number of each amino acid in full- } \\
\text { length precursor }\end{array}$ & Integer \\
\hline Within 300 nt of ATP-grasp ligase & Boolean & $\begin{array}{l}\text { Number of each amino acid type } \\
\text { (aromatic, aliphatic, hydroxyl, basic, } \\
\text { acidic, donors, acceptors) in full- } \\
\text { length precursor }\end{array}$ & Integer \\
\hline $\begin{array}{l}\text { Further than } 2000 \mathrm{nt} \text { from ATP- } \\
\text { grasp ligase }\end{array}$ & Boolean & $\begin{array}{l}\% \text { of each amino acid in full-length } \\
\text { precursor }\end{array}$ & Float \\
\hline $\begin{array}{l}\text { Second half of precursor contains }> \\
8 \% \text { Asp residues }\end{array}$ & Boolean & $\begin{array}{l}\text { \% of each amino acid type } \\
\text { (aromatic, aliphatic, hydroxyl, basic, } \\
\text { acidic, donors, acceptors) in full- } \\
\text { length precursor }\end{array}$ & Float \\
\hline $\begin{array}{l}\text { Second half of precursor contains > } \\
7 \% \text { Thr residues }\end{array}$ & Boolean & $\begin{array}{l}\text { Number of each amino acid in the } \\
\text { first half of precursor }\end{array}$ & Integer \\
\hline $\begin{array}{l}\text { Second half of precursor contains }> \\
7 \% \text { Pro residues }\end{array}$ & Boolean & $\begin{array}{l}\text { Number of each amino acid type } \\
\text { (aromatic, aliphatic, hydroxyl, basic, } \\
\text { acidic, donors, acceptors) in first } \\
\text { half of precursor }\end{array}$ & Integer \\
\hline $\begin{array}{l}\text { Precursor (full-length) contains > } \\
7 \% \text { Lys residues }\end{array}$ & Boolean & $\begin{array}{l}\% \text { of each amino acid in the first } \\
\text { half of precursor }\end{array}$ & Float \\
\hline $\begin{array}{l}\text { Precursor contains }>0 \text { Cys } \\
\text { residues }\end{array}$ & Boolean & $\begin{array}{l}\text { \% of each amino acid type } \\
\text { (aromatic, aliphatic, hydroxyl, basic, } \\
\text { acidic, donors, acceptors) in the } \\
\text { first half of precursor }\end{array}$ & Float \\
\hline $\begin{array}{l}\text { Second half of precursor contains < } \\
3 \text { acceptor residues }(\text { Asp }+ \text { Glu) }\end{array}$ & Boolean & $\begin{array}{l}\text { Number of each amino acid in the } \\
\text { second half of precursor }\end{array}$ & Integer \\
\hline $\begin{array}{l}\text { Second half of precursor contains }< \\
3 \text { donor residues }(\text { Ser }+ \text { Thr }+ \text { Lys })\end{array}$ & Boolean & $\begin{array}{l}\text { Number of each amino acid type } \\
\text { (aromatic, aliphatic, hydroxyl, basic, } \\
\text { acidic, donors, acceptors) in second } \\
\text { half of precursor }\end{array}$ & Integer \\
\hline $\begin{array}{l}\text { Second half of precursor contains } \\
\text { more donor residues than acceptor } \\
\text { residues }\end{array}$ & Boolean & $\begin{array}{l}\% \text { of each amino acid in the second } \\
\text { half of precursor }\end{array}$ & Float \\
\hline $\begin{array}{l}\% \text { acceptor residues in the second } \\
\text { half of precursor }>\% \text { acceptor } \\
\text { residues in the first half of precursor }\end{array}$ & Boolean & $\begin{array}{l}\text { \% of each amino acid type } \\
\text { (aromatic, aliphatic, hydroxyl, basic, } \\
\text { acidic, donors, acceptors) in second } \\
\text { half of precursor }\end{array}$ & Float \\
\hline $\begin{array}{l}\% \text { acceptor residues }(\text { Asp }+ \text { Glu }) \text { in } \\
\text { the second half of precursor }>13 \%\end{array}$ & Boolean & $\begin{array}{l}\text { Calculated charge of first half of } \\
\text { precursor at } \mathrm{pH} 7\end{array}$ & Integer \\
\hline $\begin{array}{l}\% \text { donor residues }(\text { Ser }+ \text { Thr }+ \text { Lys }) \\
\text { in the second half of precursor }> \\
18 \%\end{array}$ & Boolean & $\begin{array}{l}\text { Calculated charge of second half of } \\
\text { precursor at } \mathrm{pH} 7\end{array}$ & Integer \\
\hline Precursor ends with Leu & Boolean & $\begin{array}{l}\text { Calculated precursor charge at } \mathrm{pH} \\
7\end{array}$ & Integer \\
\hline $\begin{array}{l}\text { Precursor ends with Asp or Val or } \\
\text { Ser }\end{array}$ & Boolean & $\begin{array}{l}\text { Absolute charge of first half of } \\
\text { precursor at } \mathrm{pH} 7\end{array}$ & Integer \\
\hline $\begin{array}{l}\text { Precursor and ATP-grasp ligase are } \\
\text { encoded in the same direction }\end{array}$ & Boolean & $\begin{array}{l}\text { Absolute charge of second half of } \\
\text { precursor at } \mathrm{pH} 7\end{array}$ & Integer \\
\hline $\begin{array}{l}\text { Gene cluster contains one of the } \\
\text { following: PF06472, PF03412, }\end{array}$ & Boolean & $\begin{array}{l}\text { Absolute value of precursor charge } \\
\text { at } \mathrm{pH} 7\end{array}$ & Integer \\
\hline
\end{tabular}




\begin{tabular}{|l|l|l|l|}
\hline $\begin{array}{l}\text { TIGR01846, TIGR03796, } \\
\text { TIGR03797, TIGR00954, } \\
\text { TIGR03375, TIGR02203 }\end{array}$ & Boolean & Presence of each of motifs 1-22 & Boolean \\
\hline $\begin{array}{l}\text { Gene cluster contains one of the } \\
\text { following: PF13302, PF00583, } \\
\text { PF13523, PF13673 }\end{array}$ & Boolean & Total motifs hit & Integer \\
\hline $\begin{array}{l}\text { Precursor hits PF12559 or } \\
\text { TIGR04186 }\end{array}$ & Boolean & $\begin{array}{l}\text { MEME/FIMO score of each of } \\
\text { motifs 1-22 }\end{array}$ & Float \\
\hline $\begin{array}{l}\text { Acceptor compressionc index }> \\
\text { Donor compressionc index }\end{array}$ & Boolean & Sum of MEME scores & Float \\
\hline $\begin{array}{l}\text { Calculated charge at pH7 of second } \\
\text { half of precursor < Calculated } \\
\text { charge at pH7 of first half of } \\
\text { precursor }\end{array}$ & Float & No motifs present & Boolean \\
\hline Acceptor compressionc index & Float & $\begin{array}{l}\text { Precursor ends with amino acid (for } \\
\text { each) }\end{array}$ & Boolean \\
\hline Donor compressionc index & \multicolumn{2}{|l}{} \\
\hline
\end{tabular}

Charge is calculated by the sum of residues predicted to have a charge at $\mathrm{pH}$ 7. Lys/Arg are taken to be +1 formal charge each, and Asp/Glu are considered to be -1 formal charge each. The net charge is defined as the sum of positive and negative charges. 
Figure S1. Graspetide module workflow. Steps common to all previously available RODEO scoring modules are grey. Steps unique to the graspetide-scoring RODEO module are red. Unlike precursor peptides of most other RiPP classes, graspetide precursor peptides are often longer and contain repeat sequences. To aid in the detection of such precursor peptides without compromising the computation time, a bioinformatics tool, RADAR ${ }^{11}$, was integrated into the graspetide-scoring RODEO module.

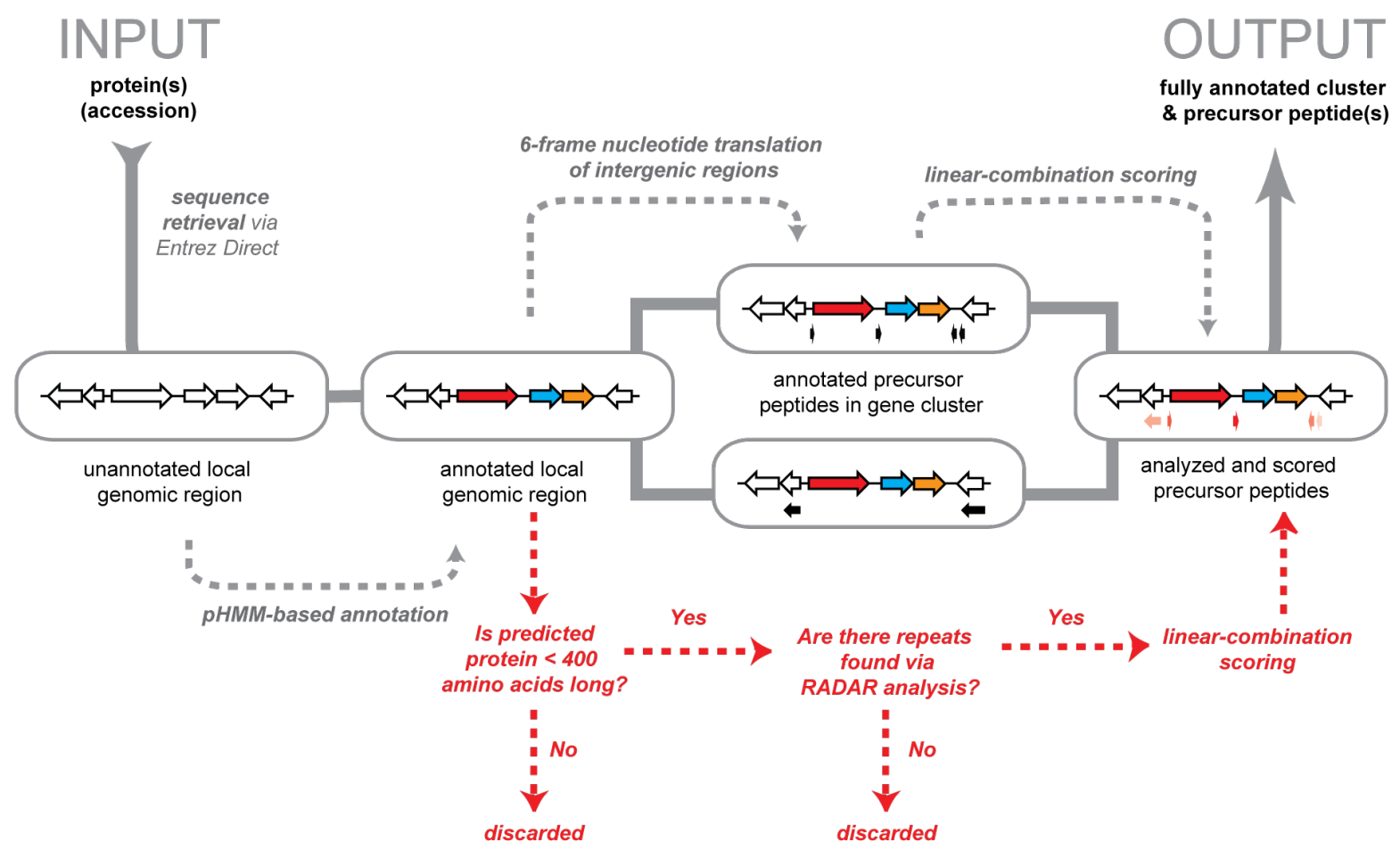


Figure S2. Validation of the graspetide scoring module. A. Scoring accuracy of the graspetide module on a randomly selected test set comprising groups 1-6 graspetides. Precision (true positives/observed positives) and recall (true positives/actual positives) represent specificity and sensitivity, respectively. B. Scoring distribution of peptides in the test set. A RODEO score of 12 gives the optimal separation of graspetides from hypothetical non-graspetide sequences.
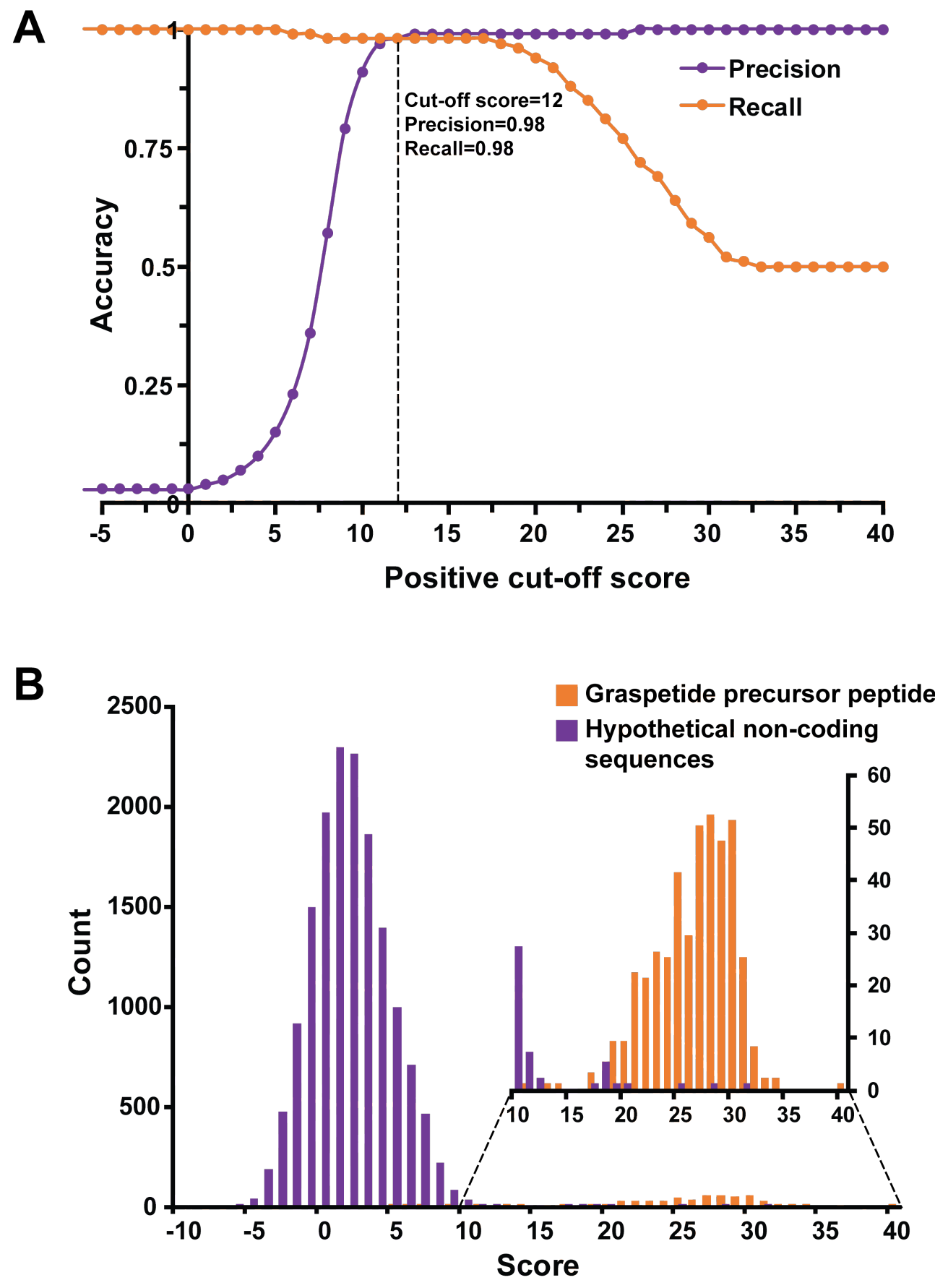
Figure S3. Sequence similarity network of graspetide precursor peptides. The SSN was generated using EFI-EST and visualized with Cytoscape. ${ }^{13,15}$ Nodes represent individual precursor peptide sequences $(n=4,356)$. Edges indicate relatedness at an alignment score of 9 (equivalent to a BLAST expectation value of $10^{-9}$ ). Group numbers are indicated next to clusters. Example of a characterized member, if available, is mentioned in parentheses below group number. Previously reported graspetide groups (1-12) have white nodes. Groups 13-24 have colored nodes with group 16 (thatisin, first reported in this work) colored yellow. "Others", which included very small groups or singletons, have gray nodes.
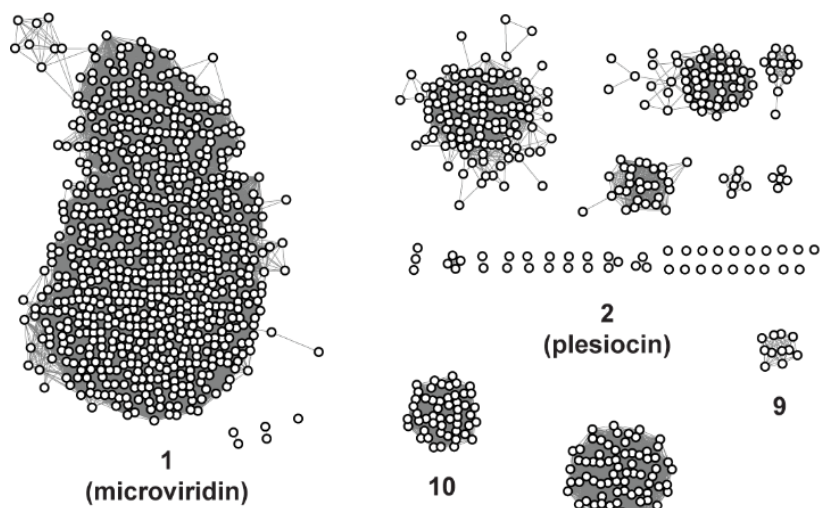

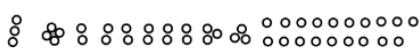
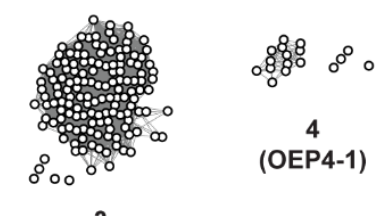

$\stackrel{3}{\text { (thuringinin) }}$

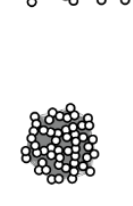
2
(plesiocin)

10
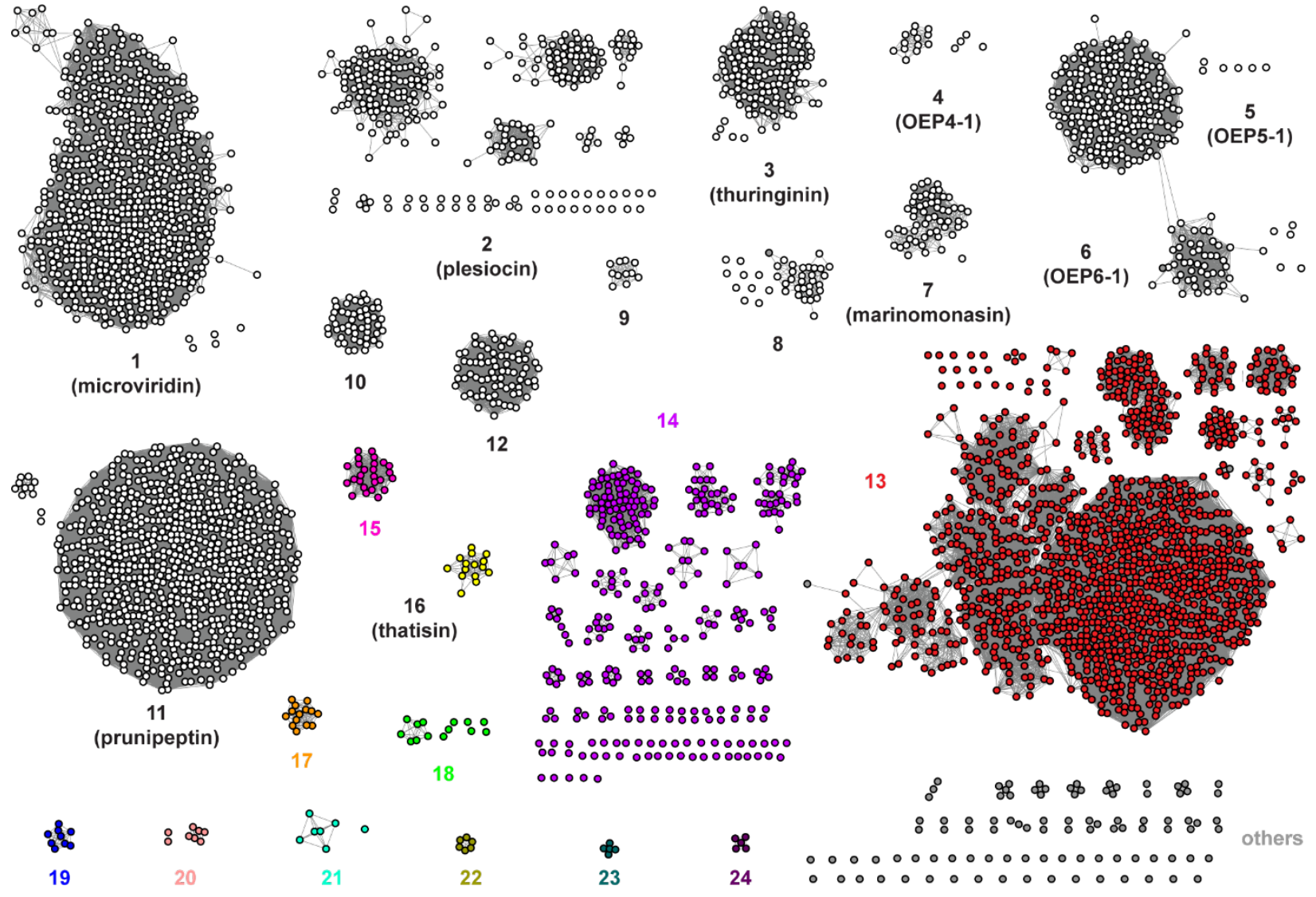

8
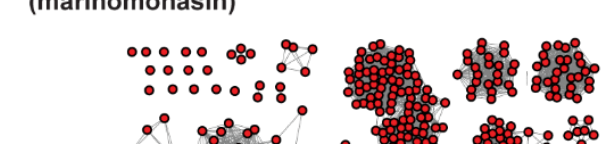

12

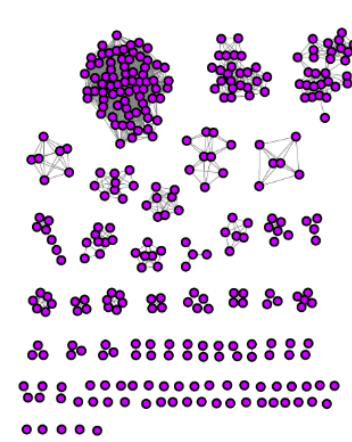

.000

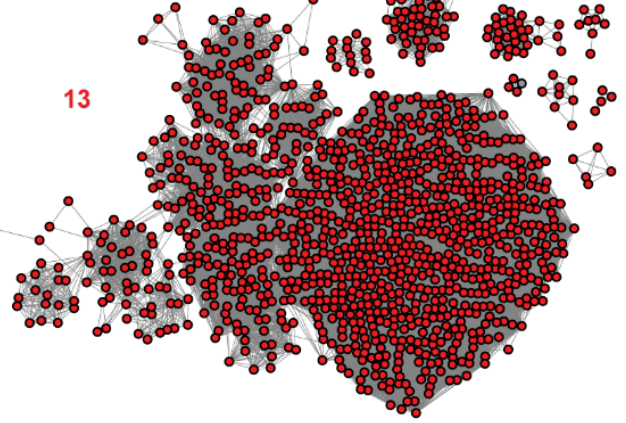

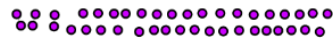

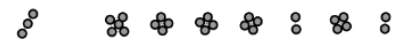

: ஃ : others 
Table S3. Distribution of graspetide biosynthetic gene clusters. The number of identified graspetide BGCs is given per prokaryotic phylum. A representative member from each characterized group is red. Thatisin (group 16) is elucidated in the current study. Others ${ }^{*}$ indicates an origin of uncultured, unclassified, or a candidate division.

\begin{tabular}{|c|c|c|c|c|c|c|c|c|c|c|c|c|c|c|c|c|c|}
\hline Group ID\# & $\begin{array}{c}\# \\
\text { BGCs }\end{array}$ & 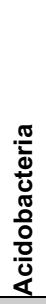 & 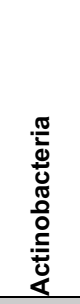 & 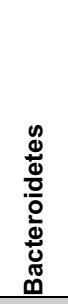 & $\begin{array}{l}\bar{x} \\
\frac{0}{4} \\
\frac{0}{0} \\
\frac{0}{\bar{c}}\end{array}$ & 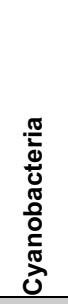 & 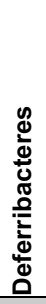 & 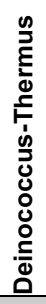 & 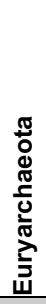 & 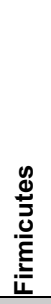 & 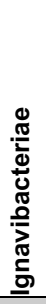 & 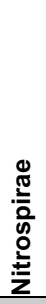 & 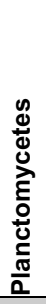 & 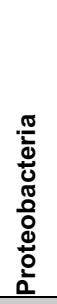 & 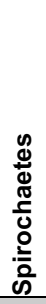 & 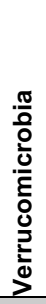 & 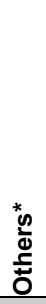 \\
\hline 1 (Microviridin K) & 451 & 2 & 0 & 210 & 0 & 197 & 0 & 0 & 0 & 0 & 0 & 0 & 1 & 40 & 0 & 0 & 1 \\
\hline 2 (Plesiocin) & 260 & 2 & 3 & 1 & 0 & 43 & 0 & 0 & 4 & 18 & 0 & 0 & 14 & 172 & 0 & 0 & 3 \\
\hline 3 (Thuringinin) & 143 & 0 & 0 & 0 & 0 & 0 & 0 & 0 & 0 & 37 & 0 & 0 & 0 & 105 & 0 & 0 & 1 \\
\hline 4 (OEP4-1) & 15 & 0 & 0 & 2 & 0 & 0 & 0 & 0 & 0 & 8 & 0 & 0 & 0 & 4 & 0 & 0 & 1 \\
\hline 5 (OEP5-1) & 237 & 0 & 0 & 20 & 1 & 0 & 1 & 0 & 0 & 13 & 0 & 0 & 0 & 200 & 2 & 0 & 0 \\
\hline 6 (OEP6-1) & 47 & 0 & 0 & 38 & 0 & 0 & 0 & 0 & 0 & 0 & 0 & 0 & 3 & 1 & 4 & 0 & 1 \\
\hline 7 (Marinomonasin) & 56 & 0 & 0 & 3 & 0 & 0 & 0 & 0 & 2 & 14 & 1 & 0 & 0 & 36 & 0 & 0 & 0 \\
\hline 8 & 32 & 0 & 0 & 0 & 0 & 0 & 0 & 0 & 0 & 0 & 0 & 0 & 0 & 32 & 0 & 0 & 0 \\
\hline 9 & 11 & 0 & 11 & 0 & 0 & 0 & 0 & 0 & 0 & 0 & 0 & 0 & 0 & 0 & 0 & 0 & 0 \\
\hline 10 & 51 & 0 & 0 & 2 & 0 & 0 & 0 & 0 & 0 & 1 & 0 & 0 & 0 & 48 & 0 & 0 & 0 \\
\hline 11 (Prunipeptin) & 777 & 0 & 776 & 0 & 0 & 0 & 0 & 0 & 0 & 0 & 0 & 0 & 0 & 1 & 0 & 0 & 0 \\
\hline 12 & 74 & 0 & 74 & 0 & 0 & 0 & 0 & 0 & 0 & 0 & 0 & 0 & 0 & 0 & 0 & 0 & 0 \\
\hline 13 & 1,326 & 1 & 1,308 & 0 & 17 & 0 & 0 & 0 & 0 & 0 & 0 & 0 & 0 & 0 & 0 & 0 & 0 \\
\hline 14 & 245 & 0 & 0 & 244 & 0 & 0 & 0 & 0 & 0 & 0 & 0 & 0 & 0 & 1 & 0 & 0 & 0 \\
\hline 15 & 26 & 0 & 0 & 0 & 0 & 0 & 0 & 0 & 0 & 0 & 0 & 0 & 0 & 26 & 0 & 0 & 0 \\
\hline 16 (Thatisin) & 14 & 0 & 0 & 0 & 0 & 0 & 0 & 0 & 0 & 0 & 0 & 0 & 0 & 14 & 0 & 0 & 0 \\
\hline 17 & 13 & 0 & 0 & 0 & 0 & 13 & 0 & 0 & 0 & 0 & 0 & 0 & 0 & 0 & 0 & 0 & 0 \\
\hline 18 & 11 & 0 & 0 & 0 & 0 & 0 & 0 & 0 & 0 & 0 & 0 & 0 & 0 & 11 & 0 & 0 & 0 \\
\hline 19 & 9 & 0 & 0 & 0 & 0 & 0 & 0 & 0 & 0 & 0 & 0 & 0 & 0 & 9 & 0 & 0 & 0 \\
\hline 20 & 8 & 0 & 0 & 0 & 0 & 0 & 0 & 0 & 0 & 0 & 0 & 0 & 0 & 8 & 0 & 0 & 0 \\
\hline 21 & 7 & 0 & 6 & 0 & 0 & 0 & 0 & 0 & 0 & 0 & 0 & 0 & 0 & 1 & 0 & 0 & 0 \\
\hline 22 & 6 & 1 & 0 & 0 & 0 & 0 & 0 & 0 & 0 & 0 & 0 & 0 & 0 & 5 & 0 & 0 & 0 \\
\hline 23 & 5 & 0 & 0 & 0 & 0 & 0 & 0 & 0 & 0 & 0 & 0 & 0 & 0 & 5 & 0 & 0 & 0 \\
\hline 24 & 5 & 0 & 5 & 0 & 0 & 0 & 0 & 0 & 0 & 0 & 0 & 0 & 0 & 0 & 0 & 0 & 0 \\
\hline Ungrouped & 94 & 4 & 22 & 1 & 5 & 5 & 0 & 1 & 2 & 3 & 0 & 3 & 0 & 34 & 0 & 1 & 13 \\
\hline Total & 3,923 & 10 & 2,205 & 521 & 23 & 258 & 1 & 1 & 8 & 94 & 1 & 3 & 18 & 753 & 6 & 1 & 20 \\
\hline
\end{tabular}


Figure S4. Representative graspetide biosynthetic gene clusters from new groups. One representative example is provided for each new graspetide group. Similar examples for groups 15 and 16 are in Figures 3 and S9. NCBI accession identifiers are provided for the graspetide synthetase. PCAT, Peptidase-containing ATP-binding transporter; DUF, domain of unknown function.

\section{Group 13}

Group 14

Group 19

\section{Group 17}

Group 18

Group 20

Group 21

Group 22

Group 23

Group 24

\section{Actinomadura flavalba DSM 45200 (WP_026313804.1)}

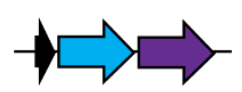

Sphingobacterium detergens (WP_120260689.1)

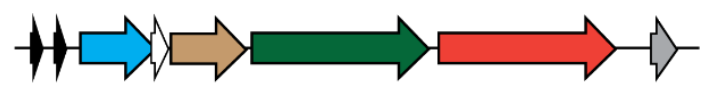

Limnothrix sp. PR1529 (WP_099533029.1)

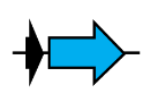

Caulobacter flavus (WP_101714393.1)

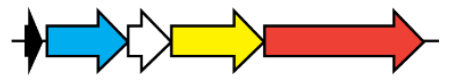

Lysobacter enzymogenes (WP_123647320.1)

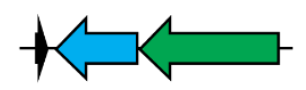

Stenotrophomonas maltophilia (PAM71714.1)

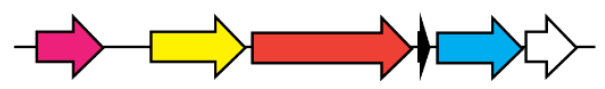

Micromonospora rifamycinica (WP_067309147.1)

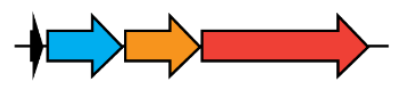

Burkholderia ubonensis (WP_157649645.1)

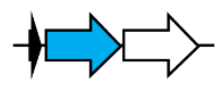

Xanthomonas melonis (WP_104587709.1)

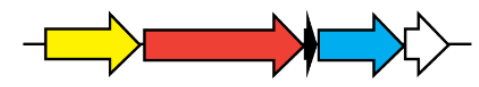

Rarobacter faecitabidus (TQL58573.1)

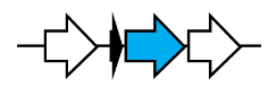

Precursor peptide

Graspetide synthetase

Methyltransferase

Unknown

SPASM domain-containing protein

Outer membrane protein

PCAT

DUF2200

HlyD

Transporter

Regulator

Peptidyl prolyl isomerase

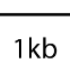

b 
Figure S5. Precursor peptide diversity of Grasp-with-Methyltransferase group graspetides. The Grasp-with-Methyltransferase (GwM) group (group 13; InterPro: IPR026449 and IPR026448) $)^{7,29,30}$ was divided into five subgroups based on conserved motifs in the presumed leader peptide region (orange). The microviridin-like putative recognition motif in subgroup 1 is boxed. Subgroups 3 and 4 are further subdivided into a-h and a-l, respectively, based on conserved putative macrolactone/ macrolactam acceptors and donors in the core region shown in blue and green, respectively. GwM subgroup numbers are indicated on the left of each sequence logo. ${ }^{20}$ Poorly conserved regions have been removed for clarity.

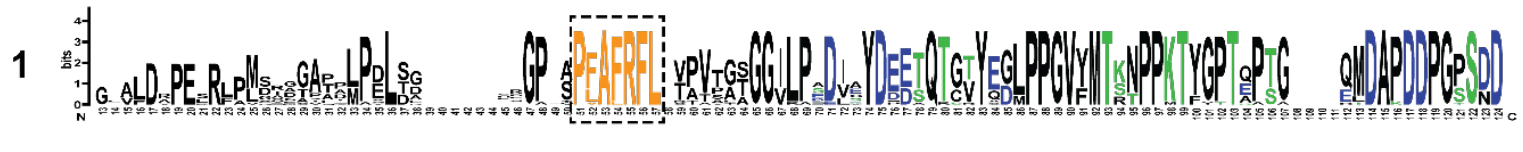

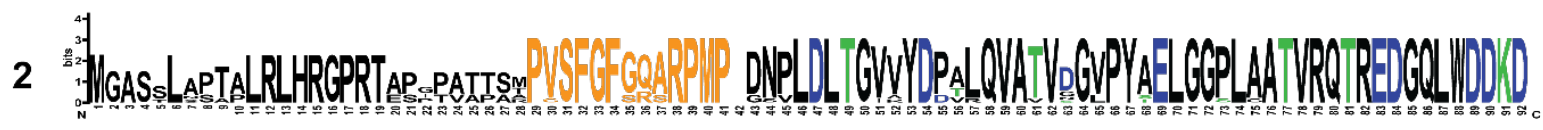
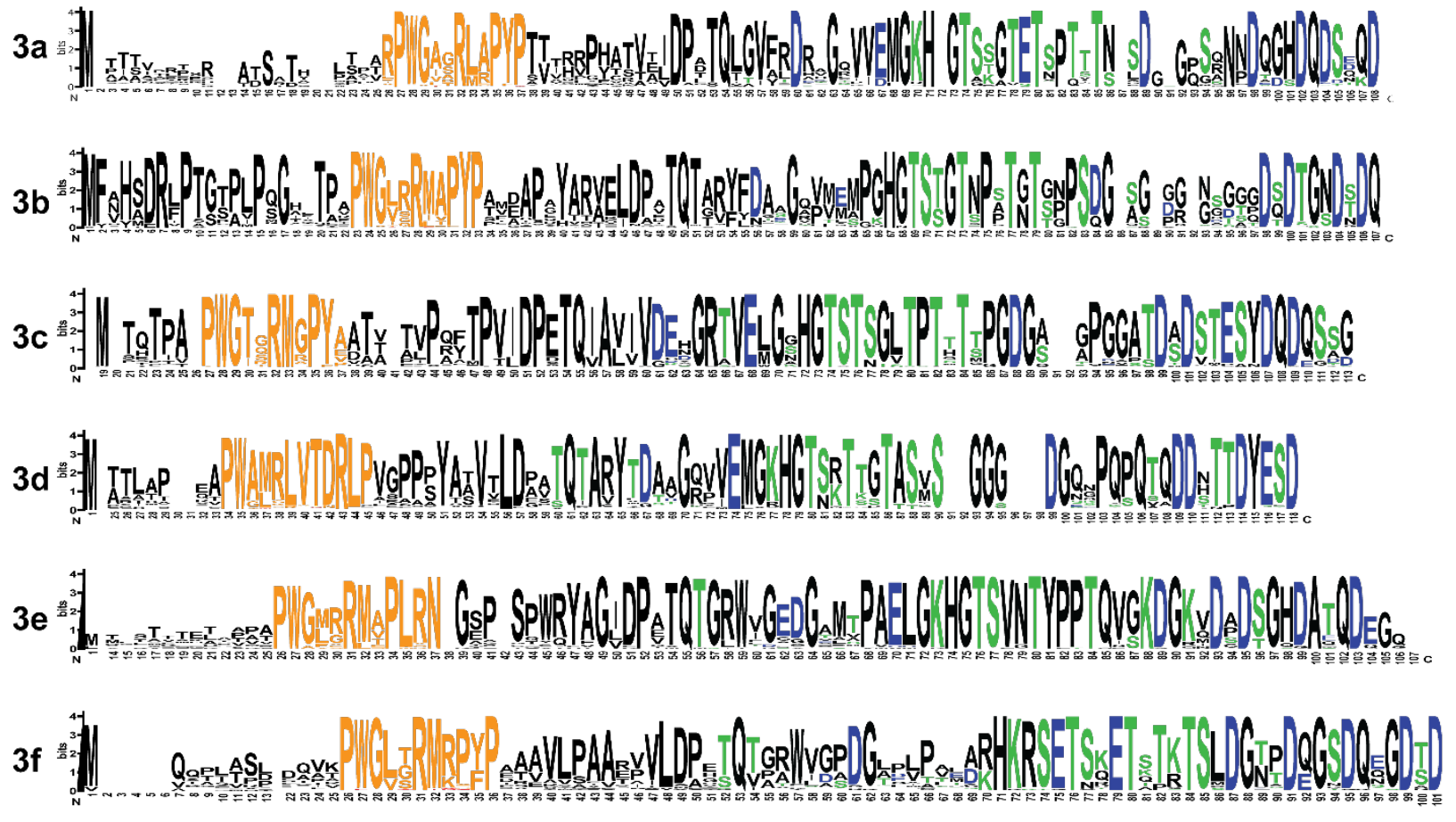

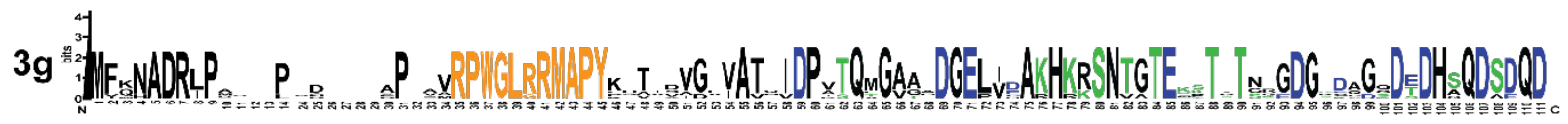

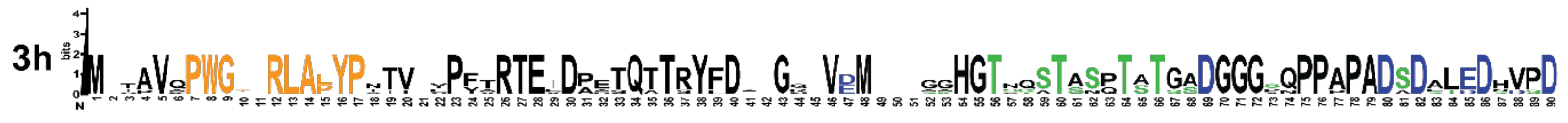

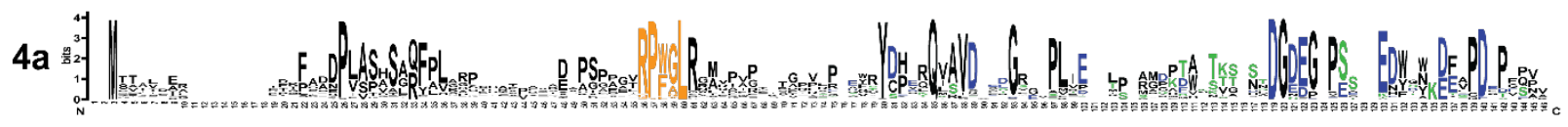




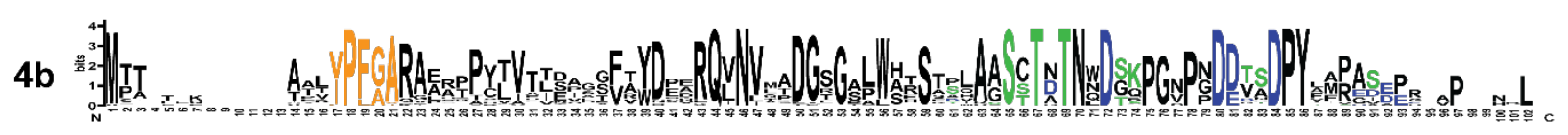

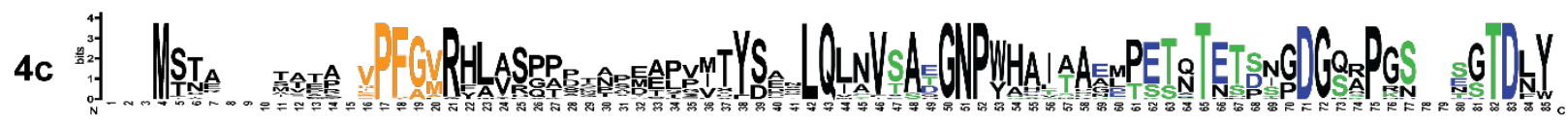

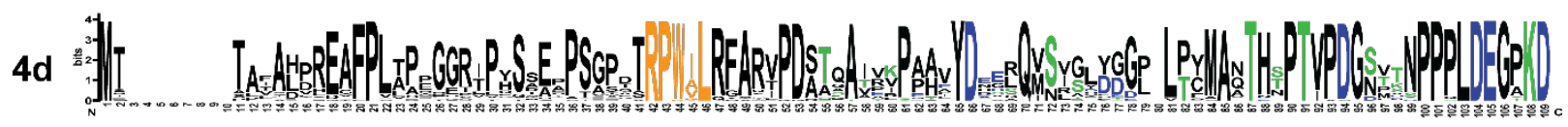

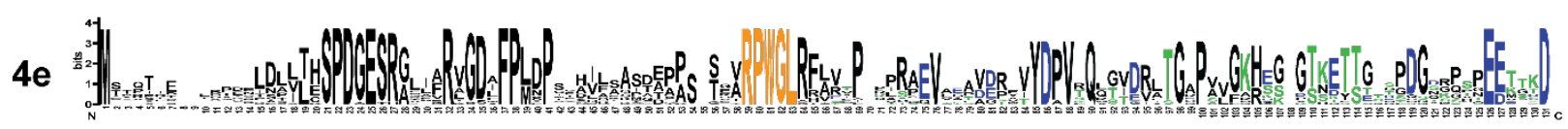

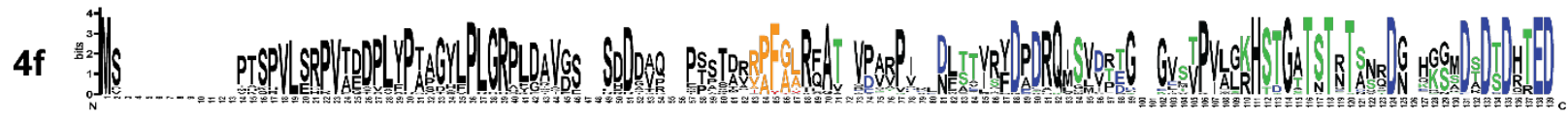

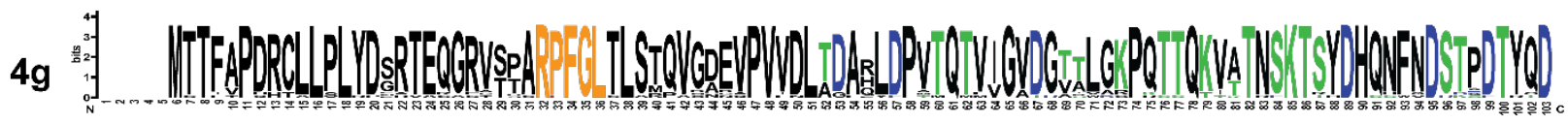

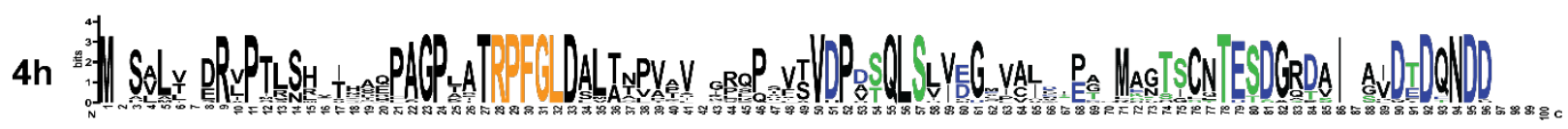

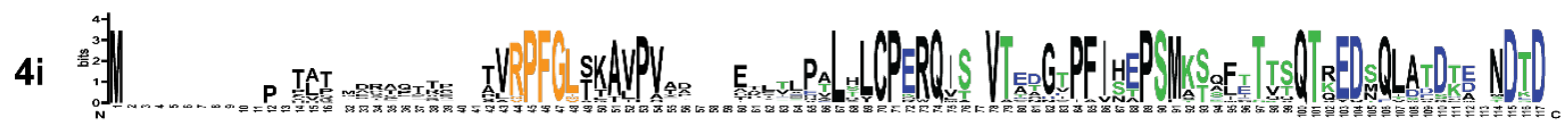

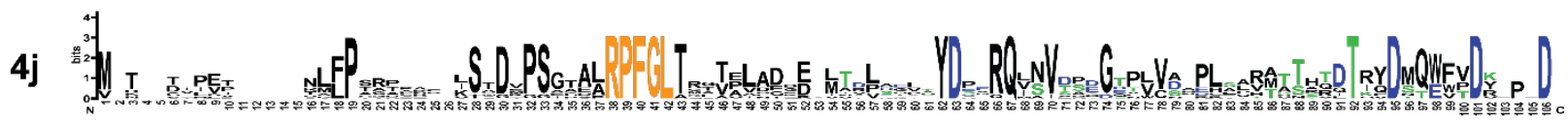

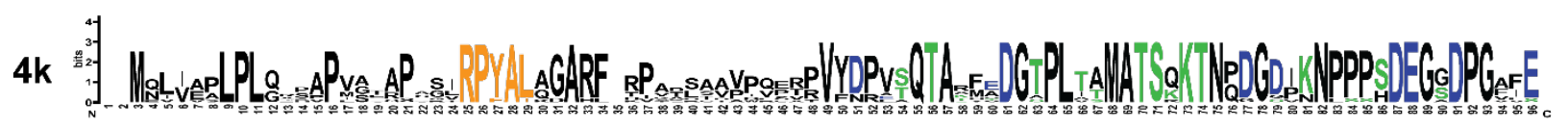

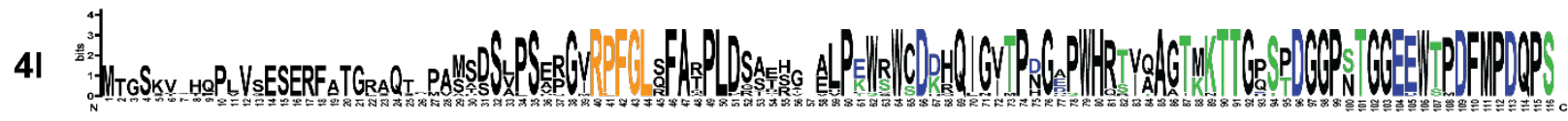

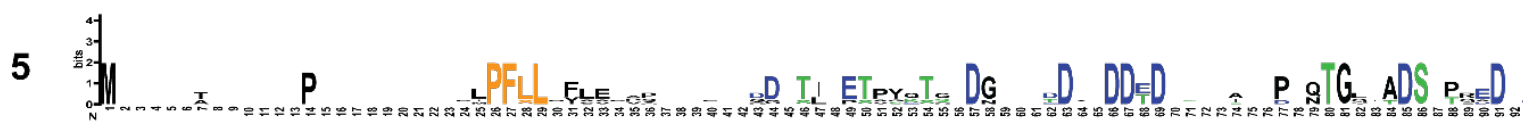


Figure S6. Sequence alignment of SPASM domain-containing proteins. Representative SPASM domain-containing protein sequences from Grasp-with-SPASM (GwS) group were aligned with representative, characterized radical SAM enzymes harboring a SPASM domain using MUSCLE. ${ }^{31}$ Cys residues coordinating the SAM-binding [4Fe-4S]cluster, SPASM-domain [Fe-S] clusters, and an additional conserved Cys in GwS of unknown significance are yellow, cyan, and red, respectively. PqqE, pyrroloquinoline quinone maturase; anSME, anaerobic sulfatase maturation enzyme.

WP_004184158.1। PqqE WP_003476924.1/anSME WP $049036919.1 \mid \mathrm{GWS}$ WP_076449952.1/GWS WP_002984443.1/GWS WP_083252239.1।GWS WP_160137504.1।GWS

WP_004184158.1|PqqE WP_003476924.1 I anSME WP 049036919.1 |GWS WP 076449952.1 |GwS WP 002984443.1 । GwS WP 083252239.1 । GWS WP 160137504.1 |GwS

WP_004184158.1। PqqE WP 003476924.1 IanSME WP_049036919.1।GWS WP_076449952.1।GWS WP_002984443.1।GWS WP_083252239.1/GWS WP_160137504.1/GWS

WP_004184158.1। PqqE WP 003476924.1 IanSME WP_049036919.1। GwS WP_076449952.1।GWS WP_002984443.1। GWS WP 083252239.1 | GWS WP_160137504.1/GWS

WP 004184158.1 | PqqE WP 003476924.1 I anSME WP_049036919.1। GwS WP 076449952.1 । GWS WP 002984443.1 | GwS WP 083252239.1 | GWS WP_160137504.1/GWS

WP_004184158.1। PqqE WP_003476924.1 I anSME WP 049036919.1 | GwS WP_076449952.1।GWS

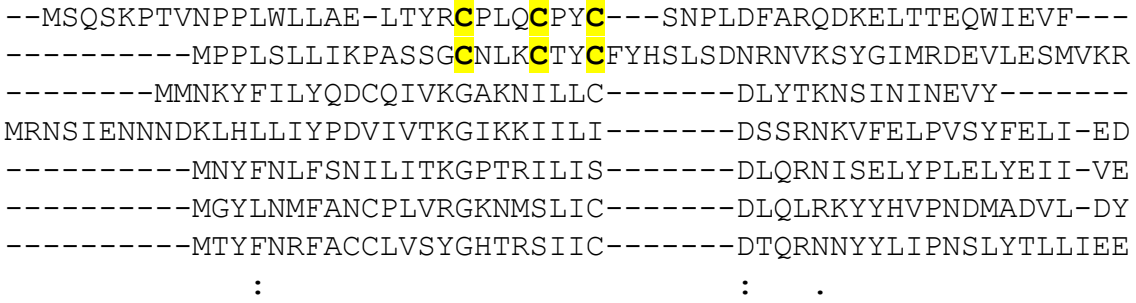

RQARAMGSVQLGFSGGEPLT--RKDLPELIRAARDLGF-----YTNLITSG--IGLTESK VLNEADGHCSFAFQGGEP ILAGLEFFERLMELQRKHNYKNLKIYNSLQTNGTLIDESWAK --SYFEDDLSILYGDKS------KEIIDFL-IEEKFGYIS---VEKSTHR---KSLQWGS FKKYTIHDIKGIYDYEN-----LEMFINFI-ITNDLGFFTDN--KDSFPA---ISTQYLS LKSHSIETILKDYDQESRSI--VHEYIDLL-LEKEYGFITENDWDRNFPS---LSFEHHE LKSHSVEECYDAYGYGNKGV--IKSYVDFV-LKNDMGFMDTRIMDELVP----LPLQWDA FPNFSIEEIYDSYGKENEKY--LKDYINFL-LVNEIGFIDSKIIEELIP----LNLNYKV

$$
\text { : } \quad:: \quad .:
$$

LDAFSEAGLDHIQISFQASDEVLNAALAGNKKAFQQKLAMAKAVKARDYPMVLNFVLHRH FLSENKFLVGLSMDGPKEIHNLNRKDCCGLDTFSKVERAAELFKKYKVEFNILC-VVTSN SKLINEIIIEHSRNEGFSIENVY-KKLEFIG-AEFIQIRFID-YSFRKLKKVLS-LLSSS PEQINNSI IEIDRREQLLNIERWLVDLDNLG-CKFYELRFYLNIDPSTISGIFR-EFKFN PSTITNLFIEMADIGVLKKIYTSVENLG----IKHLVIYSLNPLTSKDFIEIDT-IFKNS YADITNVIVELSPE--MANDSCFIEELVNLN-LEAIEIRCYEAISSEKLTQFLN-HFSKS AEPISNIIIDVSKESFFLEDSVHIAQFIDTNRVKHLQIRCYDAIDLNVFLKFLN-NLSRT : : :

NIDQLDKI IELC IELEADDV-ELATC--------QFYGWAFLNRE-----GLLPTREQIA TARHVNKIYRYFKEKDFKFL-QFINCLDPLYEEKGKYNYSLKPQDYT---KFLKNLFDLW NIRTIEILVPYMDEDKNSAL-ISYVEKNPRVQI LYFYNSPFNKSI------NKPFYFNVI SLNGINLYLQFSADYRVASY-AKLLNSFPFIKNIFILQSSRSKIVNATENKKLNNEQKI I VLSGIEVFSPFHQKINLSFV-QALQKKTVRLYSLIFYNCSKP PFK-----AKNEYKFSLH IVGTIRIFLKWNNWCTEKLF-KNLIENNLRINS IVVHSSPQEKTI-----KLLKNSVSIL TLKGIEIILPYEIKLGNRLLNKSMIKSNDKINKLIYFGCDKYKQE-----KYMNRI--IT

RAEQVVADYRQKMAASGNLTNLLFVTPDYYEERPKGCMGGWGSIFLSVTPEGTALPCHSA YE-----DFLNGNRVS IR-YFDGLLETILLGKSSSCGMNGTCTCQFVVESDGSVYPCDFY YYERNLTDEKLCGI INTD-YFLIDIKNFSKSKNVNNCLKD----KLFISRNGTIKNCPSFSTDVL-SRKNCGKVSSDMFFPREINQISENLCFNSCLFK----KLSIDIDGNIKNCPSFLKDDL-KISACGKVELK-YFNTNIPKVIEAVNHNSCLYK----KMGIDKNGNLKNCPLYKKEVIASCLQCGVIDPG-YFRTNIDLFTESQKHNTCLNR----KLSINKDGY IKNCPSYVEDKI INQKSCGLISSK-YFNSNLETYTLSNSHNSCLYK----KISIDSEGNIRNCPS-

RQLPVAFPSVLEQSLES IWYDSFGFNRYRGYDW-MPEPCRSCDEKEKDFGGCR--CQAFM VLDKWRLGNIQDMTMKEL-FETNKNHEF IKSSFKVHEECKKCKWFKLCKGGCR---RCRD --LNLEIGHINDILIEDL-YTVIEADPIRNITKDSIEICKDCEYRYICT-DCR---AYLE --MAKSYGNIKNESIDSV-IKRIDFKEYWSVTKDDIKICQDCEYRYICT-DCR---AYTE 
WP $002984443.1 \mid \mathrm{GWS}$ WP 083252239.1 | GWS WP 160137504.1 |GwS

WP_004184158.1। PqqE WP 003476924.1 I anSME WP_049036919.1।GwS WP 076449952.1 । GWS WP $002984443.1 \mid \mathrm{GWS}$ WP $083252239.1 \mid \mathrm{GWS}$ WP_160137504.1। GWS
--MNESFGNINSQSLEET-LNQSGFKKYWNLTKDYIETCKDCEFRYVCT-DCR---AYTE --MPESFGHISQTRLADV-LANPEFKKNWLLNKDRIAVCKDCEFRHVCT-DCR---AYVE --MPQSFGNIKDITLDEA-VSHPDFKKYWNLTKDSIEVCKDCEFRYICT-DCR---AYTE : $: \quad$. $\star \ldots *$.

LTGSADN-----ADPVCSKSPHHHKILEARREAACSDIKVSQLQFRNRTRSQLIYQTRDL SKEDSD---LELNY-YC------QSYKEFFEYAFPRLINVANNIK--------------KPSNPL-----SKPLKCGYDPYKGIWNDWTKNPLKQTTIKYYEIEIN-----------RNHFNEDNLDISKPLKCGYNPYTSKWEQWSTNPLKEQAIKYYGMQELVKNK-------NAEKNKEGLNISKPLKCGYNPYTAAWEDWSKNPLKQEIFHSLELR------------QPQDRY-----SKPLKCGYDPYANVWEDWATSPLKQEAIAYYGLEK------------RTHTNSEGLDTSKPLKCGYNPYTSEWEEWSTNPLKEKA INYYGMQELVKEK--------- 
Figure S7. Core peptide diversity among Grasp-with-SPASM group graspetides. The Grasp-

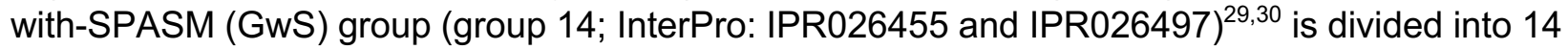
subgroups based on conserved acceptor (blue) and donor (green) residues in the core region. Sequence logos were generated for aligned full-length precursor peptides. However, only the Cterminal portion following the conserved Gly-Gly motif is shown, except for subgroup 5, whose Cterminal half is shown since these sequences lack a conserved Gly-Gly motif. The numbers below the logo represent the GwS subgroup.

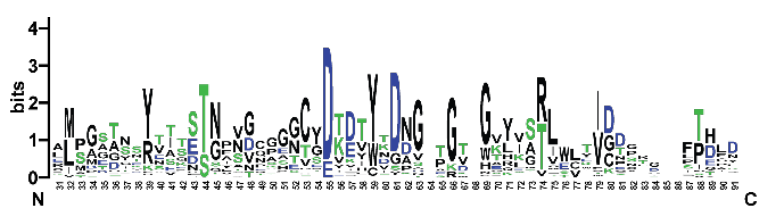

1

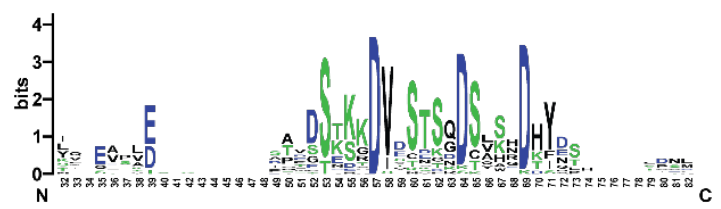

2

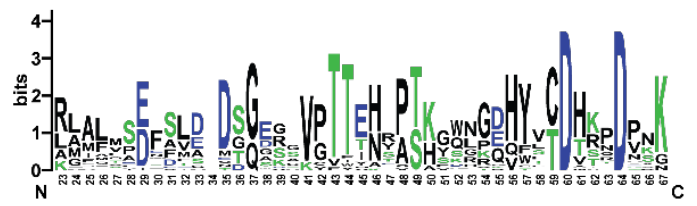

3

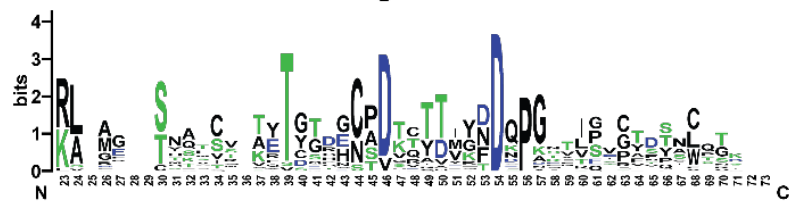

4

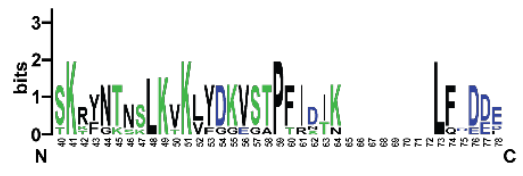

5

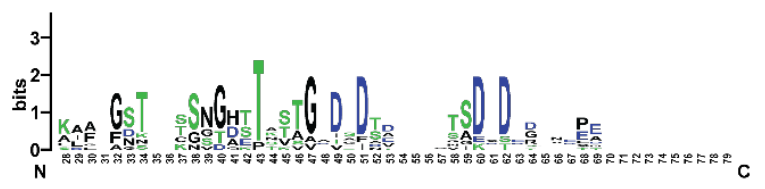

6

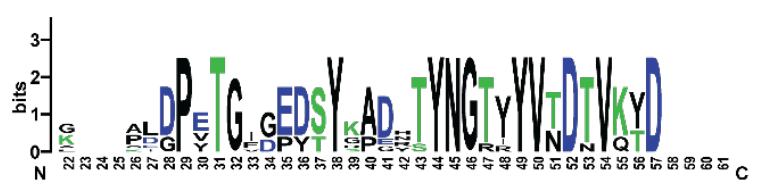

7

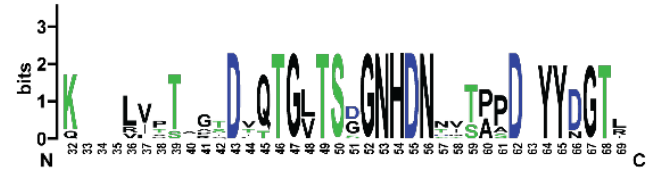

8

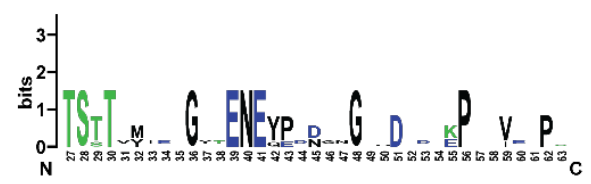

9

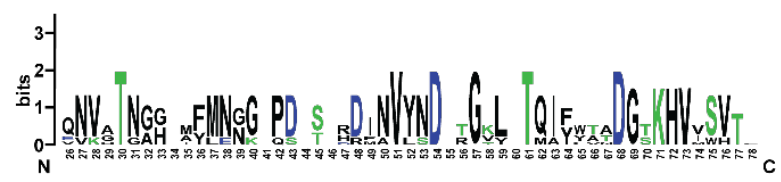

10

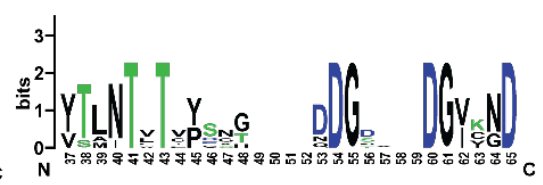

11

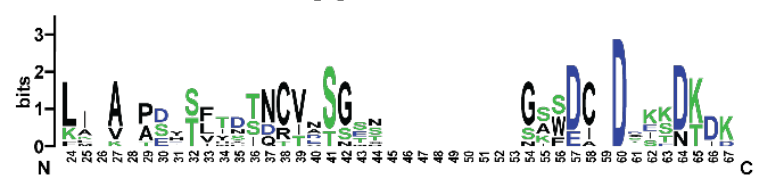

12

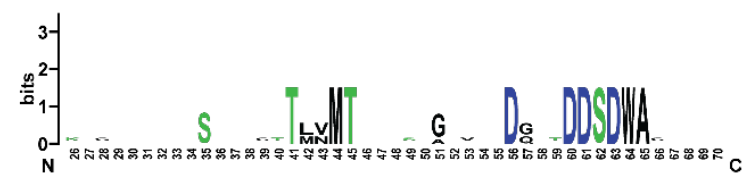

13

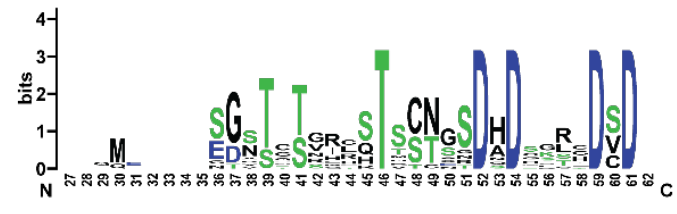

14 
Figure S8. Leader peptide sequences of Grasp-with-SPASM group graspetides. Sequence logos for the N-terminal leader region of GwS subgroup precursor peptides are shown. Lys and Leu residues in the leader region are in red. The GwS subgroup is noted below the logo.

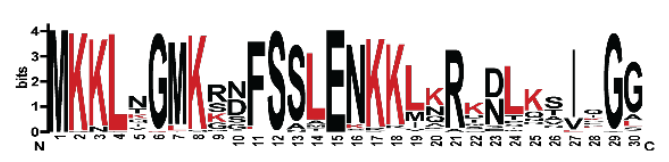

1

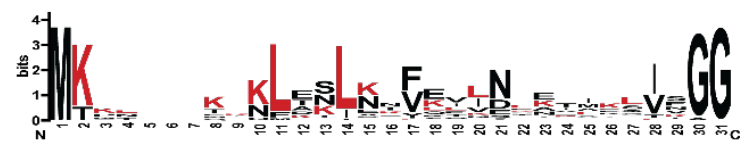

2

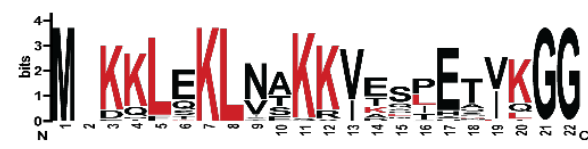

3

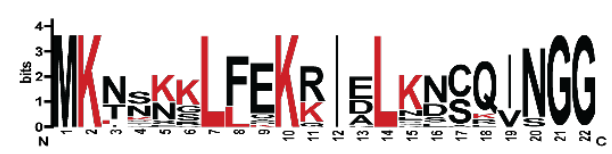

4

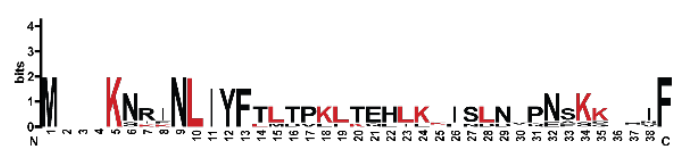

5

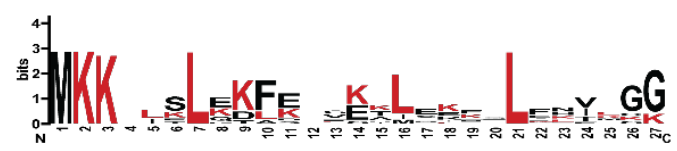

6

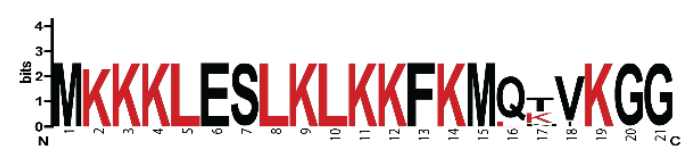

7

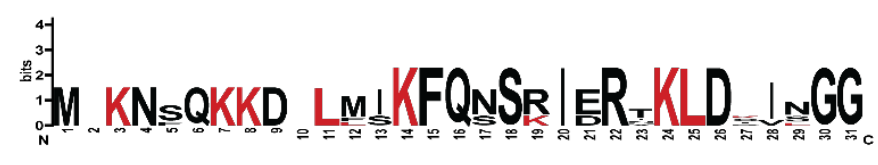

8

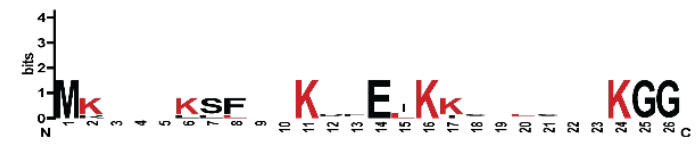

9

: MWLESISESKF:

10
11

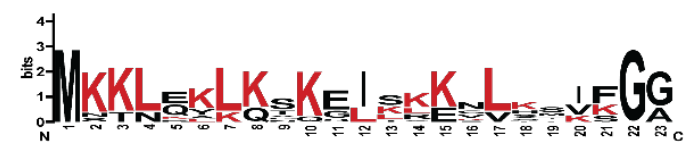

12

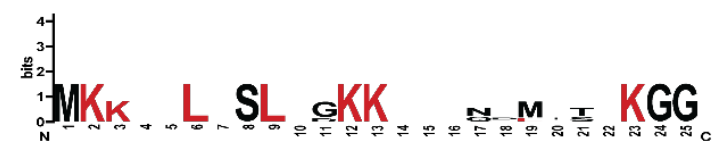

13

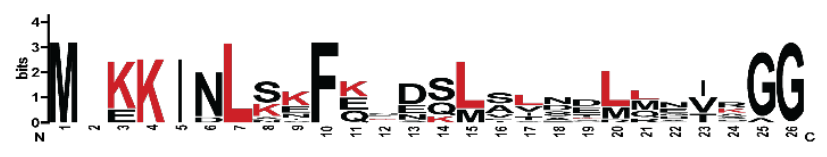

14 
Figure S9. Sequence analysis of new graspetide groups. Sequence logos for precursor peptides from groups 17-23 were generated using Weblogo. ${ }^{20}$ Potential acceptor and donor residues for macrolactone/macrolactam formation in the presumed core region are blue and green, respectively.

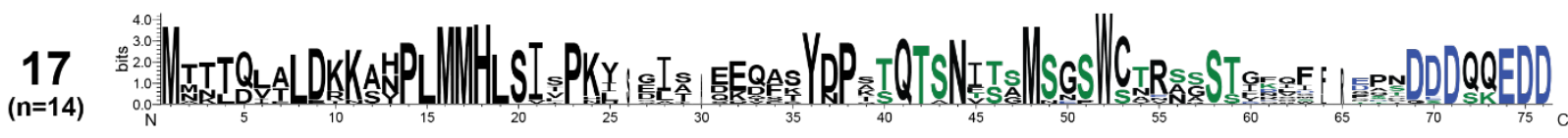

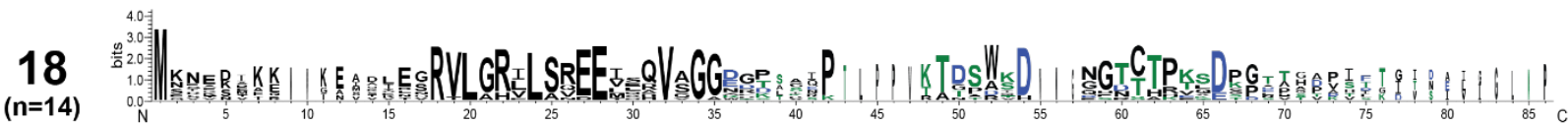

19.

20

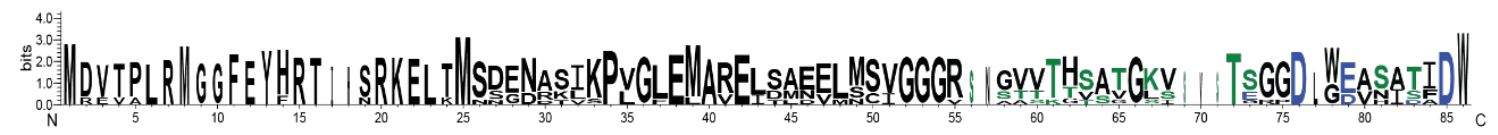

21 2 a :

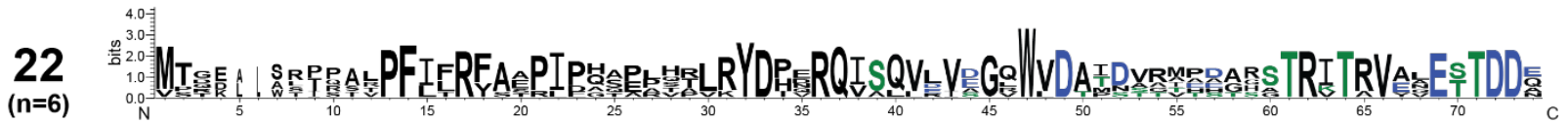

23. 2.

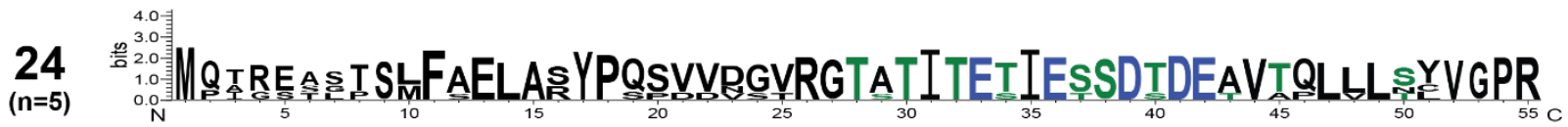


Figure S10. Representative biosynthetic gene cluster for group 15 graspetides. One representative group 15 graspetide BGC (Graspetide synthetase: WP_121758878.1) with repeats found in the core region of the precursor peptide is shown. Conserved macrolactone/macrolactam acceptor and donor residues are in blue and green, respectively.

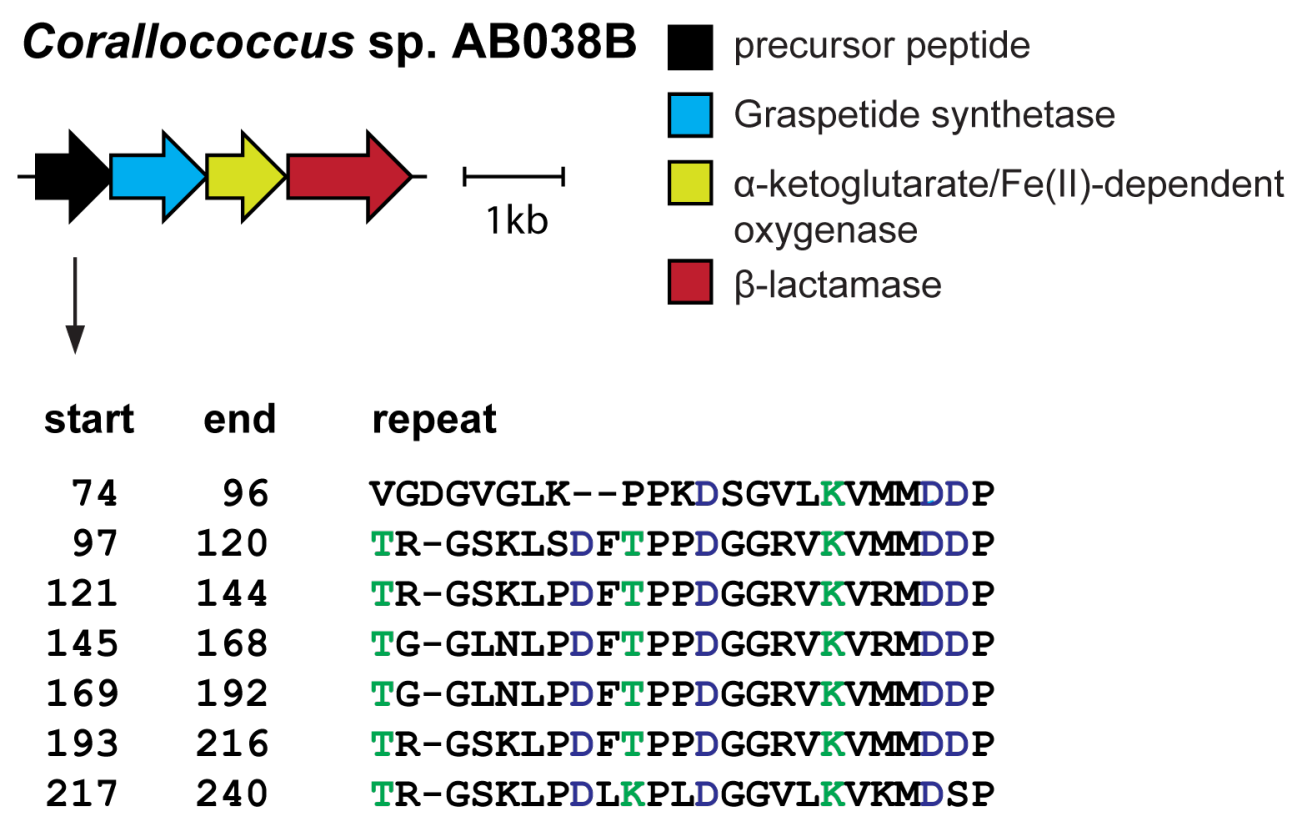


Figure S11. Hybrid RiPP BGCs featuring a graspetide synthetase. A. RiPP BGC from Actinomadura roseirufa (Graspetide synthetase: WP_131735415.1) featuring YcaO, TfuA, and ThiS homologs involved in thioamide formation. ${ }^{32,33}$ The putative core is bold, and the putative macrolactone/ macrolactam acceptor and donor residues are blue and green, respectively. PCAT, peptidase-containing ATP-binding transporter. B. RiPP BGC from Nitrospira sp. (Graspetide synthetase: TKB91036.1) featuring genes typically associated with lasso peptide biosynthesis. This BGC, however, lacks the lasso cyclase that installs the class-defining macrolactam. The BGC was annotated using RRE-Finder ${ }^{6}$ and RODEO. The putative core regions are in bold, and putative macrolactone/ macrolactam acceptor and donor residues are blue and green. The "YxxP" recognition sequence that is predicted to bind lasso peptide-like RiPP Recognition Element (RRE) domains is shown in red. ${ }^{34}$ Three out of 5 precursor peptides score below the validity threshold score owing to divergence from known graspetides.

\section{A Actinomadura roseirufa}

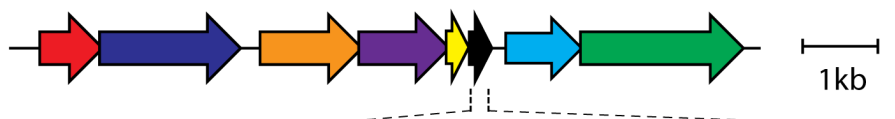

METAPQVMAGHLLSEVEPIHGTLDVLADRDAKQQLDRLVDYLMDPEPTEAFKTVGMSTQTHTGTDTGHSSTNTVPDADSDSEWQWDDE
Thioesterase domain
This
Amino acid adenylation domain
TfuA
Precursor peptide
YcaO
$\square$ Graspetide synthetase
$\square$ PCAT

\section{B Nitrospira sp.}

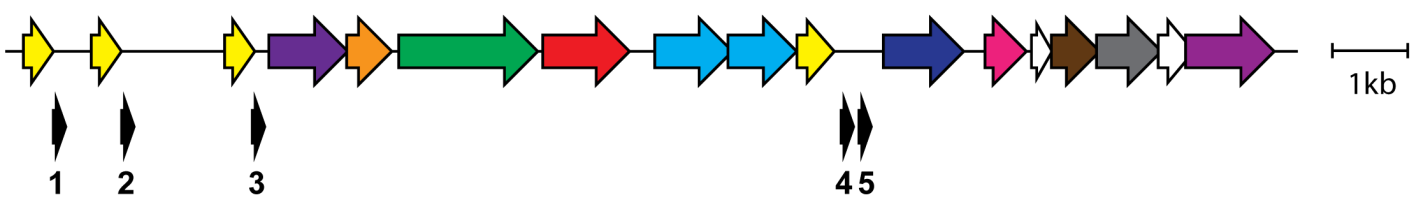

$\begin{array}{lcl}\mathbf{1} & \text { MNTAKKESYVQPALERHELLRDITAQSSGNNNCNGSEKRCED } & \\ \mathbf{2} & \text { MEQKKEQYVQPVVVKHELLRDITAGQSGYVRECEK } & \\ \mathbf{3} & \text { MNTAKKEPYVQPALEHELLRDITAQSSGYNYCNGSEKKSCEN } & \\ \mathbf{4} & \text { MEIQQKESYTEPVLVAHEMLRDITGGTSGVKTSDKTGKESDGTSF } & \\ \mathbf{5} & \text { MEQQKKESYAEPVLIVHELLRDITGQSSGGGCQKALDNTCVD } & \\ \square \text { RRE } & \square \text { Nucleotidyltransferase } & \square \text { Unknown } \\ \text { HprK-related kinase } & \square \text { Graspetide synthetase } & \square \text { Metallo-beta-lactamase } \\ \square \text { Leader peptidase } & \square \text { Fatty acid hydroxylase } & \square \text { Tetratricopeptide repeat } \\ \square \text { Transporter } & \square \text { AhpC/TSA family } & \square \text { Methyltransferase } \\ & \end{array}$


Figure S12. Graspetide precursor peptide length distribution. Plot depicting length variation among precursor peptides from different graspetide groups. Area of the circle is proportional to the number of predicted precursors in a given group. Circles are centered on the average length, and the error bars indicate standard deviation in length.

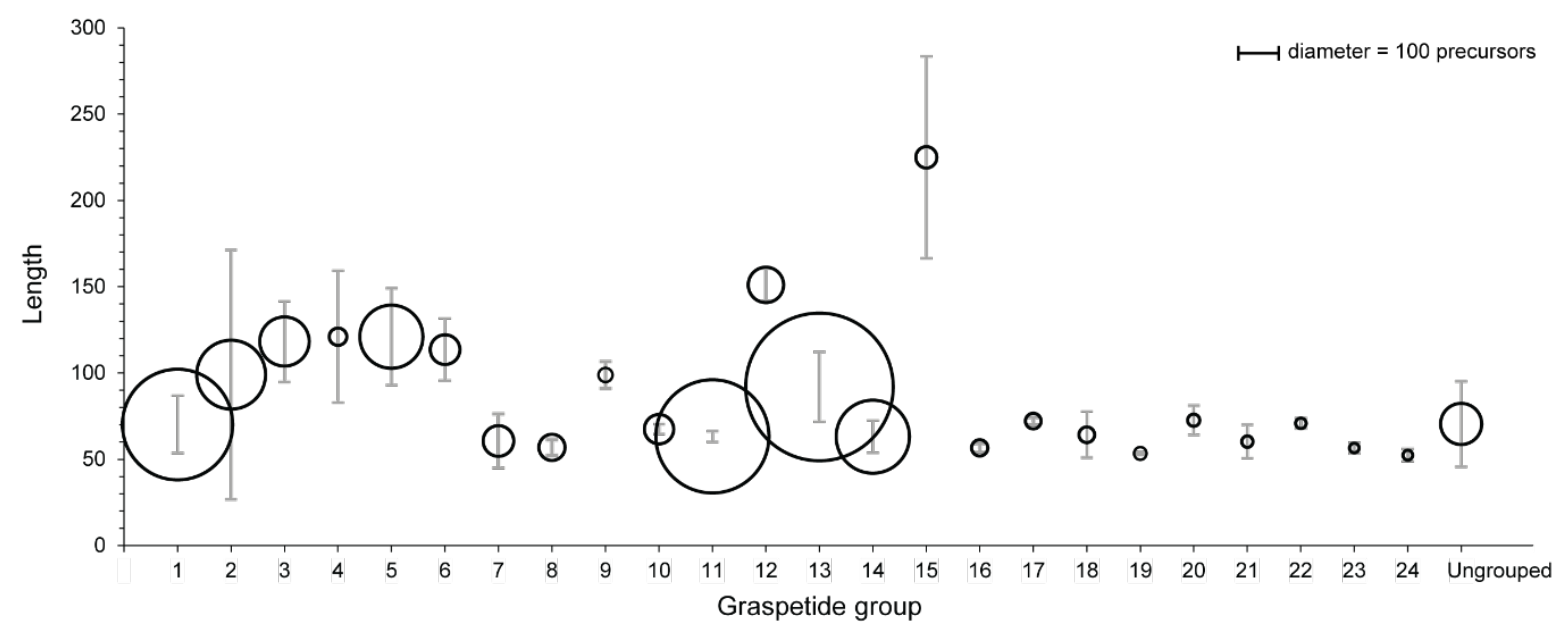


Figure S13 (shown on following two pages). Sequence similarity network of the ATP-grasp ligase superfamily. The SSN was generated using representative members of InterPro family IPR011761 and graspetide synthetases identified in this study. Nodes representing individual protein sequences are conflated at $40 \%$ identity resulting in 21,621 nodes. Node relatedness is indicated at a BLAST expectation value of $10^{-29}(\mathrm{~A})$ and $10^{-30}(\mathrm{~B})$. Protein sequences with known functions (graspeptide synthetase and non-RiPP ATP-grasp ligases annotated in Swiss-Prot ${ }^{35}$ ) are colored. BacD, Alanine--anticapsin ligase; BtrJ, Butirosin acyl-carrier protein--L-glutamate ligase; CarB, carbamoyl phosphate synthase; CofF, Coenzyme $\mathrm{F}^{-\mathrm{F} 420-2--\alpha-L-g l u t a m a t e ~ l i g a s e ; ~}$ DdlA/DdlB, D-alanine--D-alanine ligase; GshB/GshAB, Glutathione synthetase; LysX, aaminoadipate--LysW ligase; MfnD, Tyramine--L-glutamate ligase; MptN, Tetrahydro-

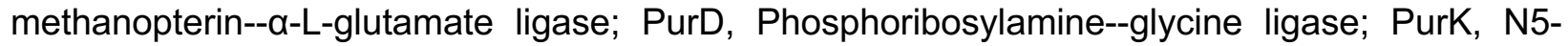
carboxyaminoimidazole ribonucleotide synthase; PurP, 5-formaminoimidazole-4-carboxamide-1(ß)-D-ribofuranosyl 5'-monophosphate synthetase; PurT, Formate-dependent phosphoribosylglycinamide formyltransferase; PycA, Pyruvate carboxylase; RimK, Ribosomal protein S6-L-glutamate ligase; RizA, L-arginine-specific L-amino acid ligase; SucC, Succinate-CoA ligase. 
A

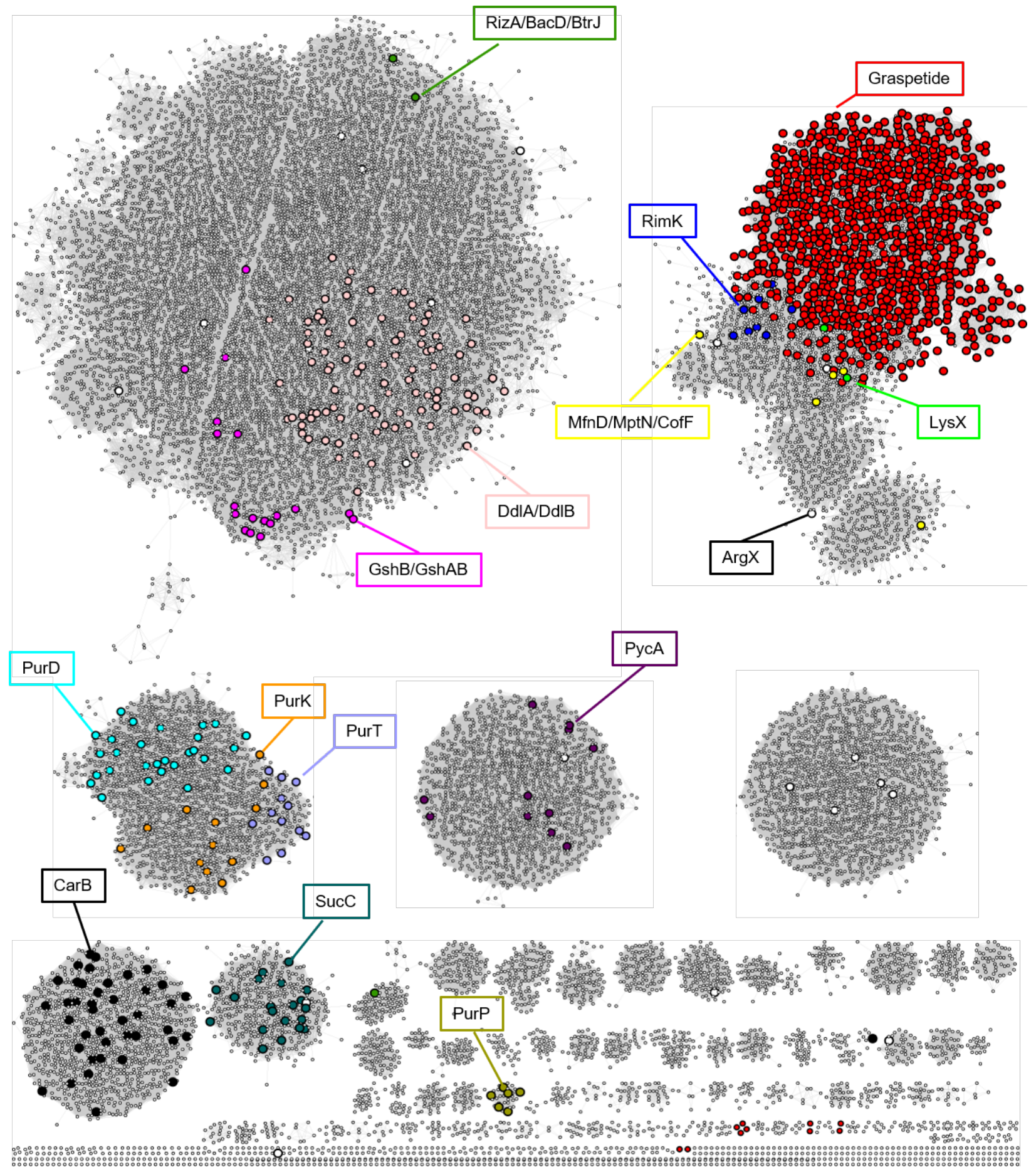


B
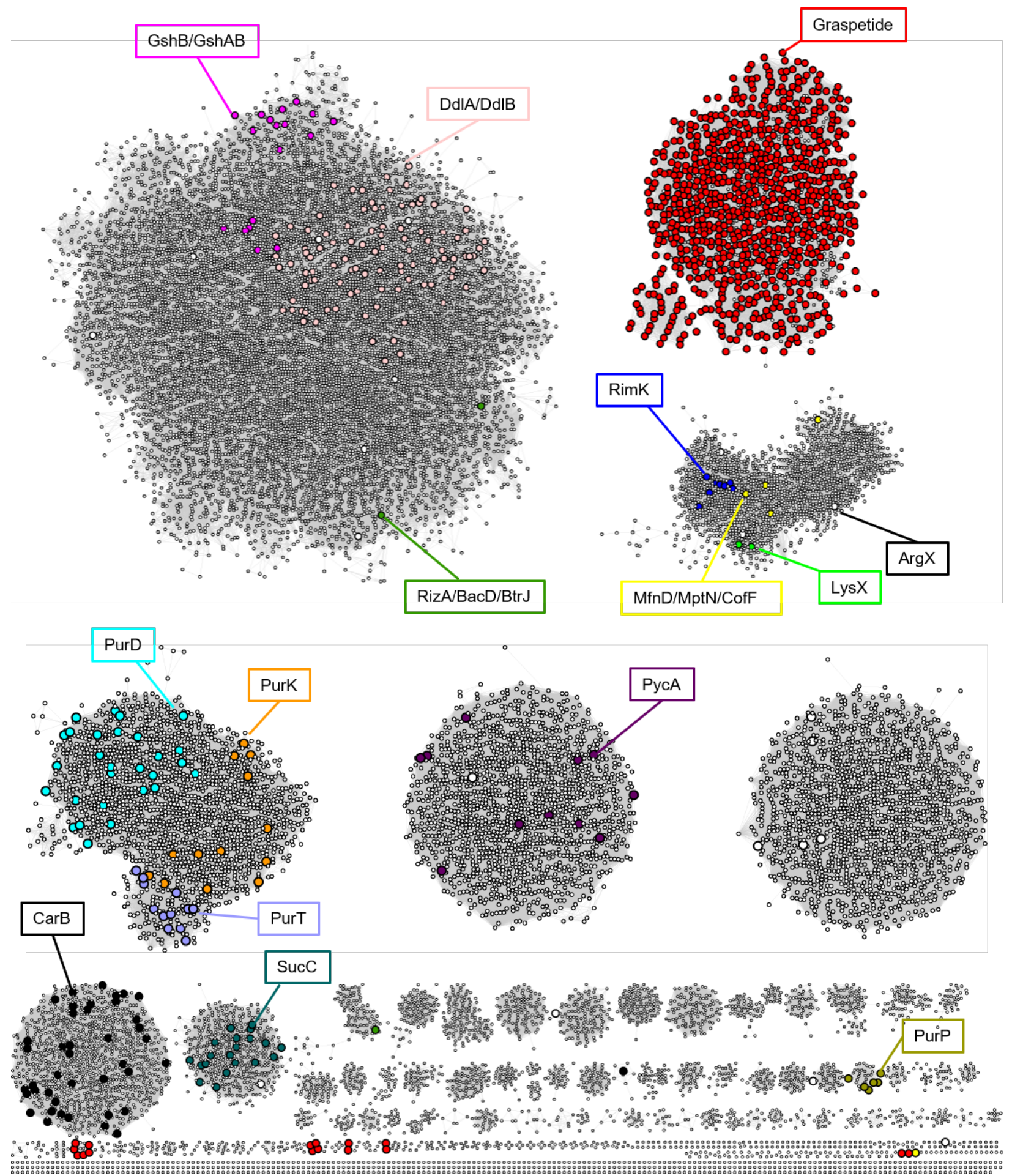
Figure S14. Maximum likelihood tree of graspetide synthetases. The tree was generated using 1,318 graspetide synthetases. The sequences represented are diversity-maximized, meaning that protein sequences above $70 \%$ identity are conflated and only include a single representative. The tree is rooted using E. coli strain K-12 RimK (ribosomal protein S6 modification protein; NCBI accession: NP_415373.1). The tree was visualized using iTOL. ${ }^{19}$ The ring is colored according to graspetide group. The data to regenerate the tree are provided in phyloXML format (Supplementary Dataset 3).

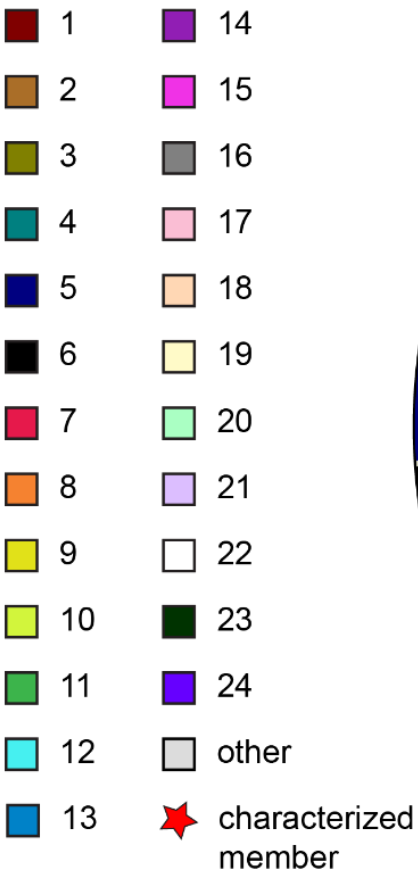

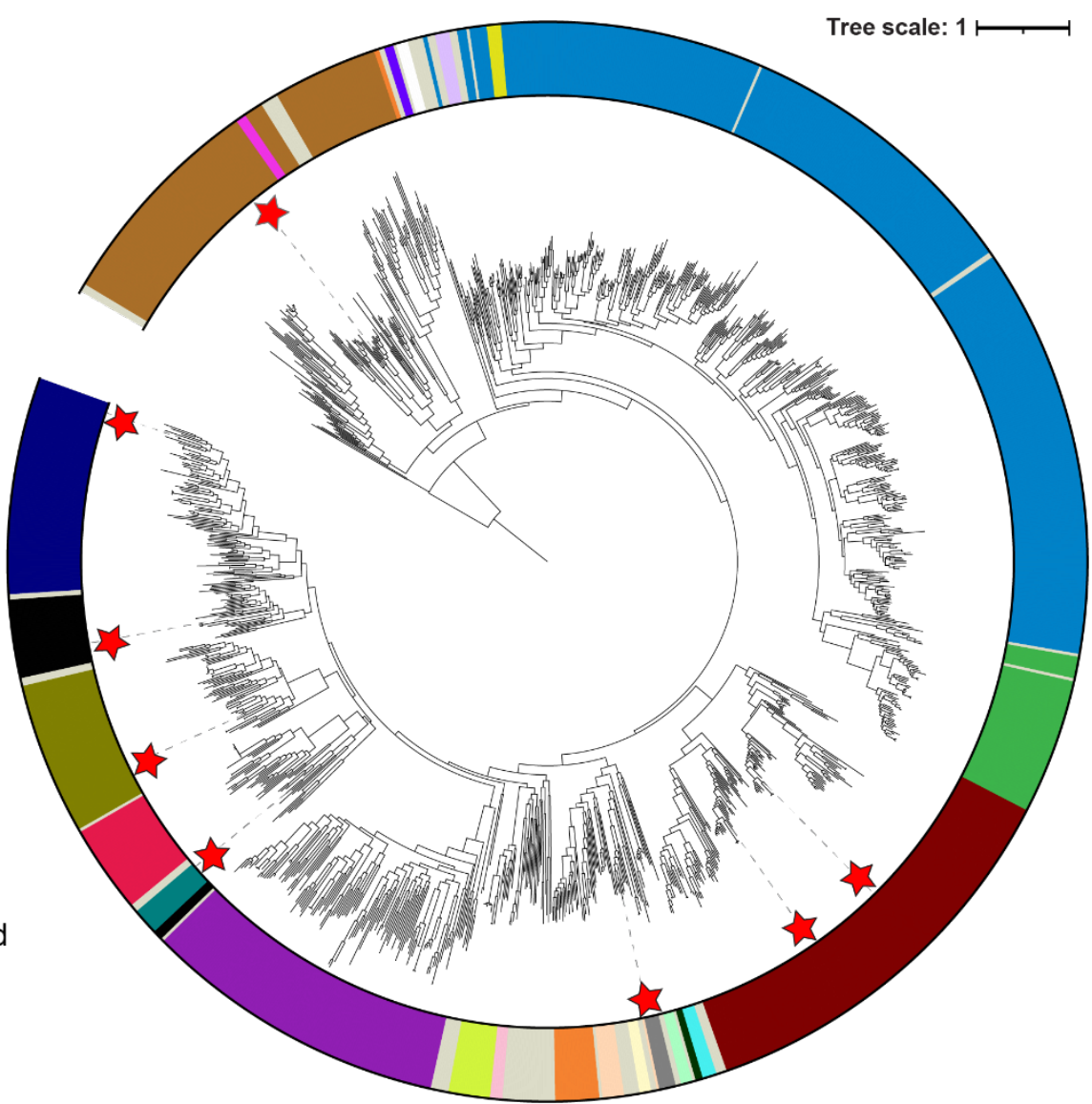


Figure S15. Analysis of $\mathbf{G}+\mathbf{C}$ content of graspetide synthetases. The $G+C$ content of graspetide synthetases from graspetide groups newly cataloged in this study. A similar analysis for groups 1-12 graspetides (previously OEPs 1-12) is referenced. ${ }^{8}$ A. GwM (group 13). B. GwS (group 14). C. Groups $15-24$ and others. D. Average difference and standard deviation between $\mathrm{G}+\mathrm{C}$ content of the genome and the graspetide synthetases for graspetide groups in A-C. graspetide synthetase genes that differed from the genomic $G+C$ content by four or more standard deviations are annotated. MAG, metagenome-assembled genome.

A

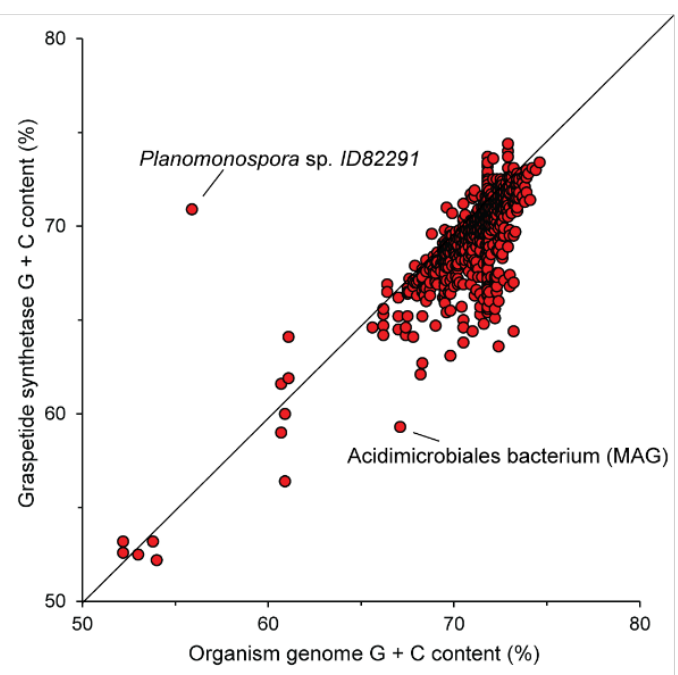

C

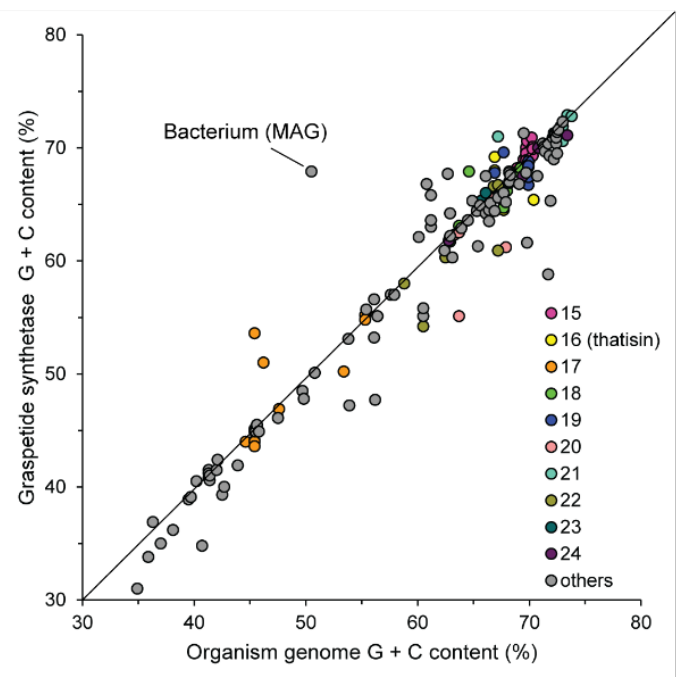

B

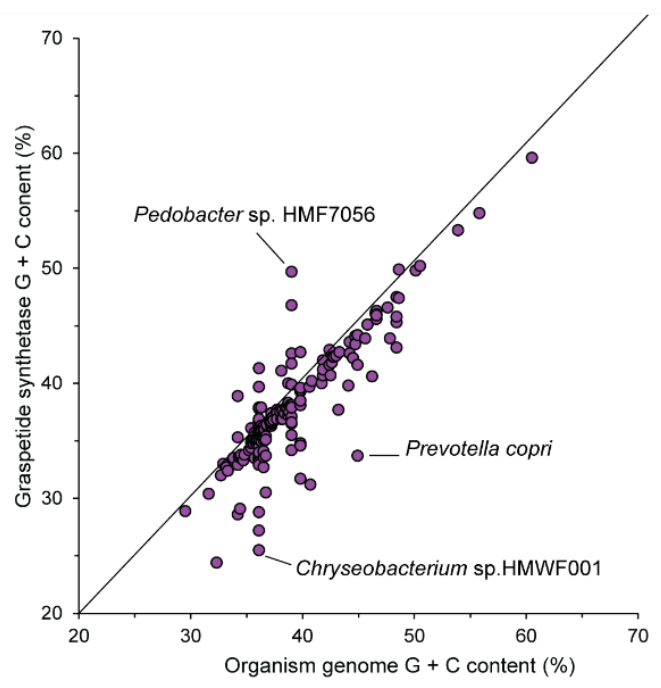

D

\begin{tabular}{lc}
\hline $\begin{array}{c}\text { Graspetide } \\
\text { group }\end{array}$ & $\begin{array}{c}\text { Average GC content } \\
\text { difference and standard } \\
\text { deviation }\end{array}$ \\
\hline $\mathbf{1 3}$ (GwM) & $1.5 \pm 1.5$ \\
$\mathbf{1 4}$ (GwS) & $1.0 \pm 2.3$ \\
$\mathbf{1 5}$ & $0.5 \pm 0.6$ \\
$\mathbf{1 6}$ (thatisin) & $1.0 \pm 2.0$ \\
$\mathbf{1 7}$ & $-0.2 \pm 3.0$ \\
$\mathbf{1 8}$ & $0.9 \pm 1.5$ \\
$\mathbf{1 9}$ & $1.0 \pm 1.5$ \\
$\mathbf{2 0}$ & $2.9 \pm 3.0$ \\
$\mathbf{2 1}$ & $0.5 \pm 1.8$ \\
$\mathbf{2 2}$ & $2.7 \pm 2.6$ \\
$\mathbf{2 3}$ & $0.5 \pm 0.4$ \\
$\mathbf{2 4}$ & $1.4 \pm 0.6$ \\
\hline
\end{tabular}


Figure S16. Co-expression of ThtA and ThtB. A. MALDI-TOF mass spectra of a TEV protease digestion of ThtA (top) and ThtA co-expressed with ThtB (bottom). The asterisks indicate ions representing laser-induced deamination. B. Same as in panel A but with the removal of the leader region of ThtA using LahT150.

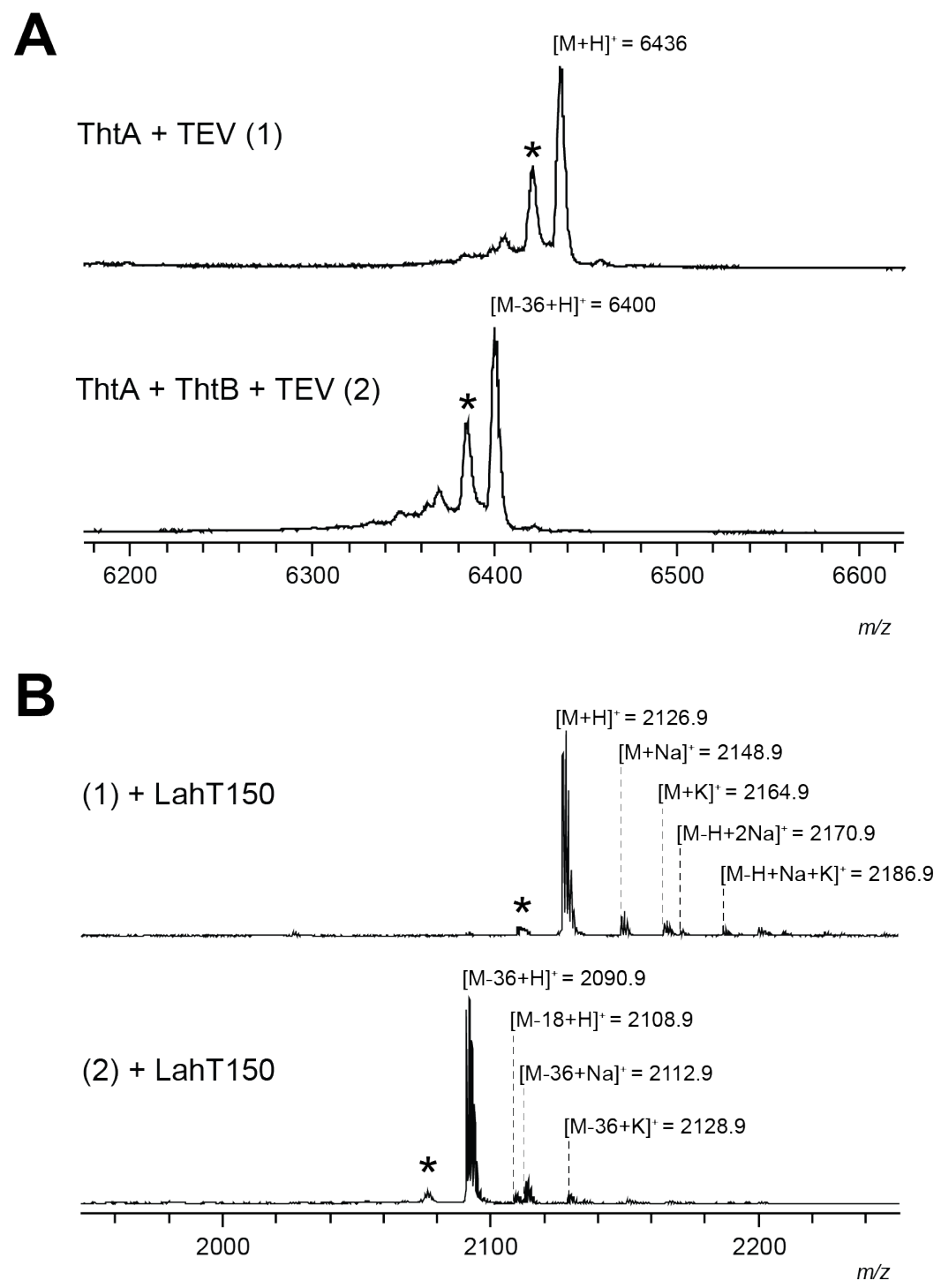


Figure S17. Sequence alignment of ThtC and LahT. Pairwise alignment of the protease domain of LahT (WP_051646490.1, residues 16-147) and ThtC (WP_057972342.1, residues 6-136) using the EMBOSS Water Webtool (https://www.ebi.ac.uk/Tools/psa/emboss water/). ${ }^{31}$ ThtC and LahT are peptidase-containing ATP-binding transporters (PCAT, Interpro: IPR005897). ${ }^{30}$ The catalytic triad residues are highlighted in yellow.

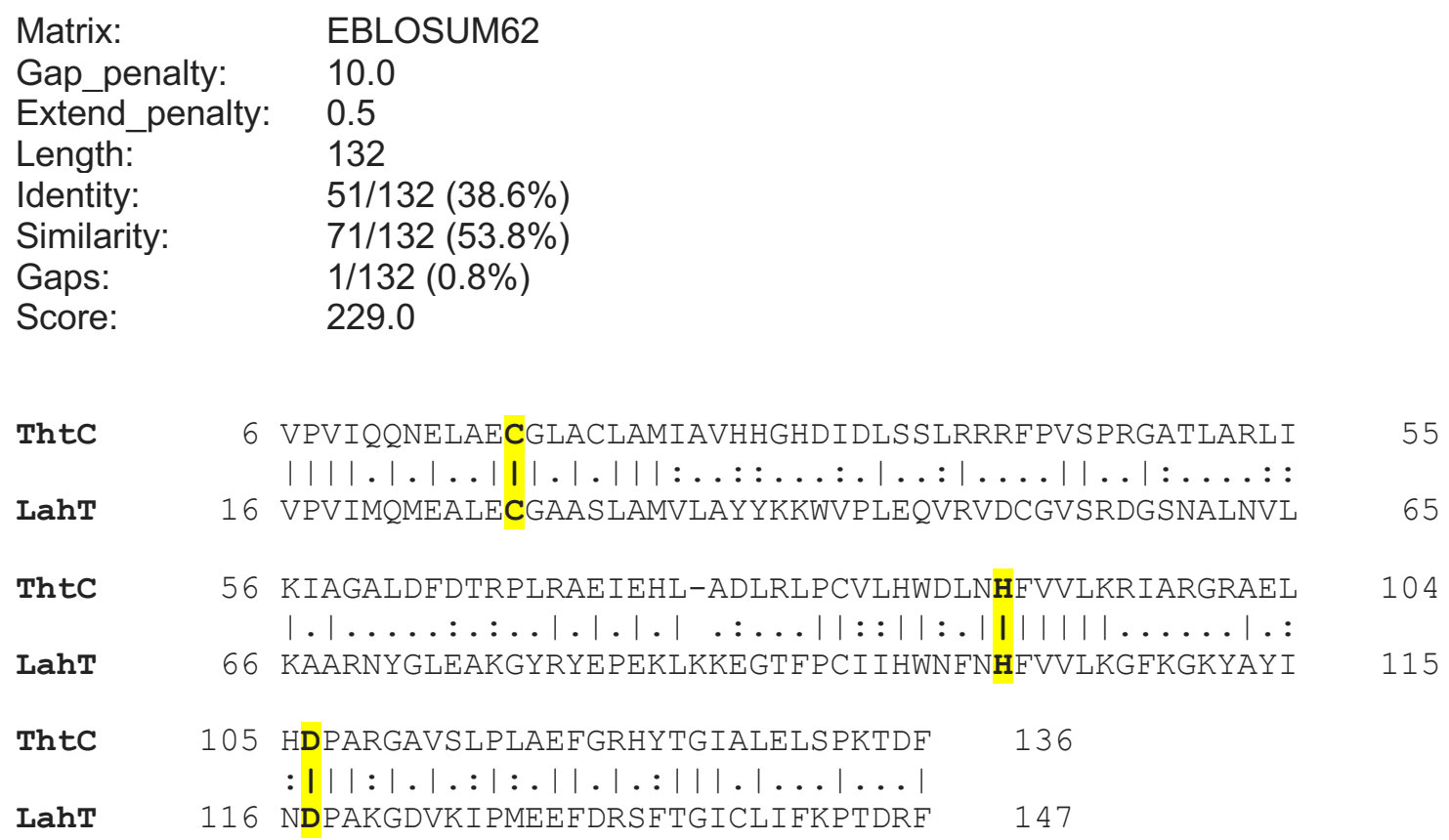


Figure S18. Thatisin and iso-thatisin. A. RP-HPLC chromatogram of LahT150-treated, modified ThtA. B. and C. HR-ESI mass spectra of thatisin and iso-thatisin, respectively. [M] = $\mathrm{C}_{89} \mathrm{H}_{131} \mathrm{~N}_{25} \mathrm{O}_{36}$.

A

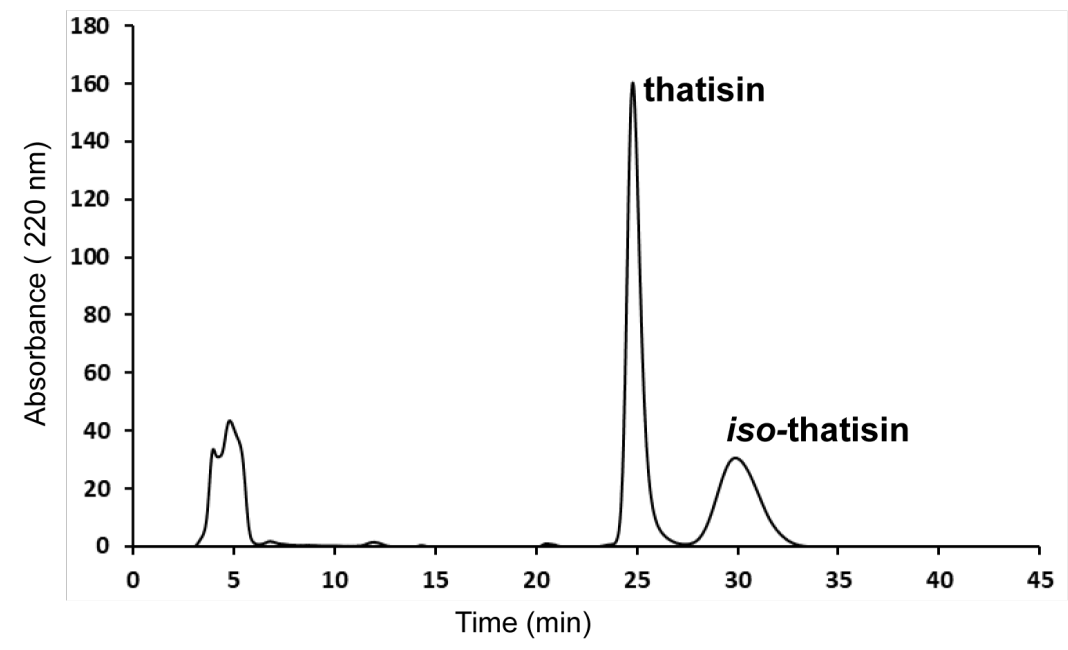

B

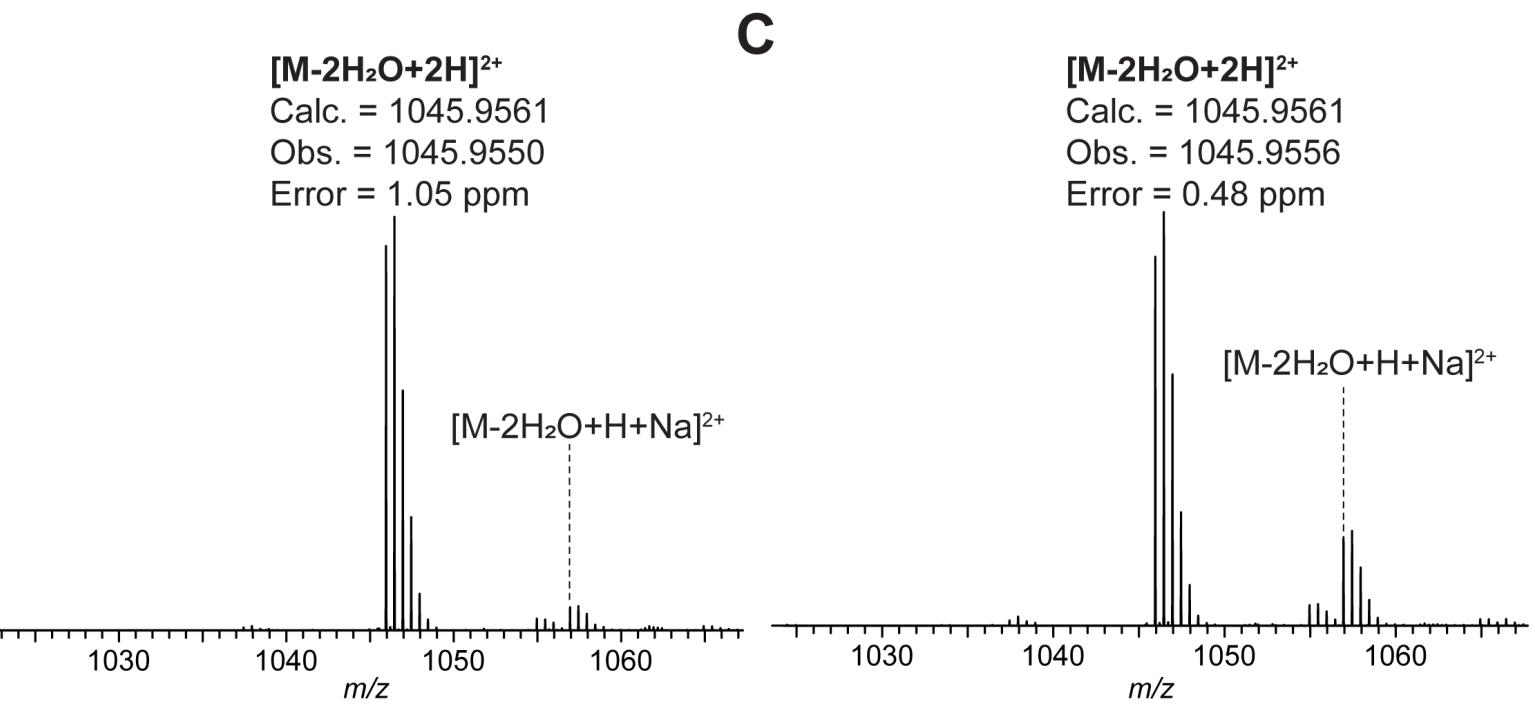


Figure S19. Thatisin: HRMS/MS.

A) HRMS/MS of thatisin. A. Fragmentation of thatisin. Observed b- and $y$-ions are annotated. Black brackets connecting Thr and Asp indicate sites of macrolactone formation. B. Annotated CID spectrum of thatisin. C. Table of daughter ion assignments.

A

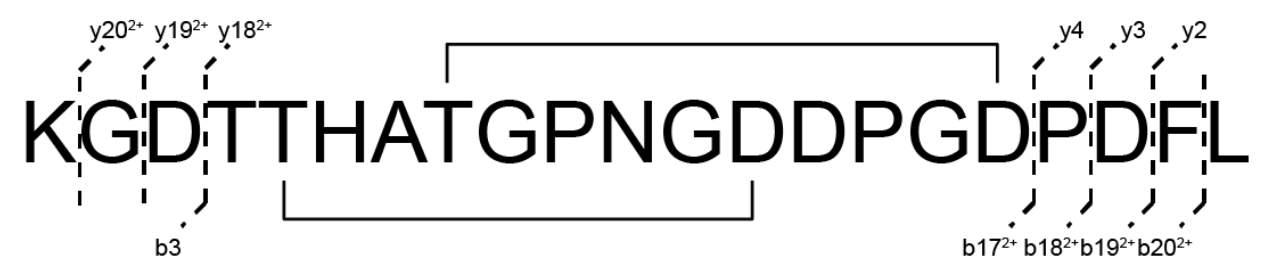

B

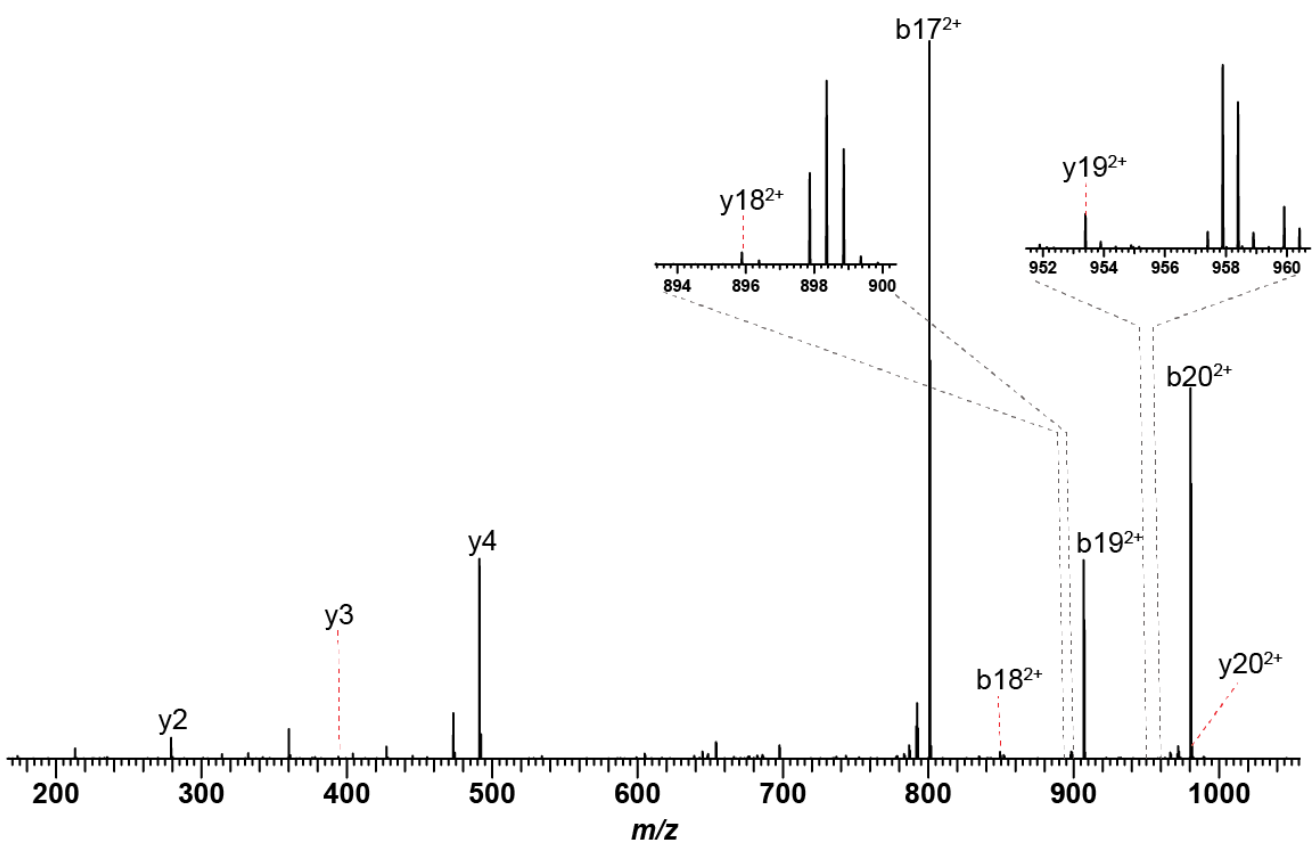

C

\begin{tabular}{cccc}
\hline lon & Calc. Mass & Obs. Mass & Error (ppm) \\
\hline$[\mathbf{M + 3 H}]^{3+}$ & 697.6399 & 697.6403 & 0.7 \\
\hline b3 & 301.1507 & 301.1496 & 3.4 \\
b17 $^{\mathbf{2 +}}$ & 800.8348 & 800.8322 & 3.2 \\
b18 $^{2+}$ & 849.3611 & 849.3593 & 2.1 \\
b19 $^{2+}$ & 906.8746 & 906.8729 & 1.9 \\
b20 $^{2+}$ & 980.4088 & 980.4067 & 2.1 \\
$\mathbf{y 2}$ & 279.1703 & 279.1693 & 3.8 \\
$\mathbf{y 3}$ & 394.1973 & 394.1963 & 2.5 \\
$\mathbf{y 4}$ & 491.2500 & 491.2492 & 1.8 \\
$\mathbf{y 1 8 ^ { 2 + }}$ & 895.8845 & 895.8833 & 1.3 \\
$\mathbf{y 1 9}^{2+}$ & 953.3979 & 953.3948 & 3.3 \\
$\mathbf{y 2 0}^{2+}$ & 981.9087 & 981.9065 & 2.2 \\
\hline & & &
\end{tabular}


B) HRMS/MS of fully methanolized thatisin. A. Fragmentation of fully methanolized thatisin. Observed b- and y-ions are annotated. Purple indicates an Asp methyl ester. B. Annotated CID spectrum of fully methanolized thatisin. C. Table of daughter ion assignments.

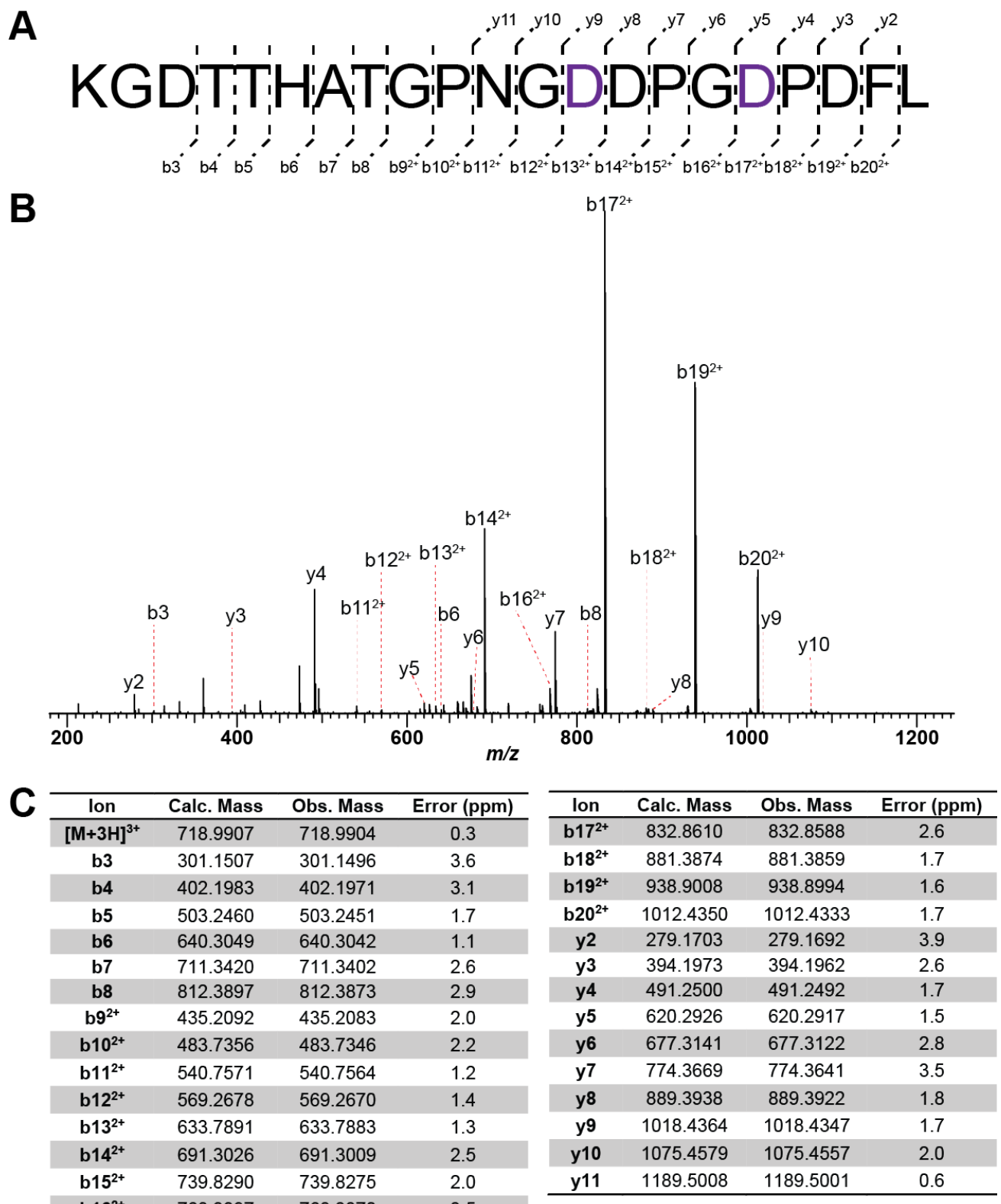


C) HRMS/MS of partially methanolized thatisin. A. Fragmentation of partially methanolized thatisin. Observed $b$ - and $y$-ions are annotated. Purple indicates an Asp methyl ester. Black bracket connecting Thr and Asp indicates intact macrolactone. B. Annotated CID spectrum of partially methanolized thatisin. C. Table of daughter ion assignments.

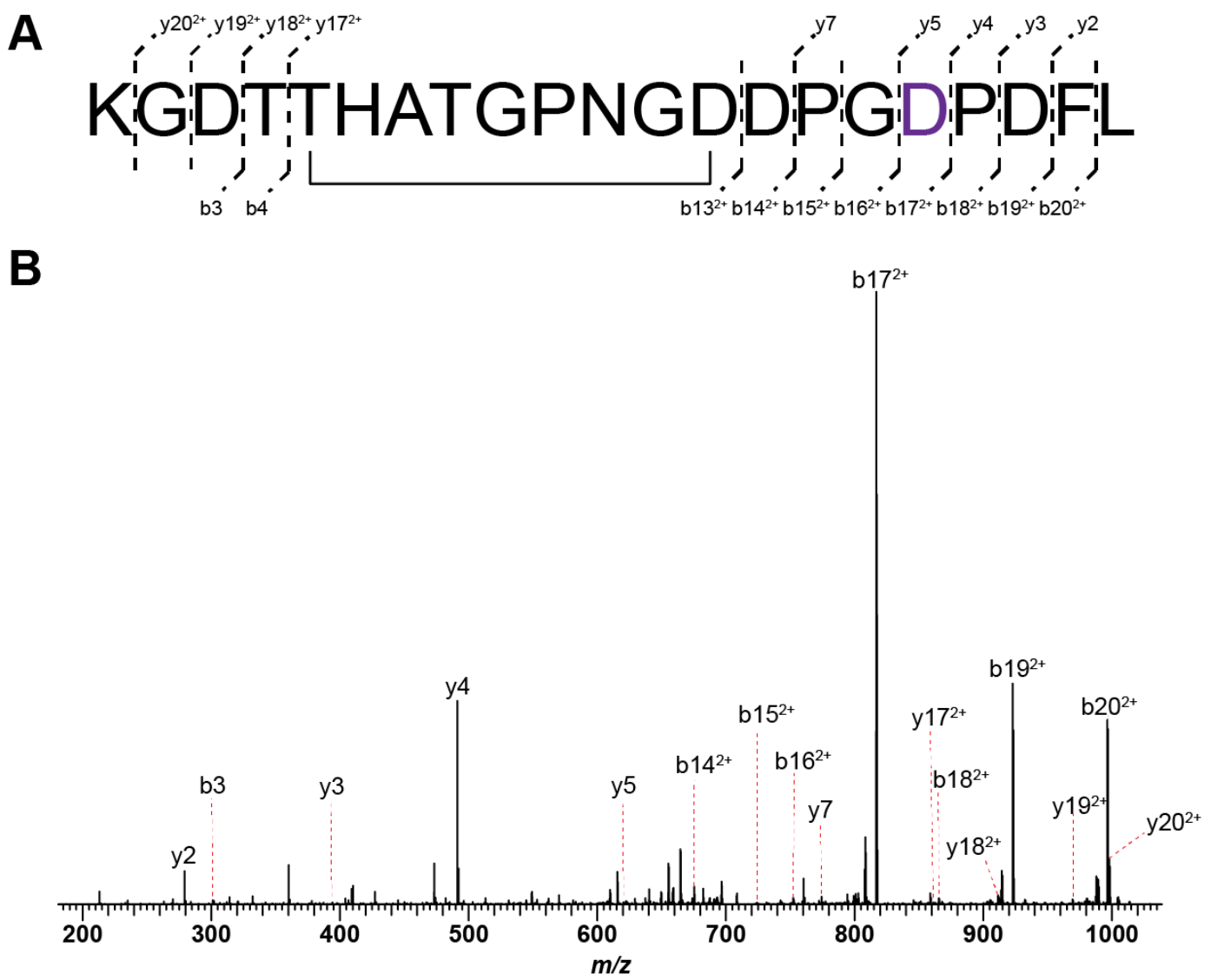

\begin{tabular}{cccc}
\hline Ion & Calc. Mass & Obs. Mass & Error $(\mathbf{p p m})$ \\
\hline$[\mathbf{M + 3 H}]^{3+}$ & 708.3153 & 708.3150 & 0.4 \\
\hline b3 & 301.1507 & 301.1495 & 3.9 \\
\hline b4 & 402.1983 & 402.1971 & 3.0 \\
\hline b13 $^{2+}$ & 617.7760 & 617.7750 & 1.6 \\
\hline b14 $^{2+}$ & 675.2895 & 675.2877 & 2.6 \\
\hline b15 $^{2+}$ & 723.8158 & 723.8142 & 2.2 \\
\hline b16 $^{2+}$ & 752.3266 & 752.3238 & 3.8 \\
\hline b17 $^{2+}$ & 816.8479 & 816.8453 & 3.1 \\
\hline b18 $^{2+}$ & 865.3743 & 865.3726 & 1.9 \\
\hline b19 $^{2+}$ & 922.8877 & 922.8858 & 2.1 \\
\hline b20 $^{2+}$ & 996.4219 & 996.4198 & 2.2 \\
\hline
\end{tabular}

\begin{tabular}{cccc}
\hline Ion & Calc. Mass & Obs. Mass & Error $(\mathbf{p p m})$ \\
\hline y2 & 279.1703 & 279.1692 & 4.1 \\
y3 & 394.1973 & 394.1962 & 2.7 \\
y4 & 491.2500 & 491.2491 & 1.9 \\
y5 & 620.2926 & 620.2919 & 1.1 \\
y7 & 774.3669 & 774.3642 & 3.4 \\
y17 $^{2+}$ & 861.3737 & 861.3720 & 2.0 \\
y18 $^{2+}$ & 911.8976 & 911.8956 & 2.1 \\
y19 $^{2+}$ & 969.4110 & 969.4089 & 2.2 \\
y20 $^{2+}$ & 997.9218 & 997.9194 & 2.3 \\
\hline
\end{tabular}


Figure S20. MALDI-TOF-MS analysis of LahT150-digested ThtA-variants. A. Asp to Alasubstituted variants. B. Thr to Ala-substituted variants. Variants involving the macrolactone acceptor and donor positions in wild-type thatisin are in blue and green, respectively.
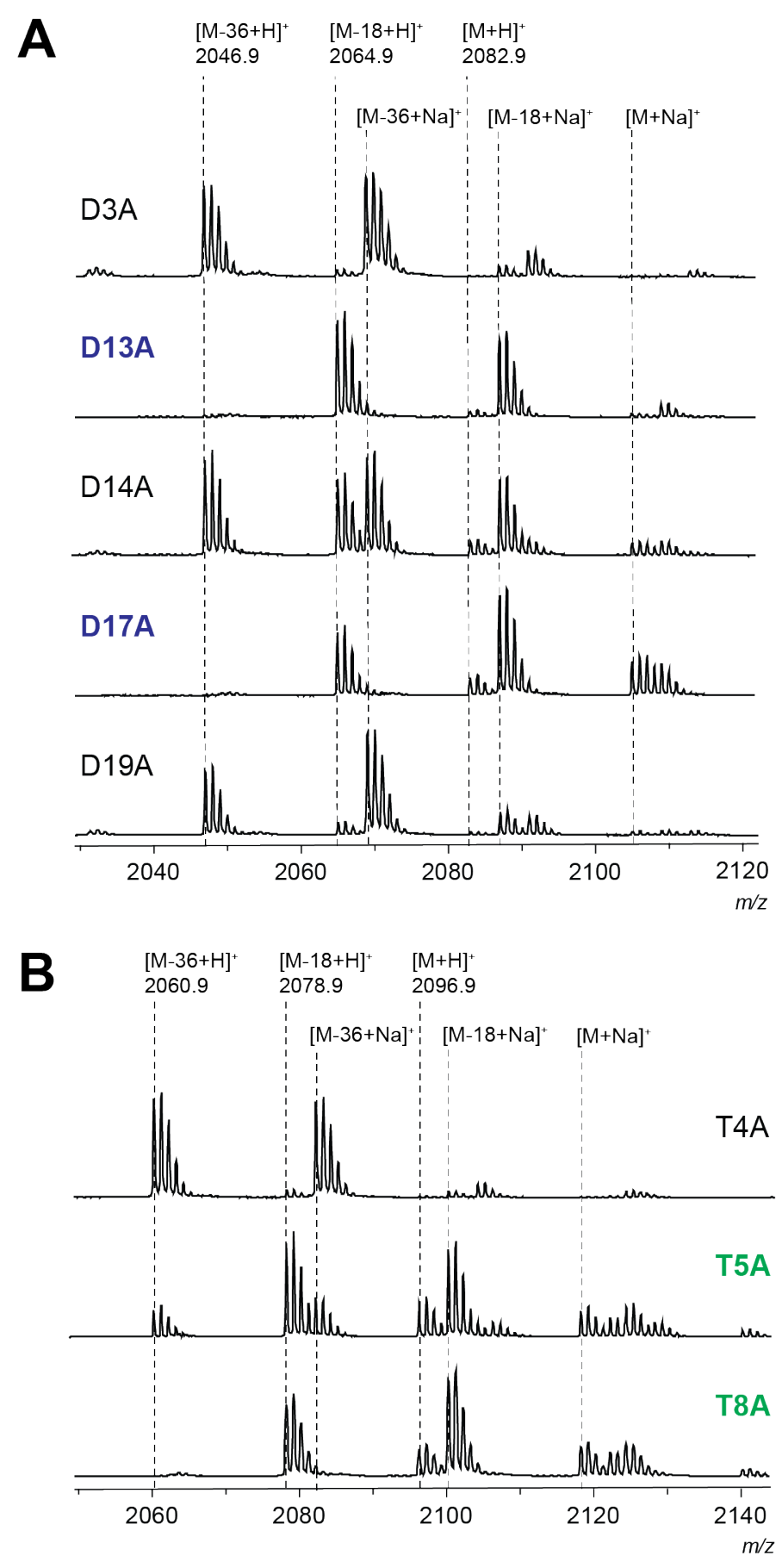
Table S4. Summary of thatisin macrolactone variants. Products formed upon co-expression of Tht $A$ variants with ThtB are shown with wild-type macrolactone donors and acceptors in green and blue, respectively. Ala substitutions are red. $L P$ refers to leader peptide. The number in parentheses indicates the number of isomers observed by reverse-phase HPLC. HRMS/MS data is available in Figures S19, S21-30.

\begin{tabular}{|c|c|c|}
\hline \multirow{2}{*}{ ThtA variant } & \multicolumn{2}{|c|}{ Macrolactone(s) observed } \\
\hline & One & Two \\
\hline LP-KGDTTHATGPNGDDPGDPDFL & Thr5-Asp13 (2) & Thr5-Asp13; Thr8-Asp17 (2) \\
\hline LP-KGATTHATGPNGDDPGDPDFL & Thr5-Asp13 & Thr5-Asp13; Thr8-Asp17 (2) \\
\hline LP-KGDATHATGPNGDDPGDPDFL & not observed & Thr5-Asp13; Thr8-Asp17 (2) \\
\hline \multirow{3}{*}{ LP-KGDTAHATGPNGDDPGDPDFL } & Thr4-Asp13 & \multirow{3}{*}{ Thr4-Asp13; Thr8-Asp17 } \\
\hline & Thr4-Asp14 & \\
\hline & Thr8-Asp17 & \\
\hline LP-KGDTTHAAGPNGDDPGDPDFL & Thr5-Asp13 (2) & not observed \\
\hline \multirow{2}{*}{ LP-KGDTTHATGPNGADPGDPDFL } & Thr5-Asp14 & \multirow{2}{*}{ not observed } \\
\hline & Thr8-Asp17 & \\
\hline LP-KGDTTHATGPNGDAPGDPDFL & Thr5-Asp13 & Thr5-Asp13; Thr8-Asp17 \\
\hline LP-KGDTTHATGPNGDDPGAPDFL & Thr5-Asp13 (2) & not observed \\
\hline LP-KGDTTHATGPNGDDPGDPAFL & Thr5-Asp13 (2) & Thr5-Asp13; Thr8-Asp17 (2) \\
\hline
\end{tabular}


Figure S21. Wild-type ThtA-singly modified products: HRMS/MS.

A) HRMS/MS of singly modified product-1 produced by wild-type ThtA. A. Fragmentation of singly modified product- 1 formed upon co-expression of ThtA with ThtB. Observed b- and y-ions are annotated. The black bracket connecting Thr and Asp indicates the site of macrolactone formation. B. Annotated CID spectrum of singly modified product-1. C. Table of daughter ion assignments.

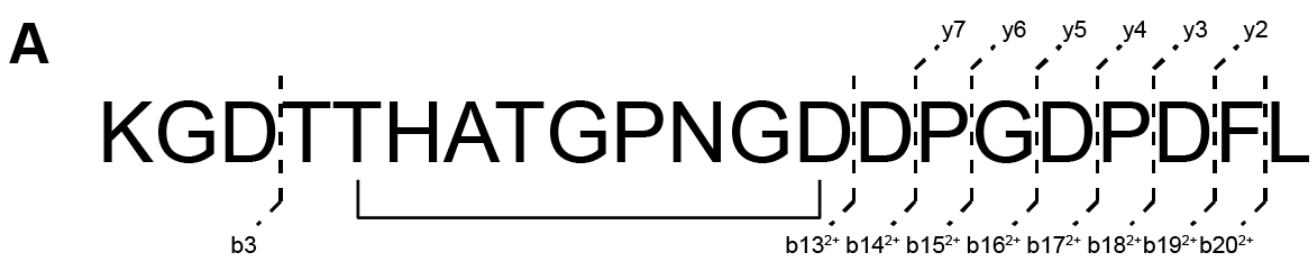

B

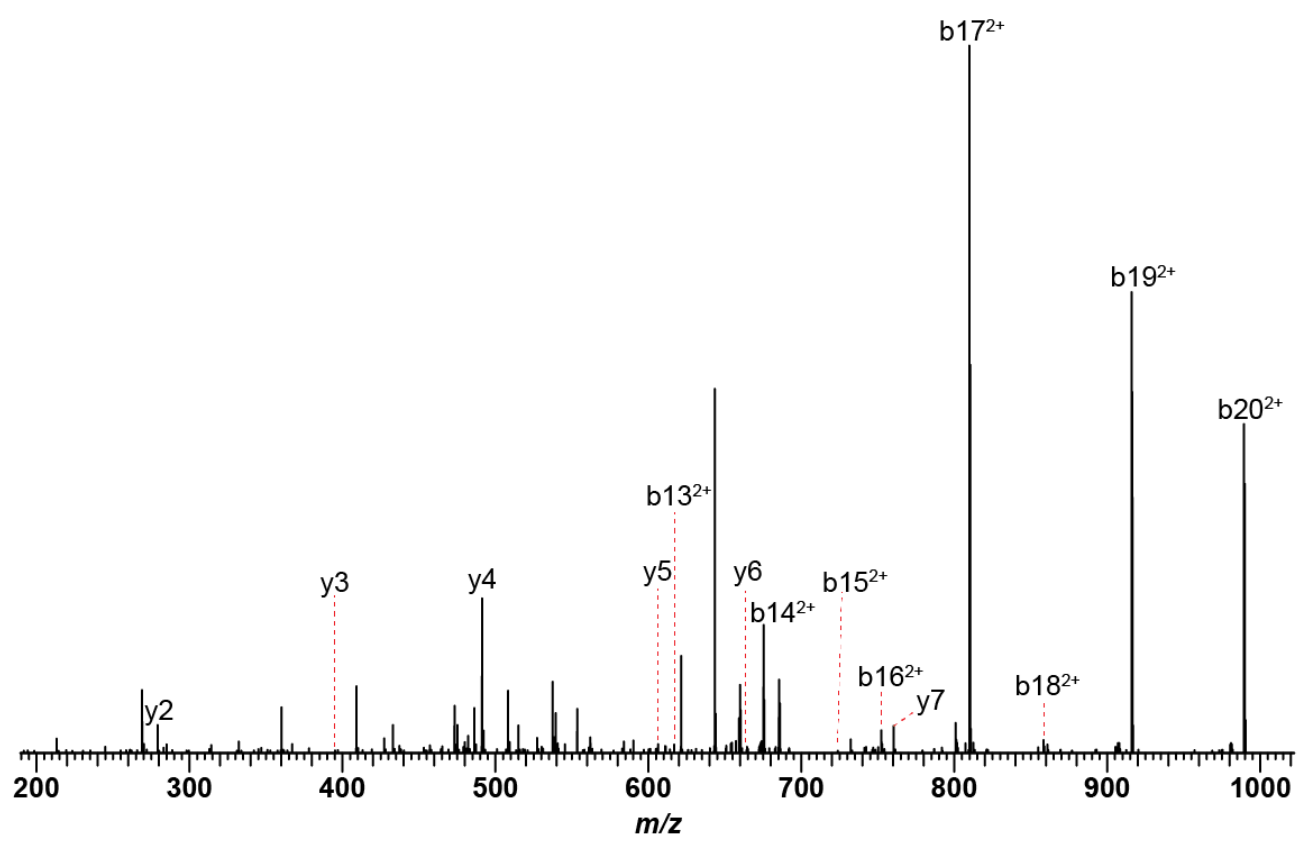

\begin{tabular}{cccc}
\hline Ion & Calc. Mass & Obs. Mass & $\begin{array}{c}\text { Error } \\
\text { (ppm) }\end{array}$ \\
\hline$\left[\mathbf{M + 3 H ] ^ { 3 + }}\right.$ & 703.6434 & 703.6432 & 0.3 \\
\hline b3 & 301.1507 & 301.1493 & 4.6 \\
\hline b13 $^{2+}$ & 617.7760 & 617.7752 & 1.3 \\
\hline b14 $^{2+}$ & 675.2895 & 675.2876 & 2.8 \\
\hline b15 $^{2+}$ & 723.8158 & 723.8143 & 2.2 \\
\hline b16 $^{2+}$ & 752.3266 & 752.3236 & 3.9 \\
\hline b17 $^{2+}$ & 809.8400 & 809.8373 & 3.4 \\
\hline b18 $^{2+}$ & 858.3664 & 858.3645 & 2.2 \\
\hline b19 $^{2+}$ & 915.8799 & 915.8777 & 2.4 \\
\hline b20 $^{2+}$ & 989.4141 & 989.4116 & 2.5 \\
\hline
\end{tabular}

\begin{tabular}{cccc}
\hline Ion & Calc. Mass & Obs. Mass & $\begin{array}{c}\text { Error } \\
\text { (ppm) }\end{array}$ \\
\hline y2 & 279.1703 & 279.1692 & 4.2 \\
y3 & 394.1973 & 394.1962 & 2.8 \\
y4 & 491.2500 & 491.2491 & 2.0 \\
y5 & 606.2770 & 606.2761 & 1.4 \\
y6 & 663.2984 & 663.2976 & 1.2 \\
\hline y7 & 760.3512 & 760.3483 & 3.8 \\
\hline
\end{tabular}


B) HRMS/MS of methanolized singly modified product-1 produced by wild-type ThtA. A. Fragmentation of methanolized singly modified product-1 formed upon co-expression of ThtA with ThtB. Observed b- and y-ions are annotated. Purple indicates an Asp methyl ester. B. Annotated CID spectrum of fully methanolized singly modified product-1. C. Table of daughter ion assignments.

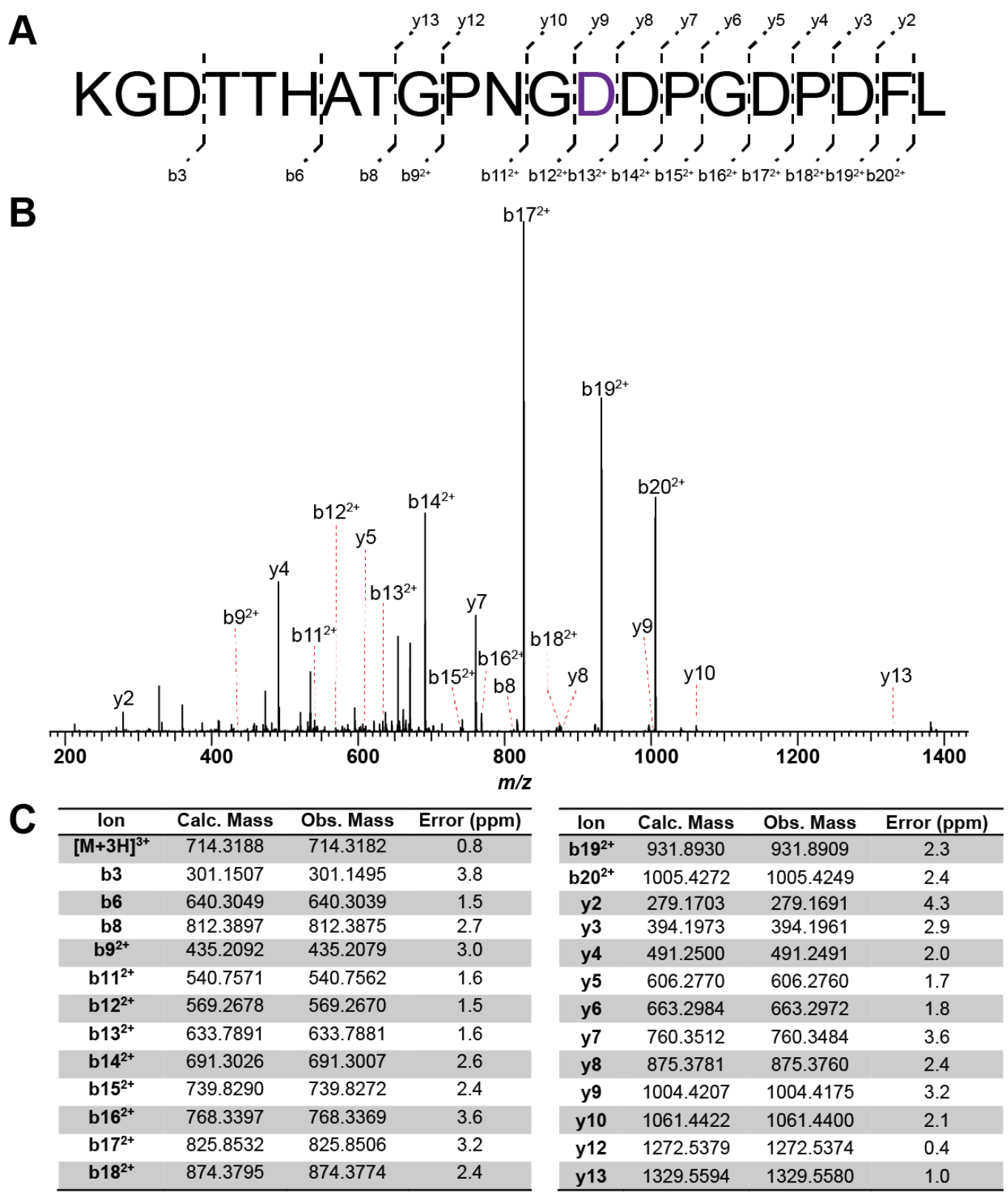


C) HRMS/MS of singly modified product-2 produced by wild-type ThtA. A. Fragmentation of singly modified product- 2 formed upon co-expression of ThtA with ThtB. Observed $b$ - and y-ions are annotated. The black bracket connecting Thr and Asp indicates the site of macrolactone formation. B. Annotated CID spectrum of singly modified product-2. C. Table of daughter ion assignments.

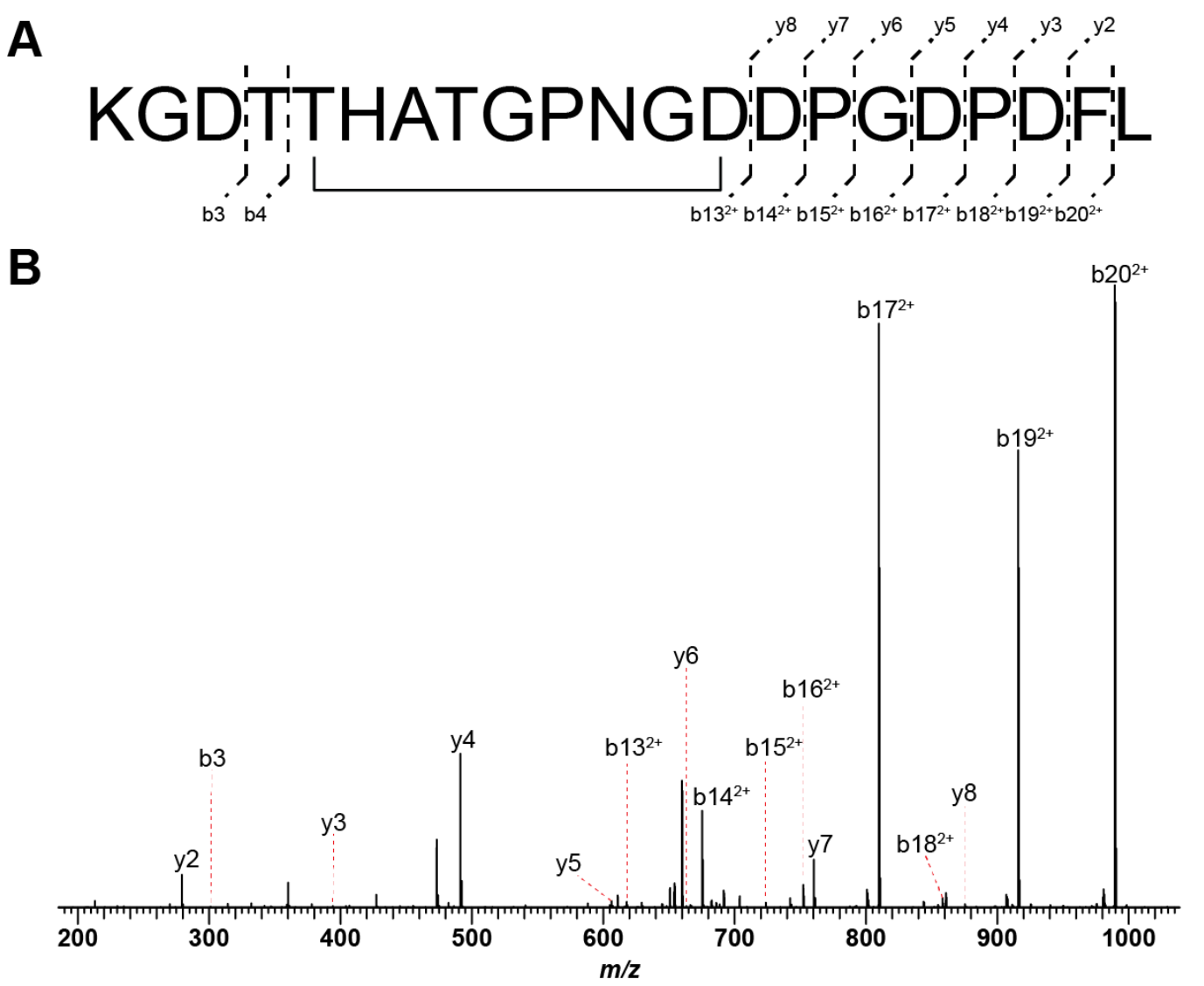

\begin{tabular}{cccc}
\hline Ion & Calc. Mass & Obs. Mass & Error $(\mathbf{p p m})$ \\
\hline$[\mathbf{M + 3 H}]^{3+}$ & 703.6434 & 703.6431 & 0.3 \\
b3 & 301.1507 & 301.1496 & 3.6 \\
\hline b4 & 402.1983 & 402.1973 & 2.6 \\
\hline b13 $^{2+}$ & 617.7760 & 617.7751 & 1.5 \\
b14 $^{2+}$ & 675.2895 & 675.2877 & 2.6 \\
\hline b15 $^{2+}$ & 723.8158 & 723.8143 & 2.1 \\
\hline b16 $^{2+}$ & 752.3266 & 752.3238 & 3.7 \\
\hline b17 $^{2+}$ & 809.8400 & 809.8374 & 3.3 \\
\hline b18 $^{2+}$ & 858.3664 & 858.3648 & 1.9 \\
\hline b19 $^{2+}$ & 915.8799 & 915.8780 & 2.1 \\
\hline b20 $^{2+}$ & 989.4141 & 989.4120 & 2.1 \\
\hline
\end{tabular}

\begin{tabular}{cccc}
\hline Ion & Calc. Mass & Obs. Mass & Error $(\mathbf{p p m})$ \\
\hline y2 & 279.1703 & 279.1692 & 4.0 \\
y3 & 394.1973 & 394.1962 & 2.7 \\
y4 & 491.2500 & 491.2491 & 1.9 \\
y5 & 606.2770 & 606.2762 & 1.3 \\
y6 & 663.2984 & 663.2975 & 1.4 \\
y7 & 760.3512 & 760.3484 & 3.7 \\
y8 & 875.3781 & 875.3764 & 2.0 \\
\hline
\end{tabular}


D) HRMS/MS of methanolized singly modified product-2 produced by wild-type ThtA. A. Fragmentation of methanolized singly modified product-2 formed upon co-expression of ThtA with ThtB. Observed b- and y-ions are annotated. Purple indicates an Asp methyl ester. B. Annotated CID spectrum of fully methanolized singly modified product-2. C. Table of daughter ion assignments.

A

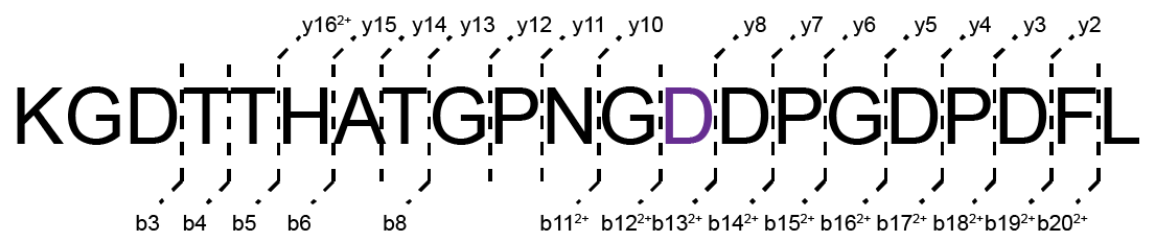

B

b8

$\mathrm{b} 11^{2+} \mathrm{b} 12^{2+} \mathrm{b} 13^{2+} \mathrm{b} 14^{2+} \mathrm{b} 15^{2+} \mathrm{b} 16^{2+} \mathrm{b} 17^{2+} \mathrm{b} 18^{2+} \mathrm{b} 19^{2+} \mathrm{b} 2 \mathrm{O}^{2+}$

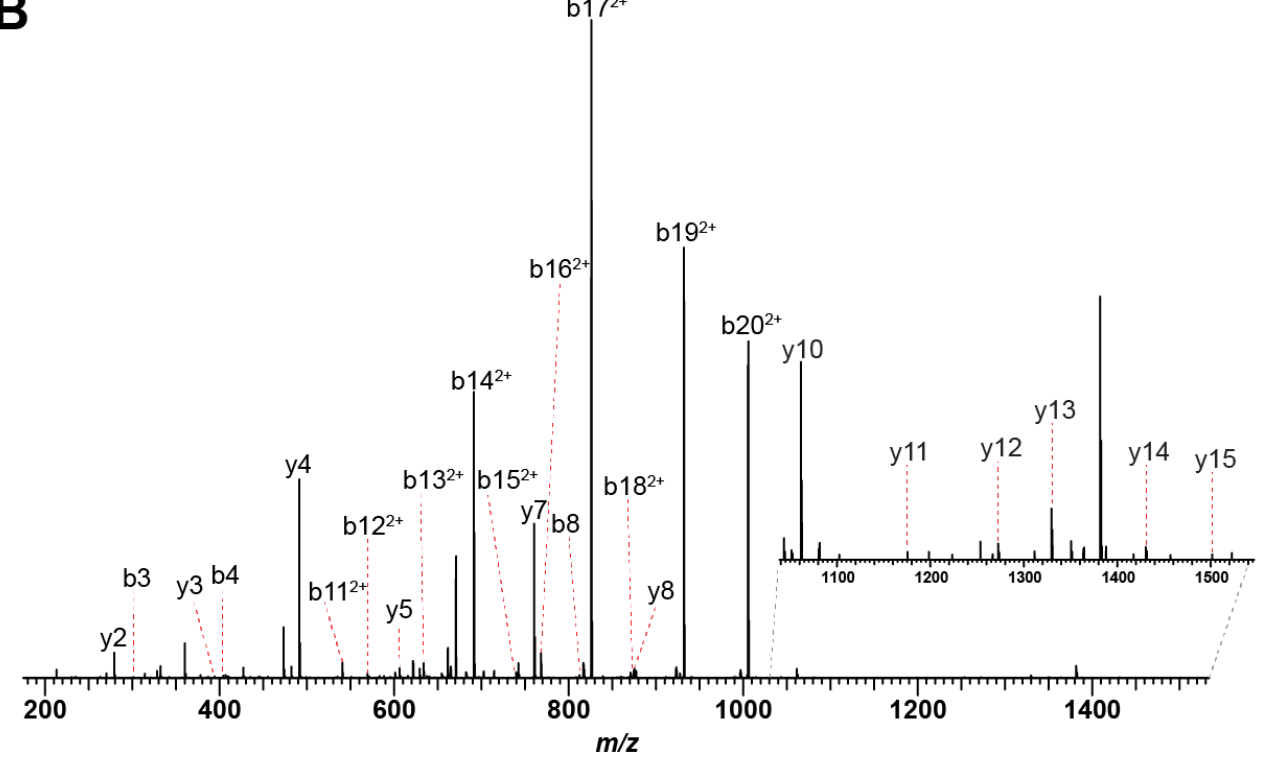

\begin{tabular}{|c|c|c|c|}
\hline Ion & Calc. Mass & Obs. Mass & Error (ppm) \\
\hline$[\mathrm{M}+3 \mathrm{H}]^{3+}$ & 714.3188 & 714.3184 & 0.5 \\
\hline b3 & 301.1507 & 301.1494 & 4.1 \\
\hline b4 & 402.1983 & 402.1972 & 2.8 \\
\hline b5 & 503.2460 & 503.2449 & 2.1 \\
\hline b6 & 640.3049 & 640.3040 & 1.4 \\
\hline b8 & 812.3897 & 812.3875 & 2.7 \\
\hline b11 ${ }^{2+}$ & 540.7571 & 540.7563 & 1.5 \\
\hline b12 ${ }^{2+}$ & 569.2678 & 569.2669 & 1.6 \\
\hline b13 ${ }^{2+}$ & 633.7891 & 633.7882 & 1.4 \\
\hline b14 ${ }^{2+}$ & 691.3026 & 691.3009 & 2.4 \\
\hline b15 ${ }^{2+}$ & 739.8290 & 739.8272 & 2.3 \\
\hline b16 ${ }^{2+}$ & 768.3397 & 768.3370 & 3.5 \\
\hline b17 ${ }^{2+}$ & 825.8532 & 825.8506 & 3.0 \\
\hline b18 ${ }^{2+}$ & 874.3795 & 874.3775 & 2.3 \\
\hline b1 $19^{2+}$ & 931.8930 & 931.8910 & 2.2 \\
\hline
\end{tabular}

\begin{tabular}{|c|c|c|c|}
\hline Ion & Calc. Mass & Obs. Mass & Error (ppm) \\
\hline b20 $2^{2+}$ & 1005.4272 & 1005.4249 & 2.3 \\
\hline y2 & 279.1703 & 279.1692 & 4.2 \\
\hline y3 & 394.1973 & 394.1961 & 3.0 \\
\hline y4 & 491.2500 & 491.2491 & 2.0 \\
\hline y5 & 606.2770 & 606.2761 & 1.5 \\
\hline y6 & 663.2984 & 663.2975 & 1.4 \\
\hline y7 & 760.3512 & 760.3485 & 3.6 \\
\hline y8 & 875.3781 & 875.3762 & 2.2 \\
\hline y10 & 1061.4422 & 1061.4402 & 1.9 \\
\hline y11 & 1175.4851 & 1175.4836 & 1.3 \\
\hline y12 & 1272.5379 & 1272.5353 & 2.0 \\
\hline y13 & 1329.5594 & 1329.5574 & 1.5 \\
\hline y14 & 1430.6070 & 1430.6052 & 1.3 \\
\hline y15 & 1501.6441 & 1501.6406 & 2.4 \\
\hline$y 16^{2+}$ & 819.8552 & 819.8525 & 3.3 \\
\hline
\end{tabular}


Figure S22. ThtA-D3A variant-modified products: HRMS/MS.

A) HRMS/MS of doubly modified product-1 formed by ThtA-D3A variant. A. Fragmentation of doubly modified product-1 of ThtA-D3A variant. Observed b- and y-ions are annotated. Green denotes the substituted position. Black brackets connecting Thr and Asp indicate sites of macrolactone formation. B. Annotated CID spectrum of doubly modified product-1 of ThtA-D3A variant. C. Table of daughter ion assignments.

A

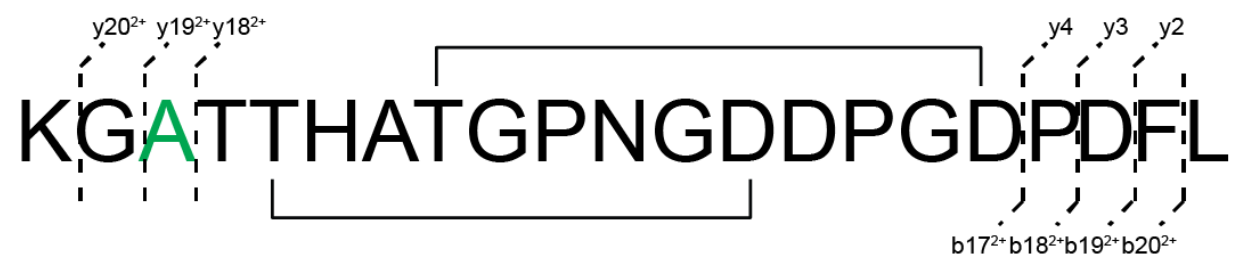

B

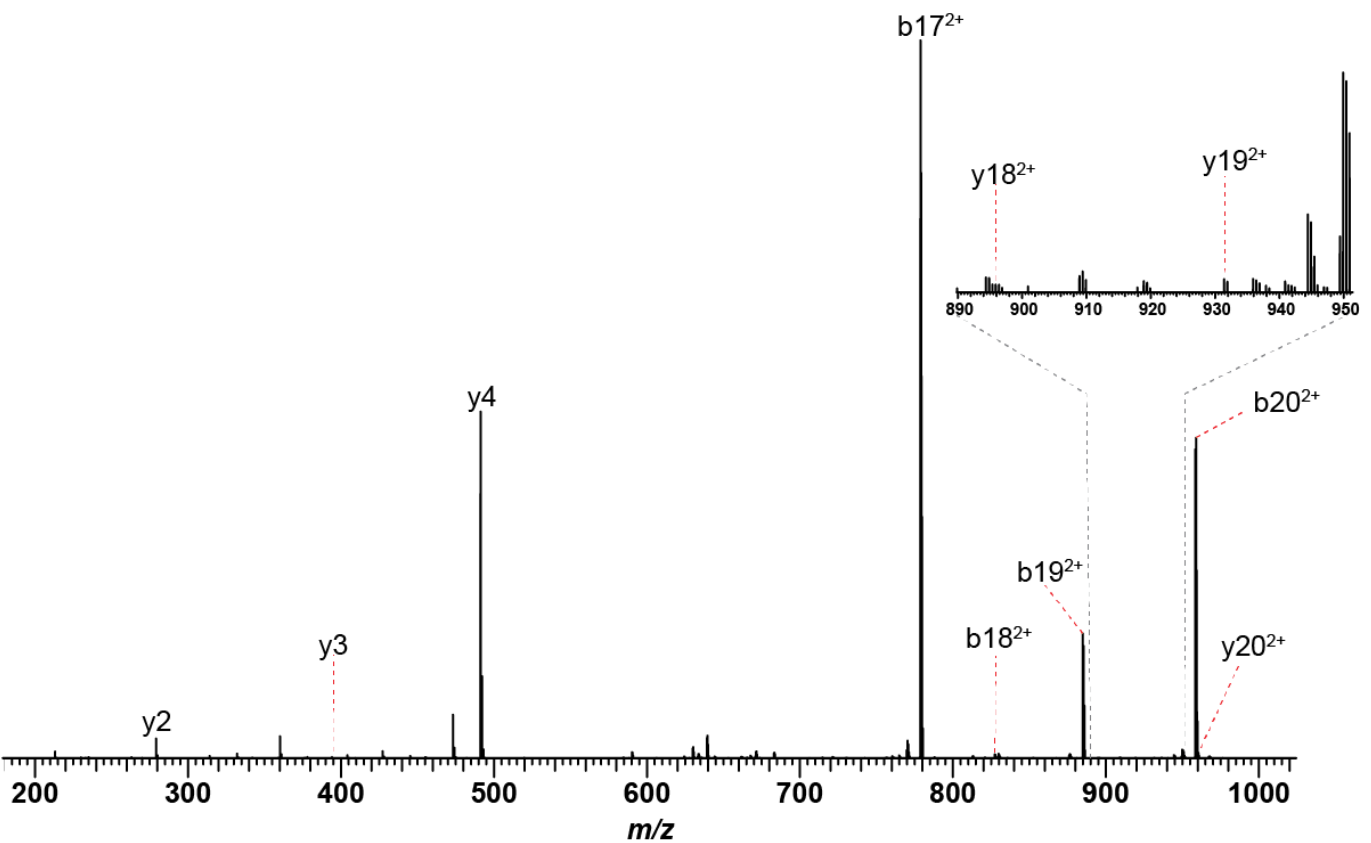

\begin{tabular}{|c|c|c|c|}
\hline Ion & Calc. Mass & Obs. Mass & Error (ppm) \\
\hline$[\mathrm{M}+3 \mathrm{H}]^{3+}$ & 682.9766 & 682.9773 & 1.0 \\
\hline b $17^{2+}$ & 778.8398 & 778.8385 & 1.7 \\
\hline b18 ${ }^{2+}$ & 827.3662 & 827.3640 & 2.8 \\
\hline b1 $19^{2+}$ & 884.8797 & 884.8779 & 2.0 \\
\hline b20 $0^{2+}$ & 958.4139 & 958.4119 & 2.1 \\
\hline y2 & 279.1703 & 279.1693 & 3.8 \\
\hline$y^{3}$ & 394.1973 & 394.1962 & 2.8 \\
\hline y4 & 491.2500 & 491.2491 & 2.0 \\
\hline$y 18^{2+}$ & 895.8845 & 895.8826 & 2.1 \\
\hline$y 19^{2+}$ & 931.4030 & 931.4010 & 2.2 \\
\hline$y 20^{2+}$ & 959.9137 & 959.9148 & 1.1 \\
\hline
\end{tabular}


B) HRMS/MS of fully methanolized doubly modified product-1 formed by ThtA-D3A variant. A. Fragmentation of doubly modified product- 1 of ThtA-D3A variant after complete methanolysis. Green denotes the substituted position. Purple indicates an Asp methyl ester. B. Annotated CID spectrum of fully methanolized doubly modified product-1 of ThtA-D3A variant. C. Table of daughter ion assignments.

A

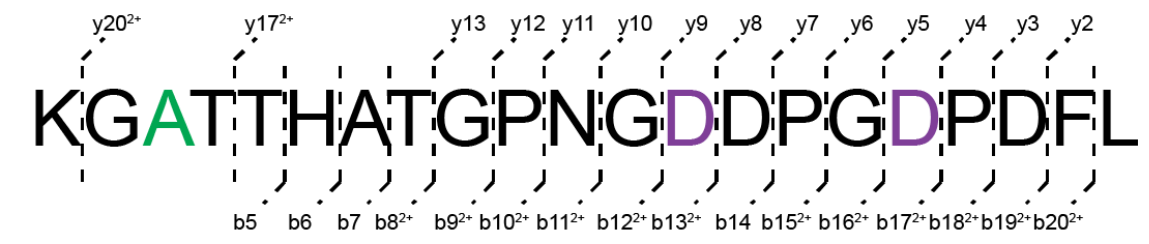

B

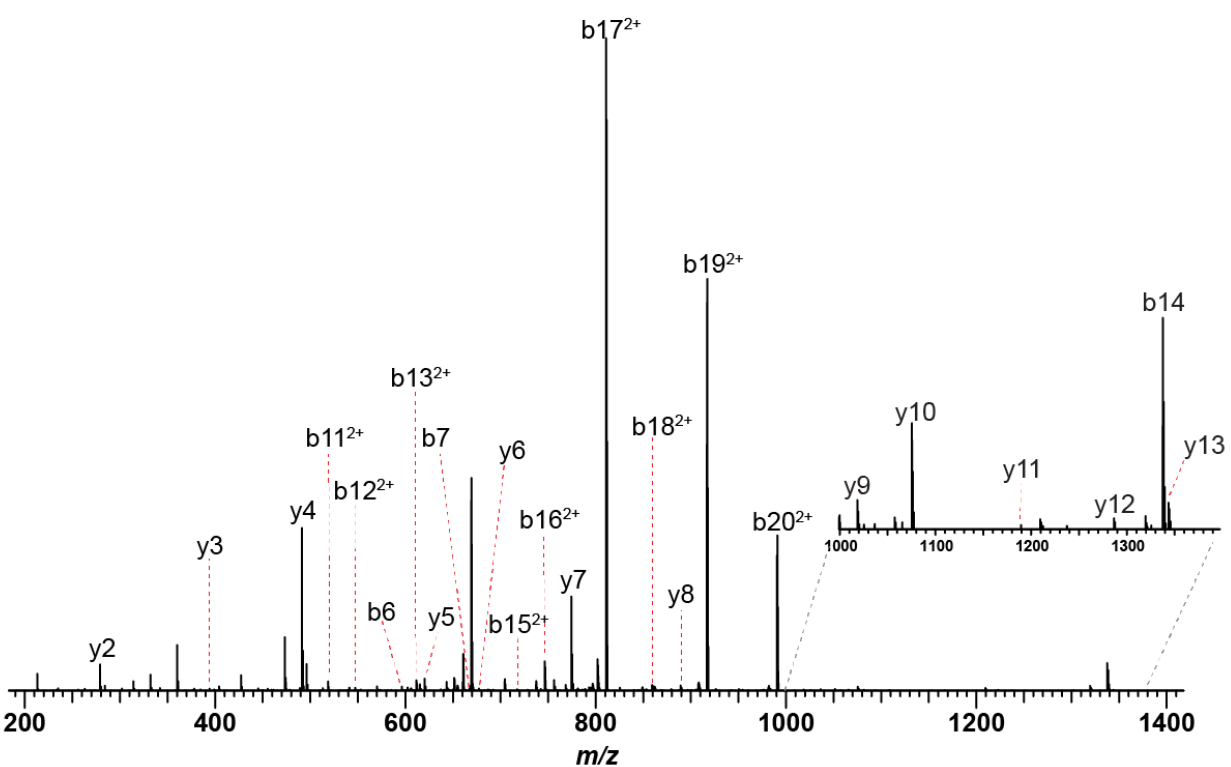

C

\begin{tabular}{|c|c|c|c|}
\hline Ion & Calc. Mass & Obs. Mass & Error (ppm) \\
\hline$[\mathrm{M}+3 \mathrm{H}]^{3+}$ & 704.3279 & 704.3271 & 1.2 \\
\hline b5 & 459.2562 & 459.2551 & 2.4 \\
\hline b6 & 596.3151 & 596.3144 & 1.2 \\
\hline b7 & 667.3522 & 667.3517 & 0.7 \\
\hline$b 8^{2+}$ & 384.7036 & 384.7025 & 2.8 \\
\hline b $9^{2+}$ & 413.2143 & 413.2132 & 2.6 \\
\hline b10 $0^{2+}$ & 461.7407 & 461.7398 & 1.9 \\
\hline b11 ${ }^{2+}$ & 518.7622 & 518.7614 & 1.4 \\
\hline b12 $2^{2+}$ & 547.2729 & 547.2729 & 0.0 \\
\hline b13 $3^{2+}$ & 611.7942 & 611.7934 & 1.3 \\
\hline b14 & 1337.6080 & 1337.6058 & 1.7 \\
\hline b15 $5^{2+}$ & 717.8340 & 717.8323 & 2.4 \\
\hline b16 $6^{2+}$ & 746.3448 & 746.3433 & 2.0 \\
\hline b1 $7^{2+}$ & 810.8661 & 810.8637 & 2.9 \\
\hline b18 ${ }^{2+}$ & 859.3924 & 859.3907 & 2.0 \\
\hline b19 ${ }^{2+}$ & 916.9059 & 916.9043 & 1.8 \\
\hline
\end{tabular}

\begin{tabular}{cccc}
\hline lon & Calc. Mass & Obs. Mass & Error (ppm) \\
\hline b20 $^{2+}$ & 990.4401 & 990.4383 & 1.8 \\
\hline y2 & 279.1703 & 279.1693 & 3.8 \\
\hline y3 & 394.1973 & 394.1962 & 2.6 \\
y4 & 491.2500 & 491.2491 & 1.9 \\
y5 & 620.2926 & 620.2918 & 1.4 \\
y6 & 677.3141 & 677.3122 & 2.8 \\
y7 & 774.3669 & 774.3642 & 3.4 \\
y8 & 889.3938 & 889.3924 & 1.6 \\
\hline y9 & 1018.4364 & 1018.4345 & 1.9 \\
\hline y10 & 1075.4579 & 1075.4558 & 1.9 \\
\hline y11 & 1189.5008 & 1189.5009 & 0.1 \\
y12 & 1286.5535 & 1286.5510 & 1.9 \\
\hline y13 & 1343.5750 & 1343.5730 & 1.5 \\
\hline y17 ${ }^{2+}$ & 877.3868 & 877.3863 & 0.6 \\
y20 $^{2+}$ & 991.9400 & 991.9421 & 2.2 \\
\hline
\end{tabular}


C) HRMS/MS of partially methanolized doubly modified product-1 formed by ThtA-D3A variant. A. Fragmentation of partially methanolized doubly modified product-1 of ThtA-D3A variant. Observed $b$ - and $y$-ions are annotated. Green denotes the substituted position. Purple indicates an Asp methyl ester. The black bracket connecting Thr and Asp indicates the site of macrolactone formation. B. Annotated CID spectrum of partially methanolized doubly modified product-1 of ThtA-D3A variant. C. Table of daughter ion assignments.

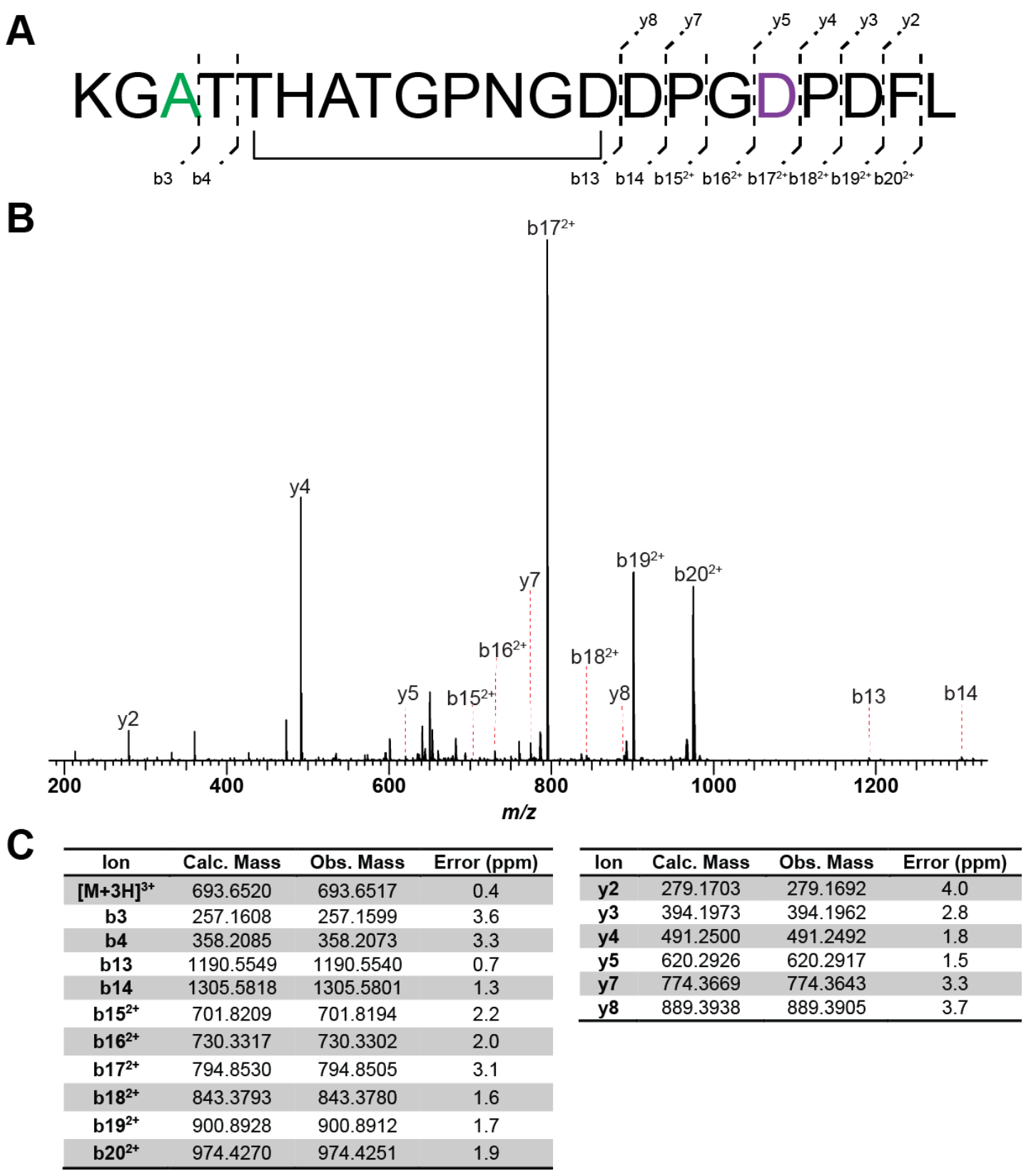


D) HRMS/MS of doubly modified product-2 formed by ThtA-D3A variant. A. Fragmentation of doubly modified product-2 of ThtA-D3A variant. Observed b- and y-ions are annotated. Green denotes the substituted position. Black brackets connecting Thr and Asp indicate sites of macrolactone formation. B. Annotated CID spectrum of doubly modified product-2 of ThtA-D3A variant. C. Table of daughter ion assignments.

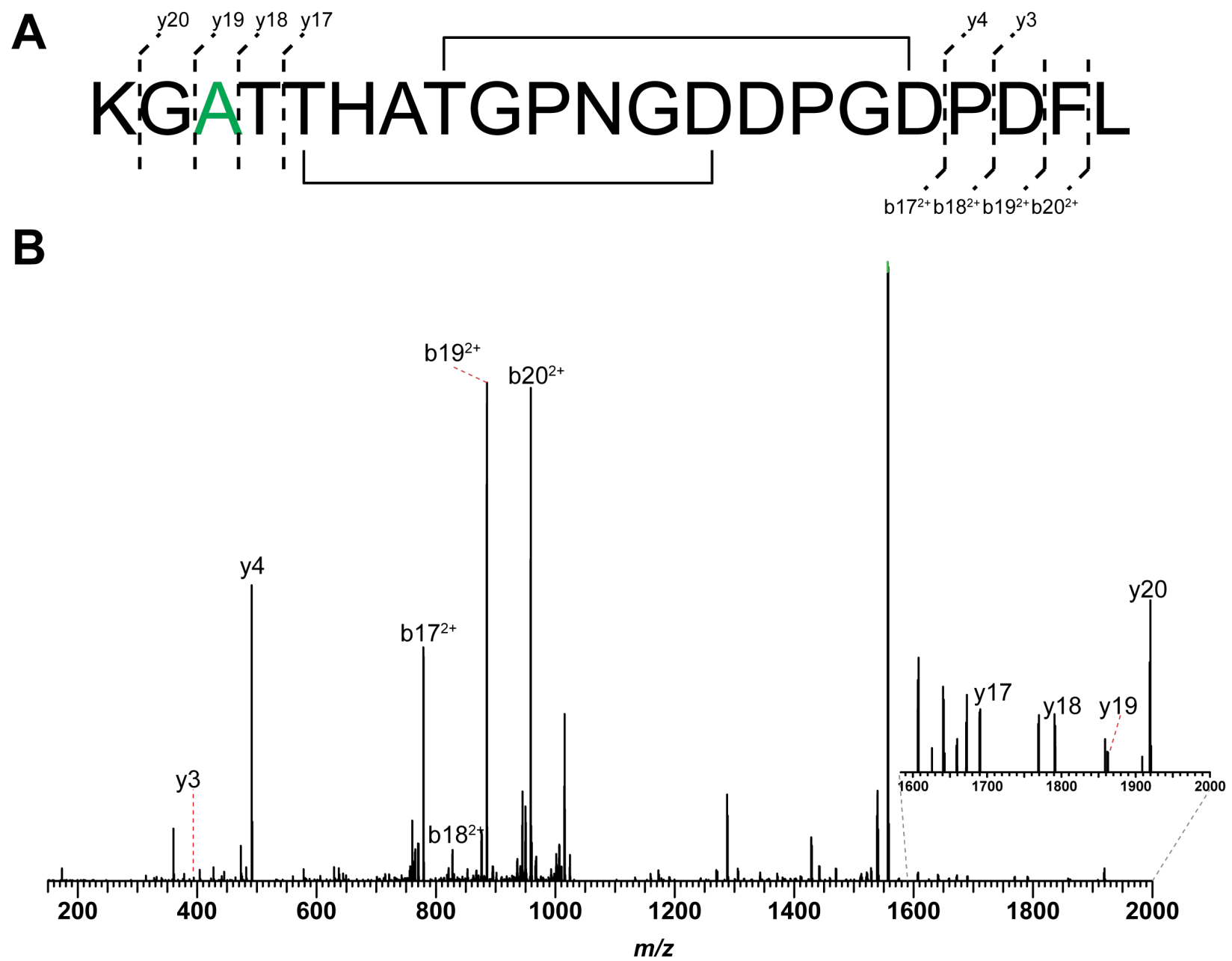

C

\begin{tabular}{cccc}
\hline Ion & Calc. Mass & Obs. Mass & Error $(\mathbf{p p m})$ \\
\hline$[\mathbf{M + 2 H}]^{2+}$ & 1023.9612 & 1023.9602 & 1.0 \\
\hline b17 $^{2+}$ & 778.8398 & 778.8385 & 1.7 \\
b18 $^{2+}$ & 827.3662 & 827.3653 & 1.1 \\
b19 $^{2+}$ & 884.8797 & 884.8781 & 1.8 \\
b20 $^{2+}$ & 958.4139 & 958.4123 & 1.7 \\
y3 & 394.1973 & 394.1969 & 0.9 \\
$\mathbf{y 4}$ & 491.2500 & 491.2493 & 1.4 \\
$\mathbf{y 1 7}$ & 1689.7140 & 1689.7106 & 2.0 \\
$\mathbf{y 1 8}$ & 1790.7616 & 1790.7589 & 1.6 \\
$\mathbf{y 1 9}$ & 1861.7988 & 1861.7937 & 2.7 \\
$\mathbf{y 2 0}$ & 1918.8202 & 1918.8173 & 1.5 \\
\hline
\end{tabular}


E) HRMS/MS of fully methanolized doubly modified product-2 formed by ThtA-D3A variant. A. Fragmentation of doubly modified product-2 of ThtA-D3A variant after complete methanolysis. Green denotes the substituted position. Purple indicates an Asp methyl ester. Observed b- and $y$-ions are annotated. B. Annotated CID spectrum of fully methanolized doubly modified product2 of ThtA-D3A variant. C. Table of daughter ion assignments.

A

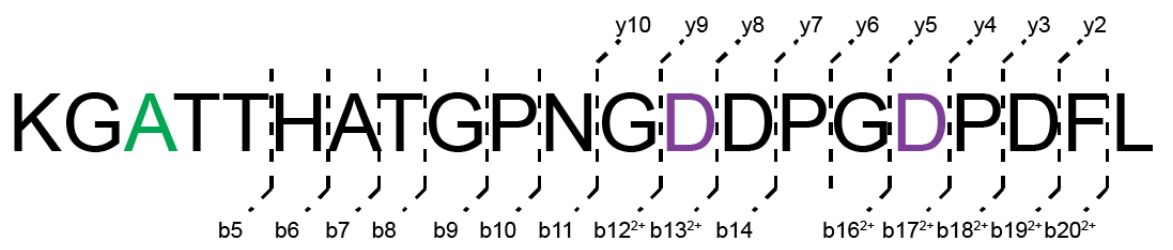

B

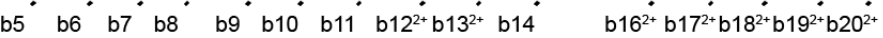

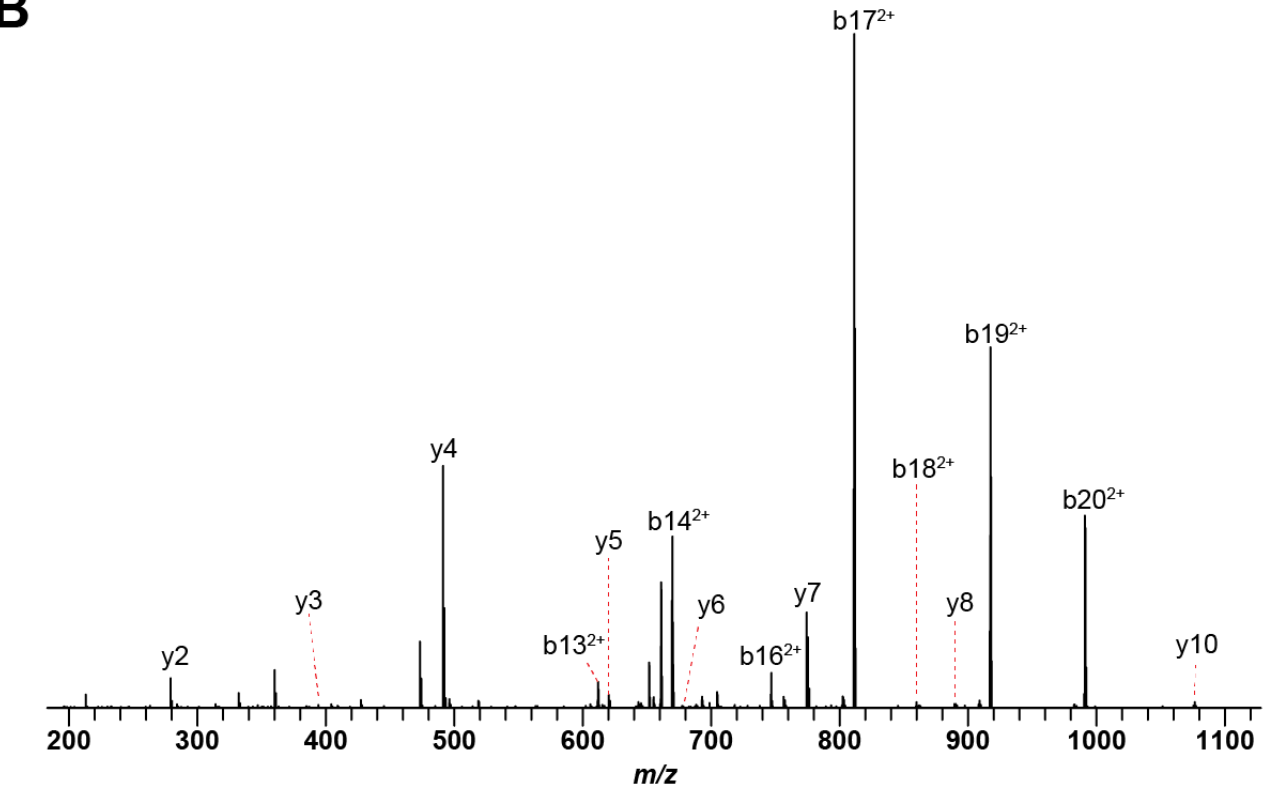

C

\begin{tabular}{|c|c|c|c|}
\hline Ion & Calc. Mass & Obs. Mass & Error (ppm) \\
\hline$[\mathrm{M}+3 \mathrm{H}]^{3+}$ & 704.3279 & 704.3269 & 1.4 \\
\hline b5 & 459.2562 & 459.2558 & 0.8 \\
\hline b6 & 596.3151 & 596.3144 & 1.1 \\
\hline b7 & 667.3522 & 667.3512 & 1.5 \\
\hline b8 & 768.3999 & 768.3990 & 1.2 \\
\hline b9 & 825.4213 & 825.4199 & 1.8 \\
\hline b10 & 922.4741 & 922.4730 & 1.2 \\
\hline b11 & 1036.5170 & 1036.5150 & 1.9 \\
\hline b12 $2^{2+}$ & 547.2729 & 547.2719 & 1.8 \\
\hline b13 ${ }^{2+}$ & 611.7942 & 611.7934 & 1.3 \\
\hline b14 ${ }^{2+}$ & 669.3077 & 669.3069 & 1.2 \\
\hline b16 ${ }^{2+}$ & 746.3448 & 746.3439 & 1.2 \\
\hline b1 $17^{2+}$ & 810.8661 & 810.8651 & 1.1 \\
\hline b18 ${ }^{2+}$ & 859.3924 & 859.3912 & 1.4 \\
\hline b192+ & 916.9059 & 916.9048 & 1.3 \\
\hline
\end{tabular}

\begin{tabular}{cccc}
\hline Ion & Calc. Mass & Obs. Mass & Error $(\mathbf{p p m})$ \\
\hline b20 $^{2+}$ & 990.4401 & 990.4388 & 1.3 \\
\hline y2 & 279.1703 & 279.1698 & 1.8 \\
y3 & 394.1973 & 394.1968 & 1.3 \\
y4 & 491.2500 & 491.2493 & 1.6 \\
y5 & 620.2926 & 620.2917 & 1.5 \\
y6 & 677.3141 & 677.3129 & 1.7 \\
y7 & 774.3669 & 774.3658 & 1.4 \\
y8 & 889.3938 & 889.3927 & 1.2 \\
y9 & 1018.4364 & 1018.4341 & 2.3 \\
\hline y10 & 1075.4579 & 1075.4562 & 1.6 \\
\hline
\end{tabular}


F) HRMS/MS of partially methanolized doubly modified product-2 formed by ThtA-D3A variant. A. Fragmentation of partially methanolized, doubly modified product-2 of ThtA-D3A variant. Observed $b$ - and $y$-ions are annotated. Green denotes the substituted position. Purple indicates an Asp methyl ester. The black bracket connecting Thr and Asp indicates the site of macrolactone formation. B. Annotated CID spectrum of partially methanolized doubly modified product-2 of ThtA-D3A variant. C. Table of daughter ion assignments.

A

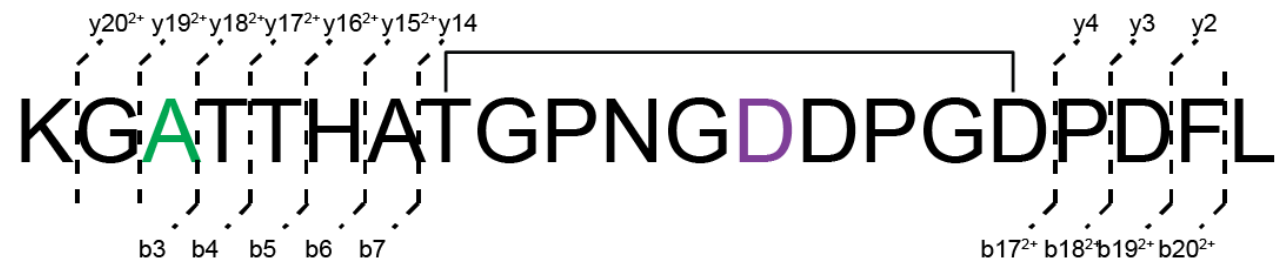

B

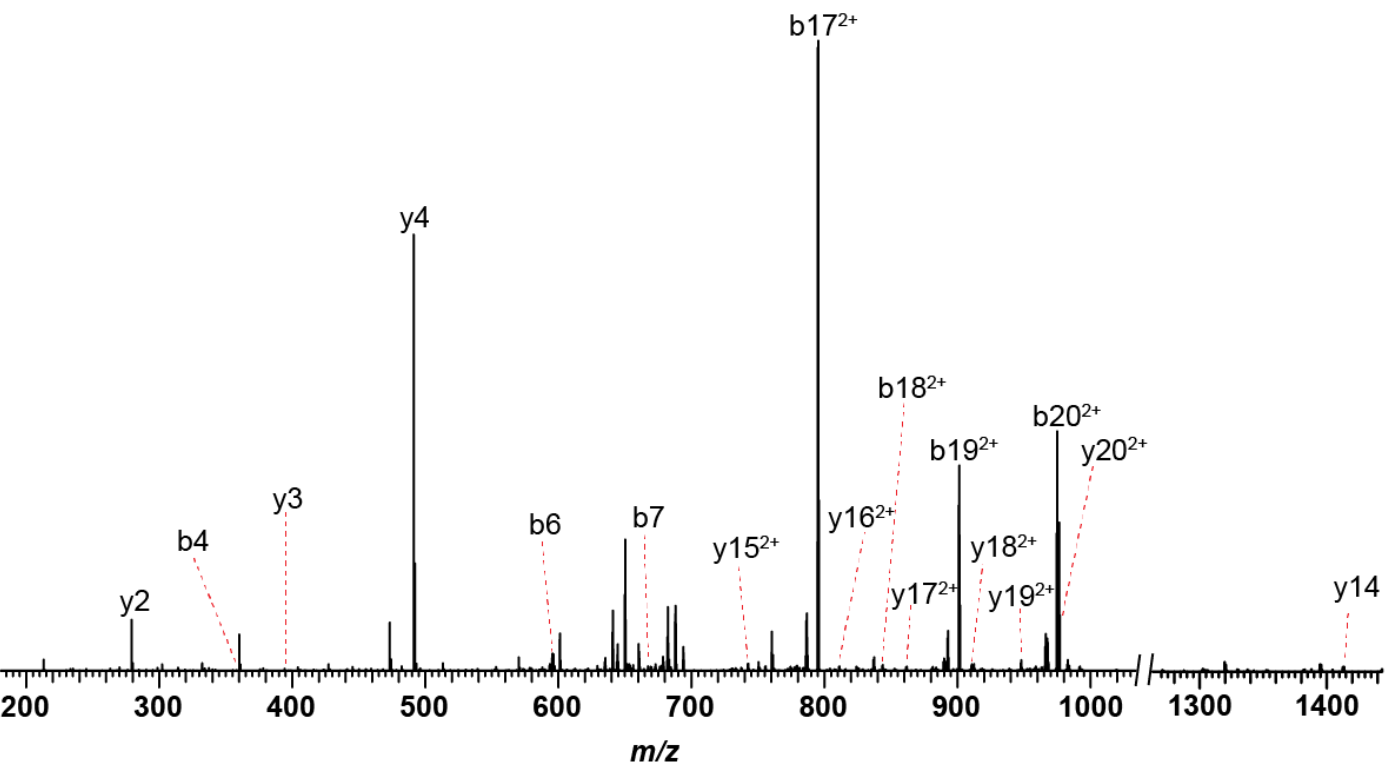

$\mathrm{c}$

\begin{tabular}{|c|c|c|c|}
\hline Ion & Calc. Mass & Obs. Mass & Error (ppm) \\
\hline$[\mathrm{M}+3 \mathrm{H}]^{3+}$ & 693.6520 & 693.6515 & 0.7 \\
\hline b3 & 257.1608 & 257.1606 & 1.0 \\
\hline b4 & 358.2085 & 358.2081 & 1.2 \\
\hline b5 & 459.2562 & 459.2557 & 1.1 \\
\hline b6 & 596.3151 & 596.3143 & 1.3 \\
\hline b7 & 667.3522 & 667.3513 & 1.3 \\
\hline b1 $17^{2+}$ & 794.8530 & 794.8518 & 1.4 \\
\hline b18 ${ }^{2+}$ & 843.3793 & 843.3786 & 0.8 \\
\hline b19 ${ }^{2+}$ & 900.8928 & 900.8914 & 1.5 \\
\hline b20 ${ }^{2+}$ & 974.4270 & 974.4259 & 1.2 \\
\hline
\end{tabular}

\begin{tabular}{cccc}
\hline lon & Calc. Mass & Obs. Mass & Error $(\mathbf{p p m})$ \\
\hline $\mathbf{y 2}$ & 279.1703 & 279.1699 & 1.5 \\
$\mathbf{y 3}$ & 394.1973 & 394.1968 & 1.1 \\
$\mathbf{y 4}$ & 491.2500 & 491.2494 & 1.3 \\
$\mathbf{y 1 4}$ & 1412.5965 & 1412.5939 & 1.8 \\
$\mathbf{y} 15^{2+}$ & 742.3204 & 742.3191 & 1.7 \\
$\mathbf{y 1 6}^{2+}$ & 810.8499 & 810.8488 & 1.3 \\
$\mathbf{y 1 7}^{2+}$ & 861.3737 & 861.3723 & 1.6 \\
$\mathbf{y} \mathbf{1 8}^{2+}$ & 911.8976 & 911.8963 & 1.4 \\
$\mathbf{y 1 9}^{2+}$ & 947.4161 & 947.4148 & 1.4 \\
$\mathbf{y 2 0 ^ { 2 + }}$ & 975.9269 & 975.9248 & 2.1 \\
\hline
\end{tabular}


G) HRMS/MS of singly modified product formed by ThtA-D3A variant. A. Fragmentation of singly modified product formed upon co-expression of ThtA-D3A variant with ThtB. Observed band $y$-ions are annotated. Green denotes the substituted position. The black bracket connecting B. Annotated CID spectrum of singly modified product. C. Table of daughter ion assignments.

A

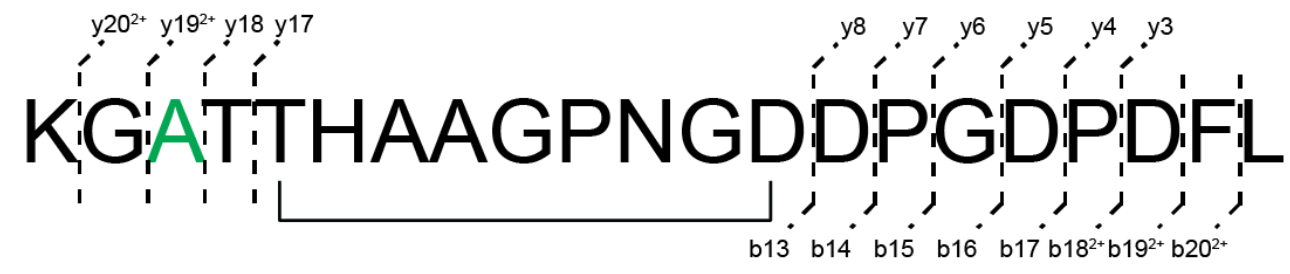

B

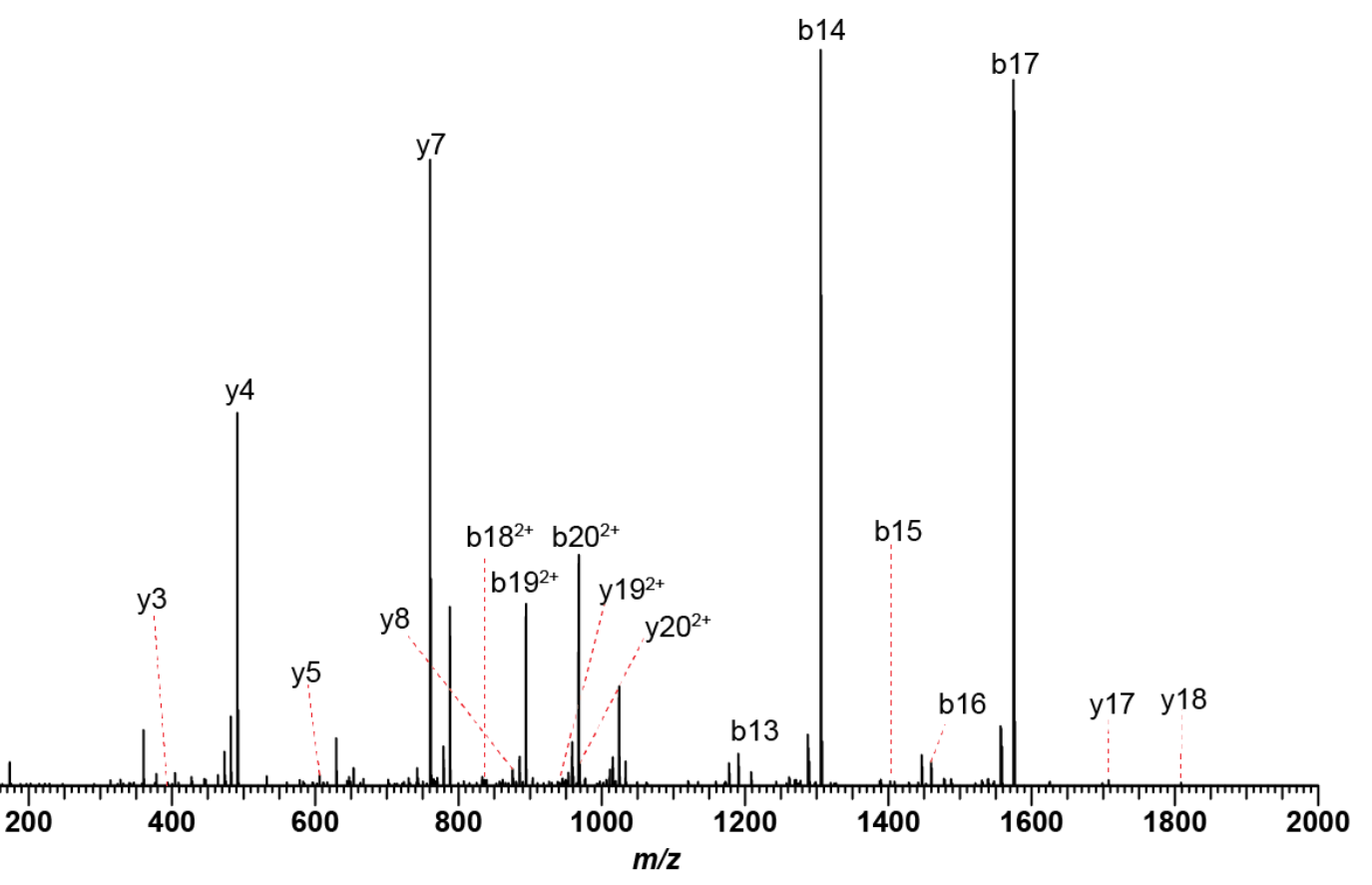

\begin{tabular}{|c|c|c|c|}
\hline Ion & Calc. Mass & Obs. Mass & Error (ppm) \\
\hline$[\mathrm{M}+2 \mathrm{H}]^{2+}$ & 1032.9665 & 1032.9655 & 0.9 \\
\hline b13 & 1190.5549 & 1190.5524 & 2.1 \\
\hline b14 & 1305.5818 & 1305.5795 & 1.8 \\
\hline b15 & 1402.6346 & 1402.6361 & 1.1 \\
\hline b16 & 1459.6561 & 1459.6543 & 1.2 \\
\hline b17 & 1574.6830 & 1574.6805 & 1.6 \\
\hline b18 ${ }^{2+}$ & 836.3715 & 836.3706 & 1.1 \\
\hline b1 $19^{2+}$ & 893.8850 & 893.8837 & 1.4 \\
\hline b20 ${ }^{2+}$ & 967.4192 & 967.4178 & 1.5 \\
\hline y3 & 394.1973 & 394.1967 & 1.5 \\
\hline
\end{tabular}

\begin{tabular}{cccc}
\hline lon & Calc. Mass & Obs. Mass & Error $(\mathbf{p p m})$ \\
\hline y4 & 491.2500 & 491.2493 & 1.6 \\
y5 & 606.2770 & 606.2762 & 1.3 \\
y6 & 663.2984 & 663.2979 & 0.8 \\
y7 & 760.3512 & 760.3500 & 1.6 \\
y8 & 875.3781 & 875.3770 & 1.3 \\
y17 & 1707.7245 & 1707.7208 & 2.2 \\
y18 & 1808.7722 & 1808.7716 & 0.4 \\
\hline y19 $^{2+}$ & 940.4083 & 940.4046 & 4.0 \\
y20 $^{2+}$ & 968.9190 & 968.9172 & 1.9 \\
\hline
\end{tabular}


H) MS/MS of methanolized singly modified product formed by ThtA-D3A variant. A. Fragmentation of methanolized singly modified product formed upon co-expression of ThtA-D3A variant with ThtB. Observed b- and y-ions are annotated. Green denotes the substituted position. Purple denotes an Asp methyl ester. B. Annotated CID spectrum of the fully methanolized singly modified product. C. Table of daughter ion assignments.

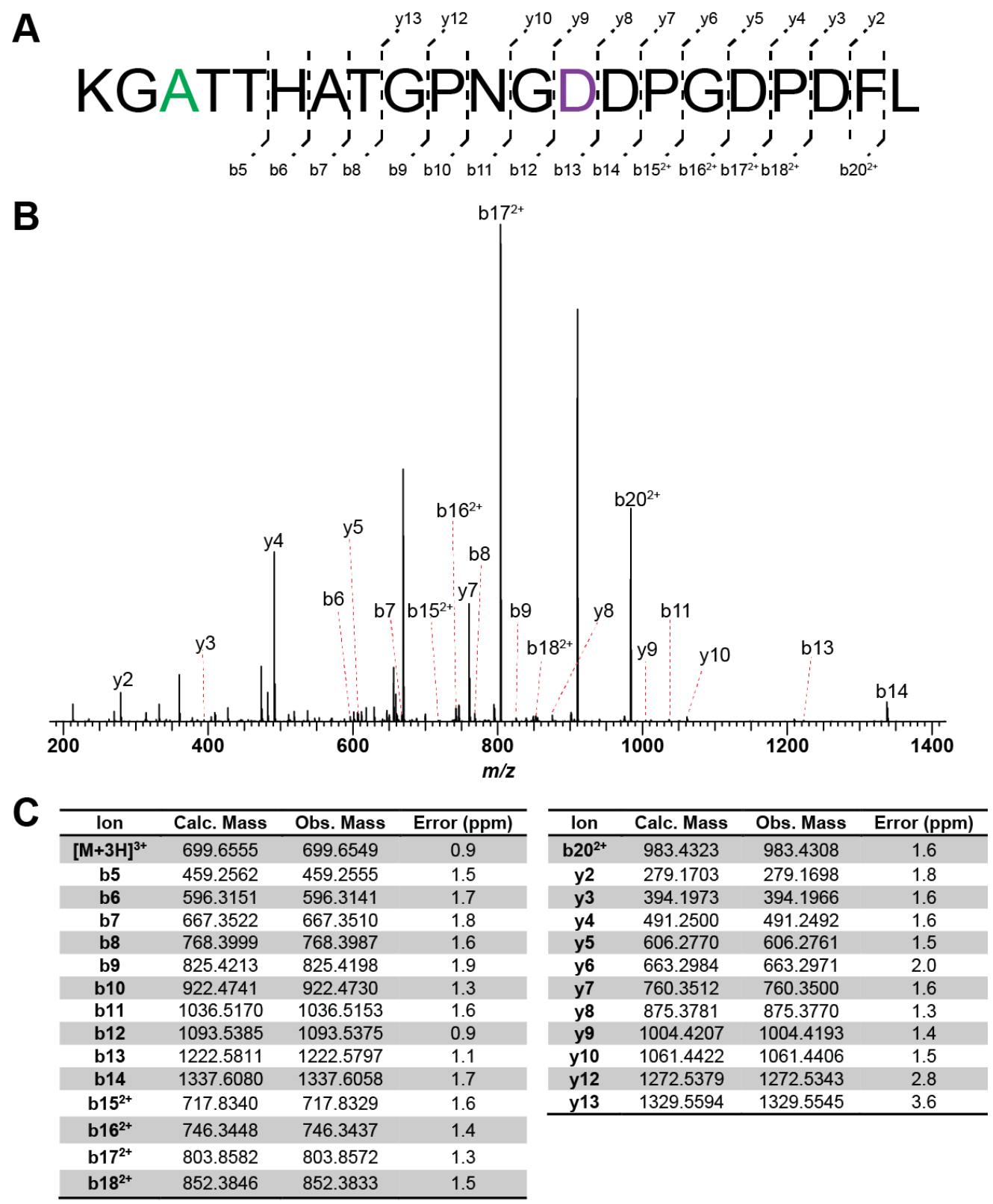


Figure S23. ThtA-T4A variant- modified products: HRMS/MS.

A) HRMS/MS of doubly modified product-1 formed by T4A variant. A. Fragmentation of doubly modified product-1 of ThtA-T4A variant. Observed b- and y-ions are annotated. Green denotes the substituted position. Black brackets connecting Thr and Asp indicate sites of macrolactone formation. B. Annotated CID spectrum of doubly modified product-1 of ThtA-T4A variant. C. Table of daughter ion assignments.

A

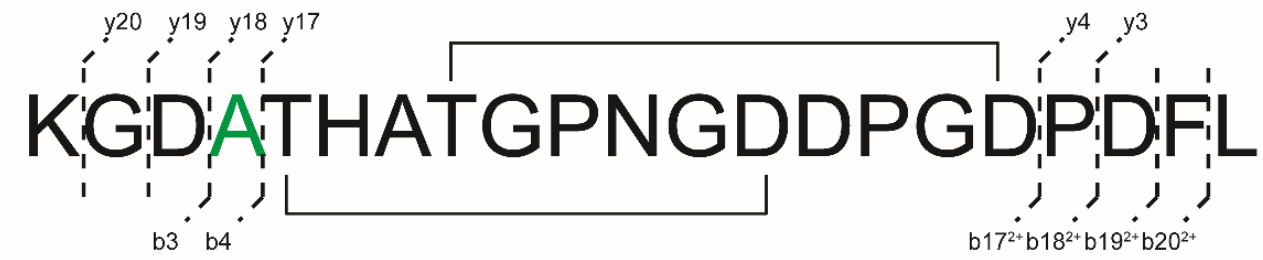

B

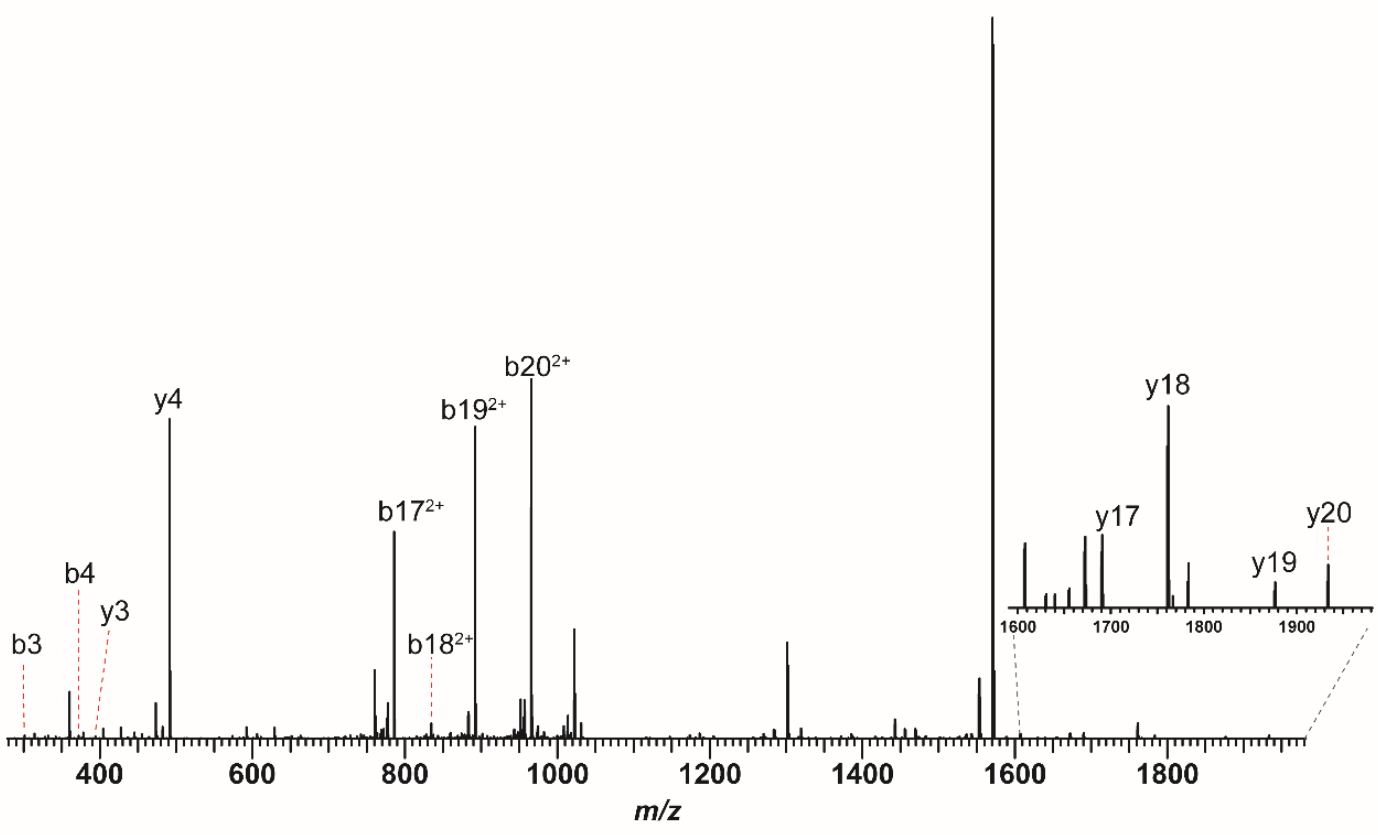

C

\begin{tabular}{cccc}
\hline Ion & Calc. Mass & Obs. Mass & Error $(\mathbf{p p m})$ \\
\hline $\mathbf{[ M + 2 H}^{2+}$ & 1030.9509 & 1030.9500 & 0.9 \\
$\mathbf{b 3}$ & 301.1507 & 301.1502 & 1.4 \\
$\mathbf{b 4}$ & 372.1878 & 372.1875 & 0.8 \\
b17 $^{2+}$ & 785.8295 & 785.8284 & 1.4 \\
b18 $^{2+}$ & 834.3559 & 834.3555 & 0.4 \\
b19 $^{2+}$ & 891.8693 & 891.8682 & 1.3 \\
b20 $^{2+}$ & 965.4035 & 965.4024 & 1.2 \\
$\mathbf{y 3}$ & 394.1973 & 394.1967 & 1.3 \\
$\mathbf{y 4}$ & 491.2500 & 491.2493 & 1.5 \\
$\mathbf{y 1 7}$ & 1689.7140 & 1689.7103 & 2.1 \\
\hline
\end{tabular}

\begin{tabular}{cccc}
\hline Ion & Calc. Mass & Obs. Mass & Error $(\mathbf{p p m})$ \\
\hline y18 & 1760.7511 & 1760.7471 & 2.3 \\
y19 & 1875.7780 & 1875.7718 & 3.3 \\
y20 & 1932.7995 & 1932.7959 & 1.9 \\
\hline
\end{tabular}


B) HRMS/MS of fully methanolized doubly modified product-1 formed by ThtA-T4A variant. A. Fragmentation of doubly modified product- 1 of ThtA-T4A variant after complete methanolysis. Green denotes the substituted position. Purple indicates an Asp methyl ester. Observed b- and $y$-ions are annotated. B. Annotated CID spectrum of fully methanolized doubly modified product1 of ThtA-T4A variant. C. Table of daughter ion assignments.

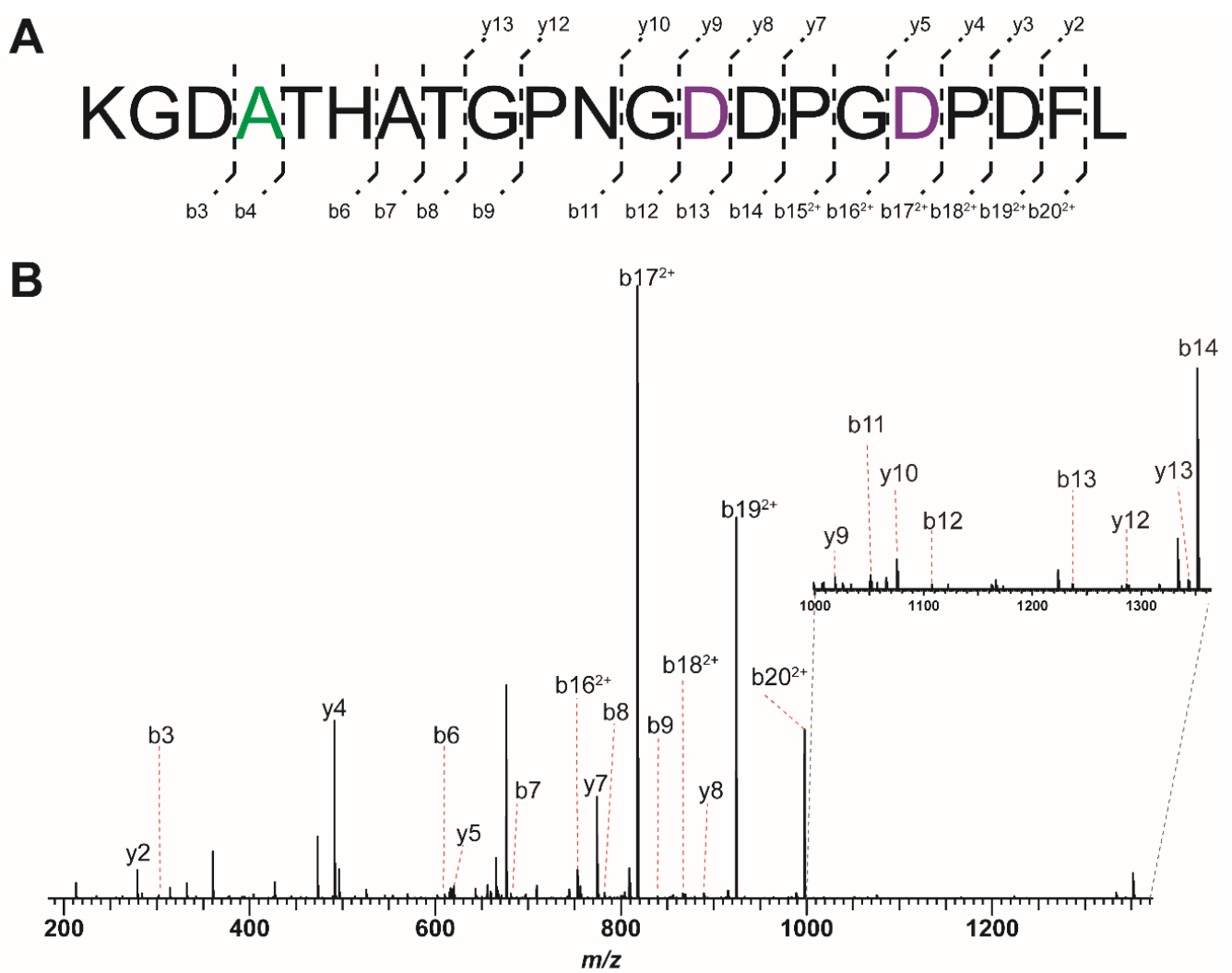

\begin{tabular}{cccc}
$\mathbf{C}$ & \multicolumn{4}{c}{} \\
\cline { 2 - 5 } Ion & Calc. Mass & Obs. Mass & Error (ppm) \\
\hline${\text { [M+3H }]^{3+}}^{3}$ & 708.9871 & 708.9865 & 0.9 \\
b3 & 301.1507 & 301.1501 & 2.0 \\
b4 & 372.1878 & 372.1871 & 1.8 \\
b6 & 610.2944 & 610.2932 & 1.9 \\
\hline b7 & 681.3315 & 681.3302 & 1.8 \\
b8 & 782.3791 & 782.3777 & 1.8 \\
\hline b9 & 839.4006 & 839.3990 & 1.9 \\
\hline b11 & 1050.4963 & 1050.4938 & 2.4 \\
\hline b12 & 1107.5178 & 1107.5174 & 0.3 \\
\hline b13 & 1236.5604 & 1236.5587 & 1.3 \\
\hline b14 & 1351.5873 & 1351.5844 & 2.1 \\
\hline b15 ${ }^{2+}$ & 724.8237 & 724.8227 & 1.3 \\
\hline b16 $^{2+}$ & 753.3344 & 753.3330 & 1.8 \\
\hline b17 $^{2+}$ & 817.8557 & 817.8541 & 1.9 \\
\hline b18 $^{2+}$ & 866.3821 & 866.3806 & 1.7 \\
\hline
\end{tabular}

\begin{tabular}{cccc}
\hline Ion & Calc. Mass & Obs. Mass & Error $(\mathbf{p p m})$ \\
\hline b19 $^{2+}$ & 923.8956 & 923.8938 & 1.9 \\
b20 $^{2+}$ & 997.4298 & 997.4277 & 2.0 \\
y2 & 279.1703 & 279.1698 & 1.7 \\
y3 & 394.1973 & 394.1967 & 1.5 \\
y4 & 491.2500 & 491.2493 & 1.4 \\
y5 & 620.2926 & 620.2914 & 2.0 \\
y7 & 774.3669 & 774.3654 & 1.9 \\
y8 & 889.3938 & 889.3922 & 1.8 \\
y9 & 1018.4364 & 1018.4343 & 2.0 \\
y10 & 1075.4579 & 1075.4553 & 2.3 \\
y12 & 1286.5535 & 1286.5521 & 1.1 \\
y13 & 1343.5750 & 1343.5713 & 2.8 \\
\hline
\end{tabular}


C) HRMS/MS of partially methanolized doubly modified product-1 formed by ThtA-T4A variant. A. Fragmentation of partially methanolized doubly modified product-1 of ThtA-T4A variant. Observed $b$ - and $y$-ions are annotated. Green denotes the substituted position. Purple indicates an Asp methyl ester. The black bracket connecting Thr and Asp indicates the site of macrolactone formation. B. Annotated CID spectrum of partially methanolized doubly modified product-1 of ThtA-T4A variant. C. Table of daughter ion assignments.

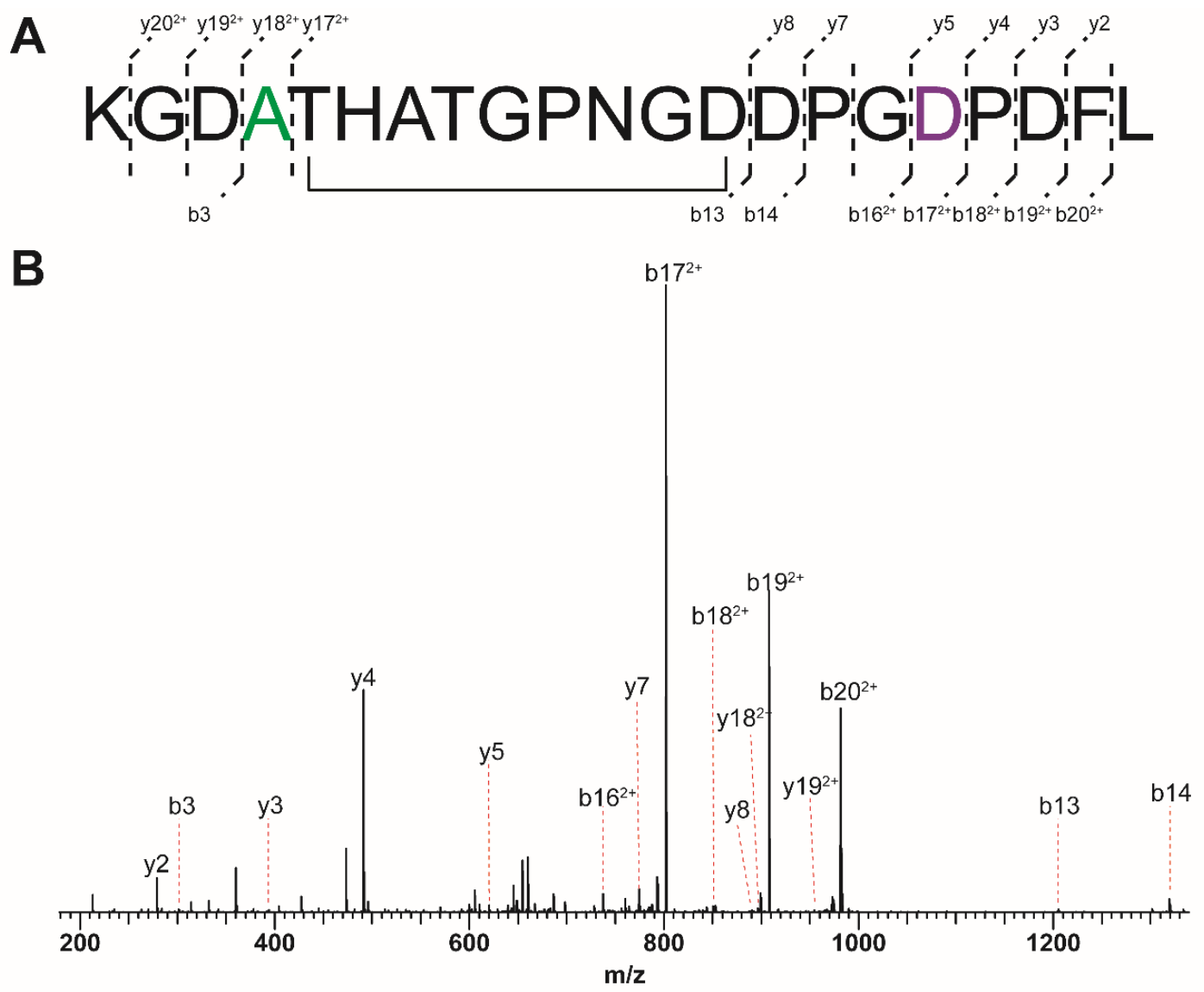

\begin{tabular}{|c|c|c|c|}
\hline Ion & Calc. Mass & Obs. Mass & Error (ppm) \\
\hline$[\mathrm{M}+3 \mathrm{H}]^{3+}$ & 698.3117 & 698.3112 & 0.7 \\
\hline b3 & 301.1507 & 301.1502 & 1.6 \\
\hline b13 & 1204.5341 & 1204.5315 & 2.2 \\
\hline b14 & 1319.5611 & 1319.5579 & 2.4 \\
\hline b16 ${ }^{2+}$ & 737.3213 & 737.3199 & 1.9 \\
\hline b1 $17^{2+}$ & 801.8426 & 801.8413 & 1.6 \\
\hline b18 ${ }^{2+}$ & 850.3690 & 850.3678 & 1.4 \\
\hline b19 ${ }^{2+}$ & 907.8824 & 907.8808 & 1.8 \\
\hline b20 ${ }^{2+}$ & 981.4166 & 981.4148 & 1.9 \\
\hline y2 & 279.1703 & 279.1699 & 1.5 \\
\hline
\end{tabular}

\begin{tabular}{cccc}
\hline Ion & Calc. Mass & Obs. Mass & Error $(\mathbf{p p m})$ \\
\hline $\mathbf{y 3}$ & 394.1973 & 394.1968 & 1.2 \\
$\mathbf{y 4}$ & 491.2500 & 491.2493 & 1.5 \\
$\mathbf{y 5}$ & 620.2926 & 620.2915 & 1.8 \\
$\mathbf{y 7}$ & 774.3669 & 774.3655 & 1.8 \\
$\mathbf{y 8}$ & 889.3938 & 889.3923 & 1.7 \\
$\mathbf{y 1 7 ^ { 2 + }}$ & 861.3737 & 861.3723 & 1.6 \\
$\mathbf{y 1 8}^{2+}$ & 896.8923 & 896.8911 & 1.3 \\
$\mathbf{y 1 9}^{2+}$ & 954.4058 & 954.4041 & 1.7 \\
$\mathbf{y 2 0}^{2+}$ & 982.9165 & 982.9139 & 2.6 \\
\hline
\end{tabular}


D) HRMS/MS of doubly modified product-2 formed by ThtA-T4A variant. A. Fragmentation of doubly modified product-2 of ThtA-T4A variant. Observed b- and y-ions are annotated. Green denotes the substituted position. Black brackets connecting Thr and Asp indicate sites of macrolactone formation. B. Annotated CID spectrum of doubly modified product-2 of ThtA-T4A variant. C. Table of daughter ion assignments.

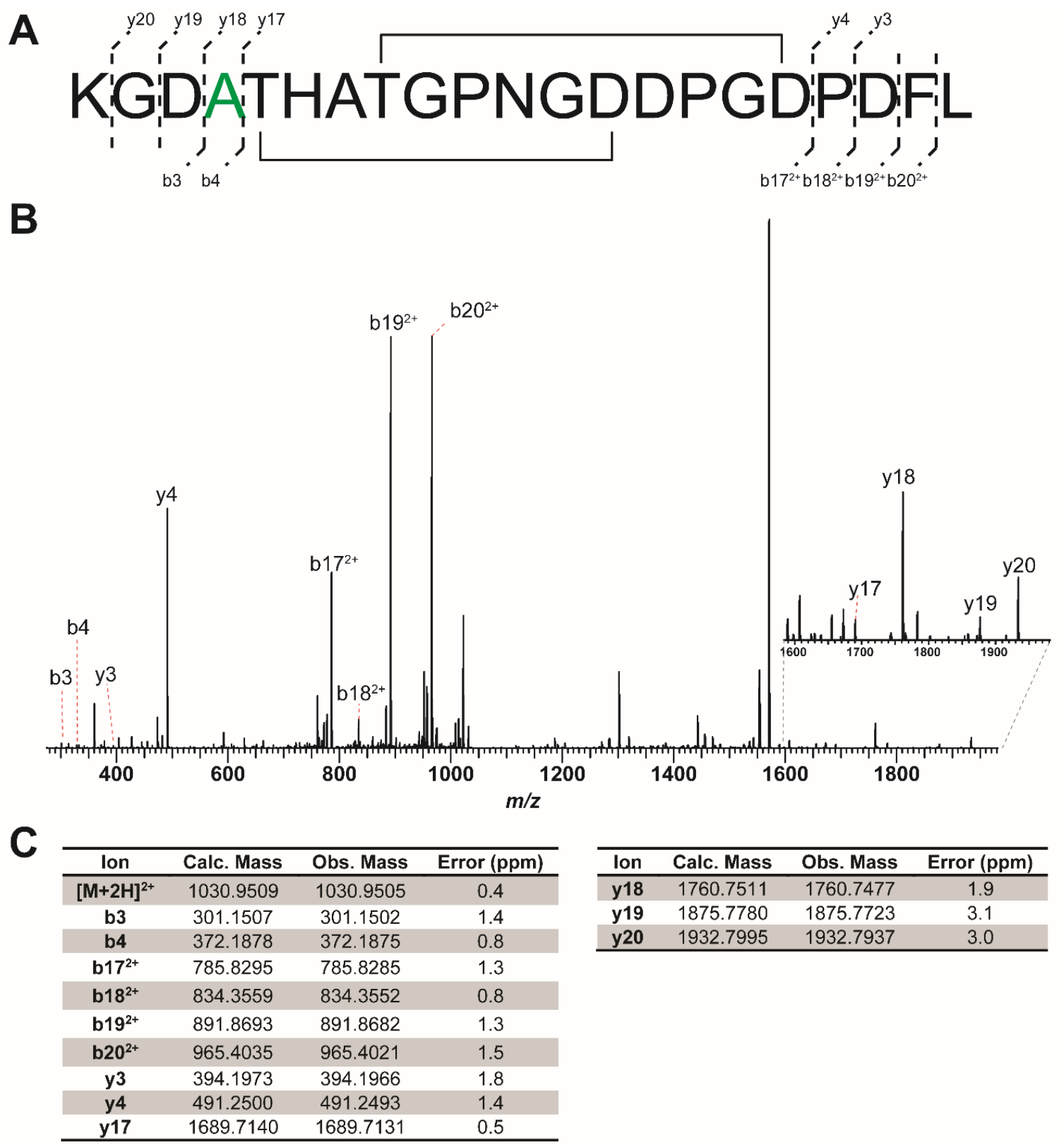


E) HRMS/MS of fully methanolized doubly modified product-2 formed by ThtA-T4A variant. A. Fragmentation of doubly modified product-2 of ThtA-T4A variant after complete methanolysis. Green denotes the substituted position. Purple indicates an Asp methyl ester. Observed b- and $y$-ions are annotated. B. Annotated CID spectrum of fully methanolized doubly modified product2 of ThtA-T4A variant. C. Table of daughter ion assignments.

A

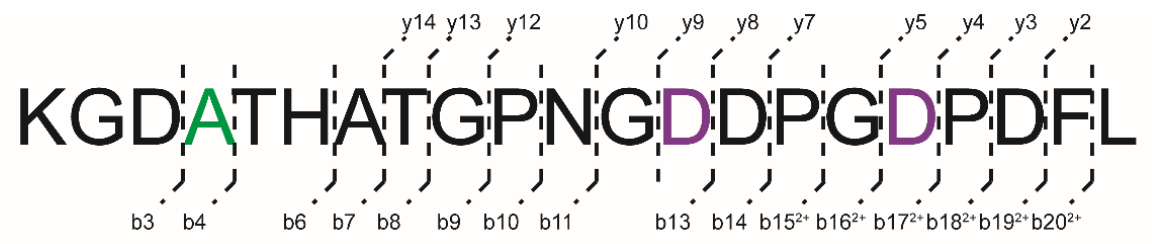

B

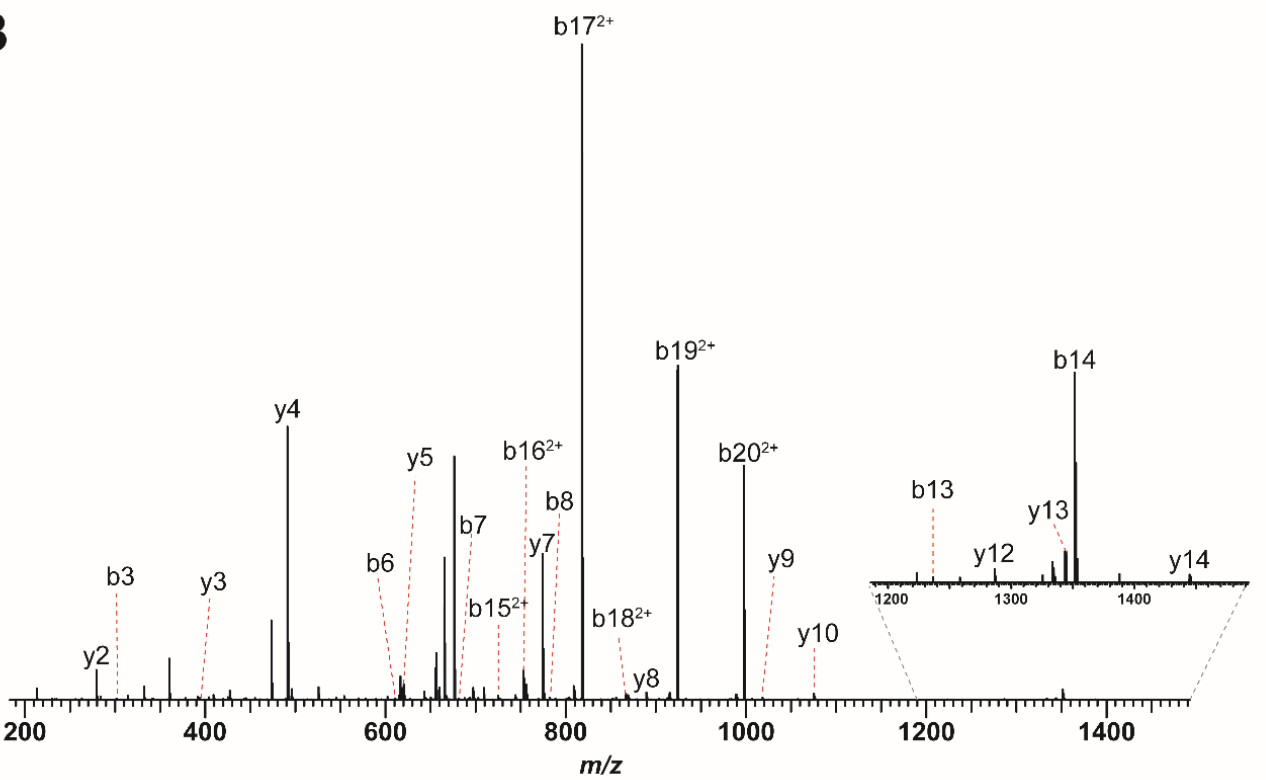

C

\begin{tabular}{cccc}
\hline Ion & Calc. Mass & Obs. Mass & Error $(\mathbf{p p m})$ \\
\hline${\text { [M+3H }]^{3+}}^{\mathbf{M}}$ & 708.9871 & 708.9866 & 0.8 \\
\hline b3 & 301.1507 & 301.1502 & 1.7 \\
b4 & 372.1878 & 372.1874 & 1.0 \\
b6 & 610.2944 & 610.2936 & 1.3 \\
b7 & 681.3315 & 681.3304 & 1.6 \\
b8 & 782.3791 & 782.3782 & 1.3 \\
b9 & 839.4006 & 839.3991 & 1.8 \\
b10 & 936.4534 & 936.4538 & 0.5 \\
b11 & 1050.4963 & 1050.4944 & 1.8 \\
b13 & 1236.5604 & 1236.5580 & 1.9 \\
\hline b14 & 1351.5873 & 1351.5848 & 1.8 \\
b15 ${ }^{2+}$ & 724.8237 & 724.8229 & 1.1 \\
b16 $^{2+}$ & 753.3344 & 753.3333 & 1.5 \\
b17 $^{2+}$ & 817.8557 & 817.8547 & 1.2 \\
b18 $^{2+}$ & 866.3821 & 866.3809 & 1.4 \\
\hline
\end{tabular}

\begin{tabular}{cccc}
\hline Ion & Calc. Mass & Obs. Mass & Error $(\mathbf{p p m})$ \\
\hline b19 $^{2+}$ & 923.8956 & 923.8941 & 1.6 \\
b20 $^{2+}$ & 997.4298 & 997.4283 & 1.4 \\
y2 $^{2}$ & 279.1703 & 279.1699 & 1.6 \\
y3 & 394.1973 & 394.1967 & 1.4 \\
y4 & 491.2500 & 491.2494 & 1.4 \\
y5 & 620.2926 & 620.2918 & 1.4 \\
y7 & 774.3669 & 774.3659 & 1.3 \\
y8 & 889.3938 & 889.3927 & 1.2 \\
\hline y9 & 1018.4364 & 1018.4346 & 1.8 \\
y10 & 1075.4579 & 1075.4561 & 1.6 \\
y12 & 1286.5535 & 1286.5515 & 1.6 \\
y13 & 1343.5750 & 1343.5738 & 0.9 \\
y14 & 1444.6227 & 1444.6195 & 2.2 \\
\hline
\end{tabular}


F) HRMS/MS of partially methanolized doubly modified product-2 formed by ThtA-T4A variant. A. Fragmentation of partially methanolized doubly modified product-2 of ThtA-T4A variant. Observed $b$ - and $y$-ions are annotated. Green denotes the substituted position. Purple indicates an Asp methyl ester. The black bracket connecting Thr and Asp indicates the site of macrolactone formation. B. Annotated CID spectrum of partially methanolized doubly modified product-2 of ThtA-T4A variant. C. Table of daughter ion assignments.

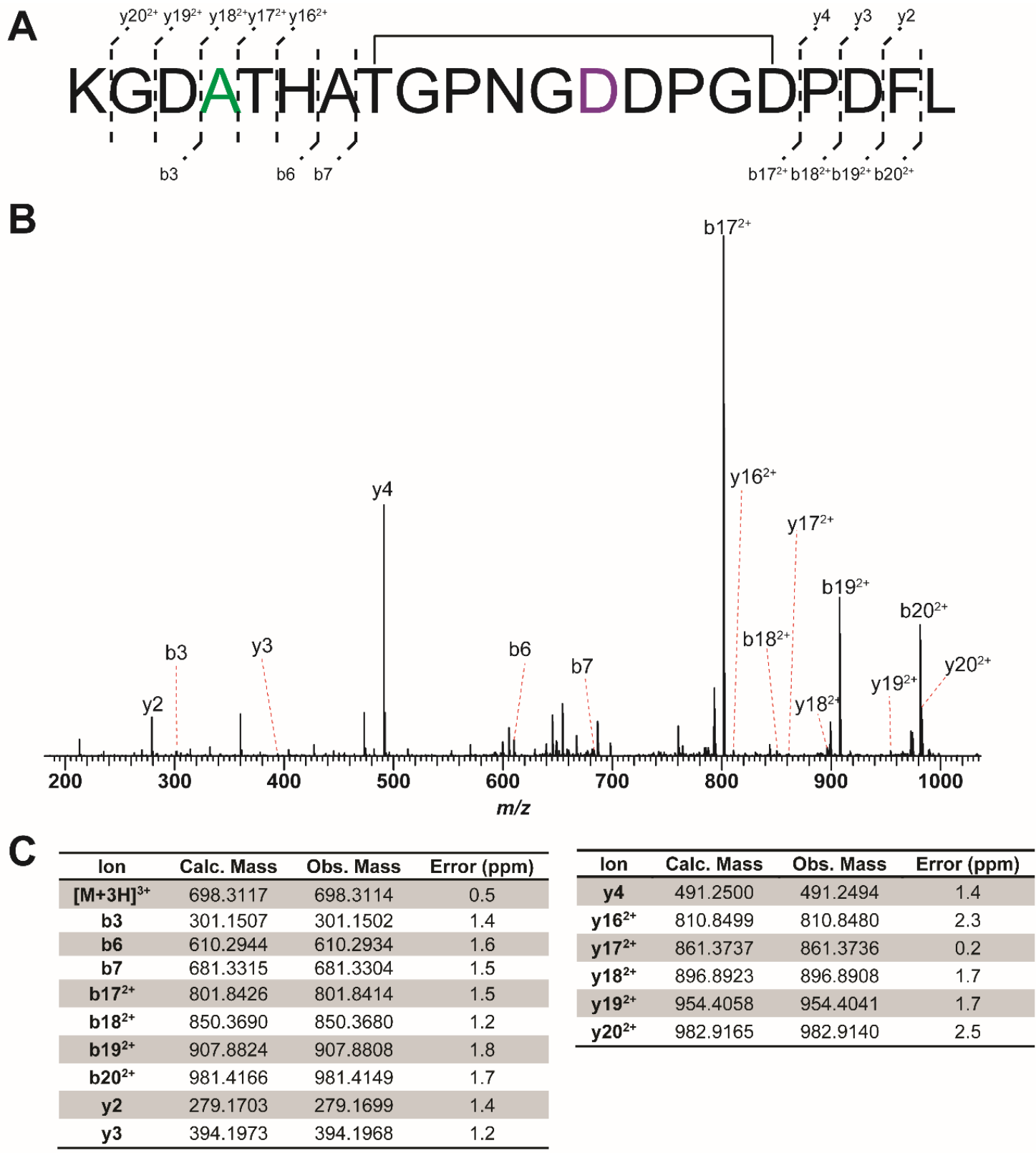


Figure S24. ThtA-D14A variant-modified products: HRMS/MS.

A) HRMS/MS of doubly modified product formed by ThtA-D14A variant. A. Fragmentation of doubly modified product of ThtA-D14A variant. Observed b- and y-ions are annotated. Green denotes the substituted position. Black brackets connecting Thr and Asp indicate sites of macrolactone formation. B. Annotated CID spectrum of doubly modified product of ThtA-D14A variant. C. Table of daughter ion assignments.

A

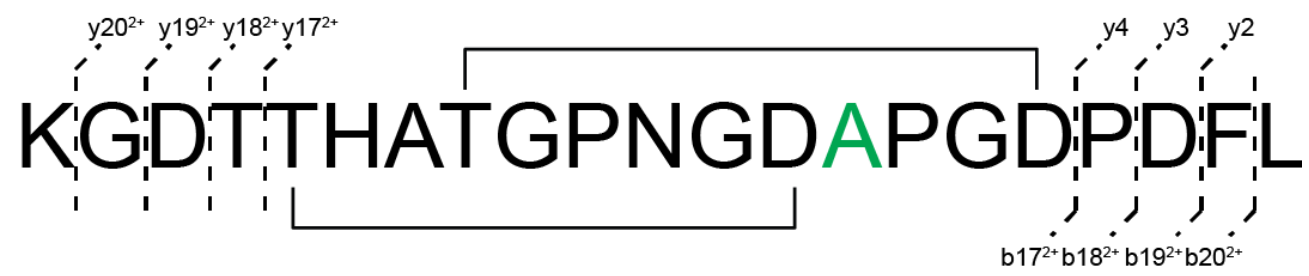

B

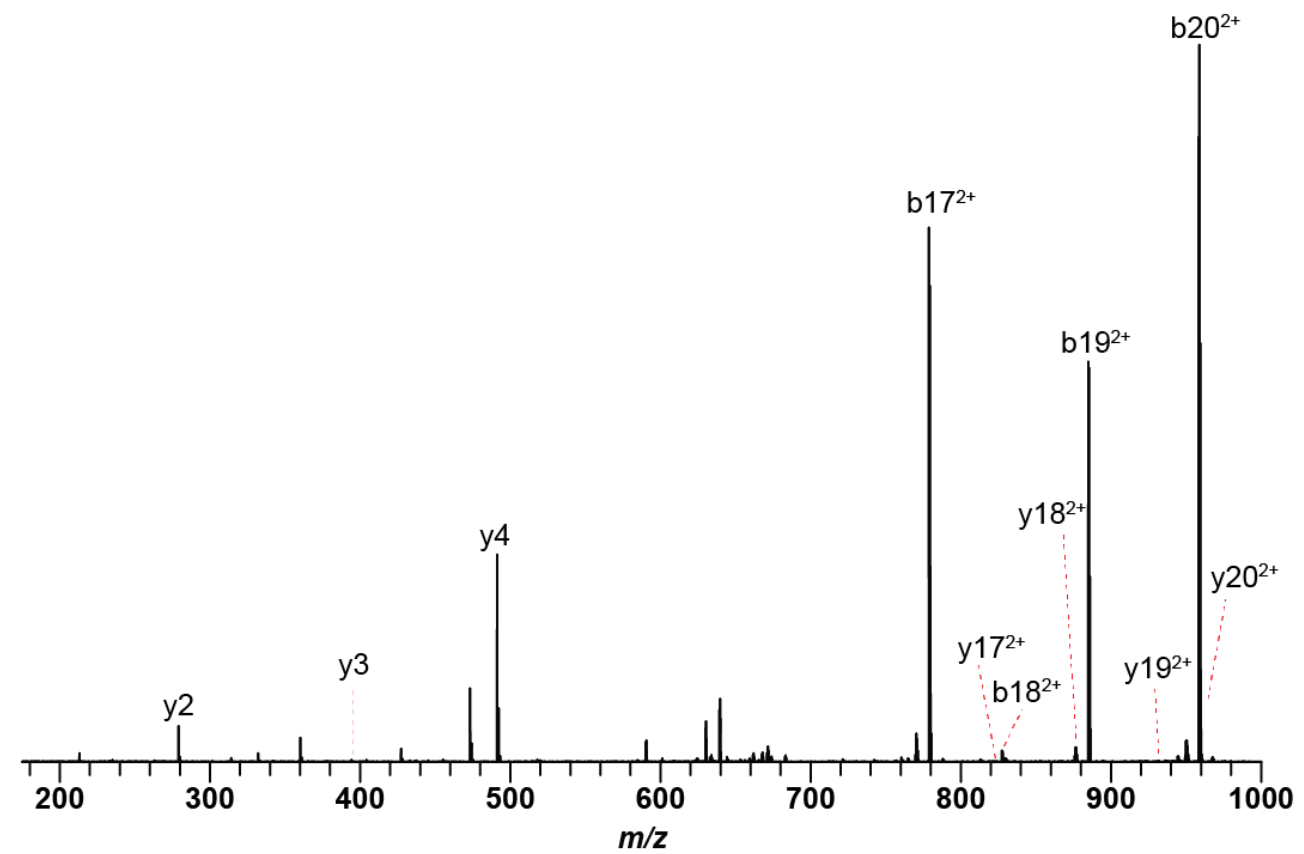

C

\begin{tabular}{|c|c|c|c|}
\hline Ion & Calc. Mass & Obs. Mass & Error (ppm) \\
\hline$[\mathrm{M}+3 \mathrm{H}]^{3+}$ & 682.9766 & 682.9764 & 0.3 \\
\hline b1 $17^{2+}$ & 778.8398 & 778.8373 & 3.3 \\
\hline b18 ${ }^{2+}$ & 827.3662 & 827.3639 & 2.8 \\
\hline b192+ & 884.8797 & 884.8777 & 2.2 \\
\hline b20 ${ }^{2+}$ & 958.4139 & 958.4118 & 2.2 \\
\hline y2 & 279.1703 & 279.1692 & 3.9 \\
\hline y3 & 394.1973 & 394.1962 & 2.7 \\
\hline y4 & 491.2500 & 491.2492 & 1.6 \\
\hline$y 17^{2+}$ & 823.3657 & 823.3633 & 3.0 \\
\hline$y 18^{2+}$ & 873.8895 & 873.8880 & 1.8 \\
\hline$y 19^{2+}$ & 931.4030 & 931.4027 & 0.3 \\
\hline$y 20^{2+}$ & 959.9137 & 959.9147 & 1.0 \\
\hline
\end{tabular}


B) HRMS/MS of fully methanolized doubly modified product formed by ThtA-D14A variant. A. Fragmentation of doubly modified product of ThtA-D14A variant after complete methanolysis. Green denotes the substituted position. Purple indicates an Asp methyl ester. Observed b- and $y$-ions are annotated. B. Annotated CID spectrum of fully methanolized doubly modified product of ThtA-D14A variant. C. Table of daughter ion assignments.

A

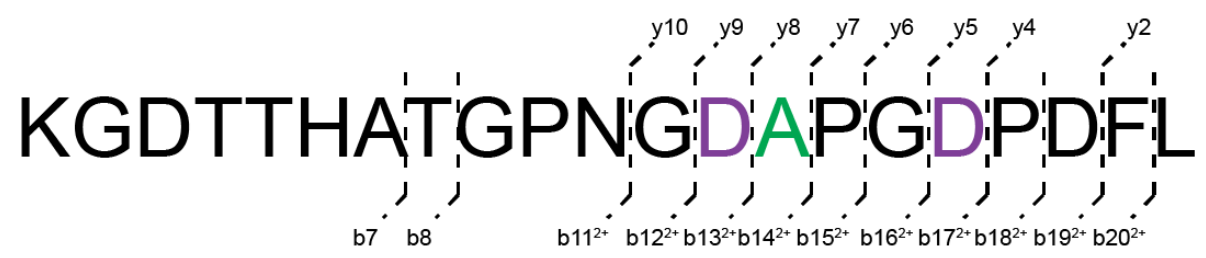

B

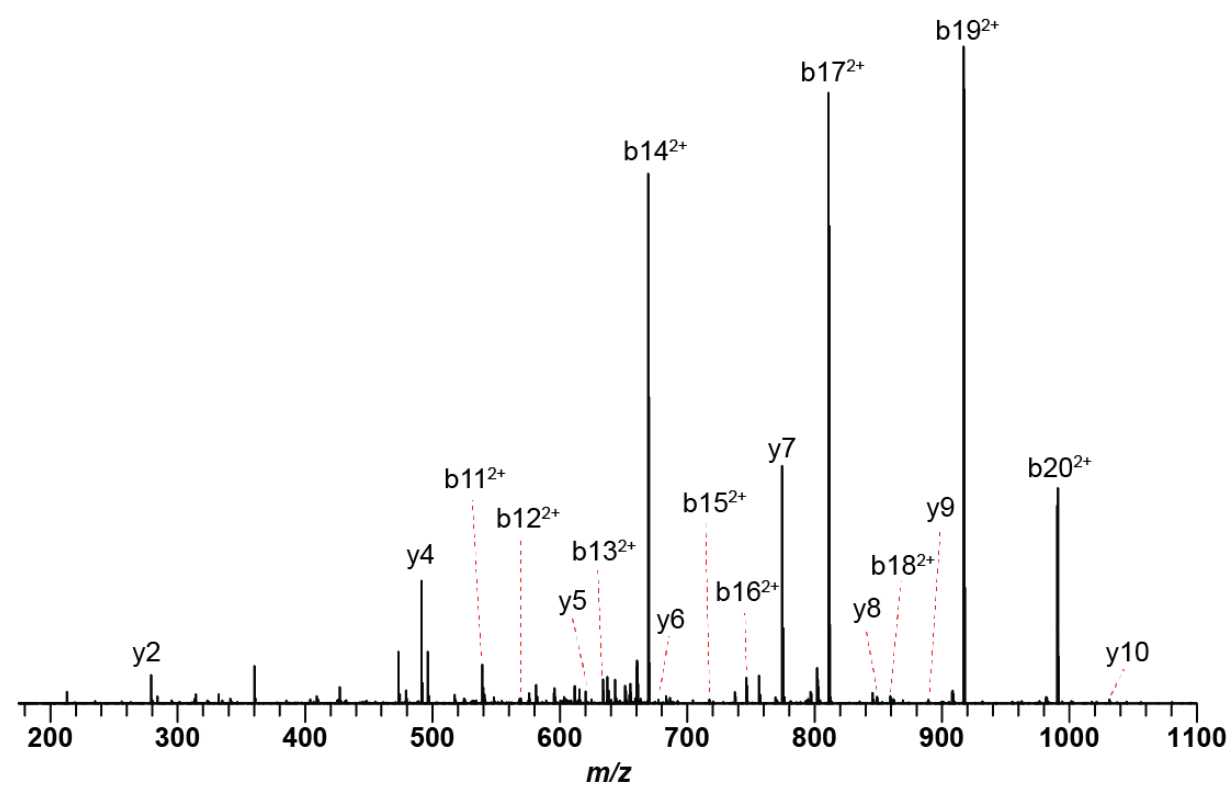

C

\begin{tabular}{|c|c|c|c|}
\hline Ion & Calc. Mass & Obs. Mass & Error (ppm) \\
\hline$[\mathrm{M}+3 \mathrm{H}]^{3+}$ & 704.3274 & 704.3270 & 0.6 \\
\hline b7 & 711.3420 & 711.3399 & 3.0 \\
\hline b8 & 812.3897 & 812.3868 & 3.6 \\
\hline b11 $1^{2+}$ & 540.7571 & 540.7564 & 1.2 \\
\hline b12 $2^{2+}$ & 569.2678 & 569.2670 & 1.5 \\
\hline b1 $3^{2+}$ & 633.7891 & 633.7882 & 1.4 \\
\hline b14 $4^{2+}$ & 669.3077 & 669.3069 & 1.1 \\
\hline b1 $5^{2+}$ & 717.8340 & 717.8324 & 2.3 \\
\hline b1 $6^{2+}$ & 746.3448 & 746.3431 & 2.2 \\
\hline b1 $7^{2+}$ & 810.8661 & 810.8634 & 3.3 \\
\hline b1 $8^{2+}$ & 859.3924 & 859.3906 & 2.2 \\
\hline
\end{tabular}

\begin{tabular}{cccc}
\hline lon & Calc. Mass & Obs. Mass & Error $(\mathbf{p p m})$ \\
\hline b19 $^{2+}$ & 916.9059 & 916.9040 & 2.1 \\
b20 $^{2+}$ & 990.4401 & 990.4379 & 2.2 \\
y2 & 279.1703 & 279.1692 & 4.0 \\
y4 & 491.2500 & 491.2491 & 1.9 \\
y5 & 620.2926 & 620.2916 & 1.6 \\
y6 & 677.3141 & 677.3122 & 2.8 \\
y7 & 774.3669 & 774.3640 & 3.7 \\
y8 & 845.4040 & 845.4017 & 2.6 \\
y9 & 974.4466 & 974.4444 & 2.2 \\
y10 & 1031.4680 & 1031.4660 & 2.0 \\
\hline
\end{tabular}


C) HRMS/MS of partially methanolized doubly modified product produced by ThtA-D14A variant. A. Fragmentation of partially methanolized doubly modified product of ThtA-D14A variant. Observed $b$ - and $y$-ions are annotated. Green denotes the substituted position. Purple indicates an Asp methyl ester. The black bracket connecting Thr and Asp indicates the site of macrolactone formation. B. Annotated CID spectrum of partially methanolized doubly modified product of ThtA-D14A variant. C. Table of daughter ion assignments.

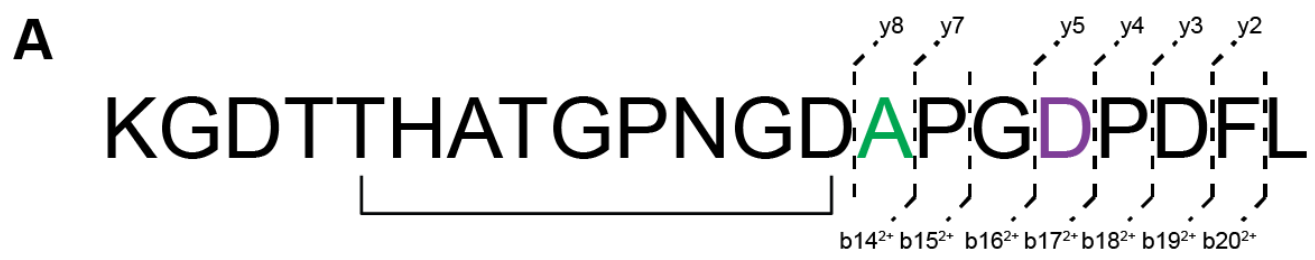

B

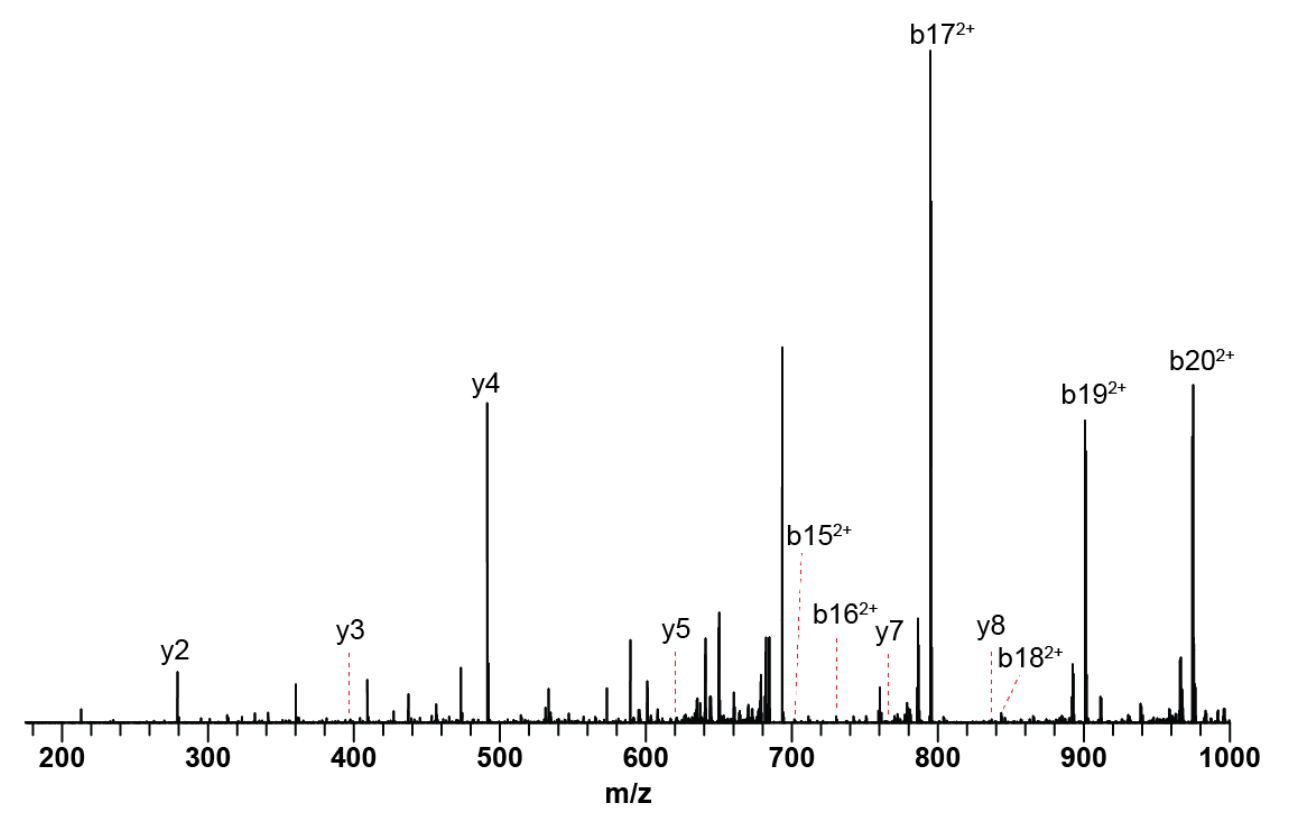

C

\begin{tabular}{|c|c|c|c|}
\hline Ion & Calc. Mass & Obs. Mass & Error (ppm) \\
\hline$[\mathrm{M}+3 \mathrm{H}]^{3+}$ & 693.6520 & 693.6516 & 0.5 \\
\hline b14 ${ }^{2+}$ & 653.2945 & 653.2938 & 1.2 \\
\hline b15 $5^{2+}$ & 701.8209 & 701.8191 & 2.6 \\
\hline b16 ${ }^{2+}$ & 730.3317 & 730.3303 & 1.8 \\
\hline b1 $17^{2+}$ & 794.8530 & 794.8504 & 3.2 \\
\hline b18 ${ }^{2+}$ & 843.3793 & 843.3777 & 2.0 \\
\hline b19 ${ }^{2+}$ & 900.8928 & 900.8910 & 2.1 \\
\hline b20 ${ }^{2+}$ & 974.4270 & 974.4251 & 2.0 \\
\hline y2 & 279.1703 & 279.1692 & 4.0 \\
\hline y3 & 394.1973 & 394.1962 & 2.8 \\
\hline
\end{tabular}

\begin{tabular}{cccc}
\hline Ion & Calc. Mass & Obs. Mass & Error (ppm) \\
\hline y4 & 491.2500 & 491.2492 & 1.7 \\
y5 & 620.2926 & 620.2915 & 1.9 \\
y7 & 774.3669 & 774.3640 & 3.6 \\
y8 & 845.4040 & 845.4015 & 2.9 \\
\hline
\end{tabular}


D) HRMS/MS of singly modified product formed by ThtA-D14A variant. A. Fragmentation of singly modified product formed upon co-expression of ThtA-D14A variant with ThtB. Observed band $y$-ions are annotated. Green denotes the substituted position. The black bracket connecting $\mathrm{Thr}$ and Asp indicates the site of macrolactone formation. B. Annotated CID spectrum of singly modified product. C. Table of daughter ion assignments.

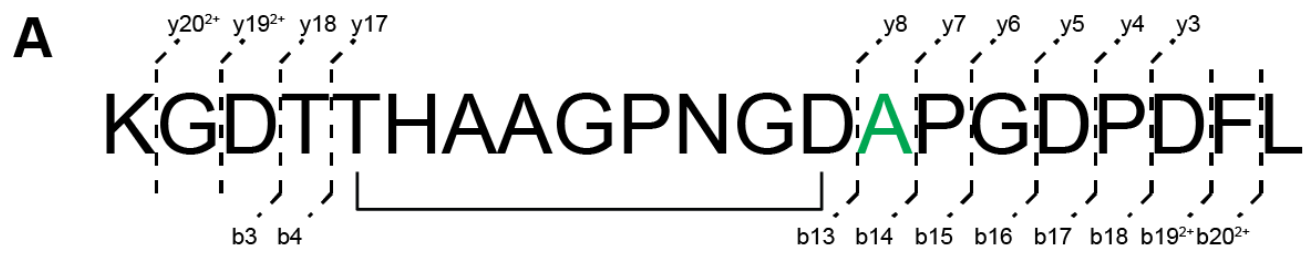

B

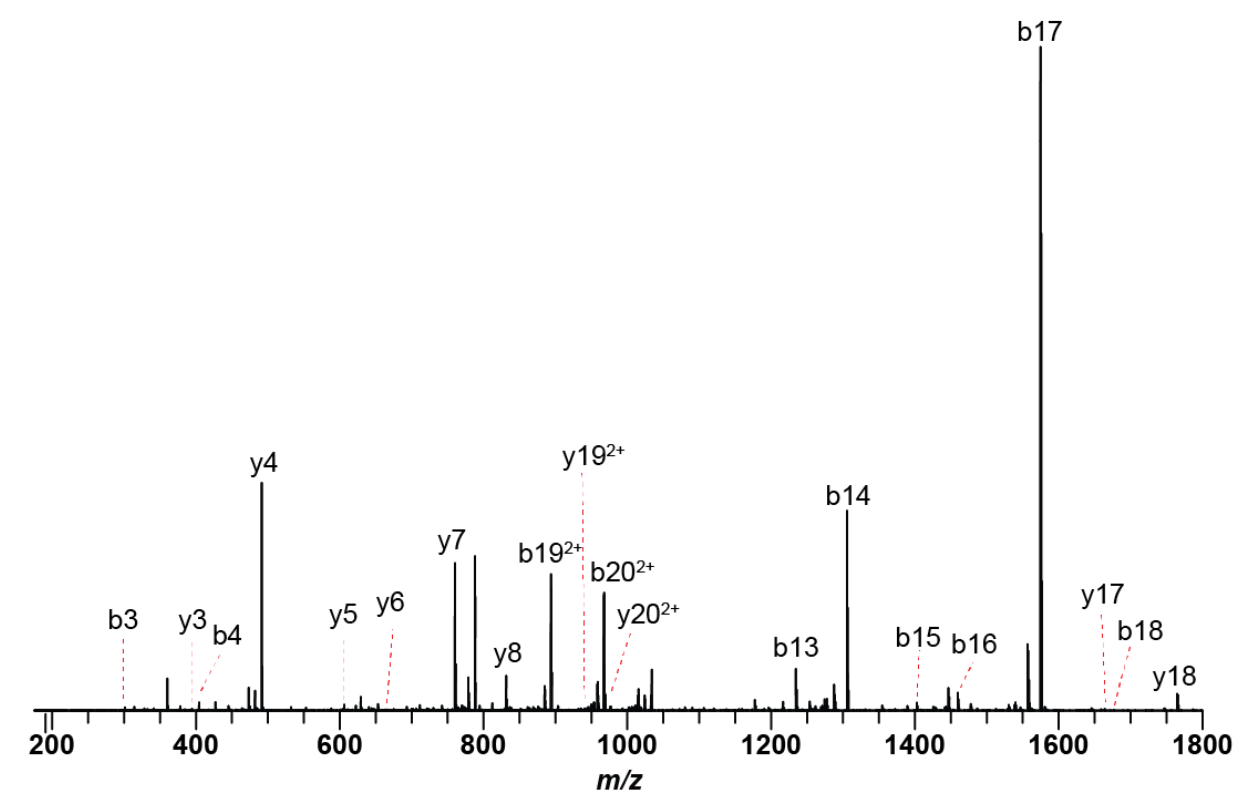

C

\begin{tabular}{|c|c|c|c|}
\hline Ion & Calc. Mass & Obs. Mass & Error (ppm) \\
\hline$[\mathrm{M}+3 \mathrm{H}]^{3+}$ & 1032.9665 & 1032.9660 & 0.5 \\
\hline b3 & 301.1507 & 301.1495 & 3.8 \\
\hline b4 & 402.1983 & 402.1973 & 2.5 \\
\hline b13 & 1234.5447 & 1234.5422 & 2.0 \\
\hline b14 & 1305.5818 & 1305.5797 & 1.6 \\
\hline b15 & 1402.6346 & 1402.6339 & 0.5 \\
\hline b16 & 1459.6560 & 1459.6535 & 1.7 \\
\hline b17 & 1574.6830 & 1574.6807 & 1.5 \\
\hline b18 & 1671.7358 & 1671.7370 & 0.7 \\
\hline b192+ & 893.8850 & 893.8833 & 1.9 \\
\hline b20 ${ }^{2+}$ & 967.4192 & 967.4173 & 1.9 \\
\hline
\end{tabular}

\begin{tabular}{cccc}
\hline Ion & Calc. Mass & Obs. Mass & Error $(\mathbf{p p m})$ \\
\hline y3 & 394.1973 & 394.1961 & 2.9 \\
y4 & 491.2500 & 491.2491 & 1.9 \\
y5 & 606.2770 & 606.2762 & 1.2 \\
y6 & 663.2984 & 663.2977 & 1.1 \\
y7 & 760.3512 & 760.3485 & 3.5 \\
y8 & 831.3883 & 831.3859 & 2.9 \\
y17 & 1663.7347 & 1663.7305 & 2.5 \\
y18 & 1764.7824 & 1764.7786 & 2.1 \\
y192+ & 940.4083 & 940.4054 & 3.1 \\
y20 $^{2+}$ & 968.9190 & 968.9172 & 1.9 \\
\hline
\end{tabular}


E) HRMS/MS of methanolized singly modified product formed by ThtA-D14A variant. A. Fragmentation of methanolized singly modified product formed upon co-expression of ThtA-D14A variant with ThtB. Observed b- and y-ions are annotated. Green denotes the substituted position. Purple denotes an Asp methyl ester. B. Annotated CID spectrum of the fully methanolized singly modified product. C. Table of daughter ion assignments

A

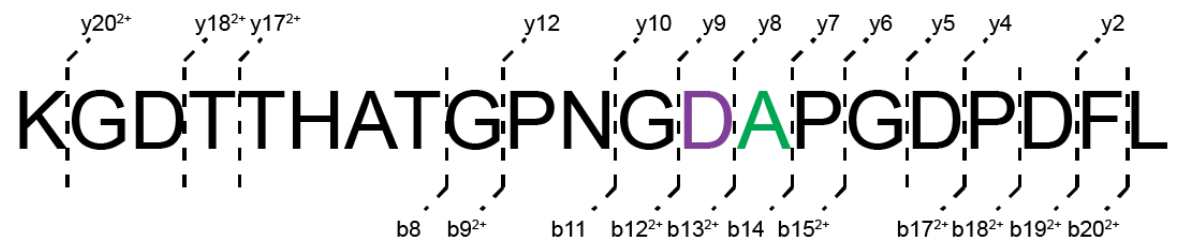

B

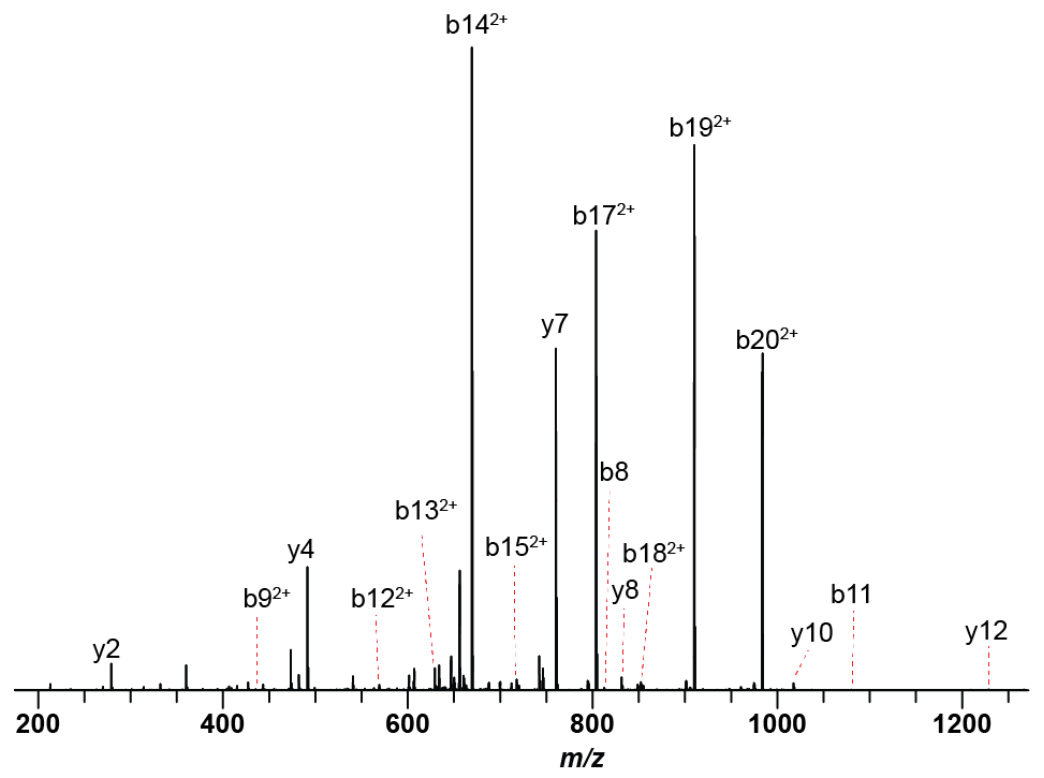

C

\begin{tabular}{|c|c|c|c|}
\hline Ion & Calc. Mass & Obs. Mass & Error (ppm) \\
\hline$[\mathrm{M}+3 \mathrm{H}]^{3+}$ & 699.6556 & 699.6553 & 0.3 \\
\hline b8 & 812.3897 & 812.3871 & 3.3 \\
\hline b9 $9^{2+}$ & 435.2092 & 435.2081 & 2.5 \\
\hline b11 & 1080.5069 & 1080.5053 & 1.4 \\
\hline b12 ${ }^{2+}$ & 569.2678 & 569.2671 & 1.3 \\
\hline b13 ${ }^{2+}$ & 633.7891 & 633.7883 & 1.2 \\
\hline b14 ${ }^{2+}$ & 669.3077 & 669.3070 & 1.0 \\
\hline b15 ${ }^{2+}$ & 717.8340 & 717.8324 & 2.2 \\
\hline b1 $7^{2+}$ & 803.8582 & 803.8557 & 3.1 \\
\hline b18 ${ }^{2+}$ & 852.3846 & 852.3827 & 2.2 \\
\hline b192+ & 909.8981 & 909.8963 & 1.9 \\
\hline b20 ${ }^{2+}$ & 983.4323 & 983.4302 & 2.2 \\
\hline
\end{tabular}

\begin{tabular}{cccc}
\hline Ion & Calc. Mass & Obs. Mass & Error (ppm) \\
\hline y4 & 491.2500 & 491.2492 & 1.6 \\
y5 & 606.2770 & 606.2764 & 1.0 \\
y6 & 663.2984 & 663.2978 & 1.0 \\
y7 & 760.3512 & 760.3485 & 3.6 \\
y8 & 831.3883 & 831.3859 & 2.9 \\
\hline y10 & 1017.4524 & 1017.4504 & 1.9 \\
y12 & 1228.5481 & 1228.5451 & 2.4 \\
y17 $^{2+}$ & 848.3841 & 848.3810 & 3.6 \\
y18 $^{2+}$ & 898.9079 & 898.9063 & 1.8 \\
y20 $^{2+}$ & 984.9321 & 984.9287 & 3.5 \\
\hline
\end{tabular}


Figure S25. ThtA-D19A variant-modified products: HRMS/MS.

A) HRMS/MS of doubly modified product-1 formed by ThtA-D19A variant. A. Fragmentation of doubly modified product-1 of ThtA-D19A variant. Observed b- and y-ions are annotated. Green denotes the substituted position. Black brackets connecting Thr and Asp indicate sites of macrolactone formation. B. Annotated CID spectrum of doubly modified product-1 of ThtA-D19A variant. C. Table of daughter ion assignments.

A

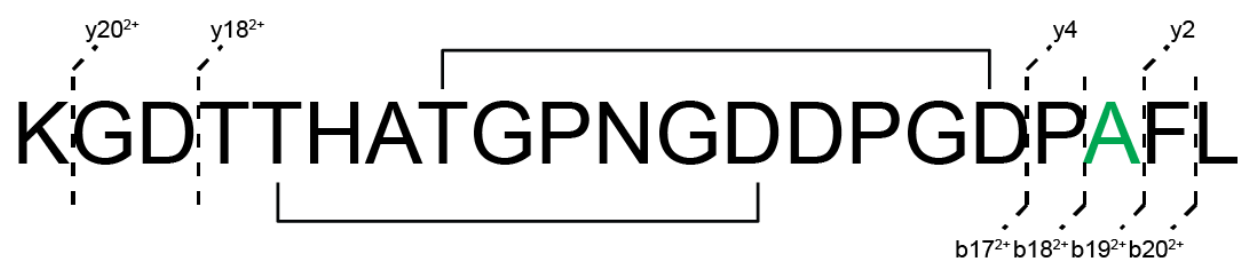

B

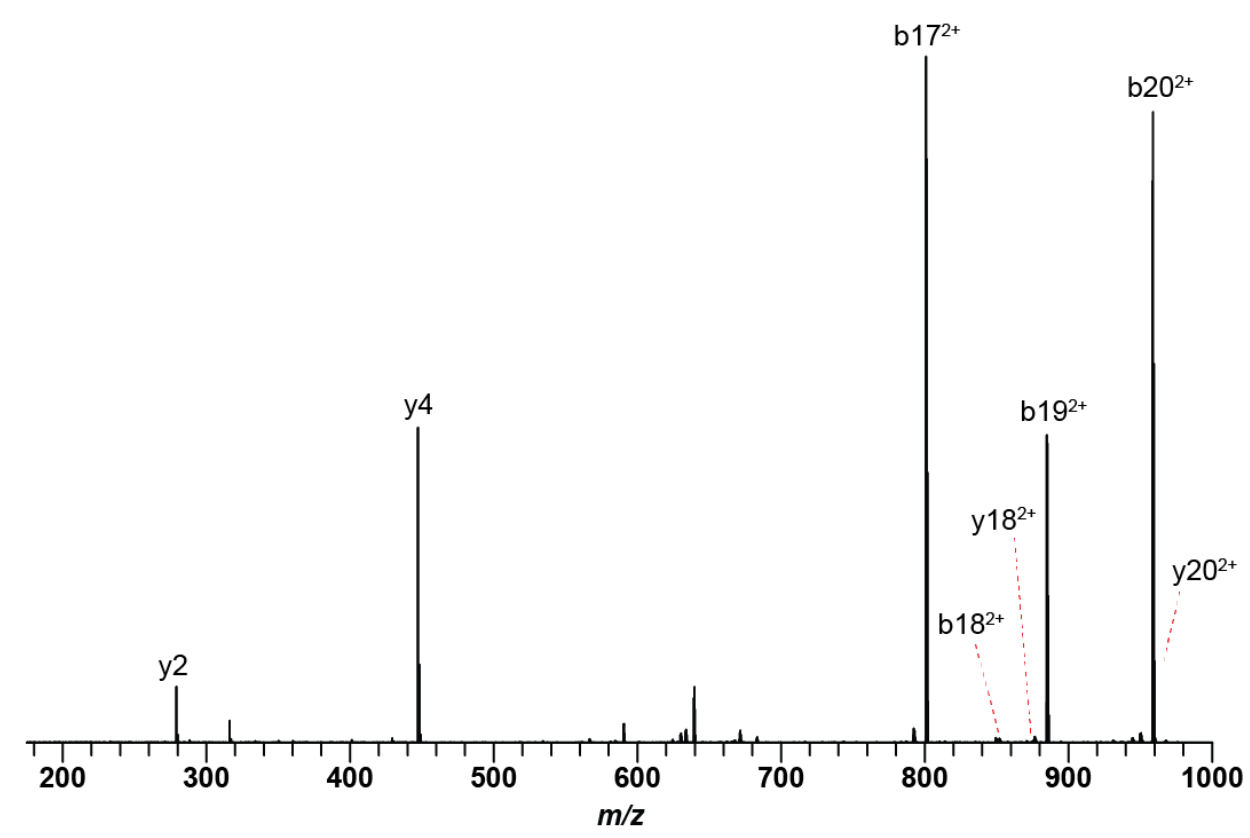

\begin{tabular}{|c|c|c|c|}
\hline Ion & Calc. Mass & Obs. Mass & Error (ppm) \\
\hline$[\mathrm{M}+3 \mathrm{H}]^{3+}$ & 682.97658 & 682.97640 & 0.3 \\
\hline b1 $17^{2+}$ & 800.83476 & 800.83240 & 2.9 \\
\hline b18 $8^{2+}$ & 849.36115 & 849.35930 & 2.2 \\
\hline b1 $19^{2+}$ & 884.87970 & 884.87790 & 2.0 \\
\hline b20 $0^{2+}$ & 958.41391 & 958.41200 & 2.0 \\
\hline y2 & 279.17032 & 279.16920 & 4.0 \\
\hline y4 & 447.26020 & 447.25900 & 2.7 \\
\hline$y 18^{2+}$ & 873.88954 & 873.88710 & 2.8 \\
\hline y20 ${ }^{2+}$ & 959.91374 & 959.9151 & 1.4 \\
\hline
\end{tabular}


B) HRMS/MS of fully methanolized doubly modified product-1 formed by ThtA-D19A variant. A. Fragmentation of doubly modified product-1 of ThtA-D19A variant after complete methanolysis. Green denotes the substituted position. Purple indicates an Asp methyl ester. Observed $b$ - and $y$-ions are annotated. B. Annotated CID spectrum of fully methanolized doubly modified product-1 of ThtA-D19A variant. C. Table of daughter ion assignments.

A

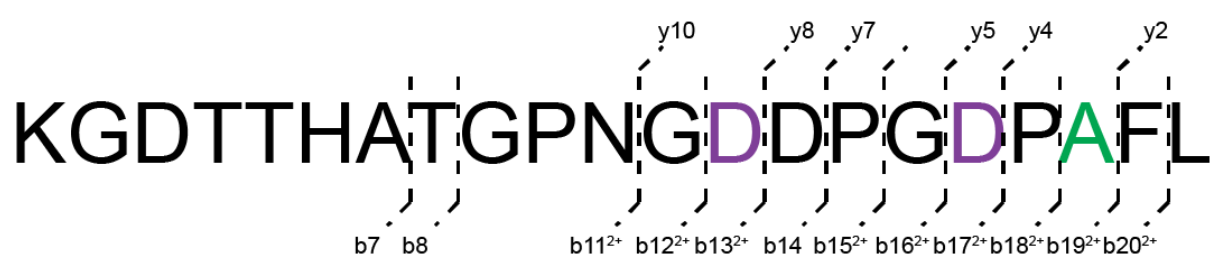

B

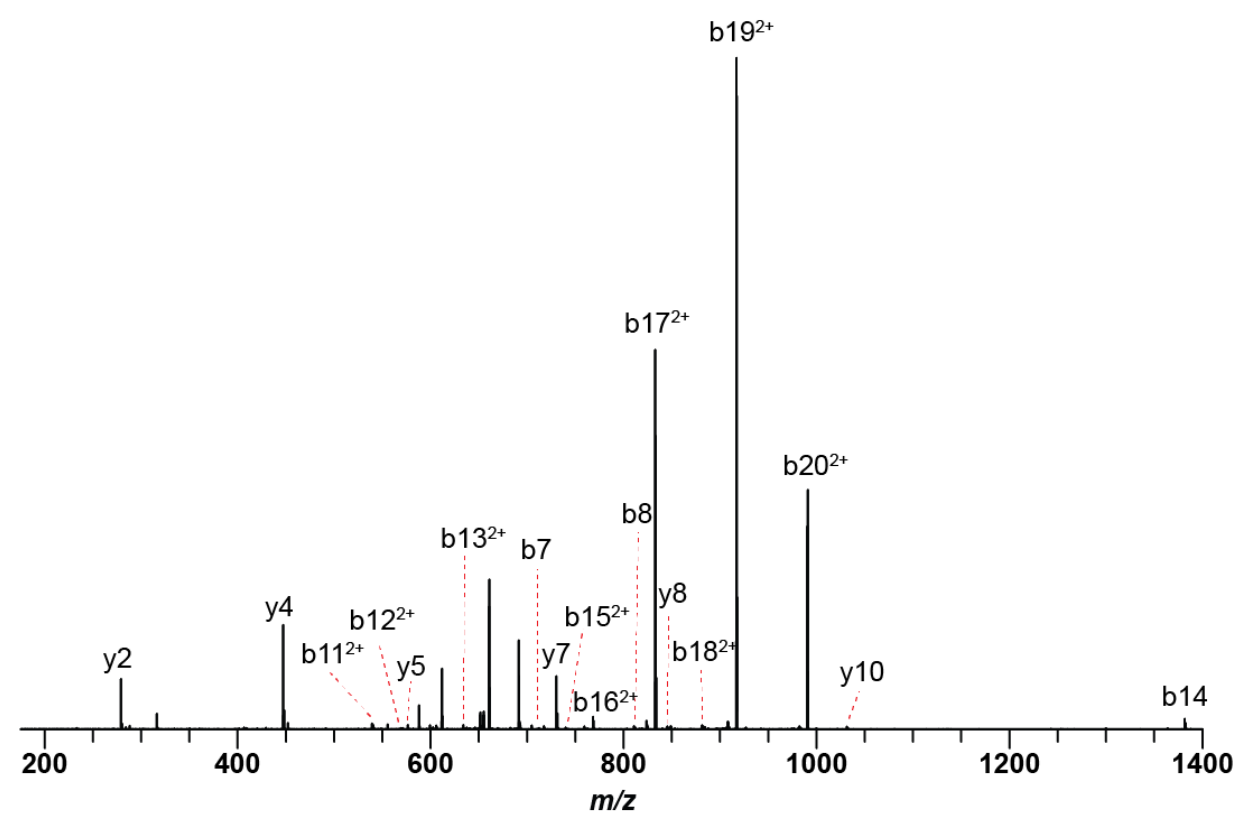

C

\begin{tabular}{|c|c|c|c|}
\hline Ion & Calc. Mass & Obs. Mass & Error (ppm) \\
\hline$[\mathrm{M}+3 \mathrm{H}]^{3+}$ & 704.3274 & 704.3270 & 0.6 \\
\hline b7 & 711.3420 & 711.3404 & 2.3 \\
\hline b8 & 812.3897 & 812.3875 & 2.7 \\
\hline b11 ${ }^{2+}$ & 540.7571 & 540.7564 & 1.2 \\
\hline b12 $2^{2+}$ & 569.2678 & 569.2670 & 1.4 \\
\hline b13 ${ }^{2+}$ & 633.7891 & 633.7883 & 1.3 \\
\hline b14 & 1381.5979 & 1381.5962 & 1.2 \\
\hline b1 $15^{2+}$ & 739.8290 & 739.8272 & 2.4 \\
\hline b1 $6^{2+}$ & 768.3397 & 768.3371 & 3.4 \\
\hline b1 $17^{2+}$ & 832.8610 & 832.8584 & 3.1 \\
\hline
\end{tabular}

\begin{tabular}{cccc}
\hline Ion & Calc. Mass & Obs. Mass & Error $(\mathbf{p p m})$ \\
\hline b18 $^{2+}$ & 881.3874 & 881.3856 & 2.0 \\
b19 $^{2+}$ & 916.9059 & 916.9044 & 1.7 \\
b20 $^{2+}$ & 990.4401 & 990.4383 & 1.8 \\
\hline y2 & 279.1703 & 279.1692 & 4.0 \\
y4 & 447.2602 & 447.2592 & 2.2 \\
y5 & 576.3028 & 576.3020 & 1.4 \\
y7 & 730.3770 & 730.3754 & 2.2 \\
y8 & 845.4040 & 845.4018 & 2.6 \\
y10 & 1031.4680 & 1031.4662 & 1.8 \\
\hline
\end{tabular}


C) HRMS/MS of partially methanolized doubly modified product-1 formed by ThtA-D19A variant. A. Fragmentation of partially methanolized doubly modified product-1 of ThtA-D19A variant. Observed $b$ - and $y$-ions are annotated. Green denotes the substituted position. Purple indicates an Asp methyl ester. The black bracket connecting Thr and Asp indicates the site of macrolactone formation. B. Annotated CID spectrum of partially methanolized thatisin A D19A variant. C. Table of daughter ion assignments.

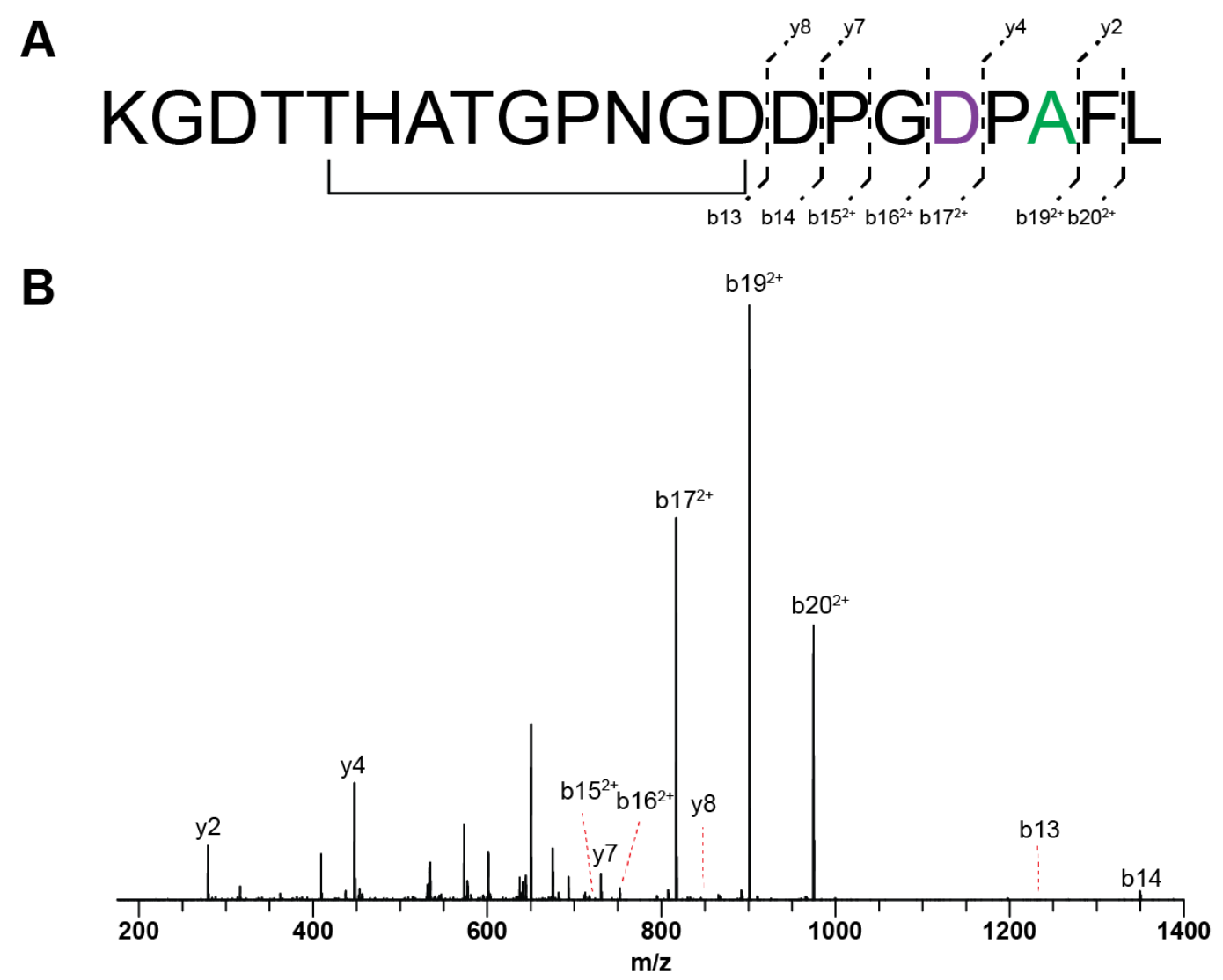

\begin{tabular}{|c|c|c|c|}
\hline Ion & Calc. Mass & Obs. Mass & Error (ppm) \\
\hline$[\mathrm{M}+3 \mathrm{H}]^{3+}$ & 693.6520 & 693.6517 & 0.4 \\
\hline b13 & 1234.5447 & 1234.5424 & 1.9 \\
\hline b14 & 1349.5717 & 1349.5696 & 1.5 \\
\hline b15 ${ }^{2+}$ & 723.8158 & 723.8143 & 2.1 \\
\hline b16 ${ }^{2+}$ & 752.3266 & 752.3240 & 3.4 \\
\hline b1 $17^{2+}$ & 816.8479 & 816.8454 & 3.0 \\
\hline b192+ & 900.8928 & 900.8911 & 1.9 \\
\hline b20 ${ }^{2+}$ & 974.4270 & 974.4251 & 2.0 \\
\hline y2 & 279.1703 & 279.1693 & 3.7 \\
\hline y4 & 447.2602 & 447.2592 & 2.2 \\
\hline
\end{tabular}

\begin{tabular}{cccc}
\hline Ion & Calc. Mass & Obs. Mass & Error (ppm) \\
\hline y7 & 730.3770 & 730.3753 & 2.4 \\
y8 & 845.4040 & 845.4020 & 2.3 \\
\hline
\end{tabular}


D) HRMS/MS of doubly modified product-2 formed by ThtA-D19A variant. A. Fragmentation of doubly modified product-2 of ThtA-D19A variant. Observed b- and y-ions are annotated. Green denotes the substituted position. Black brackets connecting Thr and Asp indicate sites of macrolactone formation. B. Annotated CID spectrum of doubly modified product-2 of ThtA-D19A variant. C. Table of daughter ion assignments.

A

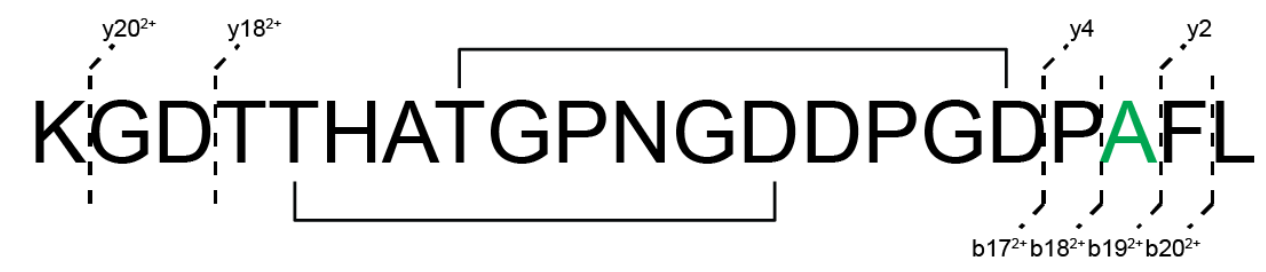

B

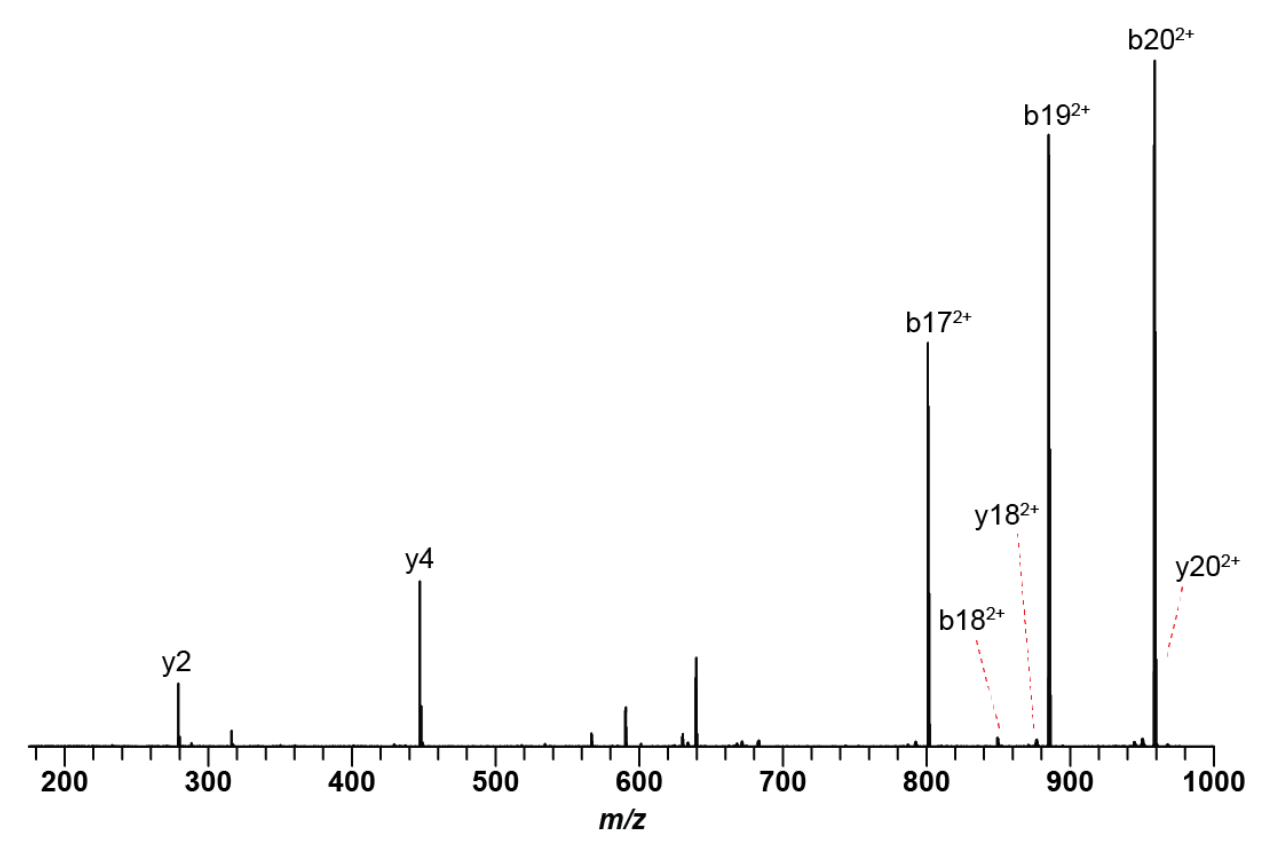

\begin{tabular}{|c|c|c|c|}
\hline Ion & Calc. Mass & Obs. Mass & Error (ppm) \\
\hline$[\mathrm{M}+3 \mathrm{H}]^{3+}$ & 682.97658 & 682.97650 & 0.1 \\
\hline b1 $17^{2+}$ & 800.83476 & 800.83230 & 3.1 \\
\hline b18 ${ }^{2+}$ & 849.36115 & 849.35940 & 2.1 \\
\hline b19 ${ }^{2+}$ & 884.87970 & 884.87810 & 1.8 \\
\hline b20 ${ }^{2+}$ & 958.41391 & 958.41210 & 1.9 \\
\hline y2 & 279.17032 & 279.16930 & 3.7 \\
\hline y4 & 447.26020 & 447.25910 & 2.5 \\
\hline$y 18^{2+}$ & 873.88954 & 873.88790 & 1.9 \\
\hline$y 20^{2+}$ & 959.91374 & 959.9151 & 1.4 \\
\hline
\end{tabular}


E) HRMS/MS of fully methanolized doubly modified product-2 formed by ThtA-D19A variant. A. Fragmentation of doubly modified product-2 of ThtA-D19A variant after complete methanolysis. Green denotes the substituted position. Purple indicates an Asp methyl ester. Observed $b$ - and $y$-ions are annotated. B. Annotated CID spectrum of fully methanolized doubly modified product-2 of ThtA-D19A variant. C. Table of daughter ion assignments.

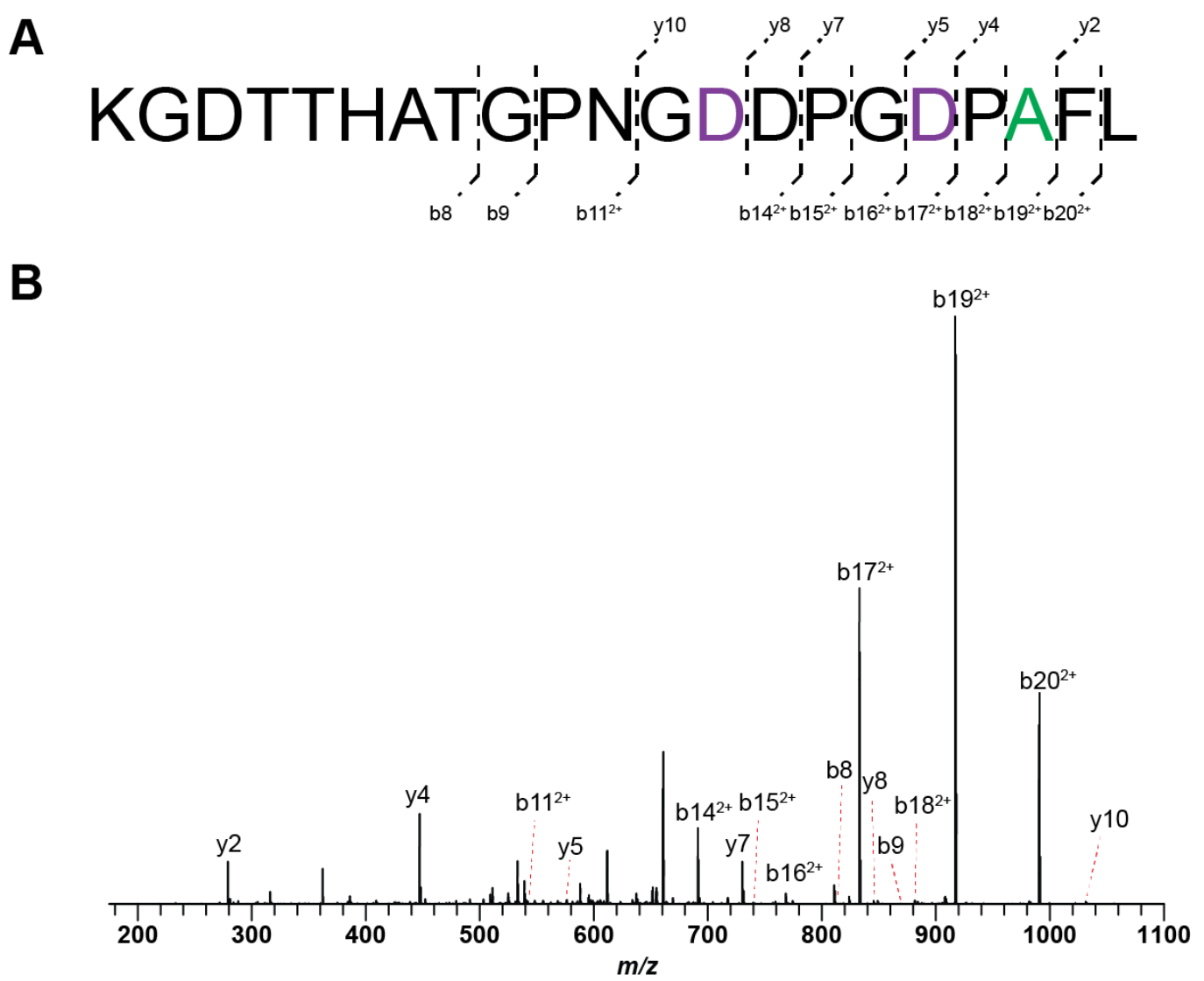

\begin{tabular}{cccc}
$\mathbf{C}$ & \multicolumn{3}{l}{} \\
\cline { 2 - 4 } lon & Calc. Mass & Obs. Mass & Error $(\mathbf{p p m})$ \\
\hline$[\mathrm{M+3H}]^{3+}$ & 704.3274 & 704.3270 & 0.6 \\
b8 & 812.3897 & 812.3875 & 2.7 \\
b9 & 869.4112 & 869.4088 & 2.7 \\
b11 $^{2+}$ & 540.7571 & 540.7563 & 1.4 \\
b14 $^{2+}$ & 691.3026 & 691.3008 & 2.6 \\
b15 $^{2+}$ & 739.8290 & 739.8270 & 2.6 \\
b16 $^{2+}$ & 768.3397 & 768.3369 & 3.6 \\
\hline b17 $^{2+}$ & 832.8610 & 832.8584 & 3.1 \\
\hline b18 $^{2+}$ & 881.3874 & 881.3855 & 2.1 \\
\hline b19 $^{2+}$ & 916.9059 & 916.9040 & 2.1 \\
\hline
\end{tabular}

\begin{tabular}{cccc}
\hline Ion & Calc. Mass & Obs. Mass & Error $(\mathbf{p p m})$ \\
\hline b20 $^{2+}$ & 990.4401 & 990.4380 & 2.1 \\
y2 $^{2}$ & 279.1703 & 279.1692 & 4.0 \\
y4 & 447.2602 & 447.2591 & 2.5 \\
y5 & 576.3028 & 576.3021 & 1.2 \\
y7 & 730.3770 & 730.3754 & 2.2 \\
y8 & 845.4040 & 845.4016 & 2.8 \\
y10 & 1031.4680 & 1031.4657 & 2.2 \\
\hline
\end{tabular}


F) HRMS/MS of partially methanolized doubly modified product-2 formed by ThtA-D19A variant. A. Fragmentation of partially methanolized doubly modified product-2 of ThtA-D19A variant. Observed $b$ - and $y$-ions are annotated. Green denotes the substituted position. Purple indicates an Asp methyl ester. The black bracket connecting Thr and Asp indicates the site of macrolactone formation. B. Annotated CID spectrum of partially methanolized doubly modified product-2 of ThtA-D19A variant. C. Table of daughter ion assignments.

A

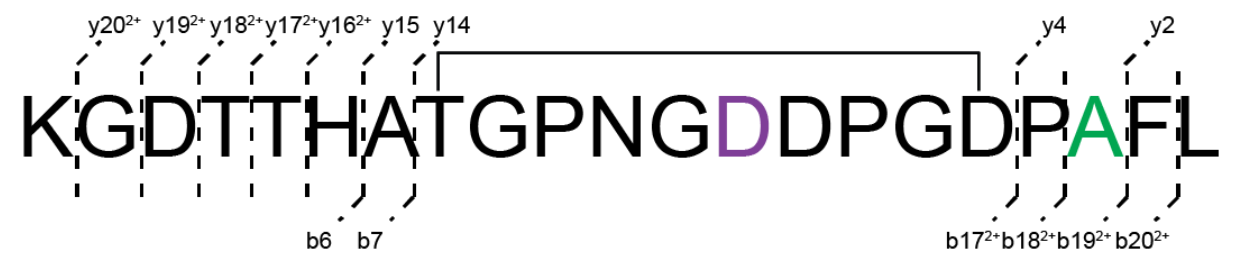

B

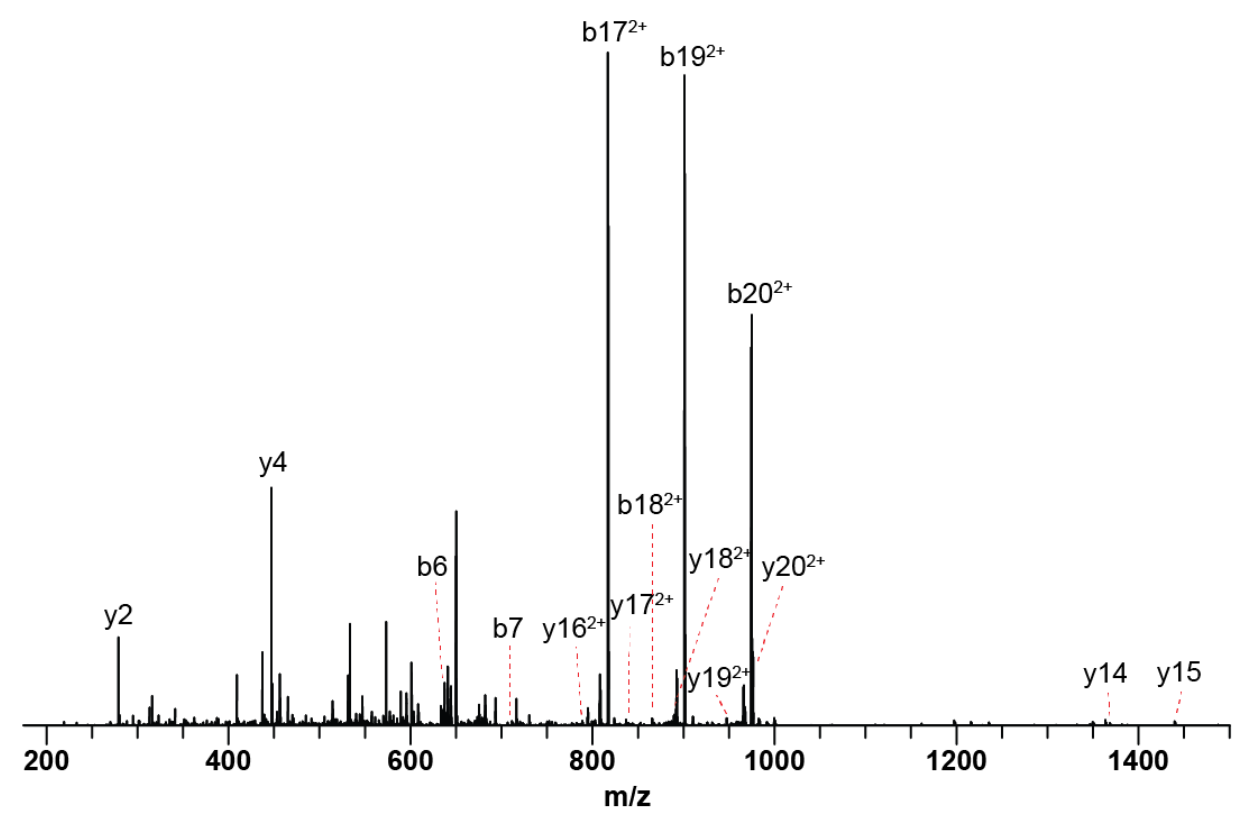

C

\begin{tabular}{|c|c|c|c|}
\hline Ion & Calc. Mass & Obs. Mass & Error (ppm) \\
\hline$[\mathrm{M}+3 \mathrm{H}]^{3+}$ & 693.6520 & 693.6516 & 0.5 \\
\hline b6 & 640.3049 & 640.3041 & 1.3 \\
\hline b7 & 711.3420 & 711.3407 & 1.9 \\
\hline b1 $17^{2+}$ & 816.8479 & 816.8454 & 3.0 \\
\hline b18 ${ }^{2+}$ & 865.3743 & 865.3723 & 2.3 \\
\hline b19 ${ }^{2+}$ & 900.8928 & 900.8909 & 2.1 \\
\hline b20 ${ }^{2+}$ & 974.4270 & 974.4251 & 2.0 \\
\hline y2 & 279.1703 & 279.1692 & 4.0 \\
\hline y4 & 447.2602 & 447.2592 & 2.2 \\
\hline y14 & 1368.6066 & 1368.6054 & 0.9 \\
\hline
\end{tabular}

\begin{tabular}{cccc}
\hline Ion & Calc. Mass & Obs. Mass & Error $(\mathbf{p p m})$ \\
\hline $\mathbf{y 1 5}$ & 1439.6438 & 1439.6421 & 1.1 \\
$\mathbf{y} 16^{2+}$ & 788.8550 & 788.8526 & 3.0 \\
$\mathbf{y} 17^{2+}$ & 839.3788 & 839.3764 & 2.9 \\
$\mathbf{y 1 8}^{2+}$ & 889.9026 & 889.9009 & 2.0 \\
$\mathbf{y 1 9}^{2+}$ & 947.4161 & 947.4145 & 1.7 \\
$\mathbf{y 2 0 ^ { 2 + }}$ & 975.9268 & 975.9247 & 2.2 \\
\hline
\end{tabular}


G) HRMS/MS of singly modified product-1 formed by ThtA-D19A variant. A. Fragmentation of singly modified product-1 formed upon co-expression of ThtA-D19A variant with ThtB. Observed b- and y-ions are annotated. Green denotes the substituted position. The black bracket connecting Thr and Asp indicates the site of macrolactone formation. B. Annotated CID spectrum of singly modified product-1. C. Table of daughter ion assignments.

A

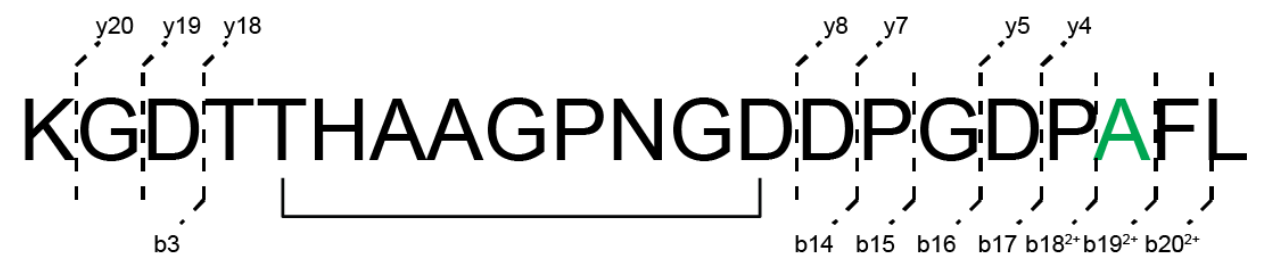

B

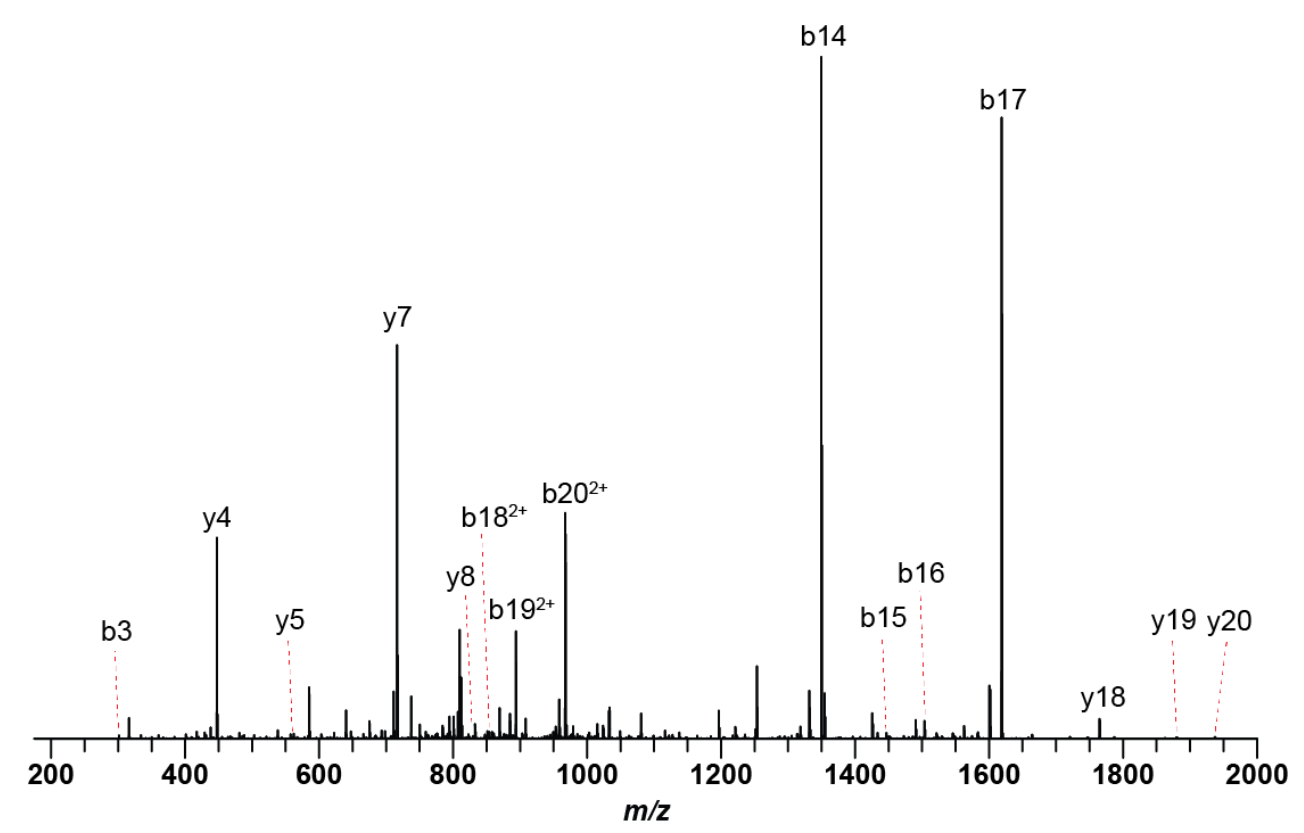

C

\begin{tabular}{cccc}
\hline Ion & Calc. Mass & Obs. Mass & Error $(\mathbf{p p m})$ \\
\hline${\text { [M+2H }]^{2+}}^{\mathbf{1}}$ & 1032.9665 & 1032.9658 & 0.7 \\
b3 & 301.1507 & 301.1496 & 3.5 \\
b14 & 1349.5717 & 1349.5705 & 0.9 \\
b15 & 1446.6244 & 1446.6227 & 1.2 \\
b16 & 1503.6459 & 1503.6447 & 0.8 \\
b17 & 1618.6728 & 1618.6719 & 0.6 \\
b18 $^{2+}$ & 858.3664 & 858.3645 & 2.2 \\
\hline b19 $^{2+}$ & 893.8850 & 893.8835 & 1.7 \\
\hline b20 & \\
\hline y4 & 967.4192 & 967.4175 & 1.7 \\
\hline
\end{tabular}

\begin{tabular}{cccc}
\hline Ion & Calc. Mass & Obs. Mass & Error (ppm) \\
\hline y5 & 562.2871 & 562.2863 & 1.5 \\
y7 & 716.3614 & 716.3597 & 2.3 \\
y8 & 831.3883 & 831.3862 & 2.5 \\
y18 & 1764.7824 & 1764.7798 & 1.5 \\
y19 & 1879.8093 & 1879.8082 & 0.6 \\
y20 & 1936.8308 & 1936.8258 & 2.6 \\
\hline
\end{tabular}


H) HRMS/MS of methanolized singly modified product-1 formed by ThtA-D19A variant. A. Fragmentation of methanolized singly modified product-1 formed upon co-expression of ThtAD19A variant with ThtB. Observed b- and y-ions are annotated. Green denotes the substituted position. Purple denotes an Asp methyl ester. B. Annotated CID spectrum of fully methanolized singly modified product-1. C. Table of daughter ion assignments.

A

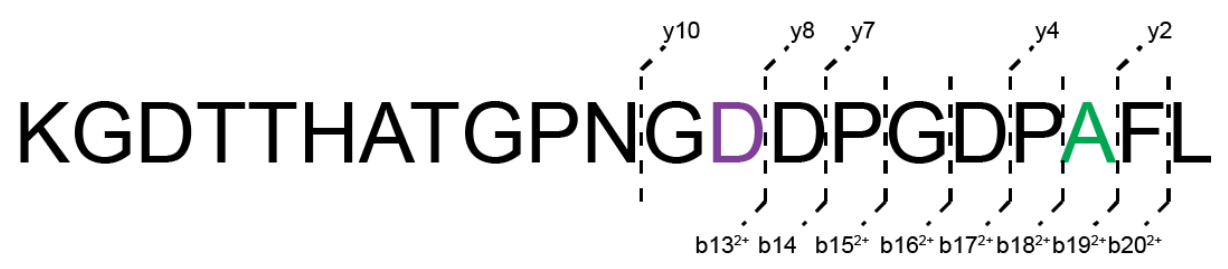

B

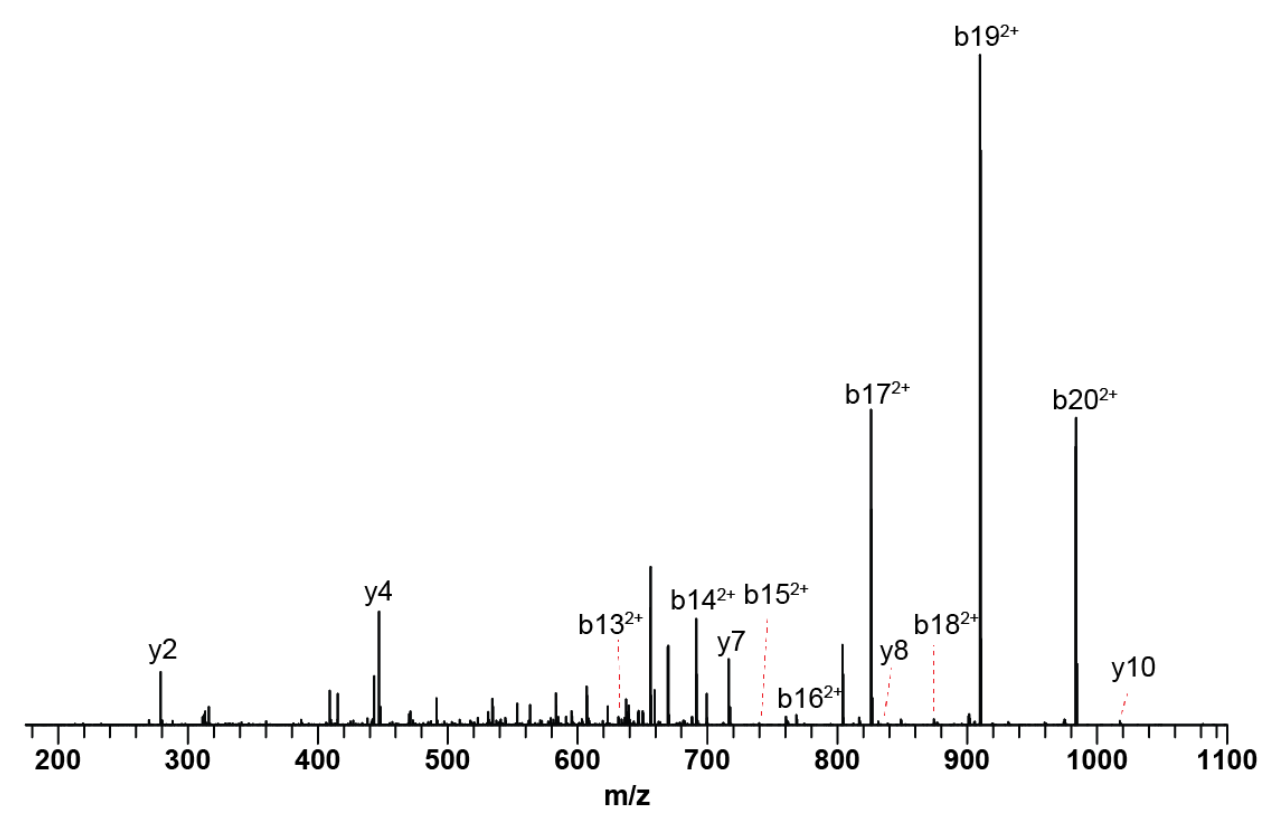

C

\begin{tabular}{|c|c|c|c|}
\hline Ion & Calc. Mass & Obs. Mass & Error (ppm) \\
\hline$[\mathrm{M}+3 \mathrm{H}]^{3+}$ & 699.6555 & 699.6551 & 0.6 \\
\hline b132+ & 633.7891 & 633.7883 & 1.3 \\
\hline b1 $4^{2+}$ & 691.3026 & 691.3006 & 2.8 \\
\hline b1 $5^{2+}$ & 739.8290 & 739.8276 & 1.8 \\
\hline b1 $6^{2+}$ & 768.3397 & 768.3366 & 4.0 \\
\hline b1 $7^{2+}$ & 825.8532 & 825.8505 & 3.2 \\
\hline b1 $8^{2+}$ & 874.3795 & 874.3777 & 2.1 \\
\hline b192+ & 909.8981 & 909.8964 & 1.9 \\
\hline b20 $2^{2+}$ & 983.4323 & 983.4304 & 1.9 \\
\hline y2 & 279.1703 & 279.1691 & 4.4 \\
\hline
\end{tabular}

\begin{tabular}{cccc}
\hline Ion & Calc. Mass & Obs. Mass & Error $(\mathbf{p p m})$ \\
\hline y4 & 447.2602 & 447.2590 & 2.7 \\
y7 & 716.3614 & 716.3596 & 2.5 \\
y8 & 831.3883 & 831.3854 & 3.5 \\
y10 & 1017.4524 & 1017.4508 & 1.5 \\
\hline
\end{tabular}


I) HRMS/MS of singly modified product-2 formed by ThtA-D19A variant. A. Fragmentation of singly modified product-2 formed upon co-expression of ThtA-D19A variant with ThtB. Observed b- and y-ions are annotated. Green denotes the substituted position. The black bracket connecting Thr and Asp indicates the site of macrolactone formation. B. Annotated CID spectrum of singly modified product-2. C. Table of daughter ion assignments.

A

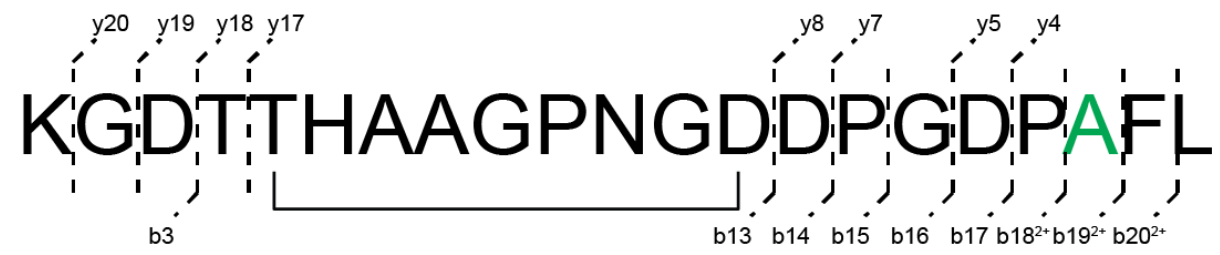

B

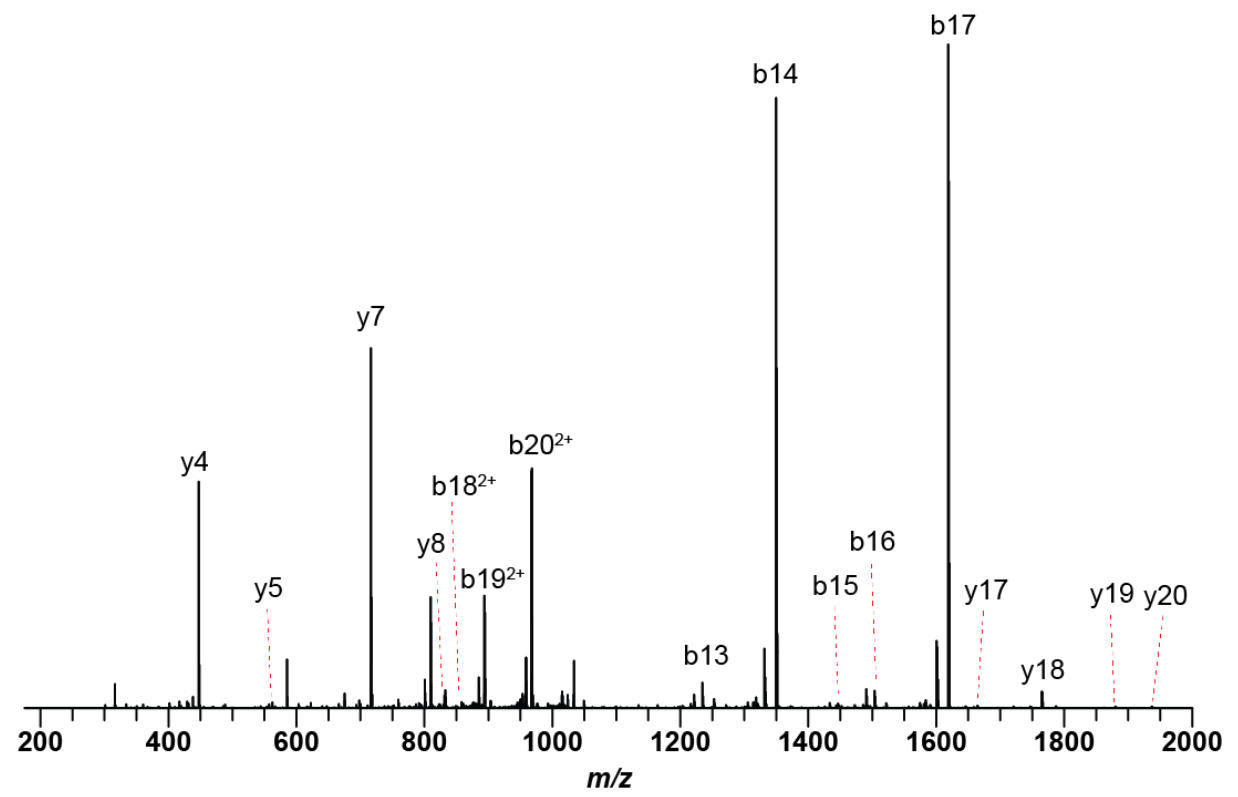

C

\begin{tabular}{cccc}
\hline lon & Calc. Mass & Obs. Mass & Error $(\mathbf{p p m})$ \\
\hline$[\mathbf{M + 2 H}]^{2+}$ & 1032.9665 & 1032.9656 & 0.9 \\
b3 & 301.1507 & 301.1494 & 4.2 \\
b13 & 1234.5447 & 1234.5420 & 2.2 \\
b14 & 1349.5717 & 1349.5698 & 1.4 \\
b15 & 1446.6244 & 1446.6220 & 1.7 \\
\hline b16 & 1503.6459 & 1503.6437 & 1.4 \\
\hline b17 & 1618.6728 & 1618.6704 & 1.5 \\
b18 $^{2+}$ & 858.3664 & 858.3641 & 2.7 \\
b19 $^{2+}$ & 893.8850 & 893.8830 & 2.2 \\
\hline b20 $^{2+}$ & 967.4192 & 967.4170 & 2.3 \\
\hline y4 & 447.2602 & 447.2591 & 2.5 \\
\hline
\end{tabular}

\begin{tabular}{cccc}
\hline Ion & Calc. Mass & Obs. Mass & Error $(\mathbf{p p m})$ \\
\hline y5 & 562.2871 & 562.2861 & 1.8 \\
y7 & 716.3614 & 716.3594 & 2.8 \\
y8 & 831.3883 & 831.3857 & 3.1 \\
y17 & 1663.7347 & 1663.7319 & 1.7 \\
y18 & 1764.7824 & 1764.7791 & 1.9 \\
y19 & 1879.8093 & 1879.8070 & 1.2 \\
y20 & 1936.8308 & 1936.8241 & 3.4 \\
\hline
\end{tabular}


J) HRMS/MS of methanolized singly modified product-2 formed by ThtA-D19A variant. A. Fragmentation of methanolized singly modified product-2 formed upon co-expression of ThtAD19A variant with ThtB. Observed b- and y-ions are annotated. Green denotes the substituted position. Purple denotes an Asp methyl ester. B. Annotated CID spectrum of fully methanolized singly modified product-2. C. Table of daughter ion assignments.

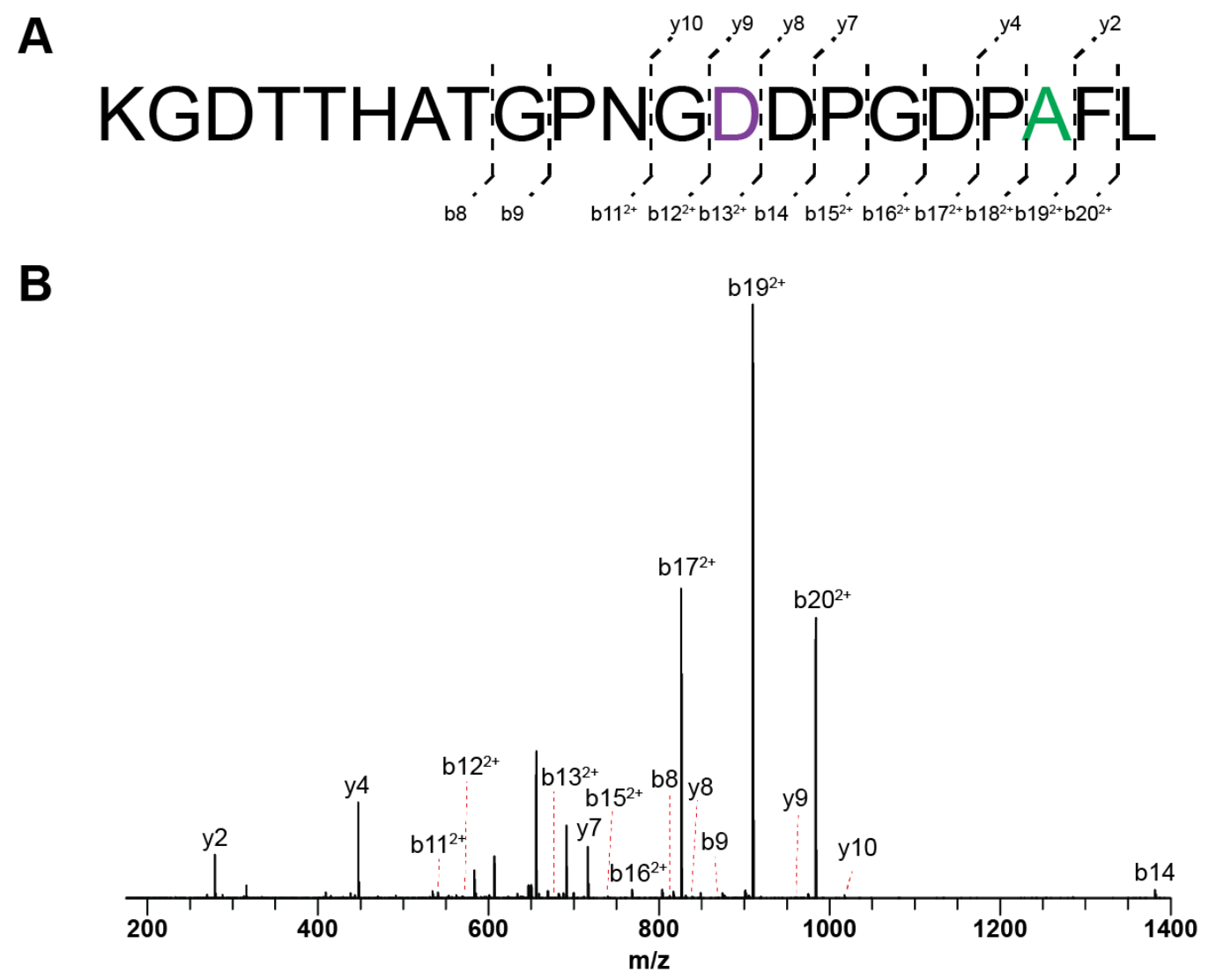

\begin{tabular}{cccc}
$\mathbf{C}$ & \multicolumn{3}{l}{} \\
\cline { 2 - 4 } lon & Calc. Mass & Obs. Mass & Error (ppm) \\
\hline$[\mathbf{M + 3 H}]^{3+}$ & 699.6555 & 699.6552 & 0.4 \\
\hline b8 & 812.3897 & 812.3871 & 3.2 \\
b9 & 869.4112 & 869.4090 & 2.5 \\
\hline b11 $^{2+}$ & 540.7571 & 540.7562 & 1.6 \\
b12 $^{2+}$ & 569.2678 & 569.2667 & 1.9 \\
\hline b13 $^{2+}$ & 633.7891 & 633.7881 & 1.6 \\
\hline b14 $^{2+}$ & 1381.5979 & 1381.5960 & 1.3 \\
b15 $^{2+}$ & 739.8290 & 739.8273 & 2.2 \\
\hline b16 $^{2+}$ & 768.3397 & 768.3367 & 3.9 \\
\hline b17 $^{2+}$ & 825.8532 & 825.8505 & 3.2 \\
\hline
\end{tabular}

\begin{tabular}{cccc}
\hline lon & Calc. Mass & Obs. Mass & Error $(\mathbf{p p m})$ \\
\hline b18 $^{2+}$ & 874.3795 & 874.3776 & 2.2 \\
b19 $^{2+}$ & 909.8981 & 909.8963 & 2.0 \\
b20 $^{2+}$ & 983.4323 & 983.4303 & 2.0 \\
\hline y2 & 279.1703 & 279.1692 & 4.0 \\
y4 & 447.2602 & 447.2591 & 2.5 \\
y7 & 716.3614 & 716.3594 & 2.8 \\
y8 & 831.3883 & 831.3853 & 3.6 \\
y9 & 960.4309 & 960.4294 & 1.6 \\
y10 & 1017.4524 & 1017.4504 & 1.9 \\
\hline
\end{tabular}


Figure S26. ThtA-T5A variant-modified products: HRMS/MS.

A) HRMS/MS of doubly modified product formed by ThtA-T5A variant. A. Fragmentation of the doubly modified product of ThtA-T5A variant annotated with observed b- and y-ions. Green denotes the substituted position. Black brackets connecting Thr and Asp indicate sites of macrolactone formation. B. Annotated CID spectrum of the doubly modified product of ThtA-T5A variant. C. Table of daughter ion assignments

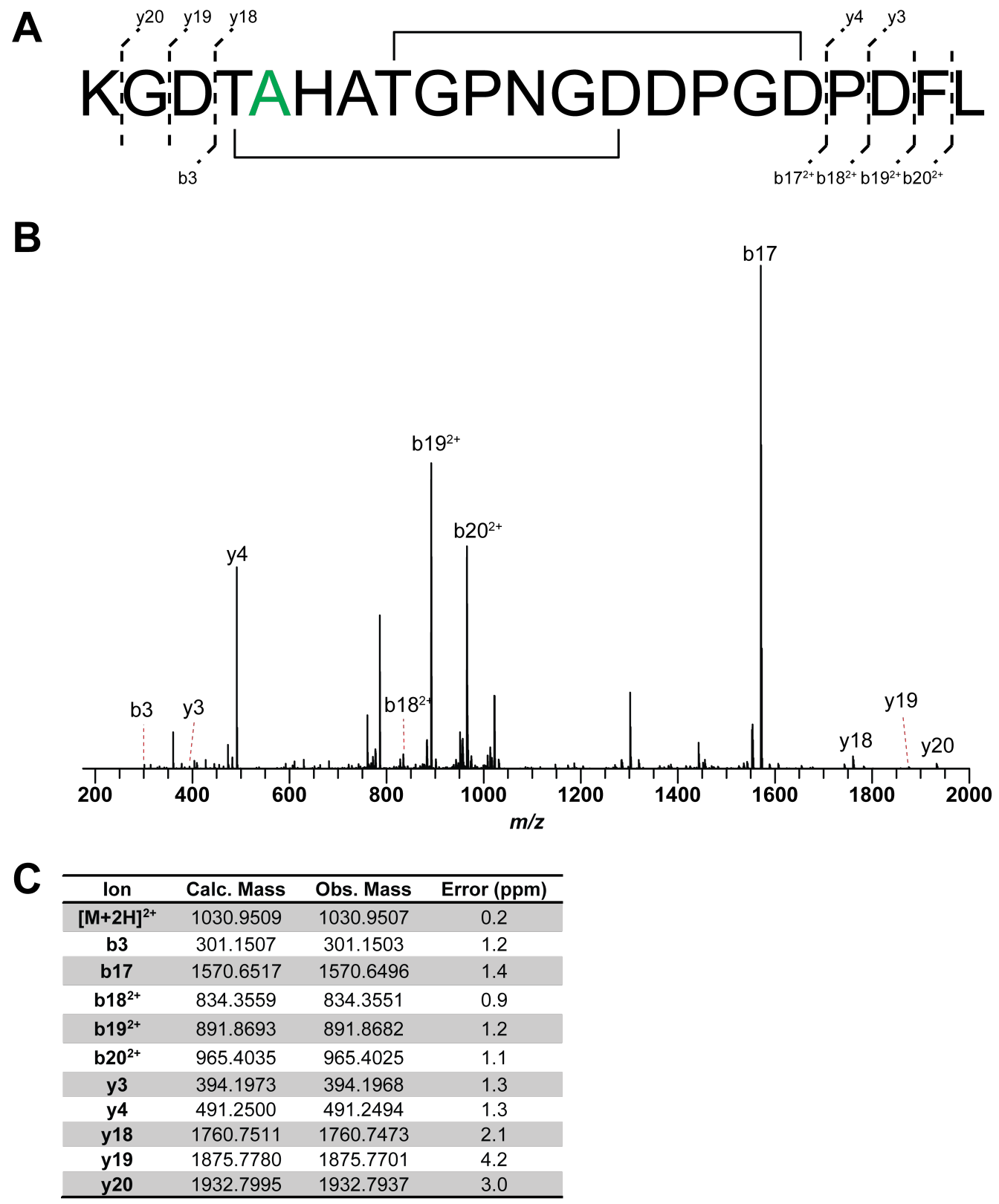


B) HRMS/MS of fully methanolized doubly modified product formed by ThtA-T5A variant. A. Fragmentation of the doubly modified product of ThtA-T5A variant after complete methanolysis. Green denotes the substituted position. Purple indicates an Asp methyl ester. Observed b- and $y$-ions are annotated. B. Annotated CID spectrum of the fully methanolized doubly modified product of ThtA-T5A variant. C. Table of daughter ion assignments

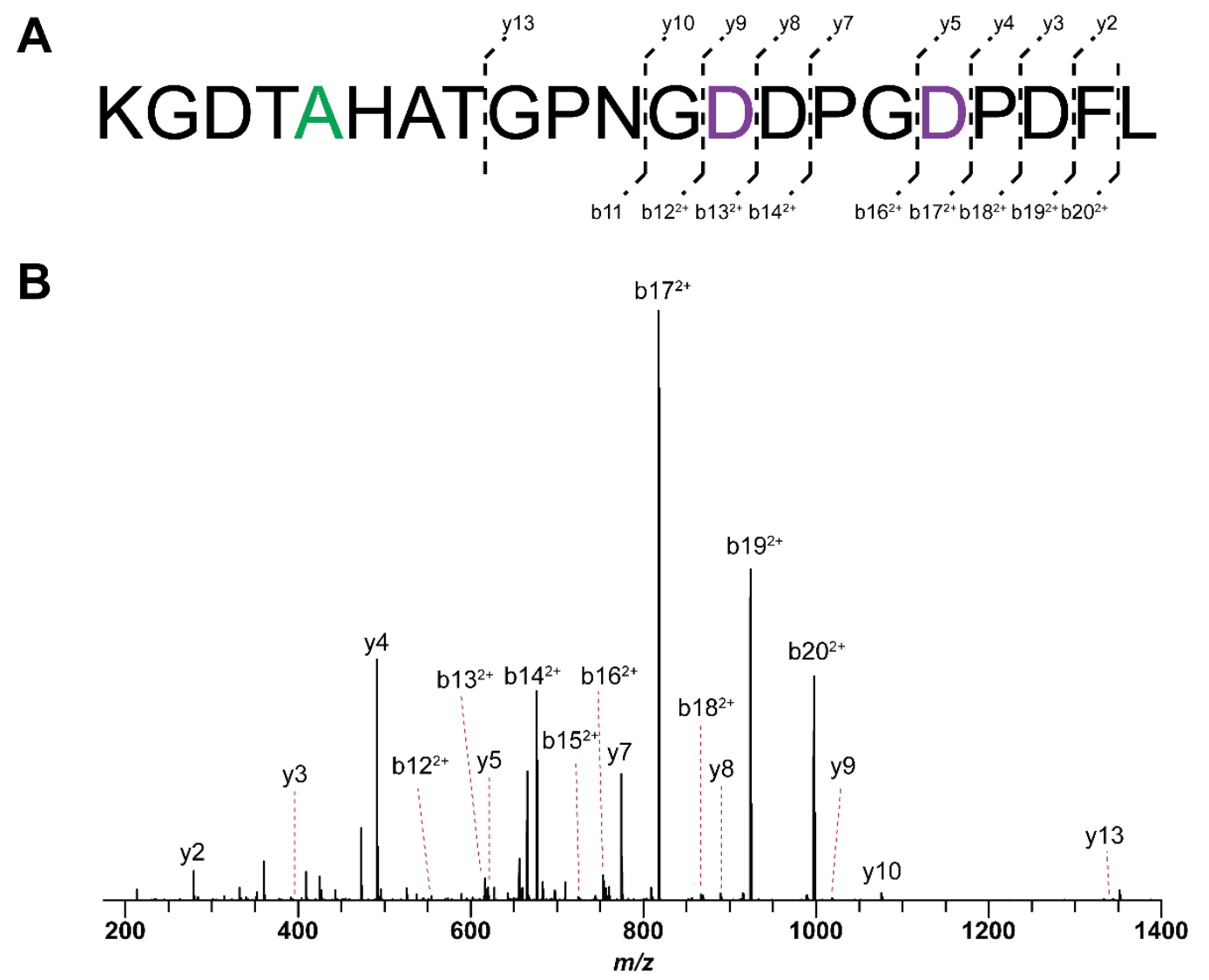

\begin{tabular}{|c|c|c|c|}
\hline Ion & Calc. Mass & Obs. Mass & Error (ppm) \\
\hline$[\mathrm{M}+3 \mathrm{H}]^{3+}$ & 708.9871 & 708.9864 & 1.0 \\
\hline b11 & 1050.4963 & 1050.4941 & 2.1 \\
\hline b12 $2^{2+}$ & 554.2625 & 554.2616 & 1.7 \\
\hline b13 ${ }^{2+}$ & 618.7838 & 618.7830 & 1.3 \\
\hline b14 ${ }^{2+}$ & 676.2973 & 676.2965 & 1.2 \\
\hline b15 $5^{2+}$ & 724.8237 & 724.8234 & 0.4 \\
\hline b16 ${ }^{2+}$ & 753.3344 & 753.3333 & 1.4 \\
\hline b17 $7^{2+}$ & 817.8557 & 817.8547 & 1.2 \\
\hline b18 ${ }^{2+}$ & 866.3821 & 866.3812 & 1.0 \\
\hline b19 ${ }^{2+}$ & 923.8956 & 923.8944 & 1.2 \\
\hline
\end{tabular}

\begin{tabular}{cccc}
\hline Ion & Calc. Mass & Obs. Mass & Error $(\mathbf{p p m})$ \\
\hline b20 $^{2+}$ & 997.4298 & 997.4283 & 1.5 \\
\hline y2 & 279.1703 & 279.1699 & 1.5 \\
y3 & 394.1973 & 394.1968 & 1.2 \\
y4 & 491.2500 & 491.2493 & 1.4 \\
y5 & 620.2926 & 620.2918 & 1.4 \\
y7 & 774.3669 & 774.3658 & 1.3 \\
y8 & 889.3938 & 889.3928 & 1.1 \\
y9 & 1018.4364 & 1018.4349 & 1.5 \\
\hline y10 & 1075.4579 & 1075.4564 & 1.4 \\
\hline y13 & 1343.5750 & 1343.5734 & 1.2 \\
\hline
\end{tabular}


C) HRMS/MS of partially methanolized doubly modified product formed by ThtA-T5A variant. A. Fragmentation of the partially methanolized doubly modified product of ThtA-T5A variant. Observed $b$ - and $y$-ions are annotated. Green denotes the substituted position. Purple indicates an Asp methyl ester. The black bracket connecting Thr and Asp indicates the site of macrolactone formation. B. Annotated CID spectrum of the partially methanolized doubly modified product of ThtA-T5A variant. C. Table of daughter ion assignments.

A

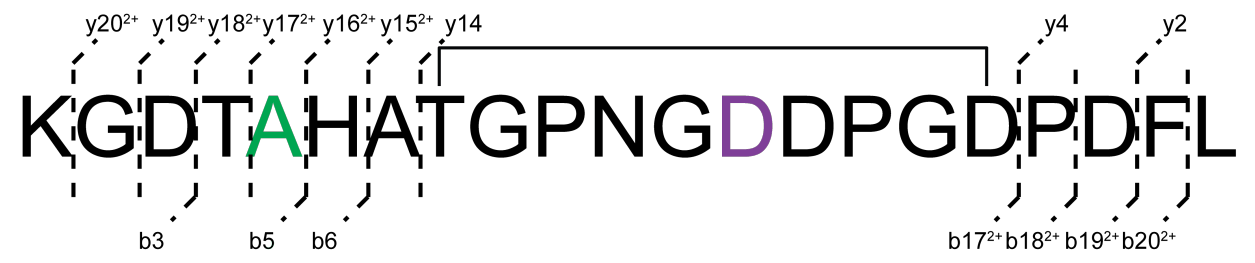

B

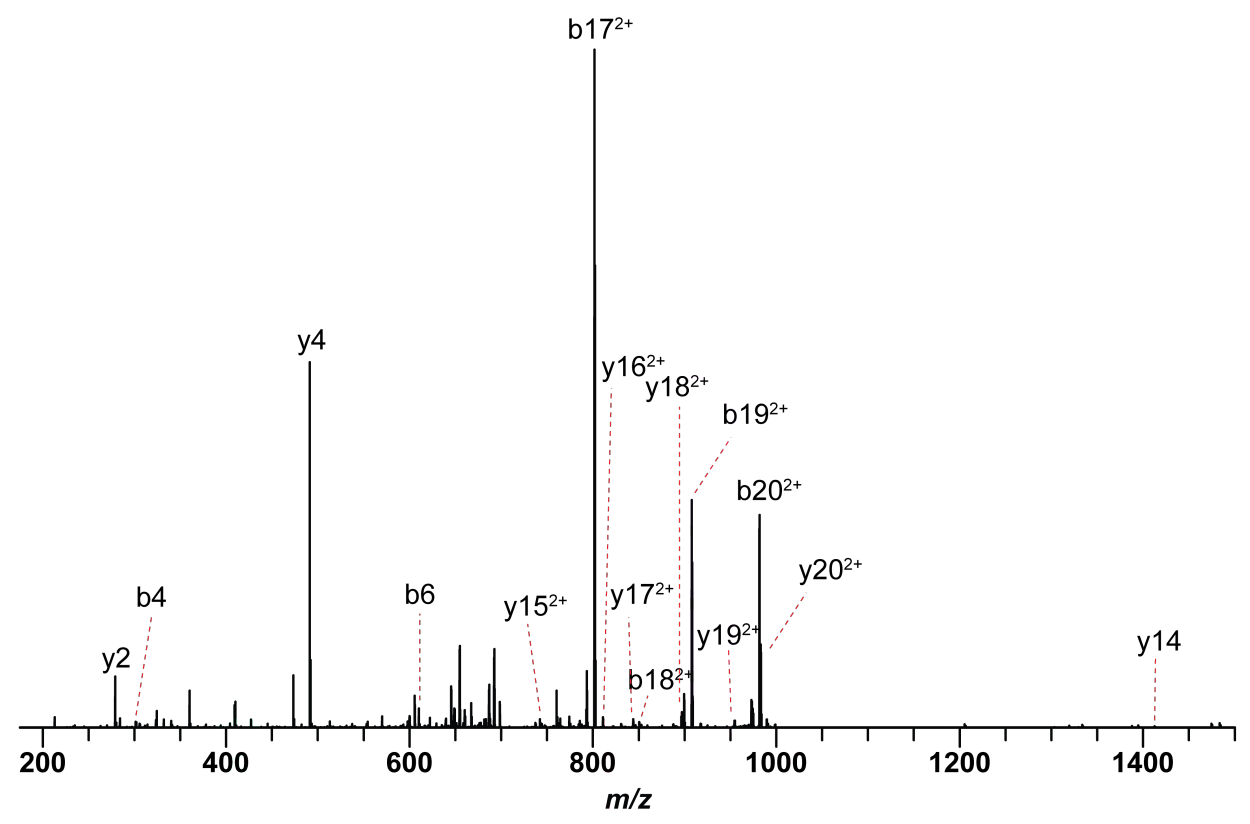

C

\begin{tabular}{cccc}
\hline Ion & Calc. Mass & Obs. Mass & Error (ppm) \\
\hline${\text { [M+3H }]^{{ }^{+}}}^{\text {b }}$ & 698.3117 & 698.3112 & 0.8 \\
\hline b3 & 301.1507 & 301.1502 & 1.5 \\
b6 & 610.2944 & 610.2934 & 1.6 \\
b17 $^{2+}$ & 801.8426 & 801.8414 & 1.5 \\
b18 $^{2+}$ & 850.3690 & 850.3682 & 0.9 \\
b19 $^{2+}$ & 907.8824 & 907.8809 & 1.7 \\
b20 $^{2+}$ & 981.4166 & 981.4150 & 1.7 \\
y2 & 279.1703 & 279.1699 & 1.5 \\
y4 & 491.2500 & 491.2494 & 1.3 \\
y14 & 1412.5965 & 1412.5934 & 2.2 \\
\hline
\end{tabular}

\begin{tabular}{cccc}
\hline Ion & Calc. Mass & Obs. Mass & Error (ppm) \\
\hline $\mathbf{y 1 5 ^ { 2 + }}$ & 742.3204 & 742.3192 & 1.7 \\
$\mathbf{y 1 6}^{2+}$ & 810.8499 & 810.8481 & 2.2 \\
$\mathbf{y} 17^{2+}$ & 846.3684 & 846.3669 & 1.8 \\
$\mathbf{y 1 8 ^ { 2 + }}$ & 896.8923 & 896.8907 & 1.8 \\
$\mathbf{y 1 9}^{2+}$ & 954.4058 & 954.4042 & 1.6 \\
$\mathbf{y 2 0}{ }^{2+}$ & 982.9165 & 982.9146 & 1.9 \\
\hline
\end{tabular}


D) HRMS/MS of singly modified product-1 formed by ThtA-T5A variant. A. Fragmentation of singly modified product-1 formed upon co-expression of ThtA-T5A variant with ThtB. Observed b- and $y$-ions are annotated. Green denotes the substituted position. The black bracket connecting Thr and Asp indicates the site of macrolactone formation. B. Annotated CID spectrum of singly modified product-1. C. Table of daughter ion assignments.

A

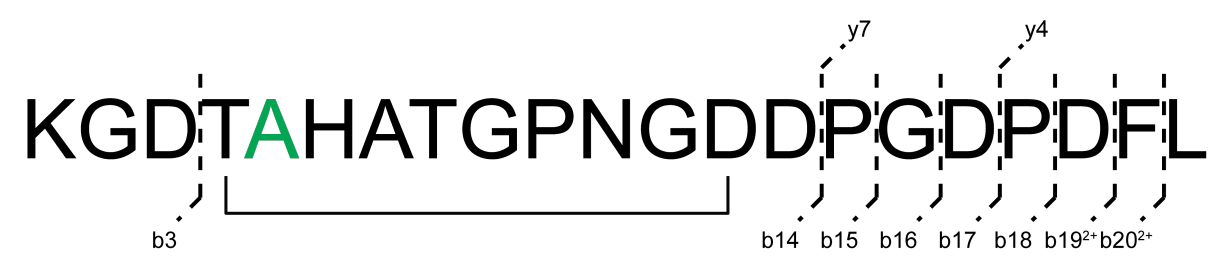

B

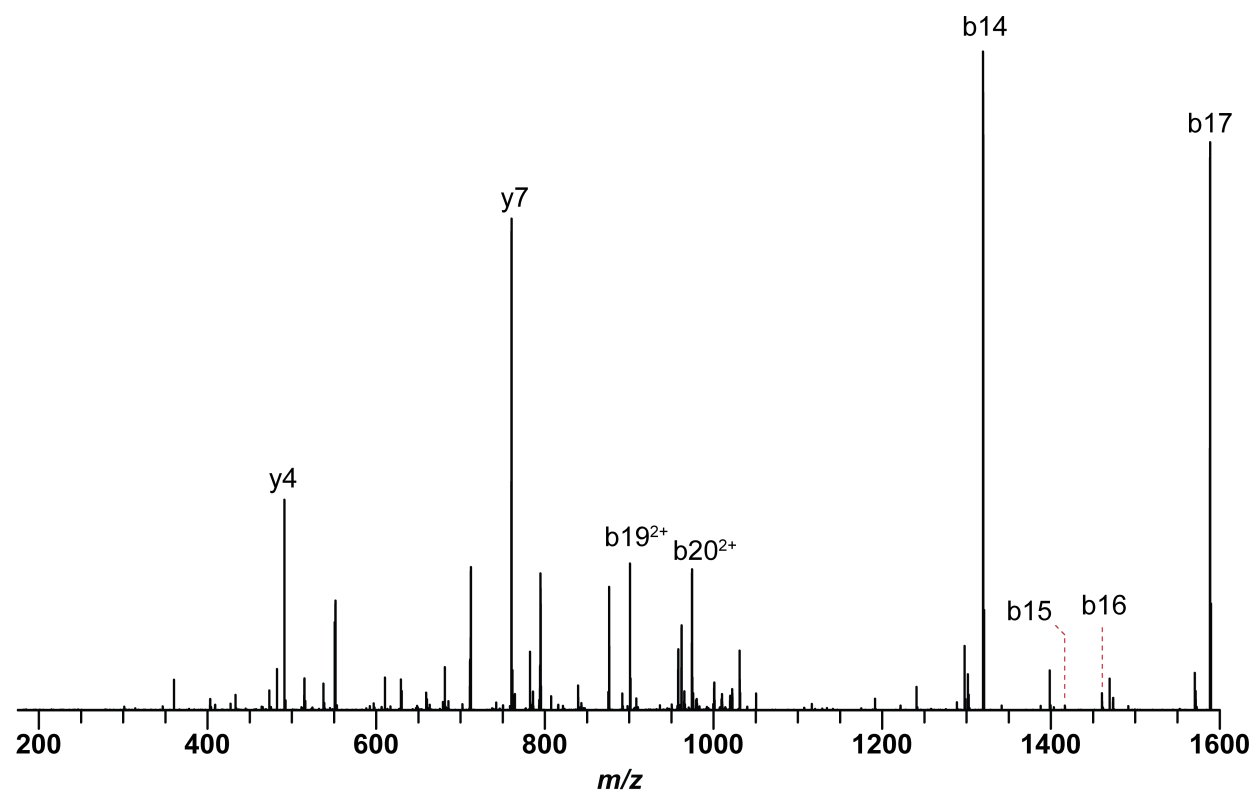

C

\begin{tabular}{cccc}
\hline Ion & Calc. Mass & Obs. Mass & Error $(\mathbf{p p m})$ \\
\hline${\text { [M+2H }]^{2+}}^{\mathbf{M}}$ & 1039.9561 & 1039.9554 & 0.7 \\
$\mathbf{b 1 4}$ & 1319.5611 & 1319.5586 & 1.9 \\
$\mathbf{b 1 5}$ & 1416.6139 & 1416.6134 & 0.3 \\
\hline b16 & 1473.6353 & 1473.6325 & 1.9 \\
\hline b17 & 1588.6623 & 1588.6600 & 1.4 \\
b19 $^{2+}$ & 900.8746 & 900.8729 & 1.9 \\
b20 $^{2+}$ & 974.4088 & 974.4074 & 1.5 \\
\hline y4 & 491.2500 & 491.2494 & 1.2 \\
\hline y7 & 760.3512 & 760.3500 & 1.5 \\
\hline
\end{tabular}


E) HRMS/MS of methanolized singly modified product-1 formed by ThtA-T5A variant. A. Fragmentation of methanolized singly modified product-1 formed upon co-expression of ThtAT5A variant with ThtB. Observed b- and y-ions are annotated. Green denotes the substituted position. Purple denotes an Asp methyl ester. B. Annotated CID spectrum of fully methanolized singly modified product-1. C. Table of daughter ion assignments.

A

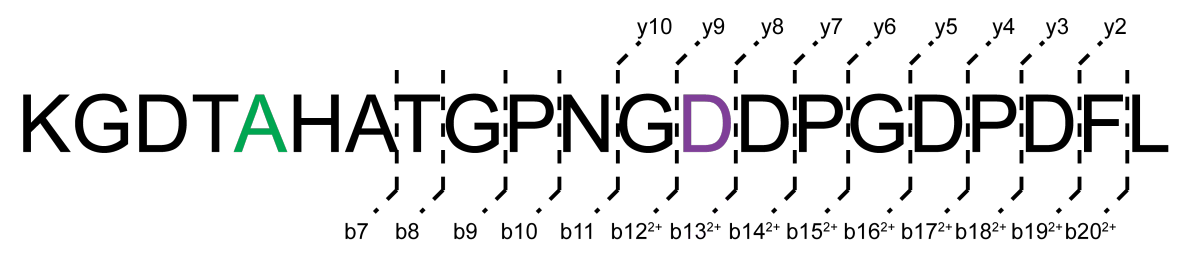

B

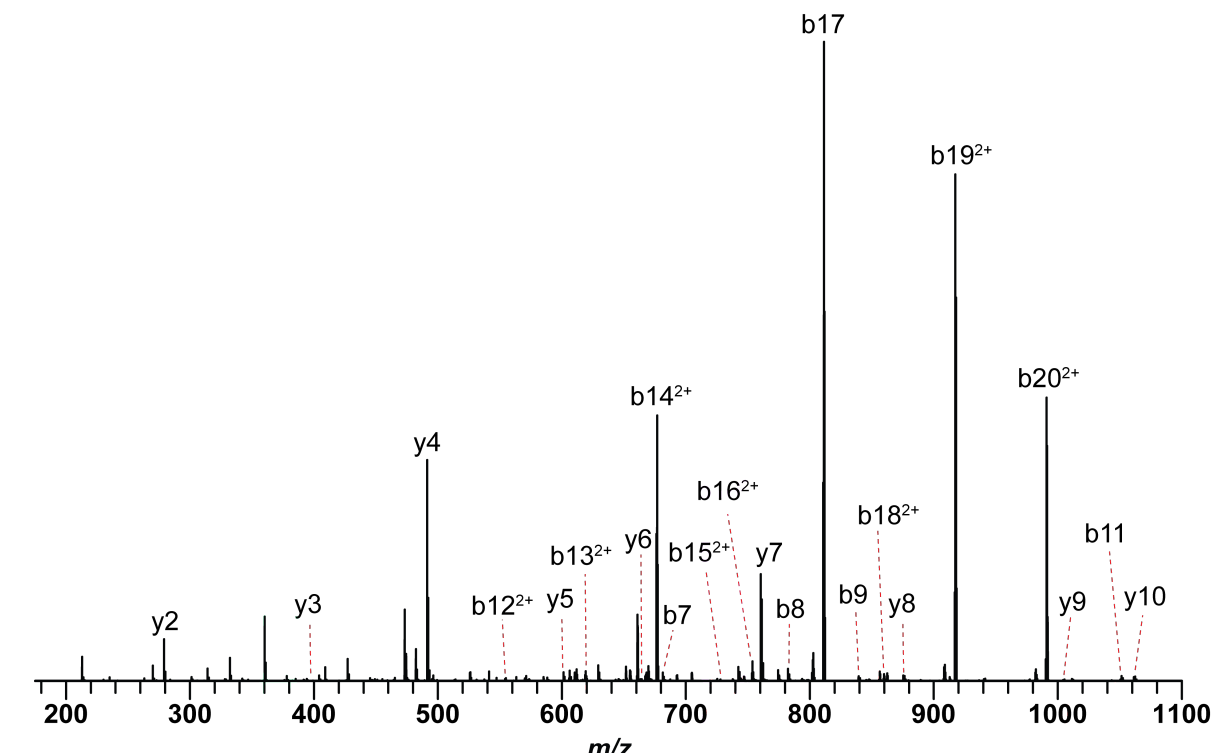

C

\begin{tabular}{cccc}
\hline Ion & Calc. Mass & Obs. Mass & Error $(\mathbf{p p m})$ \\
\hline${\text { [M+3H }]^{3+}}^{\text {b7 }}$ & 704.3153 & 704.3148 & 0.7 \\
b7 & 681.3315 & 681.3309 & 0.8 \\
b8 & 782.3791 & 782.3785 & 0.8 \\
b9 & 839.4006 & 839.4000 & 0.7 \\
b10 & 936.4534 & 936.4533 & 0.0 \\
b11 & 1050.4963 & 1050.4949 & 1.3 \\
b12 $^{2+}$ & 554.2625 & 554.2620 & 1.0 \\
b13 $^{2+}$ & 618.7838 & 618.7833 & 0.8 \\
b14 $^{2+}$ & 676.2973 & 676.2968 & 0.7 \\
b15 $^{2+}$ & 724.8237 & 724.8229 & 1.1 \\
b16 $^{2+}$ & 753.3344 & 753.3337 & 1.0 \\
b17 $^{2+}$ & 810.8479 & 810.8473 & 0.7 \\
b18 $^{2+}$ & 859.3743 & 859.3741 & 0.1 \\
b19 $^{2+}$ & 916.8877 & 916.8871 & 0.7 \\
b20 $^{2+}$ & 990.4219 & 990.4213 & 0.6 \\
\hline
\end{tabular}

\begin{tabular}{cccc}
\hline lon & Calc. Mass & Obs. Mass & Error $(\mathbf{p p m})$ \\
\hline y2 & 279.1703 & 279.1699 & 1.4 \\
y3 & 394.1973 & 394.1970 & 0.8 \\
y4 & 491.2500 & 491.2496 & 1.0 \\
y5 & 606.2770 & 606.2765 & 0.7 \\
y6 & 663.2984 & 663.2976 & 1.3 \\
y7 & 760.3512 & 760.3506 & 0.8 \\
y8 & 875.3781 & 875.3778 & 0.4 \\
y9 & 1004.4207 & 1004.4205 & 0.2 \\
y10 & 1061.4422 & 1061.4410 & 1.1 \\
\hline
\end{tabular}


F) HRMS/MS of singly modified product-2 formed by ThtA-T5A variant. A. Fragmentation of singly modified product-2 formed upon co-expression of ThtA-T5A variant with ThtB. Observed b- and y-ions are annotated. Green denotes the substituted position. The black bracket connecting Thr and Asp indicates the site of macrolactone formation. B. Annotated CID spectrum of singly modified product-2. C. Table of daughter ion assignments.

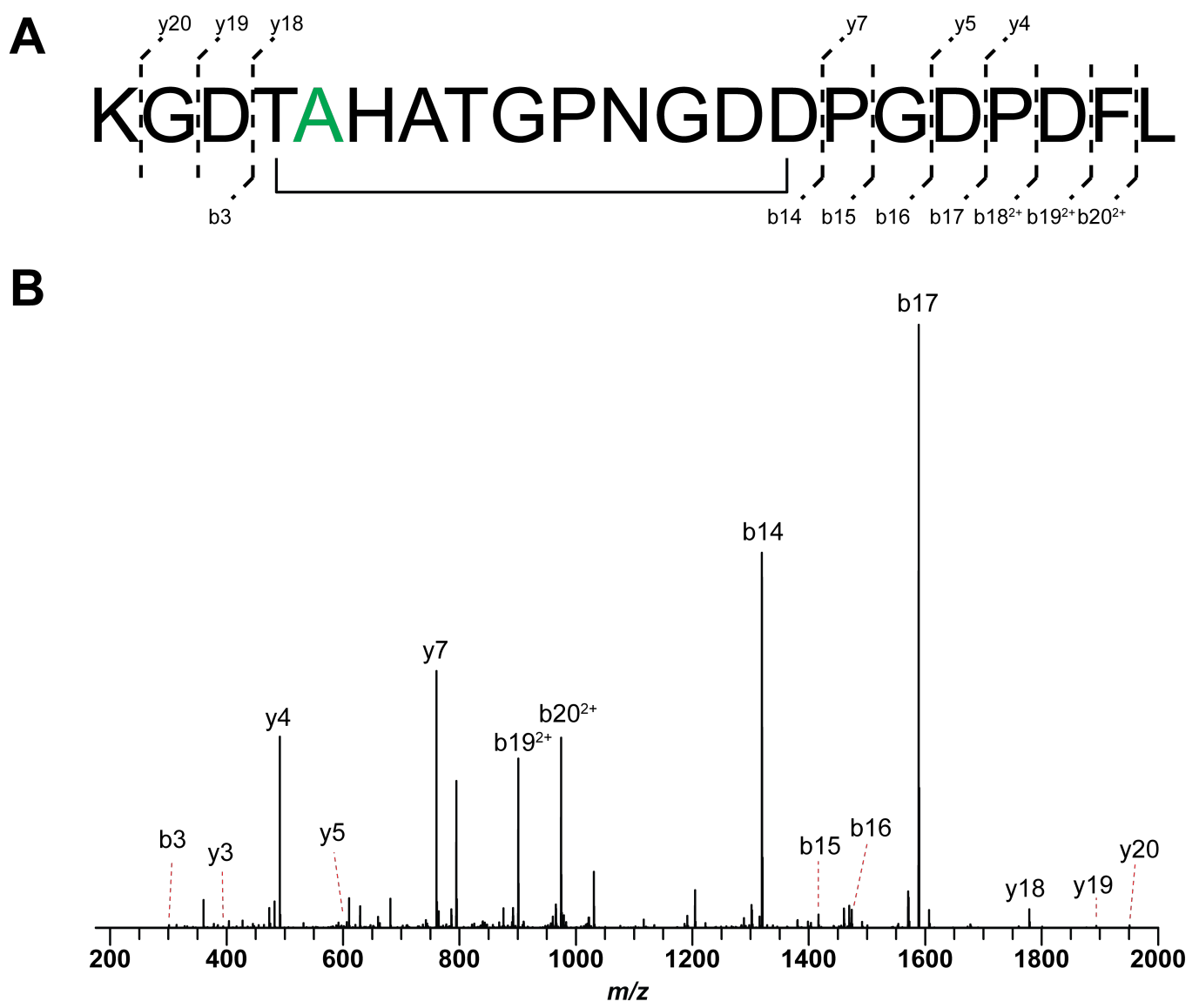

\begin{tabular}{cccc}
\hline Ion & Calc. Mass & Obs. Mass & Error $(\mathbf{p p m})$ \\
\hline$[\mathbf{M + 2 H}]^{2+}$ & 1039.9561 & 1039.9551 & 1.0 \\
\hline b3 & 301.1507 & 301.1502 & 1.4 \\
\hline b14 & 1319.5611 & 1319.5581 & 2.3 \\
\hline b15 & 1416.6139 & 1416.6115 & 1.7 \\
\hline b16 & 1473.6353 & 1473.6327 & 1.8 \\
\hline b17 & 1588.6623 & 1588.6593 & 1.9 \\
\hline b18 $^{2+}$ & 843.3611 & 843.3602 & 1.1 \\
\hline b19 $^{2+}$ & 900.8746 & 900.8730 & 1.8 \\
\hline b20 $^{2+}$ & 974.4088 & 974.4071 & 1.8 \\
\hline y3 $^{2}$ & 394.1973 & 394.1968 & 1.2 \\
\hline
\end{tabular}

\begin{tabular}{cccc}
\hline Ion & Calc. Mass & Obs. Mass & Error (ppm) \\
\hline y4 & 491.2500 & 491.2493 & 1.4 \\
y5 & 606.2770 & 606.2760 & 1.6 \\
y7 & 760.3512 & 760.3499 & 1.8 \\
y18 & 1778.7616 & 1778.7574 & 2.4 \\
y19 & 1893.7886 & 1893.7837 & 2.6 \\
y20 & 1950.8100 & 1950.8038 & 3.2 \\
\hline
\end{tabular}


G) HRMS/MS of methanolized singly modified product-2 formed by ThtA-T5A variant. A. Fragmentation of methanolized singly modified product-2 formed upon co-expression of ThtAT5A variant with ThtB. Observed b- and y-ions are annotated. Green denotes the substituted position. Purple refers to an Asp methyl ester. B. Annotated CID spectrum of fully methanolized singly modified product-2. C. Table of daughter ion assignments.

A

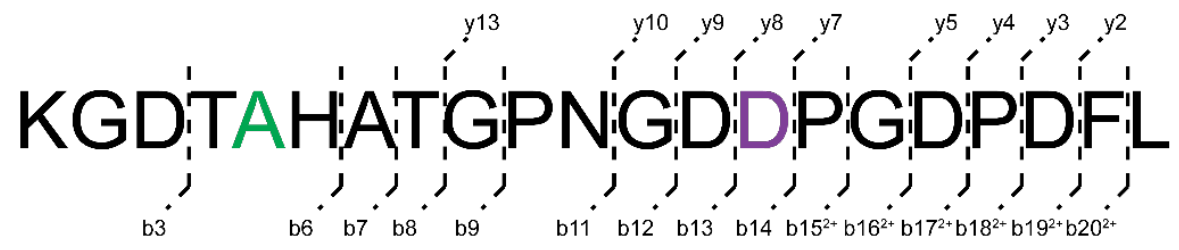

B

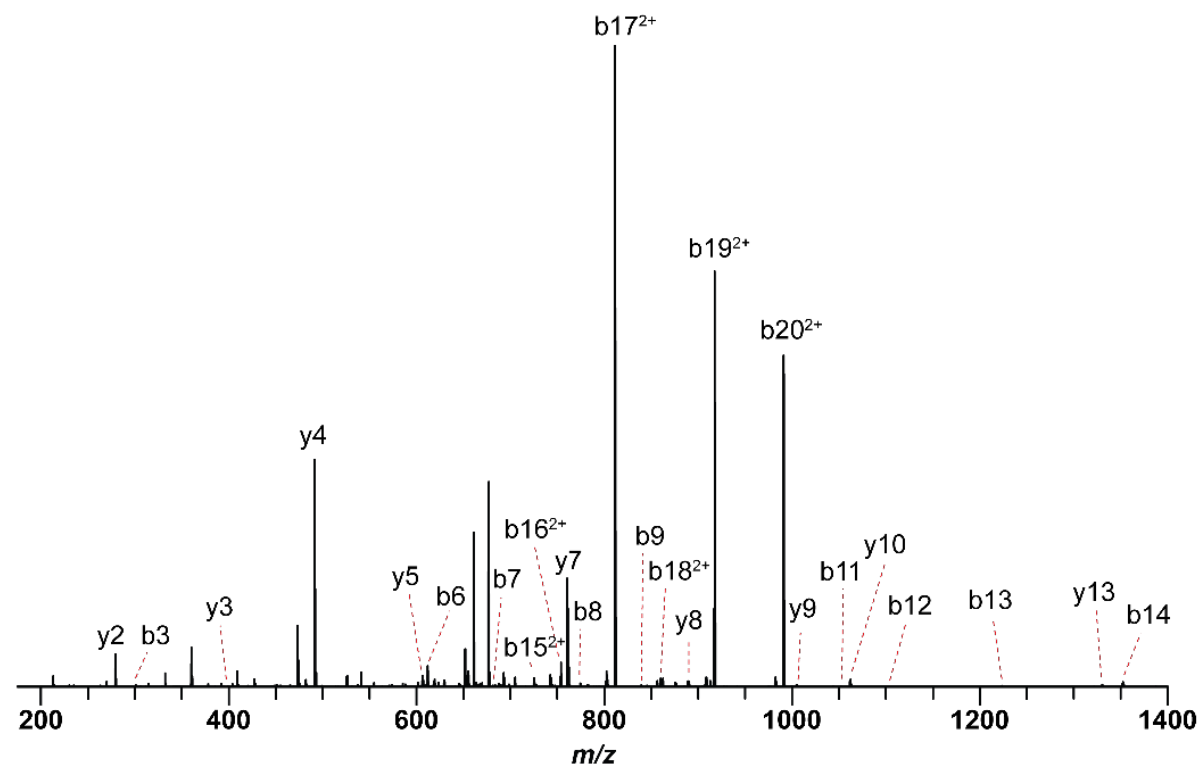

C

\begin{tabular}{|cccc|}
\hline Ion & Calc. Mass & Obs. Mass & Error (ppm) \\
\hline${\text { [M+3H }]^{3+}}^{+}$ & 704.3153 & 704.3147 & 0.8 \\
\hline b3 & 301.1507 & 301.1501 & 1.7 \\
\hline b6 & 610.2944 & 610.2935 & 1.4 \\
\hline b7 & 681.3315 & 681.3306 & 1.2 \\
\hline b8 & 782.3791 & 782.3782 & 1.3 \\
\hline b9 & 839.4006 & 839.3998 & 0.9 \\
\hline b11 & 1050.4963 & 1050.4953 & 1.0 \\
\hline b12 & 1107.5178 & 1107.5184 & 0.5 \\
\hline b13 & 1222.5447 & 1222.5430 & 1.4 \\
\hline b14 & 1351.5873 & 1351.5858 & 1.1 \\
\hline b15 $^{2+}$ & 724.8237 & 724.8227 & 1.4 \\
\hline b16 $^{2+}$ & 753.3344 & 753.3334 & 1.4 \\
\hline b17 $^{2+}$ & 810.8479 & 810.8469 & 1.2 \\
\hline b18 $^{2+}$ & 859.3743 & 859.3735 & 0.9 \\
\hline b19 $^{2+}$ & 916.8877 & 916.8868 & 1.0 \\
\hline
\end{tabular}

\begin{tabular}{cccc}
\hline Ion & Calc. Mass & Obs. Mass & Error $(\mathbf{p p m})$ \\
\hline b20 ${ }^{2+}$ & 990.4219 & 990.4209 & 1.0 \\
\hline y2 & 279.1703 & 279.1698 & 1.7 \\
y3 & 394.1973 & 394.1969 & 1.0 \\
y4 & 491.2500 & 491.2493 & 1.5 \\
y5 & 606.2770 & 606.2763 & 1.2 \\
y7 & 760.3512 & 760.3502 & 1.4 \\
y8 & 889.3938 & 889.3927 & 1.2 \\
y9 & 1004.4207 & 1004.4197 & 1.1 \\
y10 & 1061.4422 & 1061.4410 & 1.1 \\
y13 & 1329.5594 & 1329.5585 & 0.7 \\
\hline
\end{tabular}


H) HRMS/MS of singly modified product-3 formed by ThtA-T5A variant. A. Fragmentation of singly modified product-3 formed upon co-expression of ThtA-T5A variant with ThtB. Observed b- and $y$-ions are annotated. Green denotes the substituted position. The black bracket connecting Thr and Asp indicates the site of macrolactone formation. B. Annotated CID spectrum of singly modified product-3. C. Table of daughter ion assignments.

A

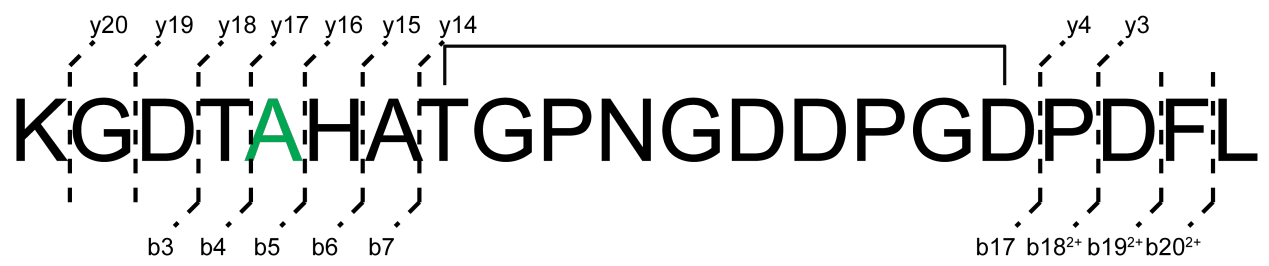

B

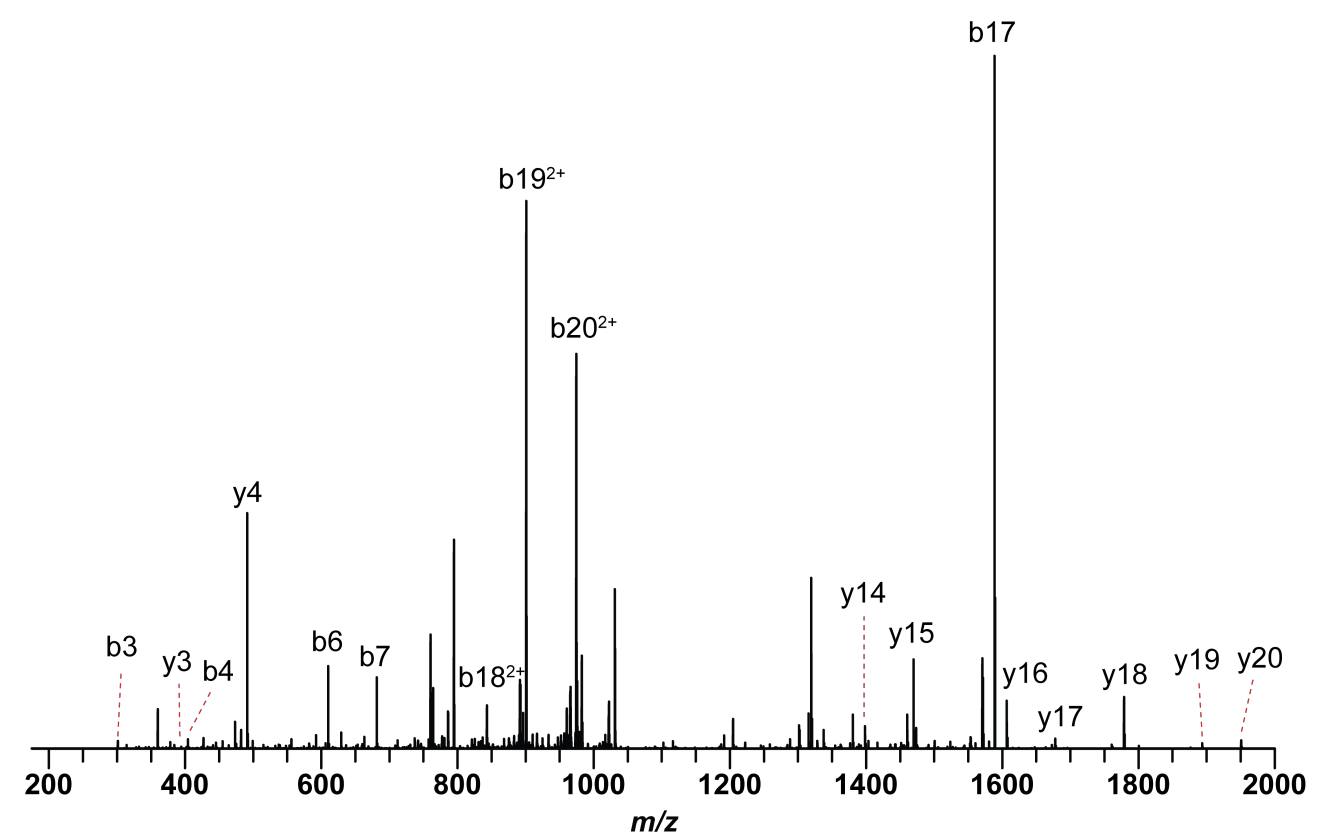

C

\begin{tabular}{cccc}
\hline lon & Calc. Mass & Obs. Mass & Error $(\mathbf{p p m})$ \\
\hline$[\mathbf{M + 2 H}]^{2+}$ & 1039.9561 & 1039.9552 & 0.9 \\
b3 & 301.1507 & 301.1502 & 1.5 \\
b4 & 402.1983 & 402.1979 & 0.9 \\
b6 & 610.2944 & 610.2936 & 1.2 \\
b7 & 681.3315 & 681.3308 & 1.0 \\
b17 & 1588.6623 & 1588.6602 & 1.3 \\
b18 $^{2+}$ & 843.3611 & 843.3605 & 0.8 \\
b19 $^{2+}$ & 900.8746 & 900.8736 & 1.2 \\
b20 $^{2+}$ & 974.4088 & 974.4077 & 1.1 \\
\hline y3 & 394.1973 & 394.1970 & 0.7 \\
\hline
\end{tabular}

\begin{tabular}{cccc}
\hline Ion & Calc. Mass & Obs. Mass & Error $(\mathbf{p p m})$ \\
\hline y4 & 491.2500 & 491.2495 & 1.2 \\
\hline y14 & 1398.5808 & 1398.5797 & 0.8 \\
y15 & 1469.6179 & 1469.6163 & 1.1 \\
\hline y16 & 1606.6768 & 1606.6746 & 1.4 \\
y17 & 1677.7140 & 1677.7119 & 1.2 \\
y18 & 1778.7616 & 1778.7583 & 1.9 \\
\hline y19 & 1893.7886 & 1893.7857 & 1.5 \\
\hline y20 & 1950.8100 & 1950.8060 & 2.1 \\
\hline
\end{tabular}


I) HRMS/MS of methanolized singly modified product-3 formed by ThtA-T5A variant. A. Fragmentation of methanolized singly modified product-3 formed upon co-expression of ThtAT5A variant with ThtB. Observed b- and y-ions are annotated. Green denotes the substituted position. Purple denotes an Asp methyl ester. B. Annotated CID spectrum of fully methanolized singly modified product-3. C. Table of daughter ion assignments.

A

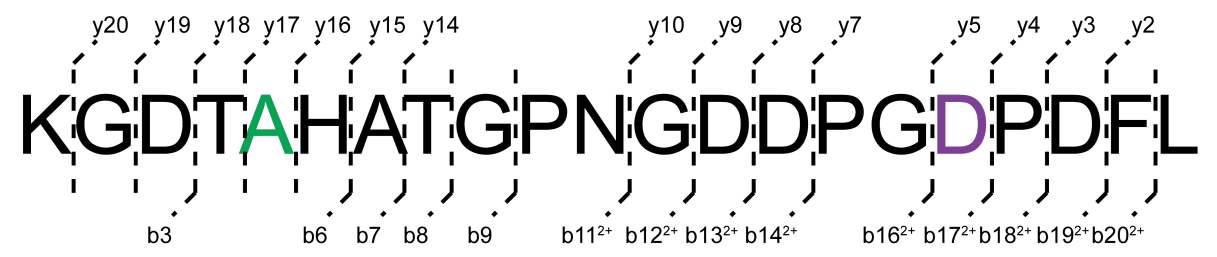

B

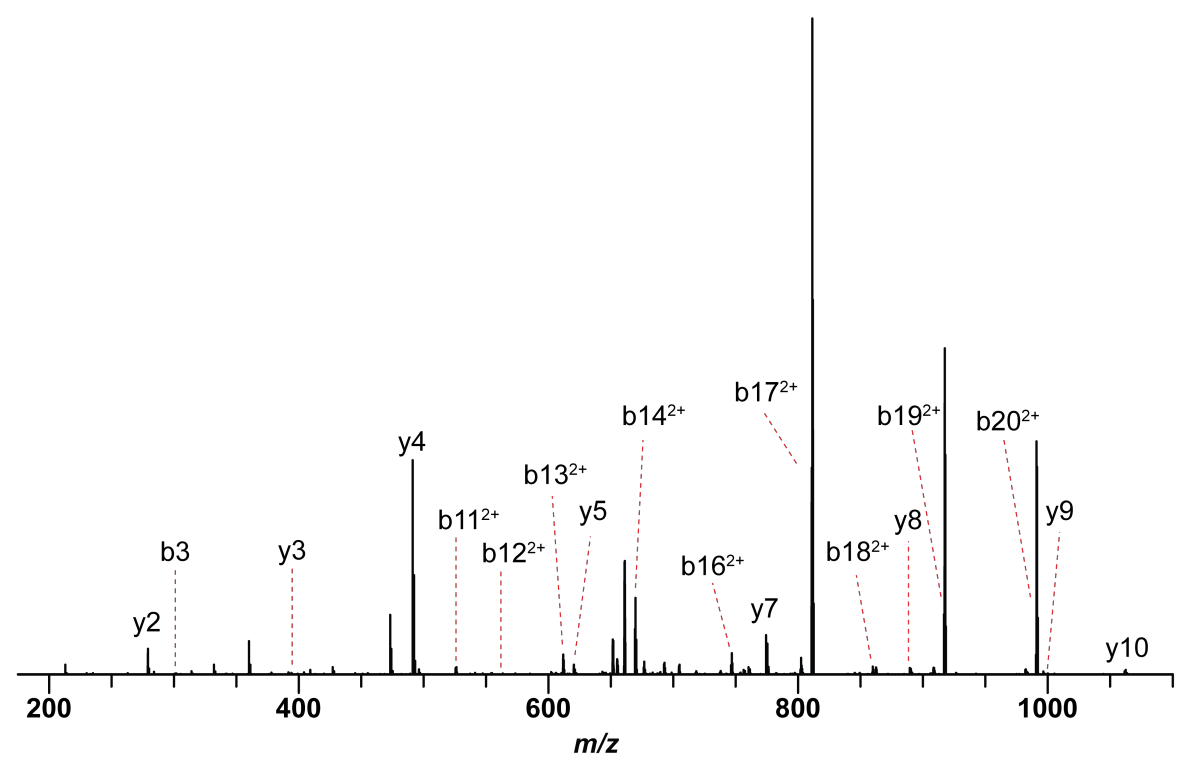

\begin{tabular}{cccc}
$\mathbf{C}$ & \multicolumn{3}{c}{} \\
\hline Ion & Calc. Mass & Obs. Mass & Error $(\mathbf{p p m})$ \\
\hline$[\mathbf{M + 3 H}]^{3+}$ & 704.3153 & 704.3148 & 0.7 \\
b3 & 301.1507 & 301.1502 & 1.4 \\
\hline b6 & 610.2944 & 610.2937 & 1.1 \\
b7 & 681.3315 & 681.3305 & 1.5 \\
\hline b8 & 782.3791 & 782.3781 & 1.4 \\
\hline b9 & 839.4006 & 839.3993 & 1.6 \\
\hline b11 ${ }^{2+}$ & 525.7518 & 525.7511 & 1.3 \\
\hline b12 $^{2+}$ & 554.2625 & 554.2616 & 1.7 \\
\hline b13 $^{2+}$ & 611.7760 & 611.7753 & 1.2 \\
\hline b14 $^{2+}$ & 669.2895 & 669.2887 & 1.2 \\
\hline b16 $^{2+}$ & 746.3266 & 746.3255 & 1.5 \\
\hline b17 $^{2+}$ & 810.8479 & 810.8469 & 1.2 \\
\hline
\end{tabular}

\begin{tabular}{cccc}
\hline Ion & Calc. Mass & Obs. Mass & Error $(\mathbf{p p m})$ \\
\hline b18 $^{2+}$ & 859.3743 & 859.3736 & 0.7 \\
b19 $^{2+}$ & 916.8877 & 916.8867 & 1.1 \\
b20 $^{2+}$ & 990.4219 & 990.4208 & 1.1 \\
\hline y2 & 279.1703 & 279.1699 & 1.6 \\
y3 & 394.1973 & 394.1967 & 1.4 \\
y4 & 491.2500 & 491.2493 & 1.4 \\
y5 & 620.2926 & 620.2918 & 1.4 \\
y7 & 774.3669 & 774.3658 & 1.3 \\
y8 & 889.3938 & 889.3928 & 1.1 \\
\hline y9 & 1004.4207 & 1004.4206 & 0.1 \\
y10 & 1061.4422 & 1061.4407 & 1.4 \\
\hline
\end{tabular}


Figure S27. ThtA-T8A variant-modified products: HRMS/MS.

A) HRMS/MS of singly modified product-1 formed by ThtA-T8A variant. A. Fragmentation of singly modified product-1 formed upon co-expression of ThtA-T8A variant with ThtB. Observed b- and y-ions are annotated. Green denotes the substituted position. The black bracket connecting Thr and Asp indicates the site of macrolactone formation. B. Annotated CID spectrum of singly modified product-1. C. Table of daughter ion assignments.

A

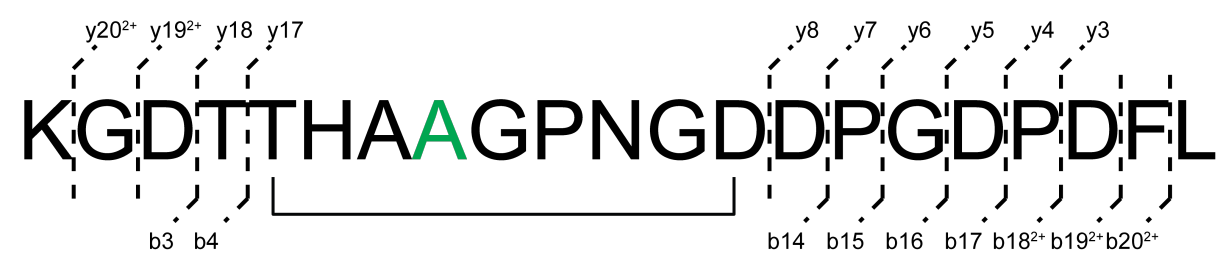

B

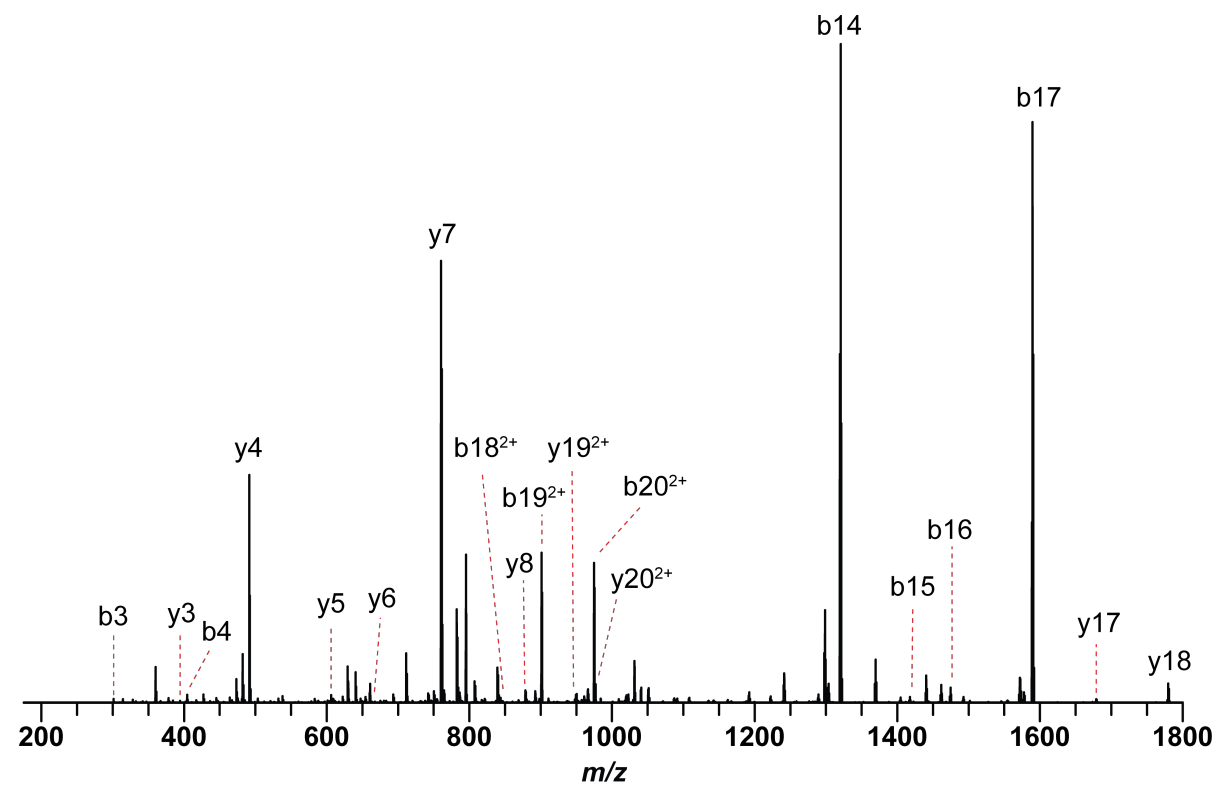

C

\begin{tabular}{|c|c|c|c|}
\hline Ion & Calc. Mass & Obs. Mass & Error (ppm) \\
\hline$[\mathrm{M}+2 \mathrm{H}]^{2+}$ & 1039.9561 & 1039.9555 & 0.6 \\
\hline b3 & 301.1507 & 301.1503 & 1.3 \\
\hline b4 & 402.1983 & 402.1978 & 1.4 \\
\hline b14 & 1319.5611 & 1319.5592 & 1.4 \\
\hline b15 & 1416.6139 & 1416.6124 & 1.0 \\
\hline b16 & 1473.6353 & 1473.6338 & 1.0 \\
\hline b17 & 1588.6623 & 1588.6602 & 1.3 \\
\hline b18 ${ }^{2+}$ & 843.3611 & 843.3616 & 0.6 \\
\hline b19 ${ }^{2+}$ & 900.8746 & 900.8736 & 1.1 \\
\hline b20 ${ }^{2+}$ & 974.4088 & 974.4078 & 1.1 \\
\hline
\end{tabular}

\begin{tabular}{cccc}
\hline Ion & Calc. Mass & Obs. Mass & Error (ppm) \\
\hline y3 & 394.1973 & 394.1969 & 1.0 \\
y4 & 491.2500 & 491.2494 & 1.4 \\
y5 & 606.2770 & 606.2763 & 1.1 \\
y6 & 663.2984 & 663.2975 & 1.4 \\
y7 & 760.3512 & 760.3504 & 1.1 \\
y8 & 875.3781 & 875.3778 & 0.4 \\
y17 & 1677.7140 & 1677.7127 & 0.8 \\
y18 & 1778.7616 & 1778.7585 & 1.8 \\
y19 $^{2+}$ & 947.3979 & 947.3959 & 2.2 \\
y20 $^{2+}$ & 975.9087 & 975.9112 & 2.6 \\
\hline
\end{tabular}


B) MS/MS of methanolized singly modified product-1 formed by ThtA-T8A variant. A. Fragmentation of methanolized singly modified product-1 formed upon co-expression of ThtAT8A variant with ThtB. Observed $b$ - and $y$-ions are annotated. Green denotes the substituted position. Purple denotes an Asp methyl ester. B. Annotated CID spectrum of fully methanolized singly modified product-1. C. Table of daughter ion assignments.

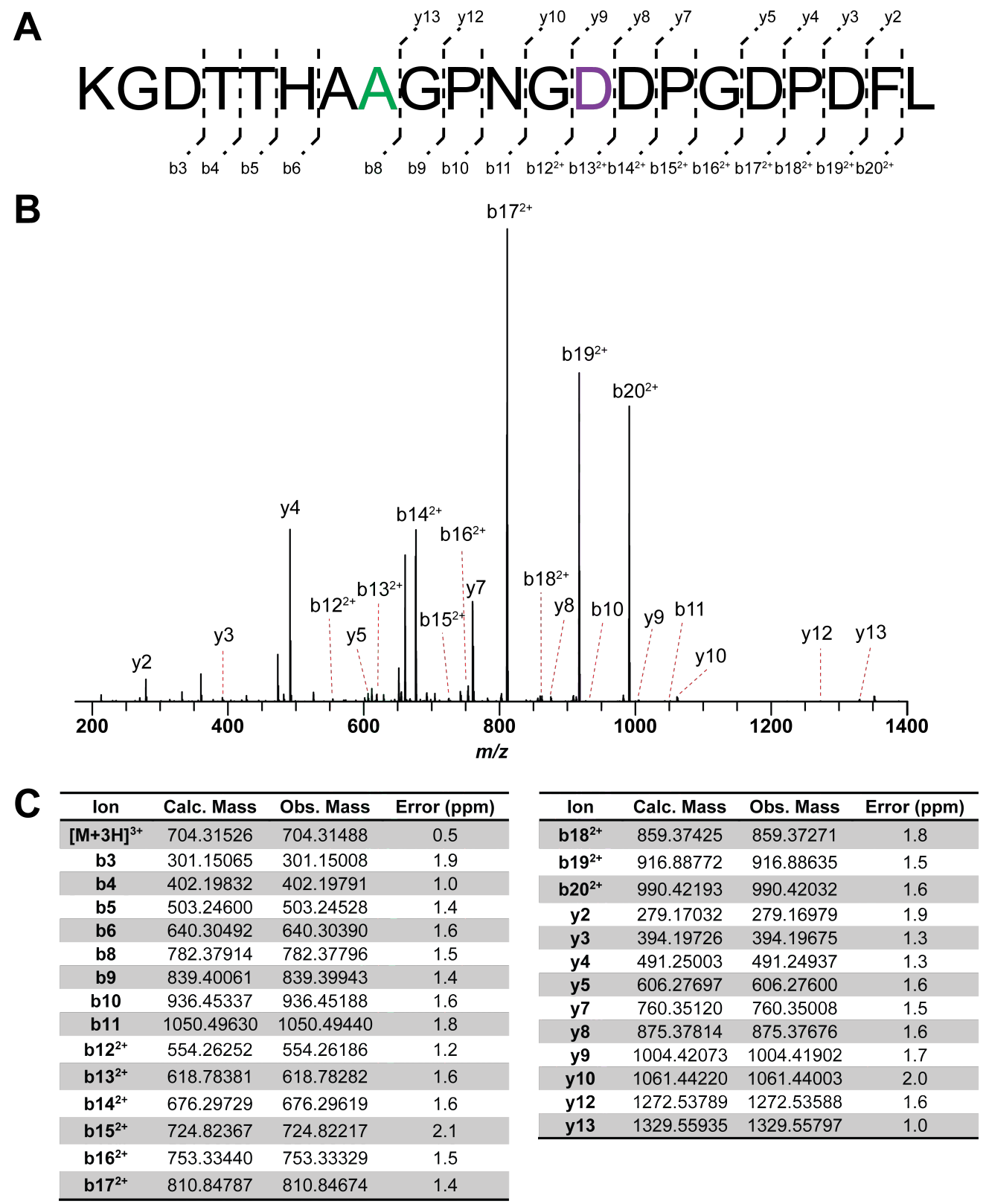


C) HRMS/MS of singly modified product-2 formed by ThtA-T8A variant. A. Fragmentation of singly modified product-2 formed upon co-expression of ThtA-T8A variant with ThtB. Observed b- and $y$-ions are annotated. Green denotes the substituted position. The black bracket connecting Thr and Asp indicates the site of macrolactone formation. B. Annotated CID spectrum of singly modified product-2. C. Table of daughter ion assignments.

A

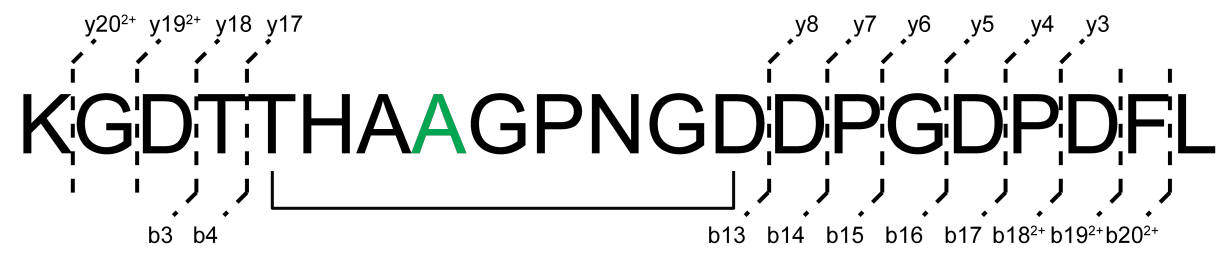

B

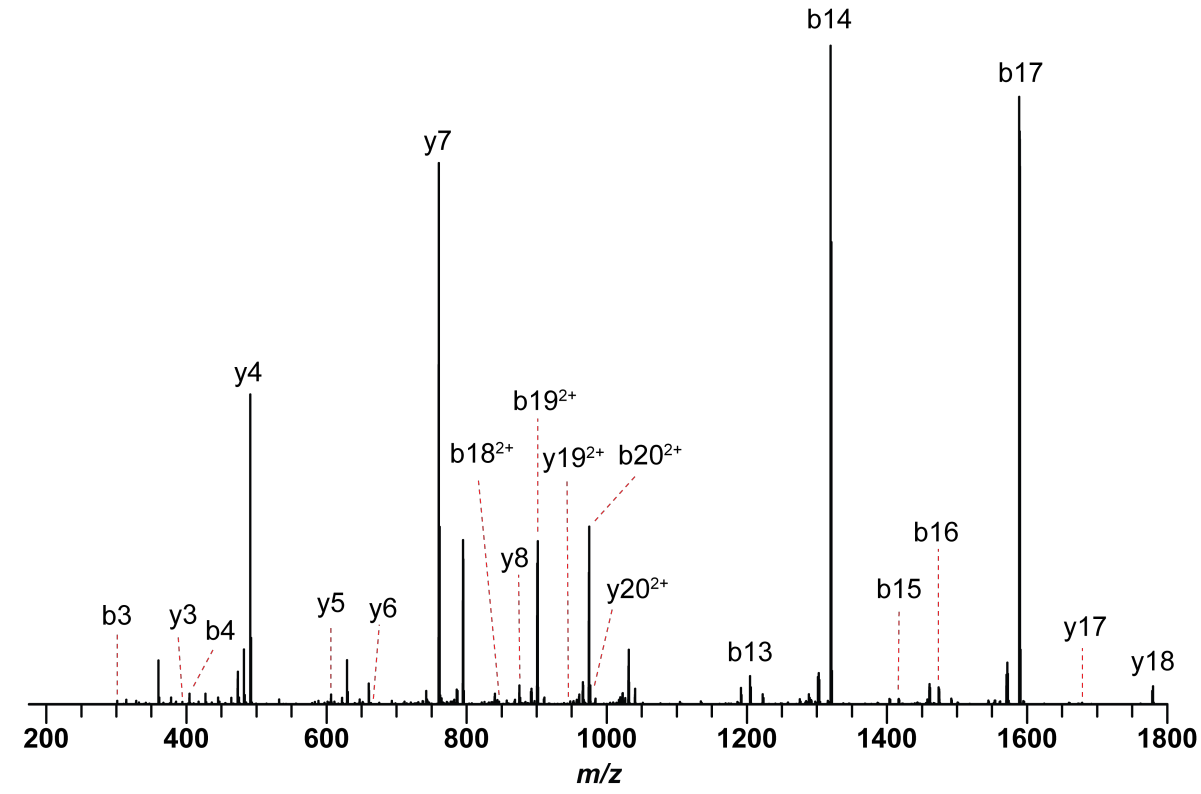

C

\begin{tabular}{cccc}
\hline Ion & Calc. Mass & Obs. Mass & Error $(\mathbf{p p m})$ \\
\hline$[\mathbf{M + 2 H}]^{2+}$ & 1039.9561 & 1039.9558 & 0.3 \\
\hline b3 & 301.1507 & 301.1503 & 1.3 \\
\hline b4 & 402.1983 & 402.1980 & 0.9 \\
b13 & 1204.5341 & 1204.5319 & 1.8 \\
\hline b14 & 1319.5611 & 1319.5584 & 2.0 \\
\hline b15 & 1416.6139 & 1416.6117 & 1.5 \\
\hline b16 & 1473.6353 & 1473.6330 & 1.6 \\
\hline b17 & 1588.6623 & 1588.6597 & 1.6 \\
b18 $^{2+}$ & 843.3611 & 843.3604 & 0.9 \\
\hline b19 & 900.8746 & 900.8732 & 1.6 \\
\hline b20 $^{2+}$ & 974.4088 & 974.4073 & 1.5 \\
\hline
\end{tabular}

\begin{tabular}{|c|c|c|c|}
\hline Ion & Calc. Mass & Obs. Mass & Error (ppm) \\
\hline y3 & 394.1973 & 394.1969 & 1.0 \\
\hline y4 & 491.2500 & 491.2495 & 1.1 \\
\hline y5 & 606.2770 & 606.2762 & 1.3 \\
\hline y6 & 663.2984 & 663.2978 & 0.9 \\
\hline y7 & 760.3512 & 760.3500 & 1.6 \\
\hline y8 & 875.3781 & 875.3770 & 1.3 \\
\hline y17 & 1677.7140 & 1677.7092 & 2.8 \\
\hline y18 & 1778.7616 & 1778.7579 & 2.1 \\
\hline$y 19^{2+}$ & 947.3979 & 947.3964 & 1.6 \\
\hline y20 $2+$ & 975.9087 & 975.9071 & 1.6 \\
\hline
\end{tabular}


D) HRMS/MS of methanolized singly modified product-2 formed by ThtA-T8A variant. A. Fragmentation of methanolized singly modified product-2 formed upon co-expression of ThtAT8A variant with ThtB. Observed $b$ - and $y$-ions are annotated. Green denotes the substituted position. Purple denotes an Asp methyl ester. B. Annotated CID spectrum of fully methanolized singly modified product-2. C. Table of daughter ion assignments.

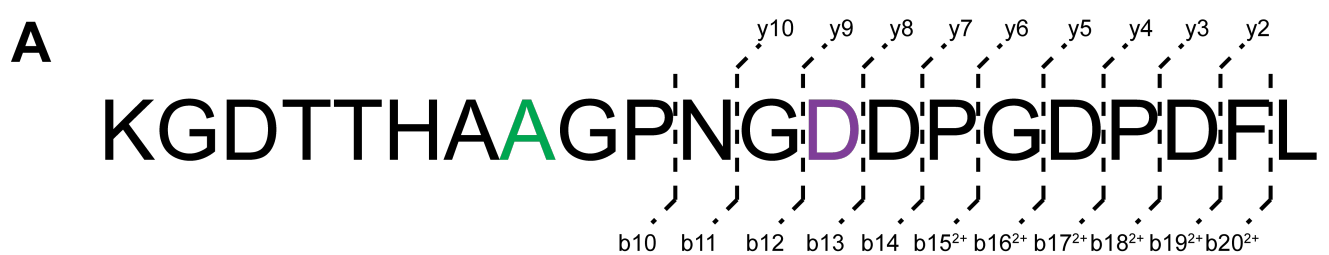

B

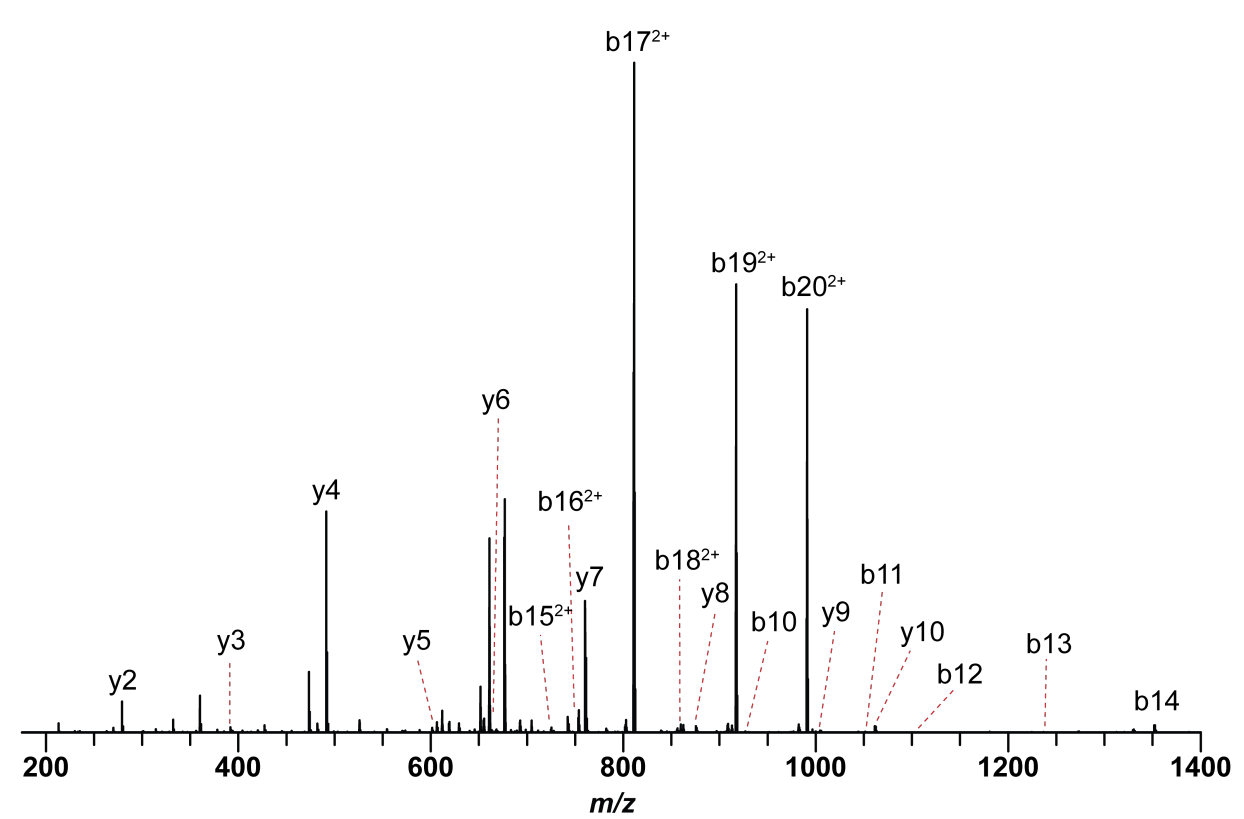

\begin{tabular}{|c|c|c|c|}
\hline Ion & Calc. Mass & Obs. Mass & Error (ppm) \\
\hline$[\mathrm{M}+3 \mathrm{H}]^{3+}$ & 704.3153 & 704.3147 & 0.8 \\
\hline b10 & 936.4534 & 936.4518 & 1.7 \\
\hline b11 & 1050.4963 & 1050.4947 & 1.5 \\
\hline b12 & 1107.5178 & 1107.5157 & 1.9 \\
\hline b13 & 1236.5604 & 1236.5582 & 1.8 \\
\hline b14 & 1351.5873 & 1351.5855 & 1.3 \\
\hline b1 $5^{2+}$ & 724.8237 & 724.8232 & 0.7 \\
\hline b16 ${ }^{2+}$ & 753.3344 & 753.3333 & 1.5 \\
\hline b1 $7^{2+}$ & 810.8479 & 810.8468 & 1.4 \\
\hline b1 $8^{2+}$ & 859.3743 & 859.3731 & 1.4 \\
\hline b1 $9^{2+}$ & 916.8877 & 916.8867 & 1.1 \\
\hline
\end{tabular}

\begin{tabular}{cccc}
\hline Ion & Calc. Mass & Obs. Mass & Error $(\mathbf{p p m})$ \\
\hline b20 $^{2+}$ & 990.4219 & 990.4206 & 1.4 \\
\hline y2 & 279.1703 & 279.1699 & 1.6 \\
y3 & 394.1973 & 394.1968 & 1.2 \\
y4 & 491.2500 & 491.2494 & 1.3 \\
y5 & 606.2770 & 606.2762 & 1.3 \\
y6 & 663.2984 & 663.2976 & 1.2 \\
y7 & 760.3512 & 760.3501 & 1.5 \\
y8 & 875.3781 & 875.3771 & 1.2 \\
y9 & 1004.4207 & 1004.4194 & 1.3 \\
y10 & 1061.4422 & 1061.4406 & 1.6 \\
\hline
\end{tabular}


Figure S28. ThtA-D13A variant-modified products: HRMS/MS.

A) HRMS/MS of singly modified product-1 formed by ThtA-D13A variant. A. Fragmentation of singly modified product-1 formed upon co-expression of ThtA-D13A variant with ThtB. Observed b- and y-ions are annotated. Green denotes the substituted position. The black bracket connecting Thr and Asp indicates the site of macrolactone formation. B. Annotated CID spectrum of singly modified product-1. C. Table of daughter ion assignments.

A

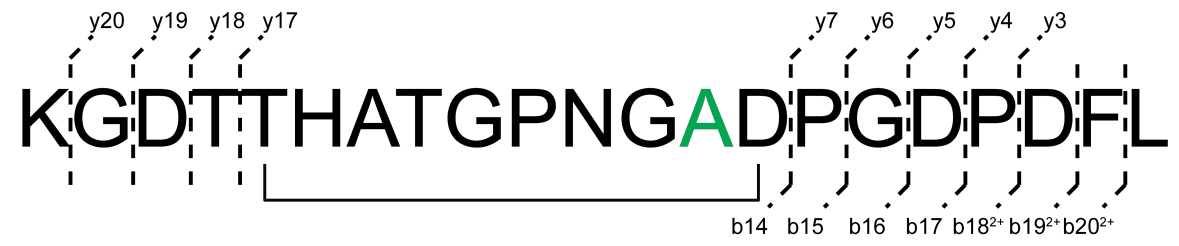

B

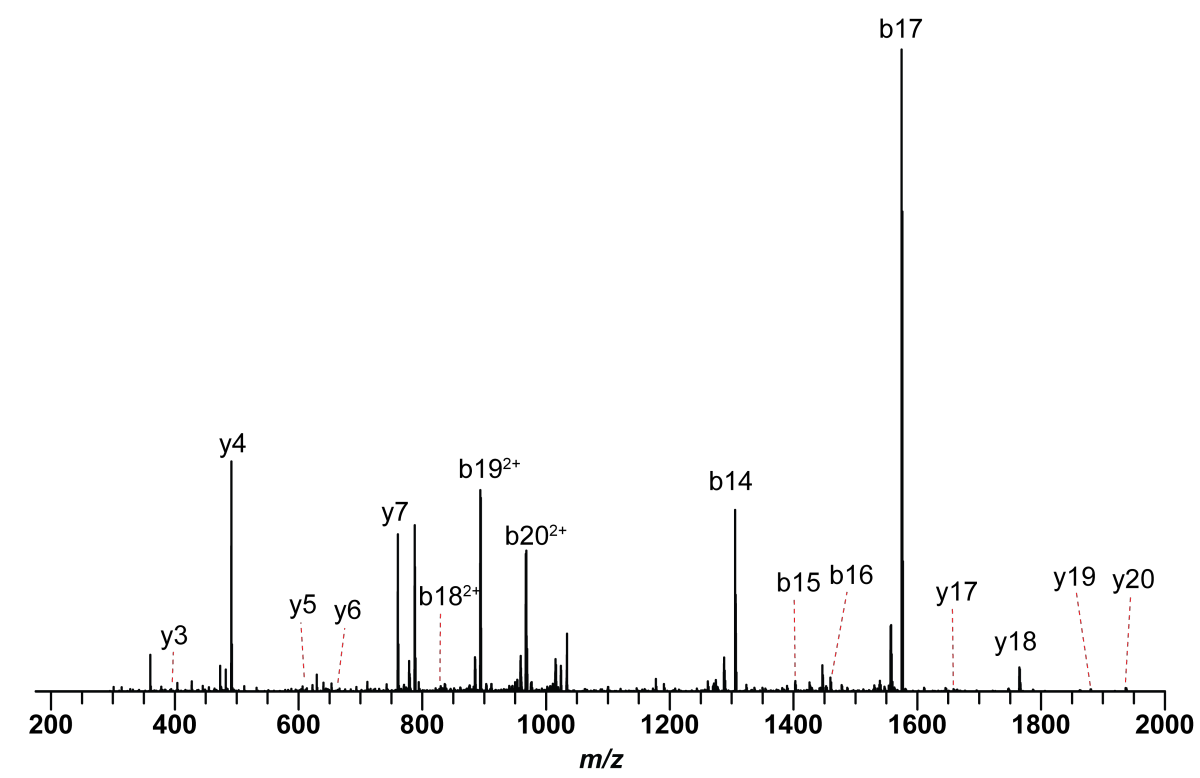

C

\begin{tabular}{cccc}
\hline Ion & Calc. Mass & Obs. Mass & Error (ppm) \\
\hline$[\mathbf{M + 2 H}]^{2+}$ & 1032.9665 & 1032.9660 & 0.5 \\
\hline b14 & 1305.5818 & 1305.5800 & 1.4 \\
b15 & 1402.6346 & 1402.6345 & 0.1 \\
b16 & 1459.6560 & 1459.6535 & 1.7 \\
\hline b17 & 1574.6830 & 1574.6811 & 1.2 \\
b18 $^{2+}$ & 836.3715 & 836.3701 & 1.6 \\
b19 $^{2+}$ & 893.8850 & 893.8833 & 1.9 \\
b20 $^{2+}$ & 967.4192 & 967.4172 & 2.1 \\
\hline y3 & 394.1973 & 394.1962 & 2.7 \\
\hline y & 491.2500 & 491.2492 & 1.7 \\
\hline
\end{tabular}

\begin{tabular}{cccc}
\hline Ion & Calc. Mass & Obs. Mass & Error (ppm) \\
\hline y5 & 606.2770 & 606.2764 & 0.9 \\
y6 & 663.2984 & 663.2978 & 0.9 \\
y7 & 760.3512 & 760.3487 & 3.3 \\
\hline y17 & 1663.7347 & 1663.7312 & 2.1 \\
y18 & 1764.7824 & 1764.7799 & 1.4 \\
y19 & 1879.8093 & 1879.8076 & 0.9 \\
y20 & 1936.8308 & 1936.8249 & 3.0 \\
\hline
\end{tabular}


B) HRMS/MS of methanolized singly modified product-1 formed by ThtA-D13A variant. A. Fragmentation of methanolized singly modified product-1 formed upon co-expression of ThtAD13A variant with ThtB. Observed b- and y-ions are annotated. Green denotes the substituted position. Purple denotes an Asp methyl ester. B. Annotated CID spectrum of fully methanolized singly modified product-1. C. Table of daughter ion assignments.

A

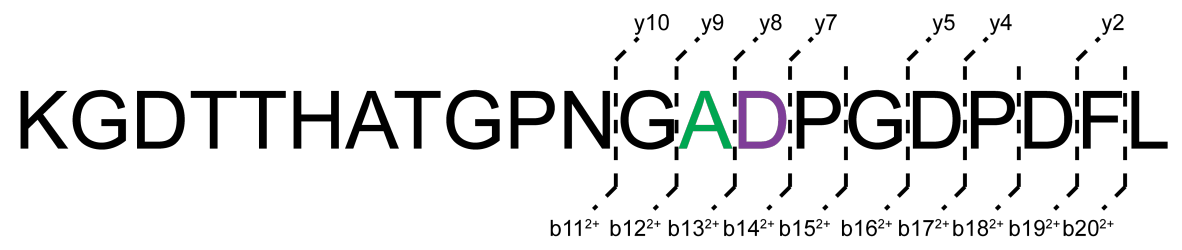

B

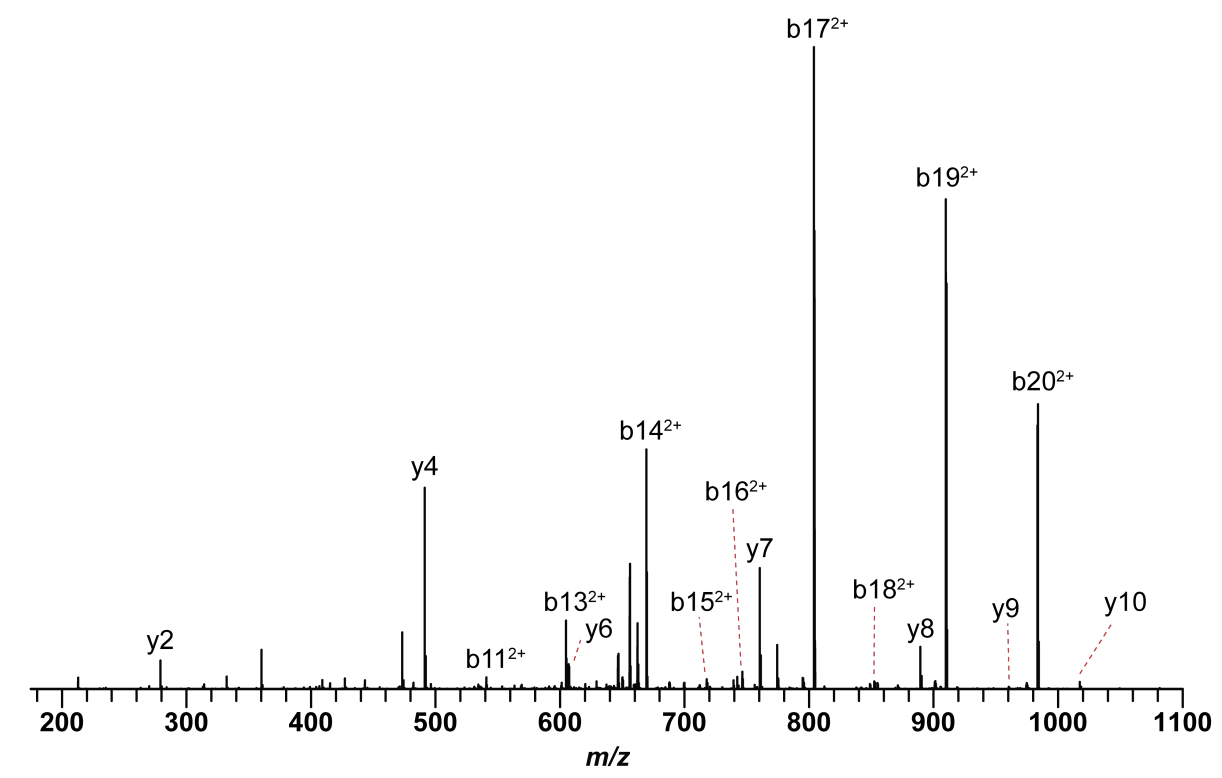

C

\begin{tabular}{cccc}
\hline lon & Calc. Mass & Obs. Mass & Error (ppm) \\
\hline${\text { [M+3H }]^{3+}}^{3} 699.6556$ & 699.6553 & 0.4 \\
b11 $^{2+}$ & 540.7571 & 540.7563 & 1.4 \\
b13 $^{2+}$ & 604.7864 & 604.7858 & 1.0 \\
b14 $^{2+}$ & 669.3077 & 669.3071 & 0.8 \\
\hline b15 $^{2+}$ & 717.8340 & 717.8324 & 2.2 \\
b16 $^{2+}$ & 746.3448 & 746.3433 & 1.9 \\
b17 $^{2+}$ & 803.8582 & 803.8558 & 3.1 \\
b18 $^{2+}$ & 852.3846 & 852.3828 & 2.1 \\
b19 $^{2+}$ & 909.8981 & 909.8965 & 1.8 \\
\hline b20 $^{2+}$ & 983.4323 & 983.4306 & 1.7 \\
\hline
\end{tabular}

\begin{tabular}{cccc}
\hline Ion & Calc. Mass & Obs. Mass & Error $(\mathbf{p p m})$ \\
\hline y2 & 279.1703 & 279.1692 & 3.9 \\
y4 & 491.2500 & 491.2492 & 1.7 \\
y5 & 606.2770 & 606.2763 & 1.2 \\
y7 & 760.3512 & 760.3486 & 3.4 \\
y8 & 889.3938 & 889.3922 & 1.8 \\
y9 & 960.4309 & 960.4289 & 2.0 \\
y10 & 1017.4524 & 1017.4505 & 1.8 \\
\hline
\end{tabular}


C) HRMS/MS of singly modified product-2 formed by ThtA-D13A variant. A. Fragmentation of singly modified product-2 formed upon co-expression of ThtA-D13A variant with ThtB. Observed $b$ - and y-ions are annotated. Green denotes the substituted position. The black bracket connecting Thr and Asp indicates the site of macrolactone formation. B. Annotated CID spectrum of singly modified product-2. C. Table of daughter ion assignments.

A

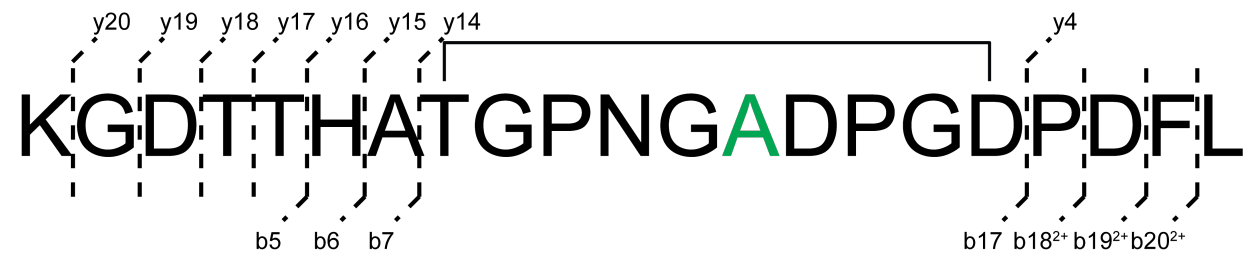

B

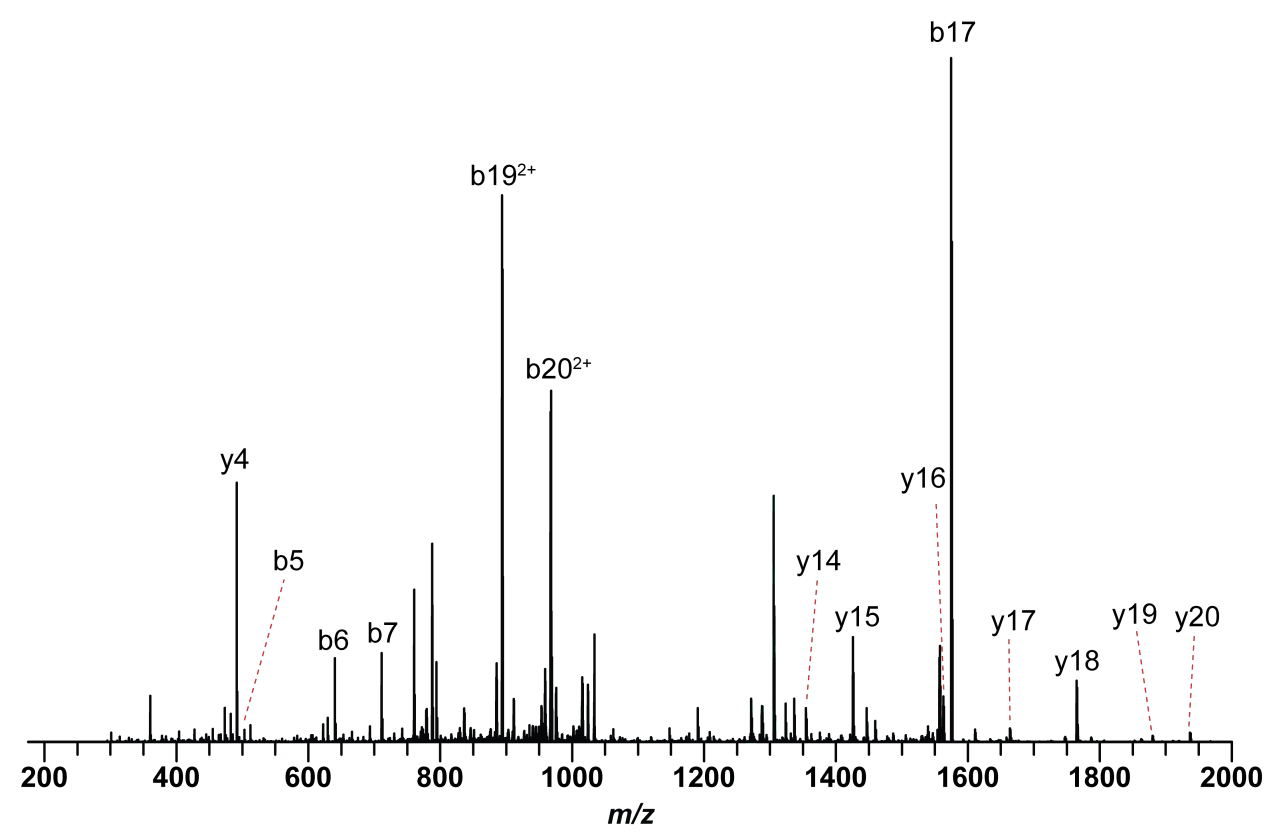

C

\begin{tabular}{cccc}
\hline Ion & Calc. Mass & Obs. Mass & Error $(\mathbf{p p m})$ \\
\hline${\text { [M+2H }]^{2+}}^{\mathbf{M}}$ & 1032.9665 & 1032.9659 & 0.6 \\
b5 & 503.2460 & 503.2451 & 1.8 \\
b6 & 640.3049 & 640.3041 & 1.2 \\
b7 & 711.3420 & 711.3402 & 2.5 \\
b17 & 1574.6830 & 1574.6818 & 0.8 \\
b18 $^{2+}$ & 836.3715 & 836.3698 & 2.0 \\
b19 $^{2+}$ & 893.8850 & 893.8833 & 1.9 \\
b20 $^{2+}$ & 967.4192 & 967.4174 & 1.8 \\
$\mathbf{y 4}$ & 491.2500 & 491.2492 & 1.6 \\
$\mathbf{y 1 4}$ & 1354.5910 & 1354.5896 & 1.0 \\
\hline
\end{tabular}

\begin{tabular}{cccc}
\hline Ion & Calc. Mass & Obs. Mass & Error (ppm) \\
\hline y15 & 1425.6281 & 1425.6252 & 2.0 \\
y16 & 1562.6870 & 1562.6857 & 0.8 \\
y17 & 1663.7347 & 1663.7318 & 1.7 \\
y18 & 1764.7824 & 1764.7796 & 1.6 \\
y19 & 1879.8093 & 1879.8070 & 1.2 \\
y20 & 1936.8308 & 1936.8249 & 3.0 \\
\hline
\end{tabular}


D) HRMS/MS of methanolized singly modified product-2 by ThtA-D13A variant. A. Fragmentation of methanolized singly modified product-2 formed upon co-expression of ThtAD13A variant with ThtB. Observed b- and y-ions are annotated. Green denotes the substituted position. Purple denotes an Asp methyl ester. B. Annotated CID spectrum of fully methanolized singly modified product-2. C. Table of daughter ion assignments.

A

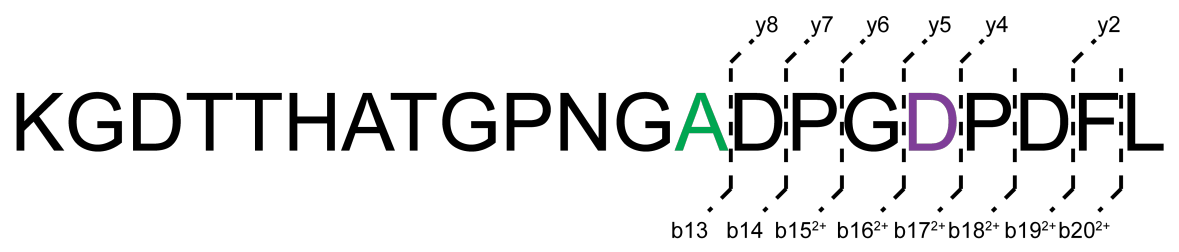

B

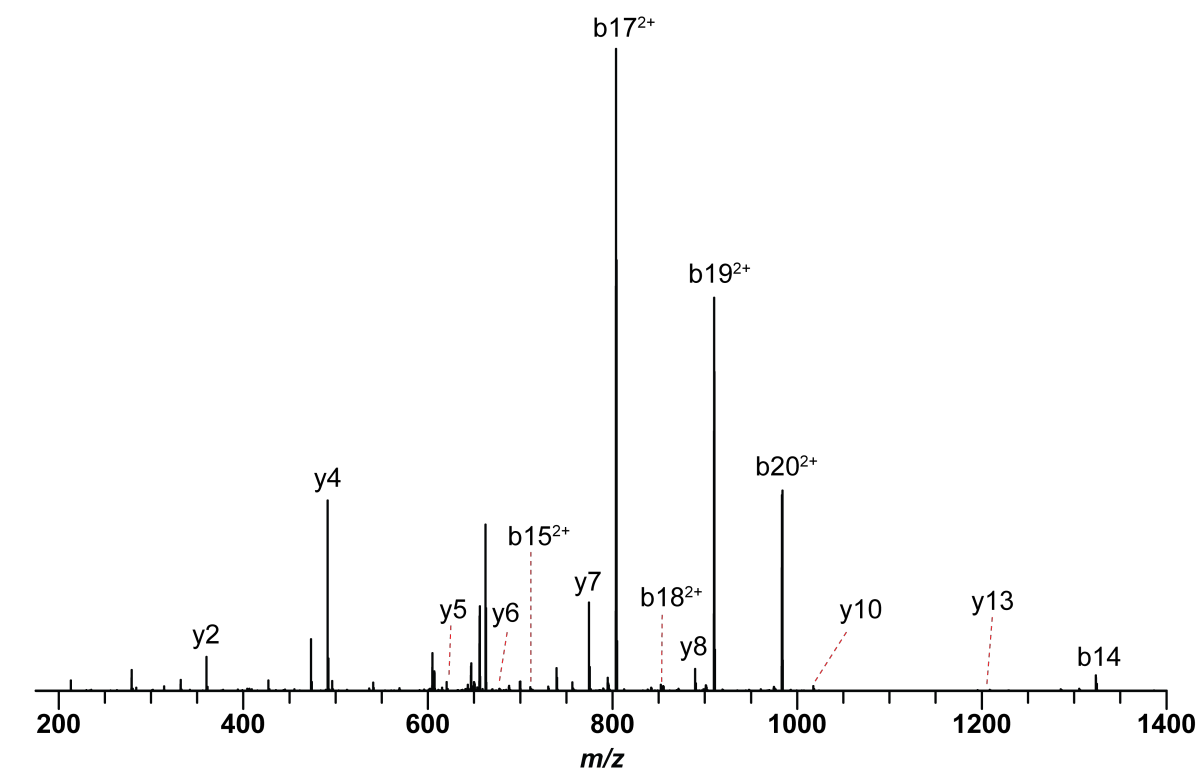

C

\begin{tabular}{cccc}
\hline Ion & Calc. Mass & Obs. Mass & Error (ppm) \\
\hline$\left[\begin{array}{c}\text { M+3H }]^{3+} \\
\text { b13 }\end{array}\right.$ & 699.6556 & 699.6554 & 0.2 \\
b14 & 1208.5654 & 1208.5640 & 1.2 \\
b15 $^{2+}$ & 710.8262 & 710.8245 & 2.4 \\
b17 $^{2+}$ & 803.8582 & 803.8559 & 3.0 \\
b18 $^{2+}$ & 852.3846 & 852.3827 & 2.3 \\
b19 $^{2+}$ & 909.8981 & 909.8965 & 1.7 \\
\hline b20 & & & \\
y2 & 983.4323 & 983.4304 & 2.0 \\
y4 & 279.1703 & 279.1693 & 3.8 \\
\hline & 491.2500 & 491.2491 & 1.9 \\
\hline
\end{tabular}

\begin{tabular}{cccc}
\hline Ion & Calc. Mass & Obs. Mass & Error (ppm) \\
\hline y5 & 620.2926 & 620.2918 & 1.3 \\
y6 & 677.3141 & 677.3123 & 2.7 \\
y7 & 774.3669 & 774.3642 & 3.4 \\
y8 & 889.3938 & 889.3923 & 1.7 \\
\hline
\end{tabular}


Figure S29. ThtA-D17A variant-modified products: HRMS/MS.

A) HRMS/MS of singly modified product-1 formed by ThtA-D17A variant. A. Fragmentation of singly modified product-1 formed upon co-expression of ThtA-D17A variant with ThtB. Observed b- and y-ions are annotated. Green denotes the substituted position. The black bracket connecting Thr and Asp indicates the site of macrolactone formation. B. Annotated CID spectrum of singly modified product-1. C. Table of daughter ion assignments.

A

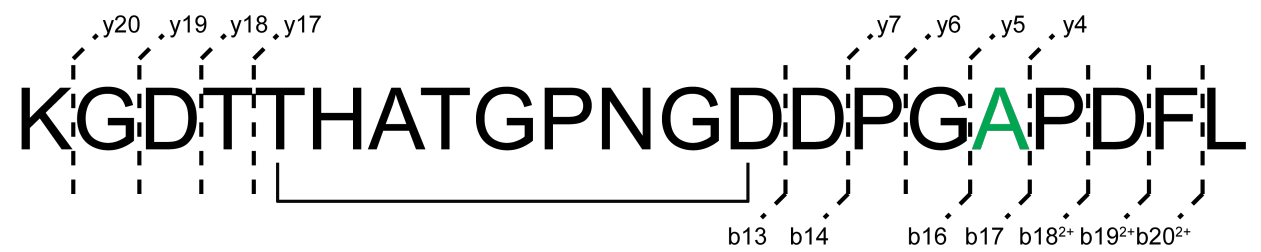

B

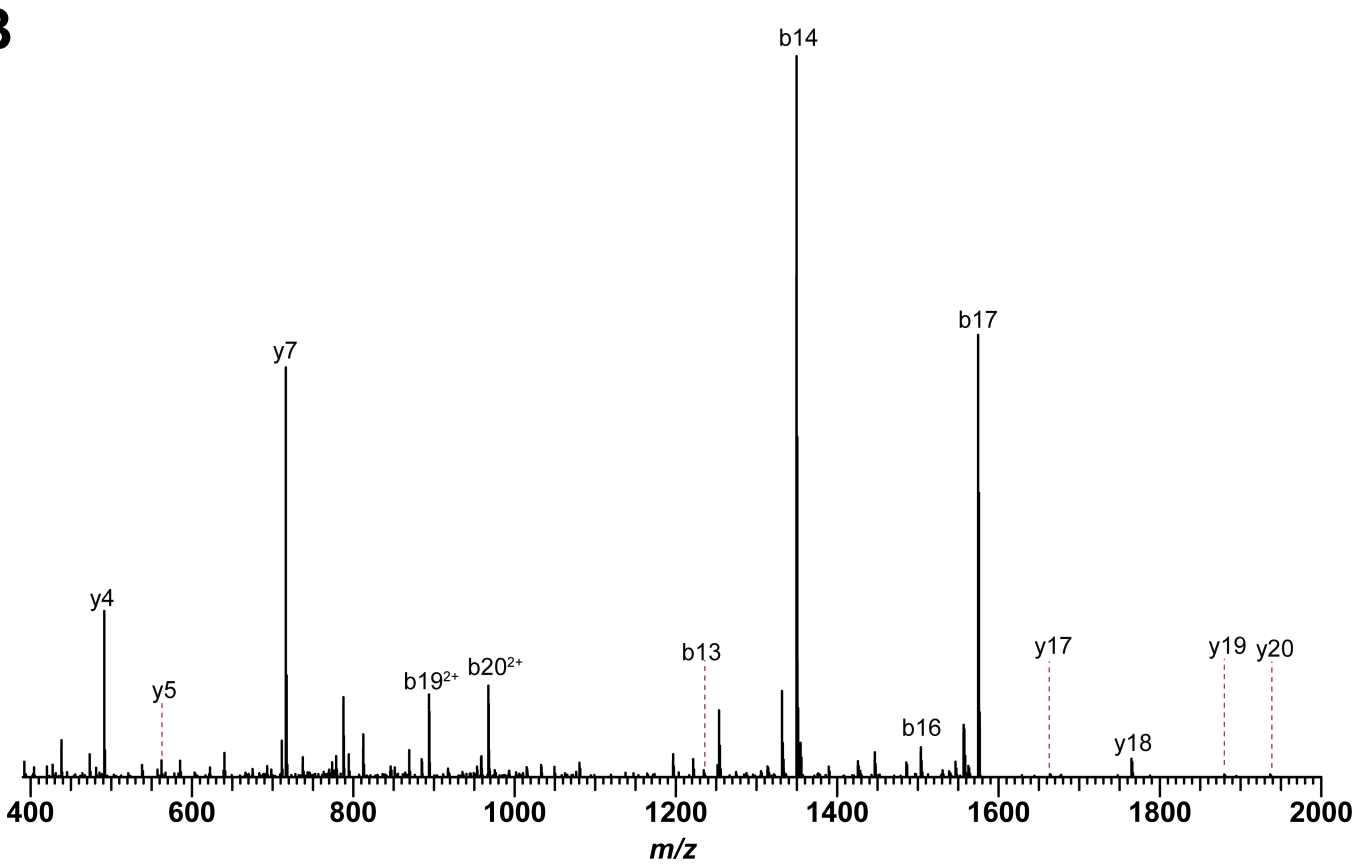

C

\begin{tabular}{cccc}
\hline Ion & Calc. Mass & Obs. Mass & $\begin{array}{c}\text { Error } \\
\text { (in ppm) }\end{array}$ \\
\hline$[\mathbf{M + 2 H}]^{2+}$ & 1032.9665 & 1032.9658 & 0.7 \\
b13 & 1234.5447 & 1234.5428 & 1.5 \\
b14 & 1349.5717 & 1349.5702 & 1.1 \\
b16 & 1503.6459 & 1503.6442 & 1.1 \\
b17 & 1574.6830 & 1574.6818 & 0.8 \\
b18 $^{2+}$ & 836.3715 & 836.3697 & 2.2 \\
b19 $^{2+}$ & 893.8850 & 893.8834 & 1.8 \\
\hline b20 $^{2+}$ & 967.4192 & 967.4174 & 1.9 \\
\hline
\end{tabular}

\begin{tabular}{cccc}
\hline Ion & Calc. Mass & Obs. Mass & $\begin{array}{c}\text { Error } \\
\text { (in ppm) }\end{array}$ \\
\hline y4 & 491.2500 & 491.2492 & 1.6 \\
y5 & 562.2871 & 562.2862 & 1.6 \\
y6 & 619.3086 & 619.3077 & 1.5 \\
y7 & 716.3614 & 716.3596 & 2.5 \\
y17 & 1663.7347 & 1663.7323 & 1.5 \\
y18 & 1764.7824 & 1764.7796 & 1.6 \\
y19 & 1879.8093 & 1879.8065 & 1.5 \\
\hline y20 & 1936.8308 & 1936.8256 & 2.7 \\
\hline
\end{tabular}


B) HRMS/MS of methanolized singly modified product-1 formed by ThtA-D17A variant. A. Fragmentation of methanolized singly modified product-1 formed upon co-expression of ThtAD17A variant with ThtB. Observed b- and y-ions are annotated. Green denotes the substituted position. Purple denotes an Asp methyl ester. B. Annotated CID spectrum of fully methanolized singly modified product-1. C. Table of daughter ion assignments.

A

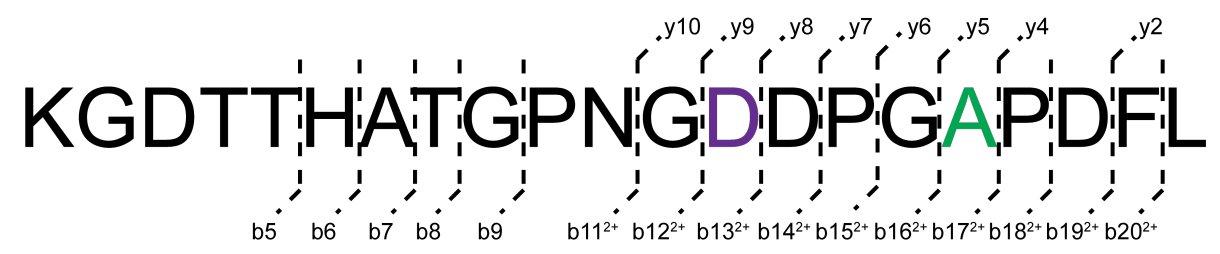

B

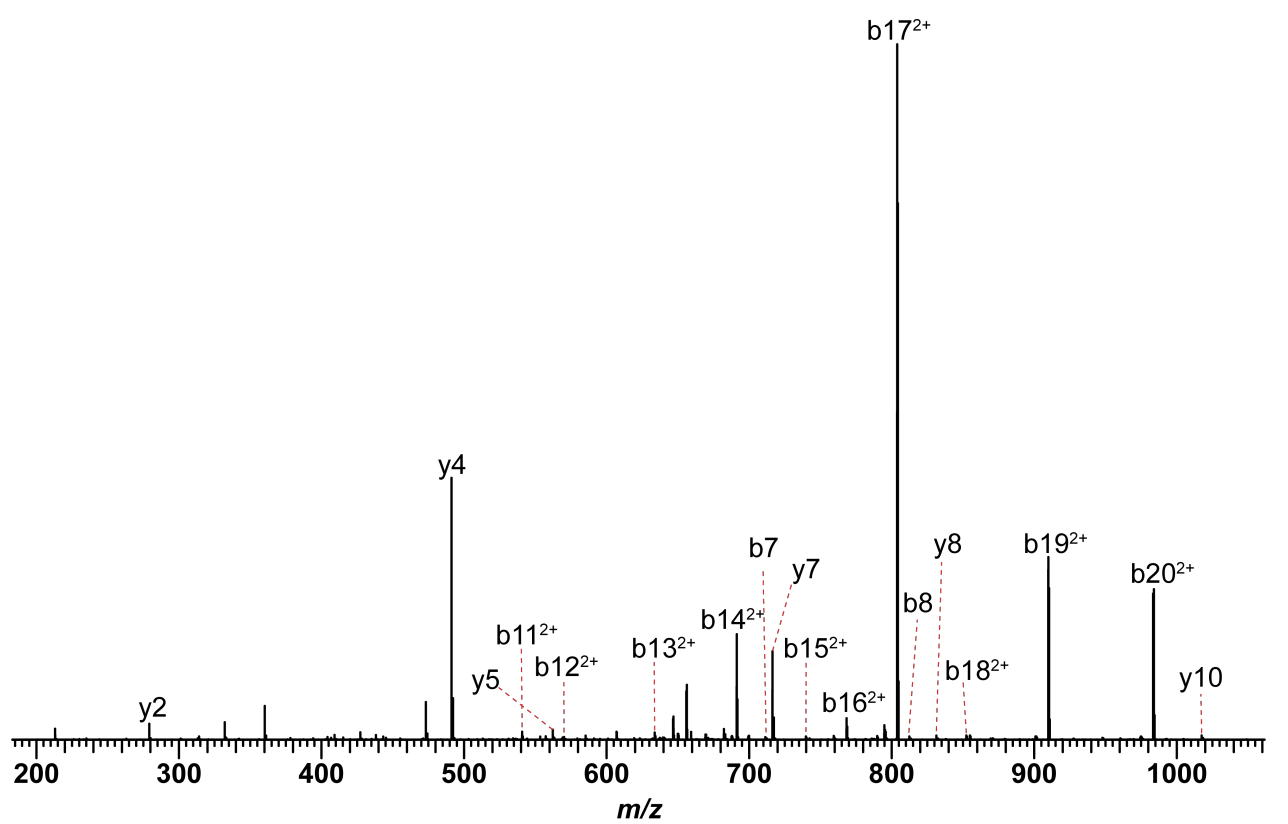

C

\begin{tabular}{cccc}
\hline Ion & Calc. Mass & Obs. Mass & $\begin{array}{c}\text { Error } \\
\text { (in ppm) }\end{array}$ \\
\hline${\text { [M+3H }]^{3+}}^{3+}$ & 699.6556 & 699.6552 & 0.6 \\
\hline b5 & 503.2460 & 503.2451 & 1.7 \\
\hline b6 & 640.3049 & 640.3040 & 1.4 \\
\hline b7 & 711.3420 & 711.3404 & 2.3 \\
\hline b8 & 812.3897 & 812.3869 & 3.5 \\
\hline b9 & 869.4112 & 869.4092 & 2.3 \\
\hline b112+ & 540.7571 & 540.7565 & 1.1 \\
\hline b12 $^{2+}$ & 569.2678 & 569.2671 & 1.2 \\
\hline b13 $^{2+}$ & 633.7891 & 633.7883 & 1.2 \\
\hline b14 $^{2+}$ & 691.3026 & 691.3010 & 2.3 \\
\hline b15 $^{2+}$ & 739.8290 & 739.8274 & 2.1 \\
\hline b16 $^{2+}$ & 768.3397 & 768.3371 & 3.4 \\
\hline
\end{tabular}

\begin{tabular}{cccc}
\hline Ion & Calc. Mass & Obs. Mass & $\begin{array}{c}\text { Error } \\
\text { (in ppm) }\end{array}$ \\
\hline b17 $^{2+}$ & 803.8582 & 803.8559 & 2.9 \\
\hline b18 $^{2+}$ & 852.3846 & 852.3827 & 2.3 \\
\hline b19 $^{2+}$ & 909.8981 & 909.8964 & 1.9 \\
b20 $^{2+}$ & 983.4323 & 983.4302 & 2.1 \\
\hline y2 & 279.1703 & 279.1692 & 3.9 \\
\hline y4 & 491.2500 & 491.2491 & 1.9 \\
\hline y5 & 562.2871 & 562.2864 & 1.4 \\
\hline y6 & 619.3086 & 619.3078 & 1.3 \\
\hline $\mathbf{y 7}$ & 716.3614 & 716.3598 & 2.2 \\
\hline y8 & 831.3883 & 831.3858 & 3.0 \\
\hline y9 & 960.4309 & 960.4286 & 2.4 \\
\hline y10 & 1017.4524 & 1017.4504 & 2.0 \\
\hline
\end{tabular}


C) HRMS/MS of singly modified product-2 formed by ThtA-D17A variant. A. Fragmentation of singly modified product-2 formed upon co-expression of ThtA-D17A variant with ThtB. Observed $b$ - and y-ions are annotated. Green denotes the substituted position. The black bracket connecting Thr and Asp indicates the site of macrolactone formation. B. Annotated CID spectrum of singly modified product-2. C. Table of daughter ion assignments.

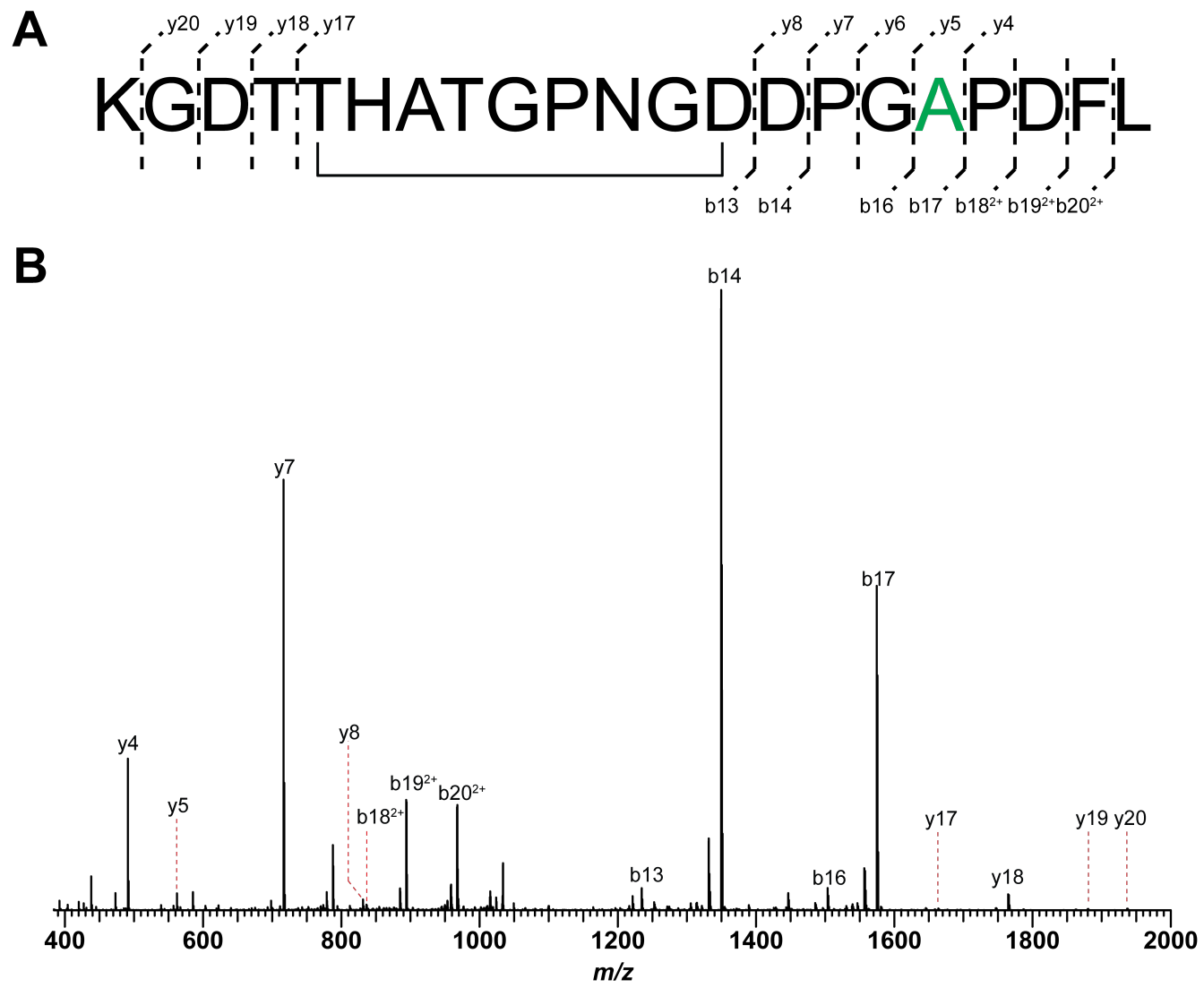

\begin{tabular}{cccc}
\hline $\mathbf{C}$ lon & Calc. Mass & Obs. Mass & $\begin{array}{c}\text { Error } \\
\text { (in ppm) }\end{array}$ \\
\hline$[\mathbf{M + 2 H}]^{2+}$ & 1032.9665 & 1032.9659 & 0.6 \\
\hline $\mathbf{b 1 3}$ & 1234.5447 & 1234.5426 & 1.7 \\
$\mathbf{b 1 4}$ & 1349.5717 & 1349.5703 & 1.0 \\
$\mathbf{b 1 6}$ & 1503.6459 & 1503.6444 & 1.0 \\
$\mathbf{b 1 7}$ & 1574.6830 & 1574.6819 & 0.7 \\
b18 $^{2+}$ & 836.3715 & 836.3699 & 1.9 \\
b19 $^{2+}$ & 893.8850 & 893.8834 & 1.8 \\
b20 $^{2+}$ & 967.4192 & 967.4175 & 1.7 \\
\hline
\end{tabular}

\begin{tabular}{cccc}
\hline Ion & Calc. Mass & Obs. Mass & $\begin{array}{c}\text { Error } \\
\text { (in ppm) }\end{array}$ \\
\hline y4 & 491.2500 & 491.2493 & 1.6 \\
\hline y5 & 562.2871 & 562.2863 & 1.6 \\
y6 & 619.3086 & 619.3078 & 1.2 \\
y7 & 716.3614 & 716.3597 & 2.4 \\
y8 & 831.3883 & 831.3861 & 2.6 \\
\hline 17 & 1663.7347 & 1663.7323 & 1.4 \\
y18 & 1764.7824 & 1764.7797 & 1.5 \\
\hline y19 & 1879.8093 & 1879.8062 & 1.7 \\
\hline y20 & 1936.8308 & 1936.8248 & 3.1 \\
\hline
\end{tabular}


D) HRMS/MS of methanolized singly modified product-2 formed by ThtA-D17A variant. A. Fragmentation of methanolized singly modified product-2 formed upon co-expression of ThtAD17A variant with ThtB. Observed b- and y-ions are annotated. Green denotes the substituted position. Purple denotes an Asp methyl ester. B. Annotated CID spectrum of fully methanolized singly modified product-2. C. Table of daughter ion assignments.

A

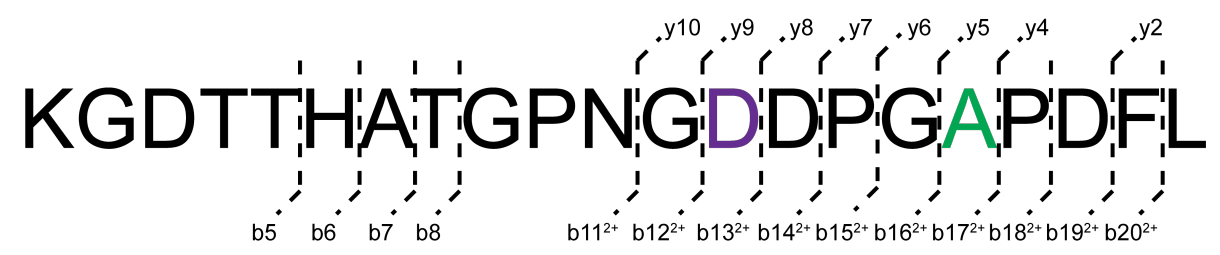

B

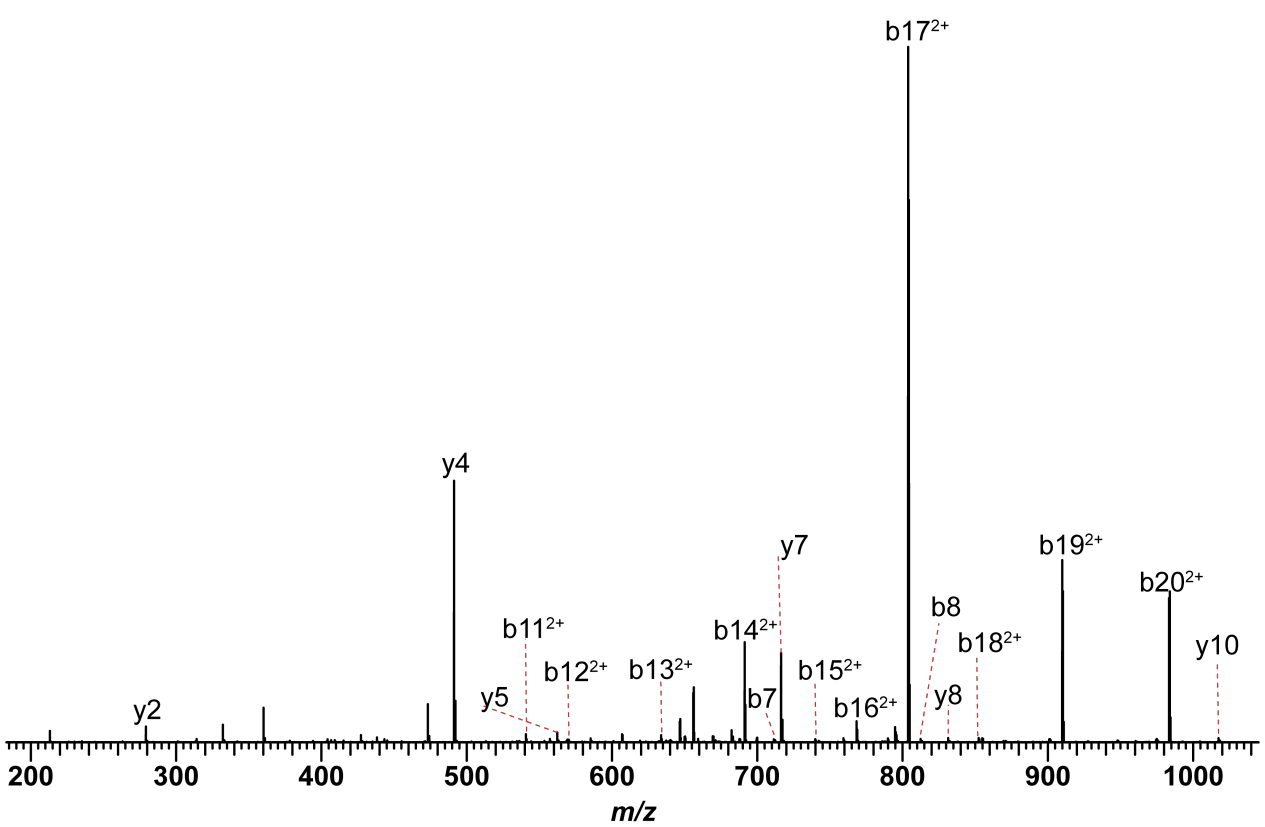

C

\begin{tabular}{|cccc}
\hline Ion & Calc. Mass & Obs. Mass & Error (in ppm) \\
\hline${\text { [M+3H }]^{3+}}^{3}$ & 699.6556 & 699.6553 & 0.4 \\
\hline b5 & 503.2460 & 503.2451 & 1.7 \\
\hline b6 & 640.3049 & 640.3040 & 1.4 \\
\hline b7 & 711.3420 & 711.3404 & 2.3 \\
\hline b8 & 812.3897 & 812.3869 & 3.4 \\
b11 $^{2+}$ & 540.7571 & 540.7565 & 1.0 \\
\hline b12 $^{2+}$ & 569.2678 & 569.2671 & 1.2 \\
\hline b13 $^{2+}$ & 633.7891 & 633.7883 & 1.3 \\
\hline b14 $^{2+}$ & 691.3026 & 691.3010 & 2.3 \\
\hline b15 $^{2+}$ & 739.8290 & 739.8275 & 2.0 \\
\hline b16 $^{2+}$ & 768.3397 & 768.3371 & 3.3 \\
\hline b17 $^{2+}$ & 803.8582 & 803.8560 & 2.8 \\
\hline
\end{tabular}

\begin{tabular}{cccc}
\hline Ion & Calc. Mass & Obs. Mass & Error (in ppm) \\
\hline b18 $^{2+}$ & 852.3846 & 852.3827 & 2.3 \\
b19 $^{2+}$ & 909.8981 & 909.8963 & 1.9 \\
b20 $^{2+}$ & 983.4323 & 983.4302 & 2.2 \\
y2 & 279.1703 & 279.1693 & 3.8 \\
y4 & 491.2500 & 491.2492 & 1.6 \\
y5 & 562.2871 & 562.2864 & 1.4 \\
y6 & 619.3086 & 619.3077 & 1.5 \\
y7 & 716.3614 & 716.3598 & 2.3 \\
y8 & 831.3883 & 831.3861 & 2.6 \\
y9 & 960.4309 & 960.4288 & 2.2 \\
y10 & 1017.4524 & 1017.4504 & 2.0 \\
\hline
\end{tabular}


Figure S30. Iso-thatisin: HRMS/MS.

A) HRMS/MS of iso-thatisin. A. Fragmentation of iso-thatisin. Observed b- and y-ions are annotated. Black brackets connecting Thr and Asp indicate sites of macrolactone formation. B. Annotated CID spectrum of iso-thatisin. C. Table of daughter ion assignments.

A

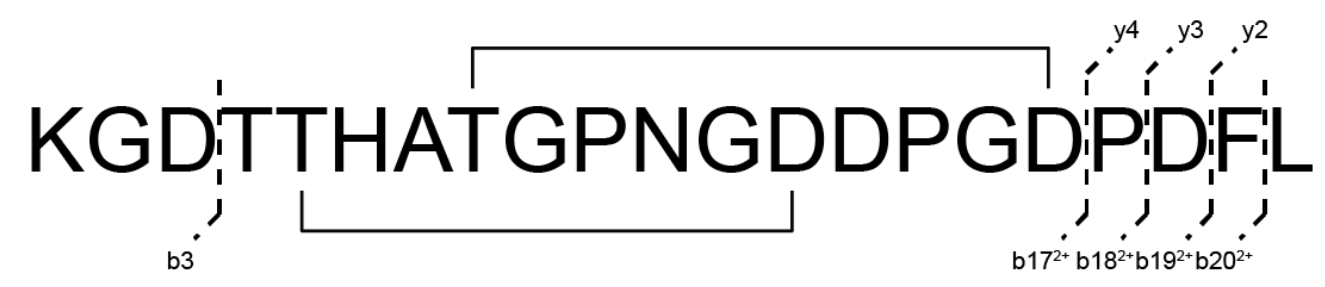

B

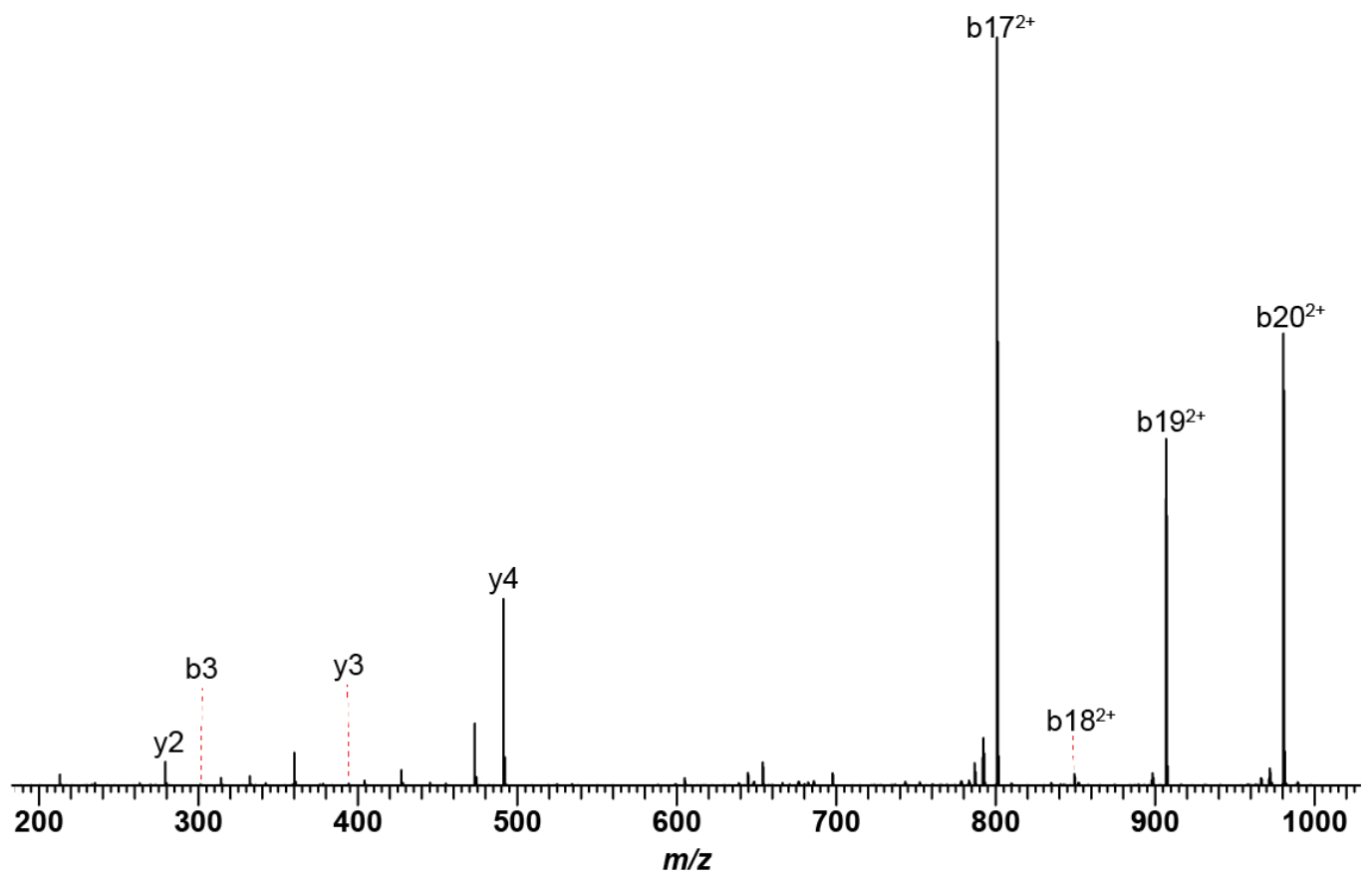

\begin{tabular}{|c|c|c|c|}
\hline Ion & Calc. Mass & Obs. Mass & Error (ppm) \\
\hline$[\mathrm{M}+3 \mathrm{H}]^{3+}$ & 697.6399 & 697.6403 & 0.6 \\
\hline b3 & 301.1507 & 301.1496 & 3.6 \\
\hline b1 $17^{2+}$ & 800.8348 & 800.8323 & 3.1 \\
\hline b18 $8^{2+}$ & 849.3611 & 849.3591 & 2.4 \\
\hline b1 $19^{2+}$ & 906.8746 & 906.8727 & 2.2 \\
\hline b20 $0^{2+}$ & 980.4088 & 980.4068 & 2.1 \\
\hline y2 & 279.1703 & 279.1693 & 3.8 \\
\hline$y^{3}$ & 394.1973 & 394.1961 & 3.0 \\
\hline$y 4$ & 491.2500 & 491.2491 & 1.9 \\
\hline
\end{tabular}


B) HRMS/MS of fully methanolized iso-thatisin. A. Fragmentation of fully methanolized isothatisin. Observed $b$ - and $y$-ions are annotated. Purple indicates an Asp methyl ester. B. Annotated CID spectrum of fully methanolized iso-thatisin. C. Table of daughter ion assignments.

A

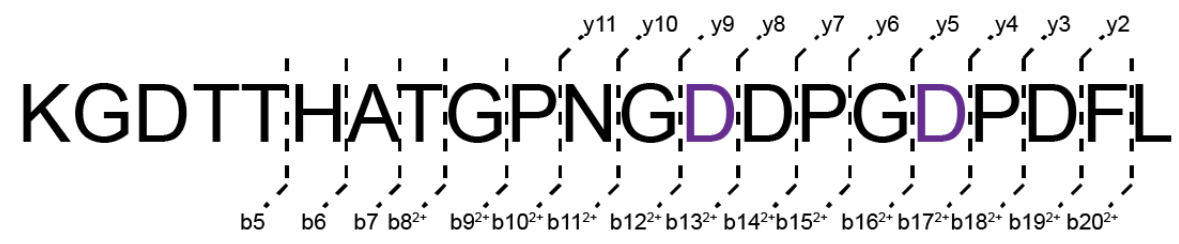

B

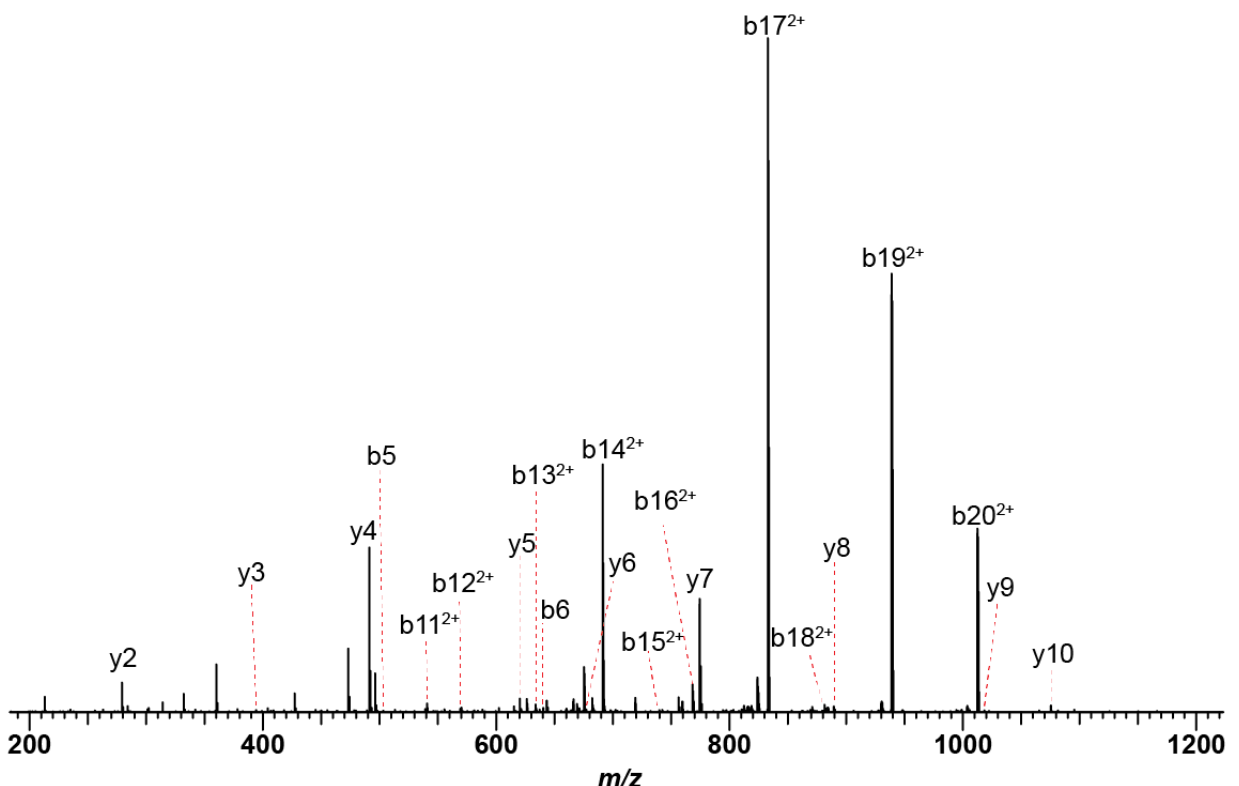

C

\begin{tabular}{cccc}
\hline Ion & Calc. Mass & Obs. Mass & Error (ppm) \\
\hline${\text { [M+3H }]^{3+}}^{3}$ & 718.9907 & 718.9908 & 0.2 \\
\hline b5 & 503.2460 & 503.2445 & 3.0 \\
\hline b6 & 640.3049 & 640.3038 & 1.7 \\
\hline b7 & 711.3420 & 711.3400 & 2.9 \\
\hline b8 $^{2+}$ & 406.6985 & 406.6973 & 2.9 \\
\hline b9 $^{2+}$ & 435.2092 & 435.2081 & 2.5 \\
\hline b10 $^{2+}$ & 483.7356 & 483.7346 & 2.1 \\
\hline b11 $^{2+}$ & 540.7571 & 540.7562 & 1.5 \\
\hline b12 $^{2+}$ & 569.2678 & 569.2668 & 1.7 \\
\hline b13 $^{2+}$ & 633.7891 & 633.7880 & 1.7 \\
\hline b14 & \\
\hline b15 $^{2+}$ & 691.3026 & 691.3006 & 2.8 \\
\hline b16 & 739.8290 & 739.8272 & 2.3 \\
\hline b17 & 768.3397 & 768.3367 & 3.9 \\
\hline & 832.8610 & 832.8585 & 3.0 \\
\hline
\end{tabular}

\begin{tabular}{|c|c|c|c|}
\hline Ion & Calc. Mass & Obs. Mass & Error (ppm) \\
\hline b18 $2^{2+}$ & 881.3874 & 881.3855 & 2.1 \\
\hline b1 $19^{2+}$ & 938.9008 & 938.8990 & 2.0 \\
\hline b202+ & 1012.4350 & 1012.4329 & 2.1 \\
\hline y2 & 279.1703 & 279.1691 & 4.3 \\
\hline y3 & 394.1973 & 394.1961 & 3.0 \\
\hline y4 & 491.2500 & 491.2490 & 2.1 \\
\hline y5 & 620.2926 & 620.2915 & 1.9 \\
\hline y6 & 677.3141 & 677.3117 & 3.5 \\
\hline y7 & 774.3669 & 774.3638 & 3.9 \\
\hline y8 & 889.3938 & 889.3918 & 2.2 \\
\hline y9 & 1018.4364 & 1018.4343 & 2.1 \\
\hline y10 & 1075.4579 & 1075.4552 & 2.5 \\
\hline y11 & 1189.5008 & 1189.4988 & 1.7 \\
\hline
\end{tabular}


C) HRMS/MS of partially methanolized iso-thatisin. A. Fragmentation of partially methanolized iso-thatisin. Observed b- and y-ions are annotated. Purple indicates an Asp methyl ester. The black bracket connecting Thr and Asp indicates the site of macrolactone formation. B. Annotated $\mathrm{CID}$ spectrum of partially methanolized iso-thatisin. C. Table of daughter ion assignments.

A

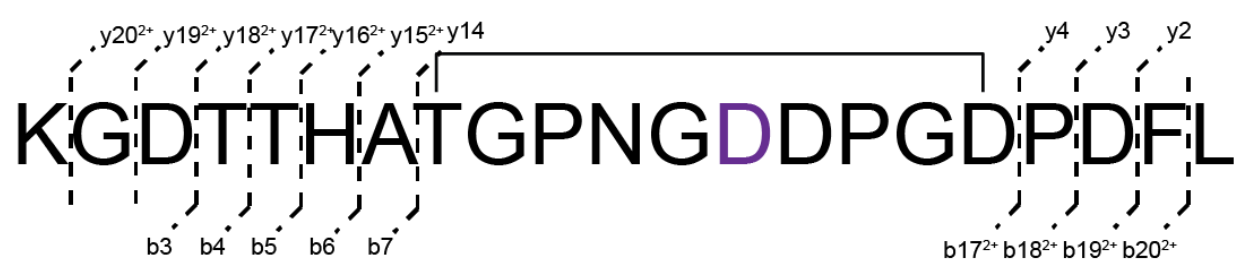

B

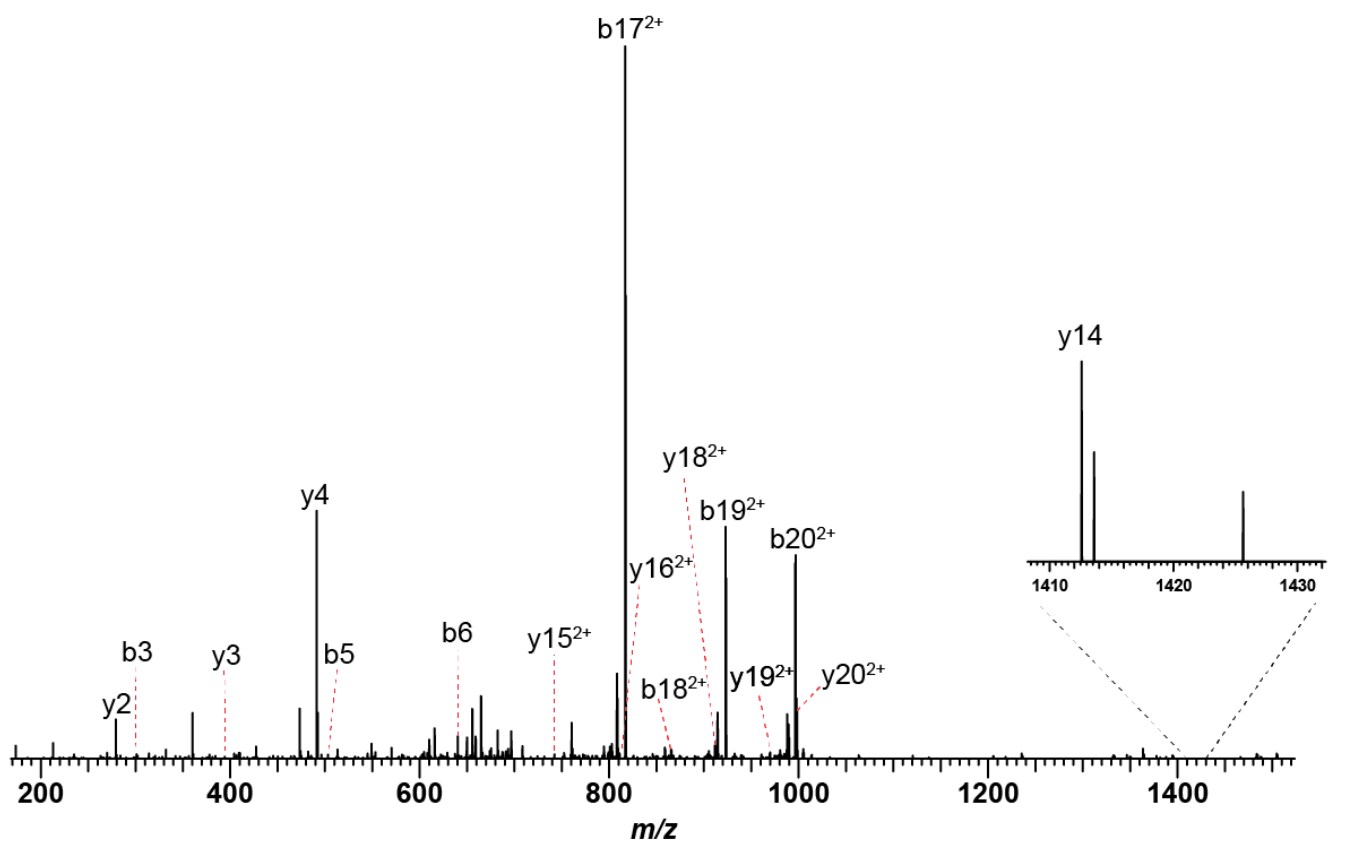

C

\begin{tabular}{|c|c|c|c|}
\hline Ion & Calc. Mass & Obs. Mass & Error (ppm) \\
\hline$[\mathrm{M}+3 \mathrm{H}]^{3+}$ & 708.3153 & 708.3150 & 0.3 \\
\hline b3 & 301.1507 & 301.1496 & 3.6 \\
\hline b4 & 402.1983 & 402.1971 & 2.9 \\
\hline b5 & 503.2460 & 503.2453 & 1.5 \\
\hline b6 & 640.3049 & 640.3042 & 1.1 \\
\hline b7 & 711.3420 & 711.3406 & 2.0 \\
\hline b1 $17^{2+}$ & 816.8479 & 816.8458 & 2.6 \\
\hline b18 ${ }^{2+}$ & 865.3743 & 865.3730 & 1.5 \\
\hline b192+ & 922.8877 & 922.8862 & 1.7 \\
\hline b20 ${ }^{2+}$ & 996.4219 & 996.4200 & 1.9 \\
\hline
\end{tabular}

\begin{tabular}{cccc}
\hline Ion & Calc. Mass & Obs. Mass & Error (ppm) \\
\hline $\mathbf{y 2}$ & 279.1703 & 279.1693 & 3.8 \\
\hline $\mathbf{y 3}$ & 394.1973 & 394.1962 & 2.6 \\
$\mathbf{y 4}$ & 491.2500 & 491.2492 & 1.6 \\
$\mathbf{y 1 4}$ & 1412.5965 & 1412.5932 & 2.3 \\
$\mathbf{y 1 5 ^ { 2 + }}$ & 742.3204 & 742.3187 & 2.3 \\
$\mathbf{y 1 6}^{2+}$ & 810.8499 & 810.8479 & 2.5 \\
$\mathbf{y 1 7}^{2+}$ & 861.3737 & 861.3719 & 2.1 \\
$\mathbf{y 1 8}^{2+}$ & 911.8976 & 911.8960 & 1.8 \\
$\mathbf{y 1 9}^{2+}$ & 969.4110 & 969.4091 & 2.0 \\
$\mathbf{y 2 0}{ }^{2+}$ & 997.9218 & 997.9196 & 2.2 \\
\hline
\end{tabular}


Figure S31. Marfey's analysis of thatisin and iso-thatisin. Ion count-normalized LC-MS chromatograms of FDAA-labeled standards (top) and samples (bottom) are shown. For the standards, the $L$ and $D$ amino acid were run independently and are overlaid. $\mathbf{A}$. Thatisin hydrolysate sample. B. Iso-thatisin hydrolysate sample.

\section{A}
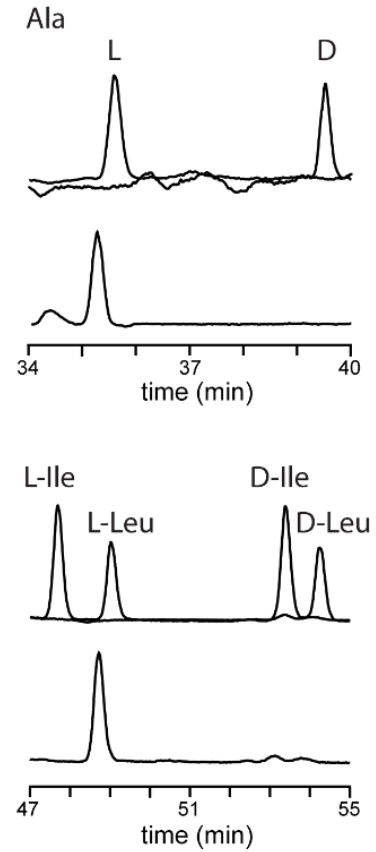

B

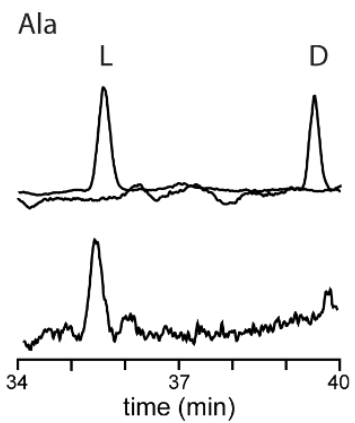

wite thea bite oteen
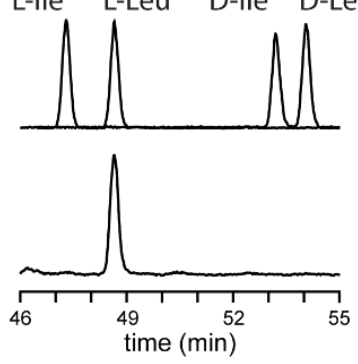

Asp
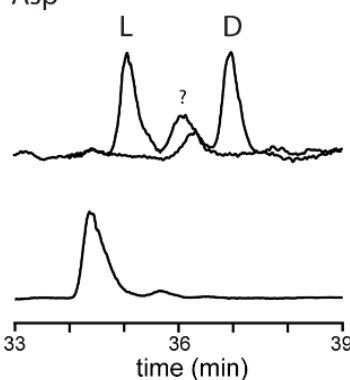

Lys
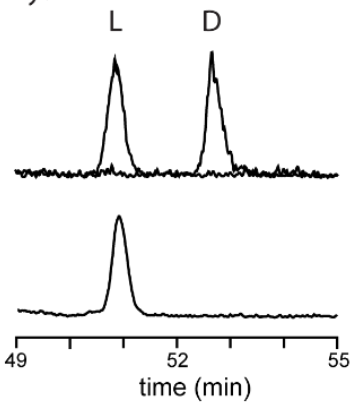

Asp

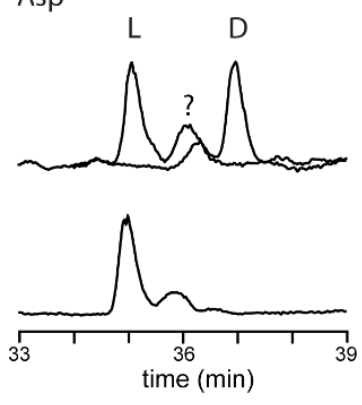

Lys
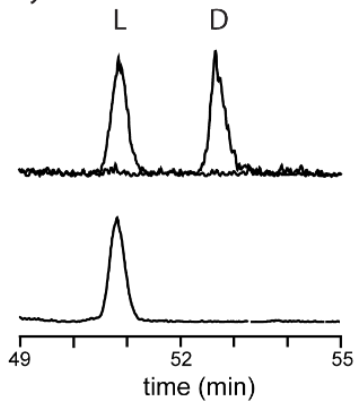

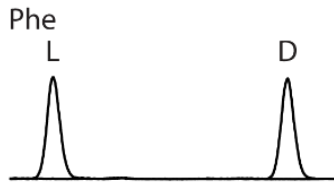

His
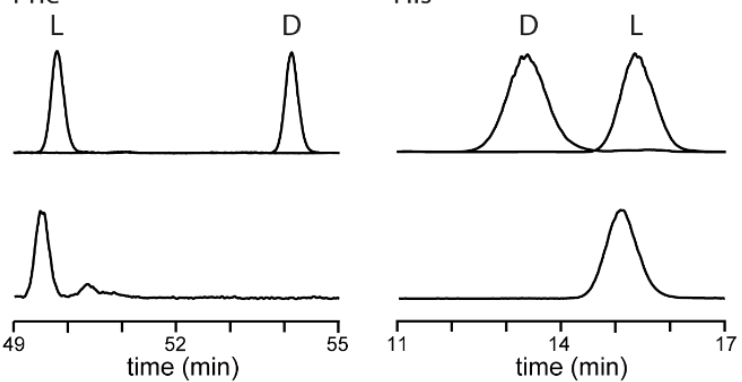

Pro
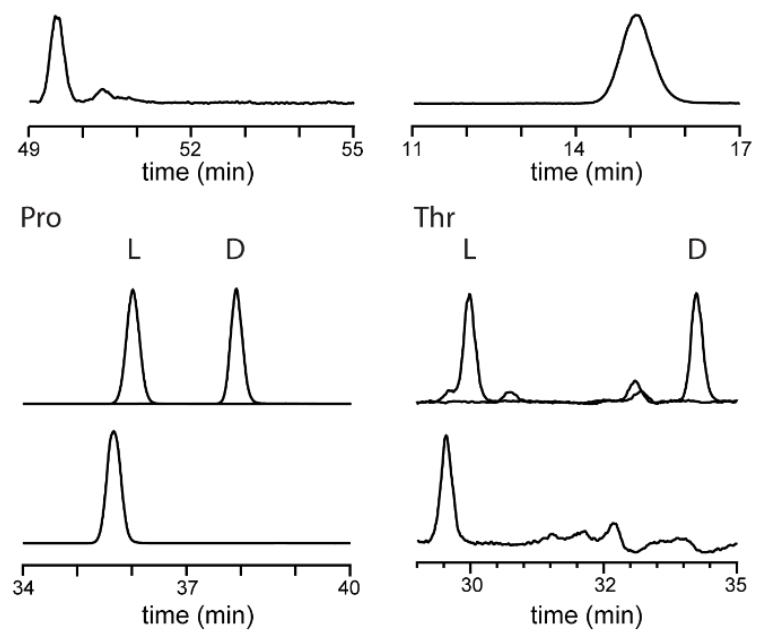

Thr

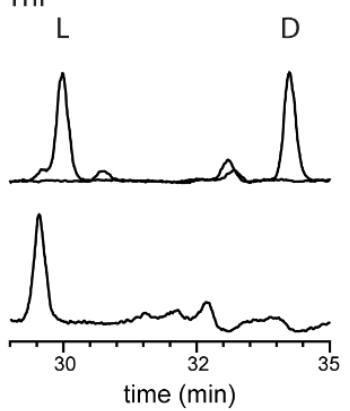

me $(\min )$

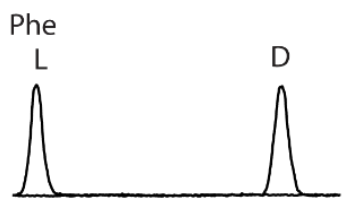

His

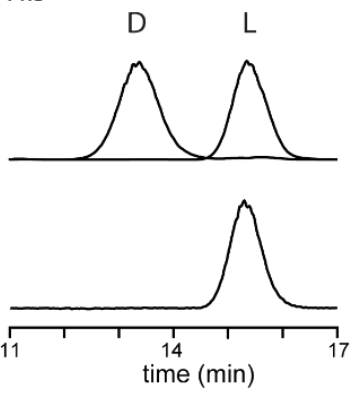

Pro
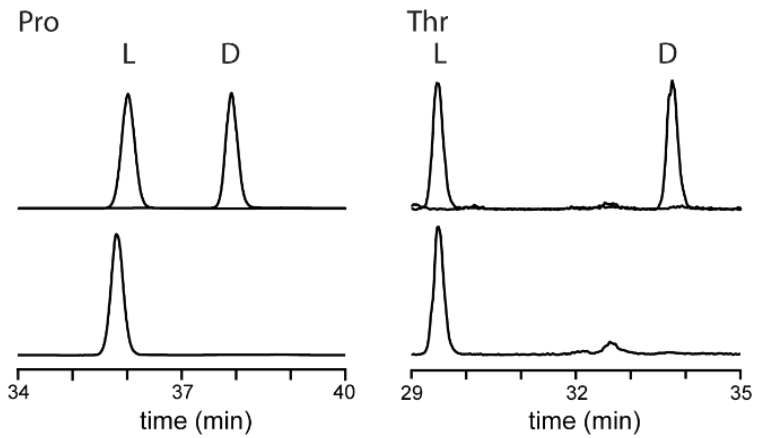
Figure S32. Circular dichroism spectra of thatisin and iso-thatisin. Purified thatisin and isothatisin produce distinct spectra upon analysis by CD spectroscopy. While thatisin has an ellipticity minimum at $207.2 \mathrm{~nm}$, iso-thatisin has ellipticity minima at 204.3 and $205.7 \mathrm{~nm}$. Black arrows in the CD spectra denote the ellipticity minima.

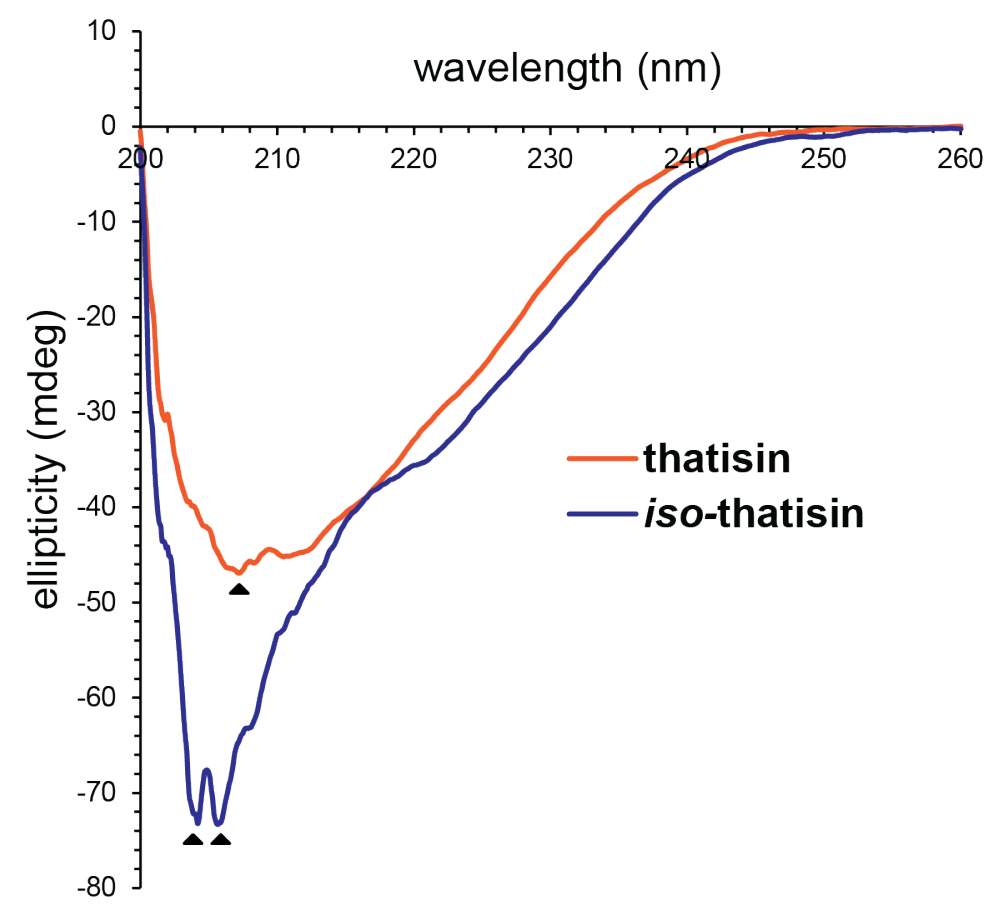


Figure S33. Thatisin conformations based on location of the GPNG bridge. Representative structures for thatisin conformations differing in the position of the GPNG bridge with respect to the plane of the two macrocyles. Peptide backbone of the residues that make up the macrocycles are shown (Thr5 to Asp17); side chains, non-macrocyclized residues (Lys1-Thr4; Pro18-Leu21) and some residue labels have been omitted for clarity. The Thr5-Asp13 macrolactone is teal and the Thr8-Asp17 macrolactone in purple. The GPNG bridge shown in pink can exist above (A) or below (B) the plane of the two macrocycles.

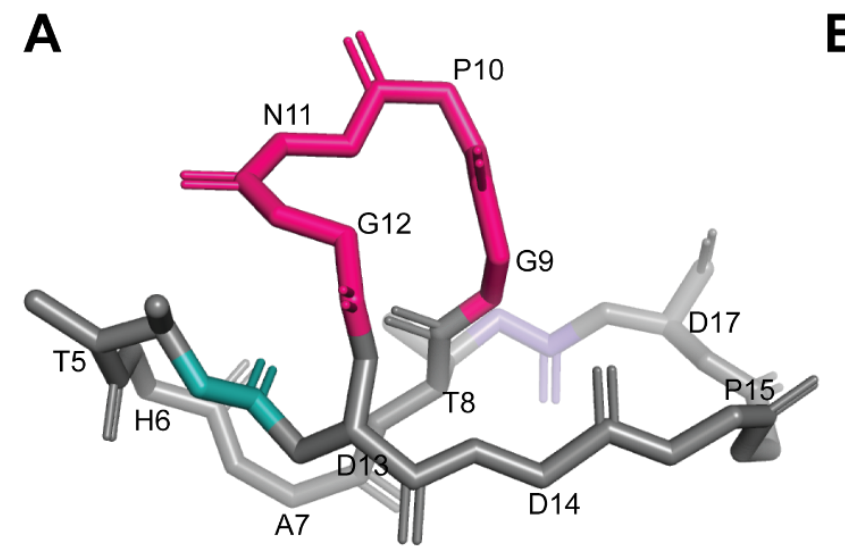

B

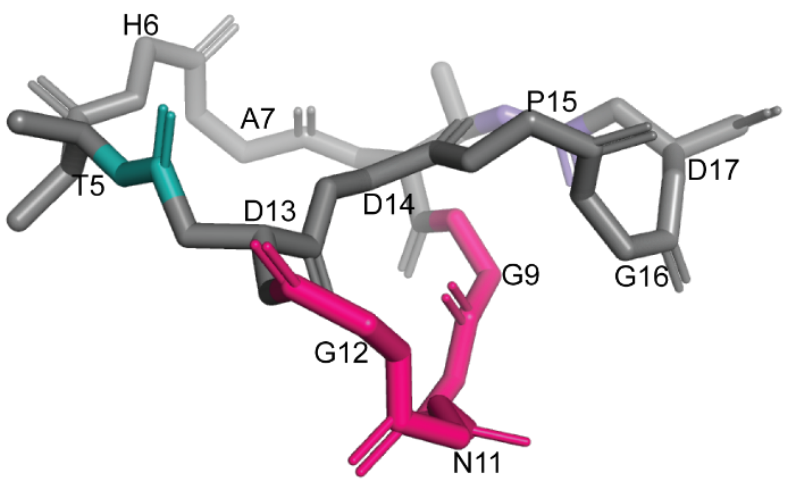


Figure S34. Thatisin and iso-thatisin interconversion. HPLC trace of thatisin (A) and isothatisin (B) after incubation in water at $4{ }^{\circ} \mathrm{C}$ and $25^{\circ} \mathrm{C}$ for $18 \mathrm{~h}$.

A

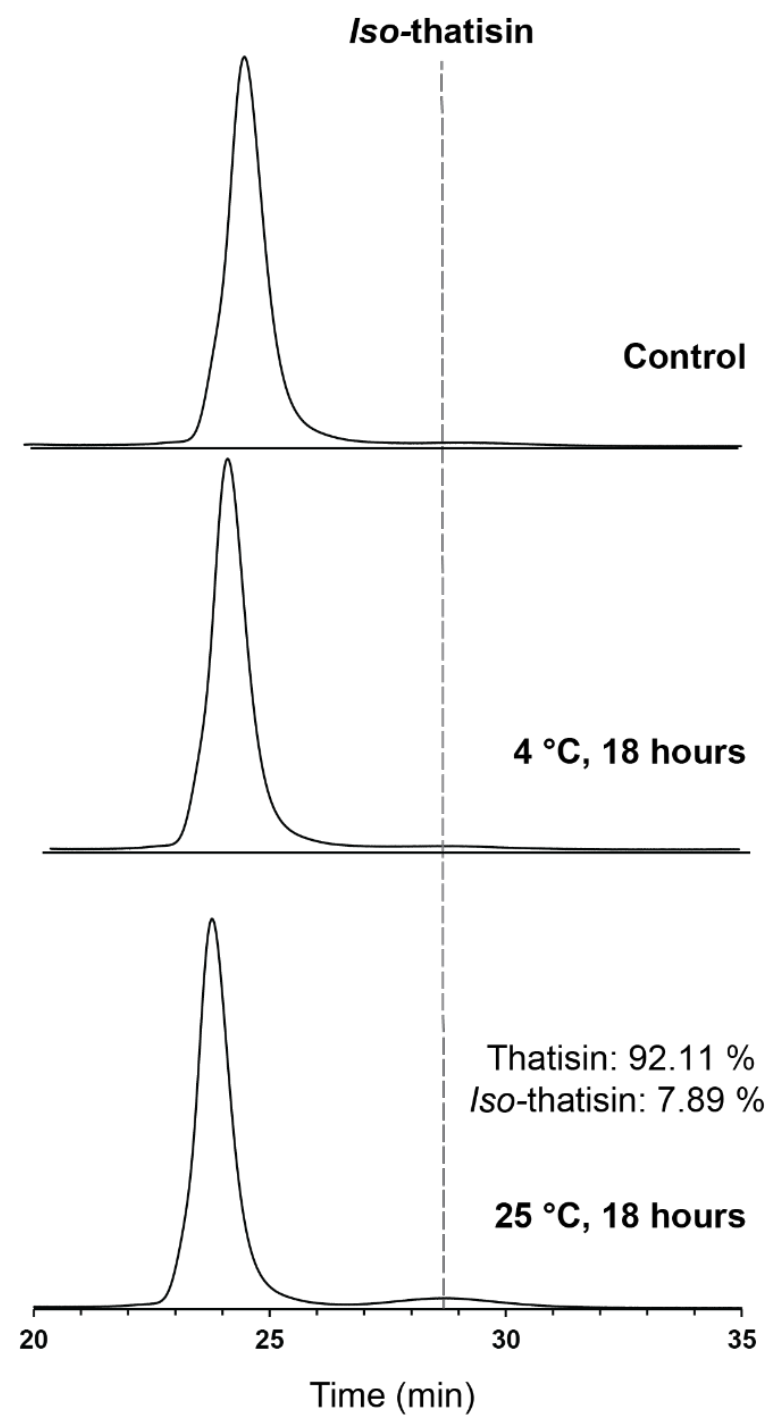

B

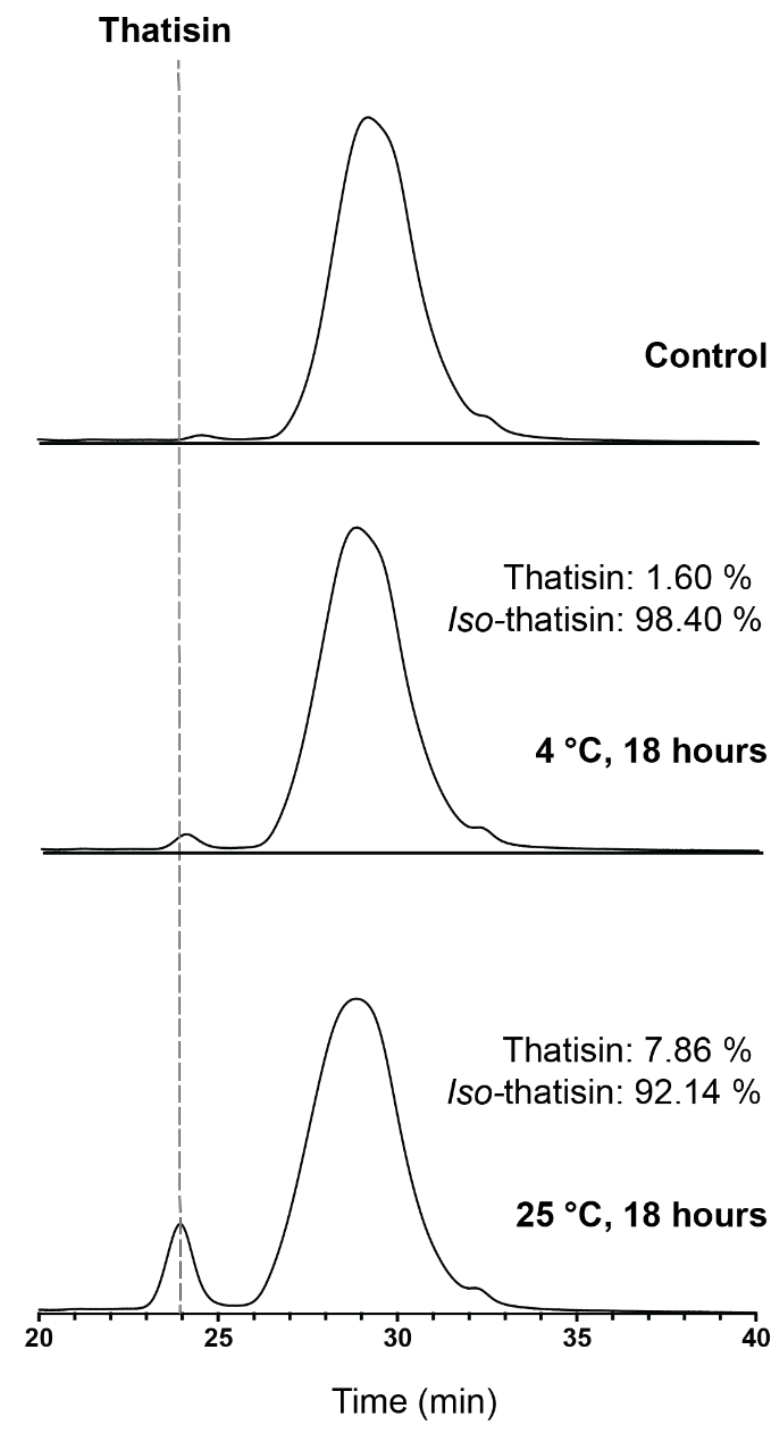


Figure S35. Thatisin: ${ }^{1} \mathbf{H}$ NMR. ${ }^{1} \mathrm{H}$ spectra of thatisin corresponding to the amide $(\mathbf{A})$ and side chain/aliphatic (B) regions upon data collection at $12{ }^{\circ} \mathrm{C}$ at $0 \mathrm{~h}$ (blue) and after $16 \mathrm{~h}$ (orange).
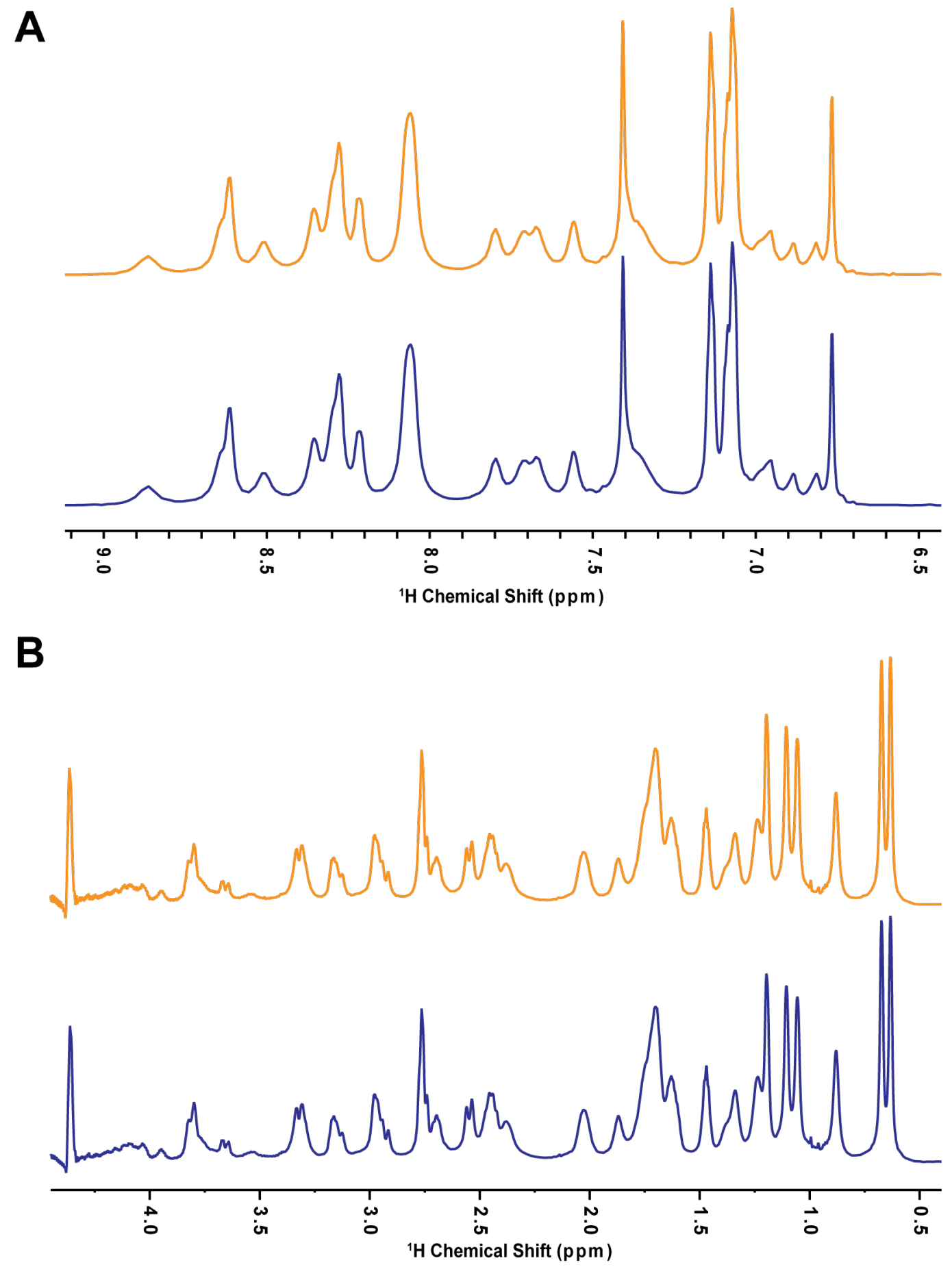
Figure S36. Thatisin: TOCSY NMR. Correlations used to assign individual amino acids in $90 \%$ protiated water are shown in blue with corresponding assignments. All protons were fully assigned based on this data, except Gly2 and Asp19, which were not observed. Leu21 amide resonances were not observed, and only the sidechain could be assigned in deuterated water (Figure S38). Residual HOD signal is indicated in gray.

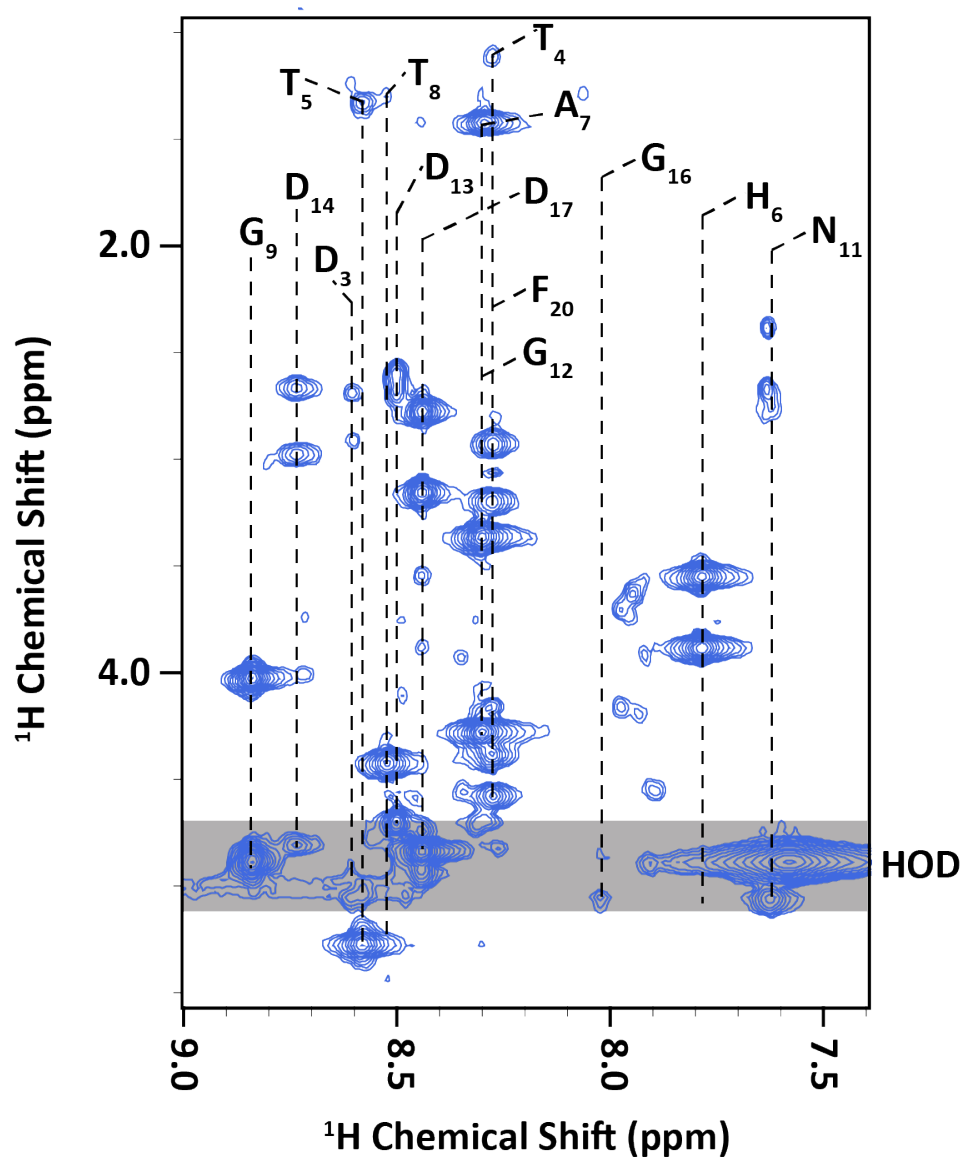


Figure S37. Thatisin: NOESY NMR. (A) Relevant amide-amide NOE contacts used for sequential assignments. Most sequential assignments were accomplished based solely on this experiment. The remaining sequential assignments were accomplished with $\mathrm{H}_{N}-\mathrm{H}_{\alpha}$ and $\mathrm{H}_{\alpha^{-}} \mathrm{H}_{\alpha}$ NOE correlations. His7 and Phe20 sidechain protons were also assigned using indicated NOE correlations. (B) Relevant NOE contacts supporting Thr5-Asp13 and Thr8-Asp17 linkages.

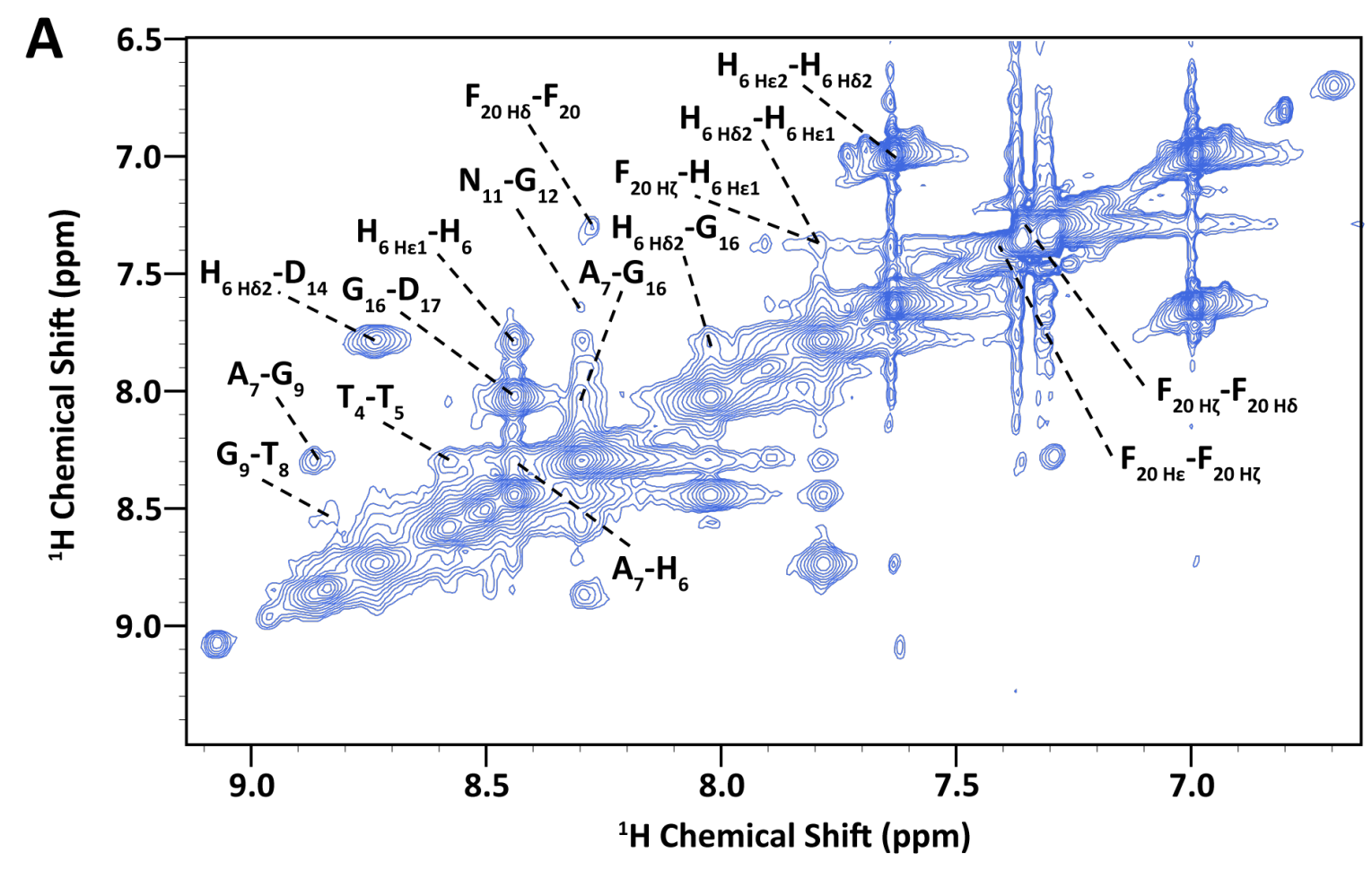

B

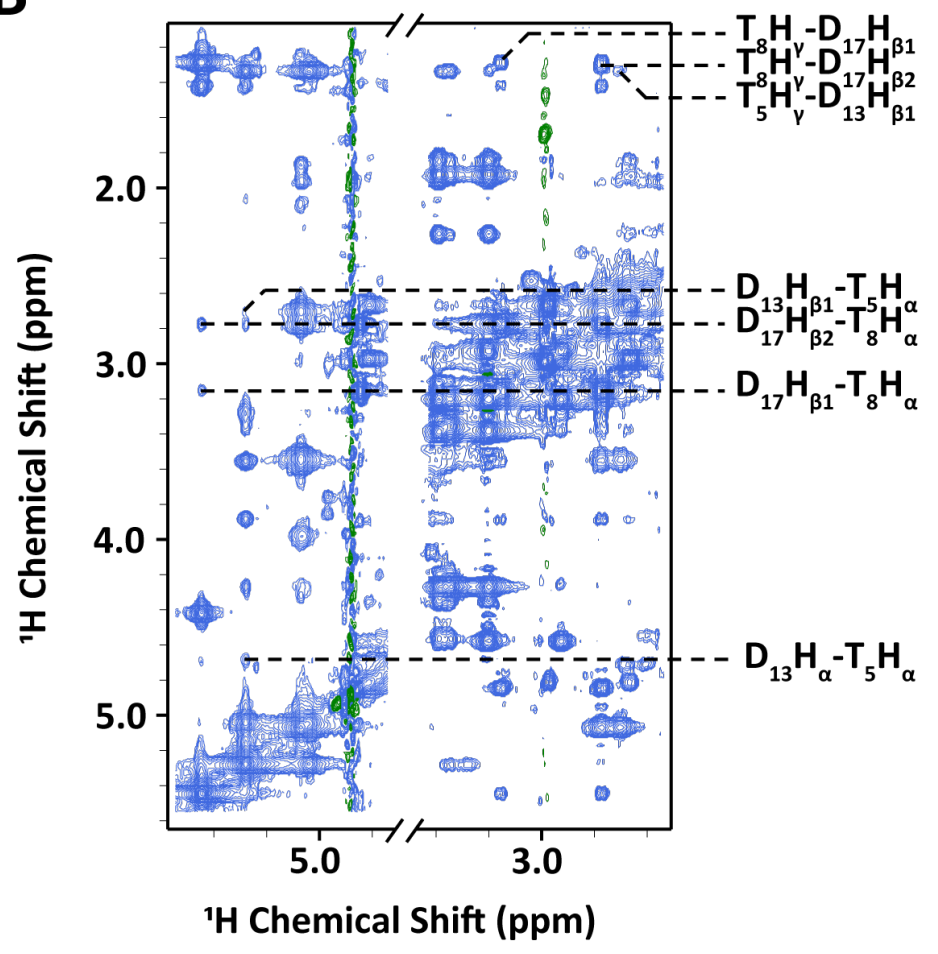


Figure S38. Thatisin: Deuterated Aliphatic TOCSY. Lys1 and Leu21 sidechains were assigned based on characteristic TOCSY fingerprint patterns (left). These residues failed to generate significant NOE correlations, indicating relative flexibility of the $\mathrm{N}$ - and C-terminal fragments of thatisin. Pro10, Pro15, and Pro18 were assigned based on aliphatic TOCSY fingerprints (right) and relevant inter-residue NOE correlations (see Figure S39).
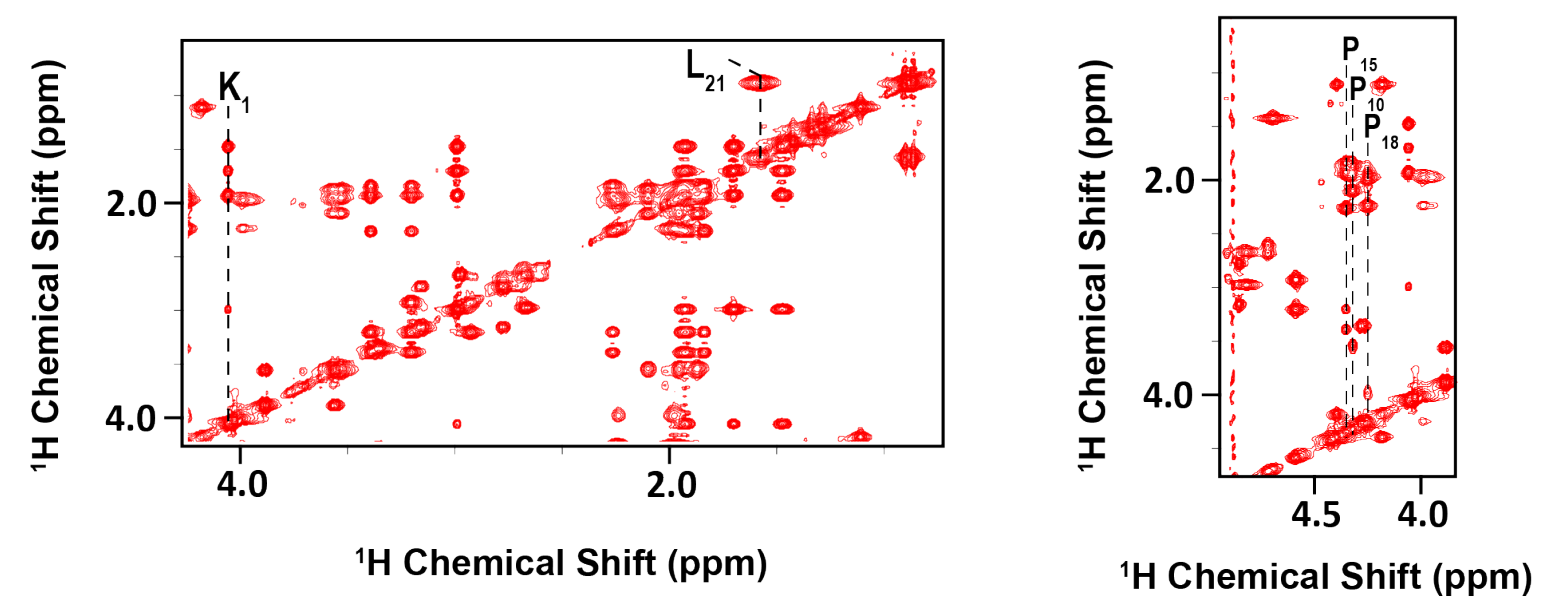
Table S5. NMR chemical shift assignments for thatisin.

\begin{tabular}{|c|c|c|}
\hline Residue & ${ }^{1} \mathrm{H}$ Identity & ${ }^{1} \mathrm{H}$ Chemical Shift \\
\hline Lys1 & $\mathrm{H}_{\alpha}$ & 4.05 \\
\hline Lys1 & $\mathrm{H}_{\beta}$ & 1.93 \\
\hline Lys1 & $\mathrm{H}_{\delta}$ & 1.70 \\
\hline Lys1 & $\mathrm{H}_{\varepsilon}$ & 2.99 \\
\hline Lys1 & $\mathrm{H}_{\mathrm{Y}}$ & 1.47 \\
\hline Asp3 & $\mathrm{H}_{\alpha}$ & 4.91 \\
\hline Asp3 & $\mathrm{H}_{\beta 1}$ & 2.91 \\
\hline Asp3 & $\mathrm{H}_{\beta 2}$ & 2.69 \\
\hline Asp3 & $\mathrm{H}_{\mathrm{N}}$ & 8.60 \\
\hline Thr4 & $\mathrm{H}_{\alpha}$ & 4.39 \\
\hline Thr4 & $\mathrm{H}_{\beta}$ & 4.18 \\
\hline Thr4 & $\mathrm{H}_{\mathrm{Y}}$ & 1.11 \\
\hline Thr4 & $\mathrm{H}_{\alpha}$ & 4.38 \\
\hline Thr5 & $\mathrm{H}_{\alpha}$ & 5.28 \\
\hline Thr5 & $\mathrm{H}_{\beta}$ & 5.05 \\
\hline Thr5 & $\mathrm{H}_{\mathrm{Y}}$ & 1.34 \\
\hline Thr5 & $\mathrm{H}_{\mathrm{N}}$ & 8.58 \\
\hline His6 & $\mathrm{H}_{\alpha}$ & $\mathrm{N} / \mathrm{A}$ \\
\hline His6 & $\mathrm{H}_{\beta 1}$ & 3.89 \\
\hline His6 & $\mathrm{H}_{\beta 2}$ & 3.56 \\
\hline His6 & $\mathrm{H}_{\mathrm{N}}$ & 8.45 \\
\hline His6 & $\mathrm{H}_{\delta 1}$ & 7.64 \\
\hline His6 & $\mathrm{H}_{\delta 2}$ & 7.78 \\
\hline His6 & $\mathrm{H}_{\varepsilon 1}$ & 7.79 \\
\hline His6 & $\mathrm{H}_{\varepsilon 2}$ & 6.99 \\
\hline Ala7 & $\mathrm{H}_{\alpha}$ & 4.70 \\
\hline Ala7 & $\mathrm{H}_{\beta}$ & 1.42 \\
\hline Ala7 & $\mathrm{H}_{\mathrm{N}}$ & 8.32 \\
\hline Thr8 & $\mathrm{H}_{\alpha}$ & 5.45 \\
\hline Thr8 & $\mathrm{H}_{\beta}$ & 4.42 \\
\hline Thr8 & $\mathrm{H}_{\mathrm{Y}}$ & 1.28 \\
\hline Thr8 & $\mathrm{H}_{\mathrm{N}}$ & 8.52 \\
\hline Gly9 & $\mathrm{H}_{\alpha 1}$ & 4.89 \\
\hline Gly9 & $\mathrm{H}_{\mathrm{a} 2}$ & 4.03 \\
\hline Gly9 & $\mathrm{H}_{\mathrm{N}}$ & 8.83 \\
\hline Pro10 & $\mathrm{H}_{\alpha}$ & 4.32 \\
\hline Pro10 & $\mathrm{H}_{\beta 1}$ & 2.10 \\
\hline Pro10 & $\mathrm{H}_{\beta 2}$ & 1.95 \\
\hline Pro10 & $\mathrm{H}_{\delta 1}$ & 3.57 \\
\hline Pro10 & $\mathrm{H}_{\delta 2}$ & 3.53 \\
\hline Pro10 & $\mathrm{H}_{\mathrm{Y}}$ & 1.87 \\
\hline Asn11 & $\mathrm{H}_{\alpha}$ & 5.08 \\
\hline
\end{tabular}

\begin{tabular}{|c|c|c|}
\hline Residue & ${ }^{1} \mathrm{H}$ Identity & ${ }^{1} \mathrm{H}$ Chemical Shift \\
\hline Asn11 & $\mathrm{H}_{\beta 1}$ & 2.78 \\
\hline Asn11 & $\mathrm{H}_{\beta 2}$ & 2.71 \\
\hline Asn11 & $\mathrm{H}_{\mathrm{N}}$ & 7.64 \\
\hline Gly12 & $\mathrm{H}_{\mathrm{a} 1}$ & 4.28 \\
\hline Gly12 & $\mathrm{H}_{\mathrm{\alpha} 2}$ & 3.37 \\
\hline Gly12 & $\mathrm{H}_{\mathrm{N}}$ & 8.30 \\
\hline Asp13 & $\mathrm{H}_{\alpha}$ & 4.71 \\
\hline Asp13 & $\mathrm{H}_{\beta 1}$ & 2.68 \\
\hline Asp13 & $\mathrm{H}_{\beta 2}$ & 2.61 \\
\hline Asp13 & $\mathrm{H}_{\mathrm{N}}$ & 8.50 \\
\hline Asp14 & $\mathrm{H}_{\alpha}$ & 4.82 \\
\hline Asp14 & $\mathrm{H}_{\beta 1}$ & 2.98 \\
\hline Asp14 & $\mathrm{H}_{\beta 2}$ & 2.67 \\
\hline Asp14 & $\mathrm{H}_{\mathrm{N}}$ & 8.74 \\
\hline Pro15 & $\mathrm{Ha}$ & 4.35 \\
\hline Pro15 & $\mathrm{H}_{\beta 1}$ & 2.26 \\
\hline Pro15 & $\mathrm{H}_{\beta 2}$ & 1.93 \\
\hline Pro15 & $\mathrm{H}_{\delta 1}$ & 3.38 \\
\hline Pro15 & $\mathrm{H}_{\delta 2}$ & 3.20 \\
\hline Pro15 & $\mathrm{H}_{\mathrm{y}}$ & 1.84 \\
\hline Gly16 & $\mathrm{H}_{\alpha 1}$ & 5.05 \\
\hline Gly16 & $\mathrm{H}_{\alpha 2}$ & 4.91 \\
\hline Gly16 & $\mathrm{H}_{\mathrm{N}}$ & 8.03 \\
\hline Asp17 & $\mathrm{H}_{\alpha}$ & 4.85 \\
\hline Asp17 & $\mathrm{H}_{\beta 1}$ & 3.16 \\
\hline Asp17 & $\mathrm{H}_{\beta 2}$ & 2.78 \\
\hline Asp17 & $\mathrm{H}_{\mathrm{N}}$ & 8.45 \\
\hline Pro18 & $\mathrm{H}_{\alpha}$ & 4.27 \\
\hline Pro18 & $\mathrm{H}_{\beta}$ & 2.24 \\
\hline Pro18 & $\mathrm{H}_{\delta}$ & 3.98 \\
\hline Pro18 & $\mathrm{H}_{\mathrm{v}}$ & 1.98 \\
\hline Phe20 & $\mathrm{H}_{\alpha}$ & 4.58 \\
\hline Phe20 & $\mathrm{H}_{\beta 1}$ & 3.20 \\
\hline Phe20 & $\mathrm{H}_{\beta 2}$ & 2.93 \\
\hline Phe20 & $\mathrm{H}_{\mathrm{N}}$ & 8.29 \\
\hline Phe20 & $\mathrm{H}_{\varepsilon}$ & 7.47 \\
\hline Phe20 & $\mathrm{H}_{\delta}$ & 7.29 \\
\hline Phe20 & $\mathrm{H}_{\zeta}$ & 7.36 \\
\hline Leu21 & $\mathrm{H}_{\alpha}$ & 4.29 \\
\hline Leu21 & $\mathrm{H}_{\delta 1}$ & 0.89 \\
\hline Leu21 & $\mathrm{H}_{\delta 2}$ & 0.86 \\
\hline Leu21 & $\mathrm{H}_{\mathrm{Y}}$ & 1.57 \\
\hline Leu21 & $\mathrm{H}_{\mathrm{N}}$ & N/A \\
\hline
\end{tabular}


Figure S39. Thatisin: Pro Assignments in deuterated solvent. Relevant NOE correlations used to sequentially assign Pro within thatisin are indicated. The proximity of Gly9 and Asp17 to the water signal prevented observation of inter-residue NOEs to assign the configuration of Pro10 and Pro18.

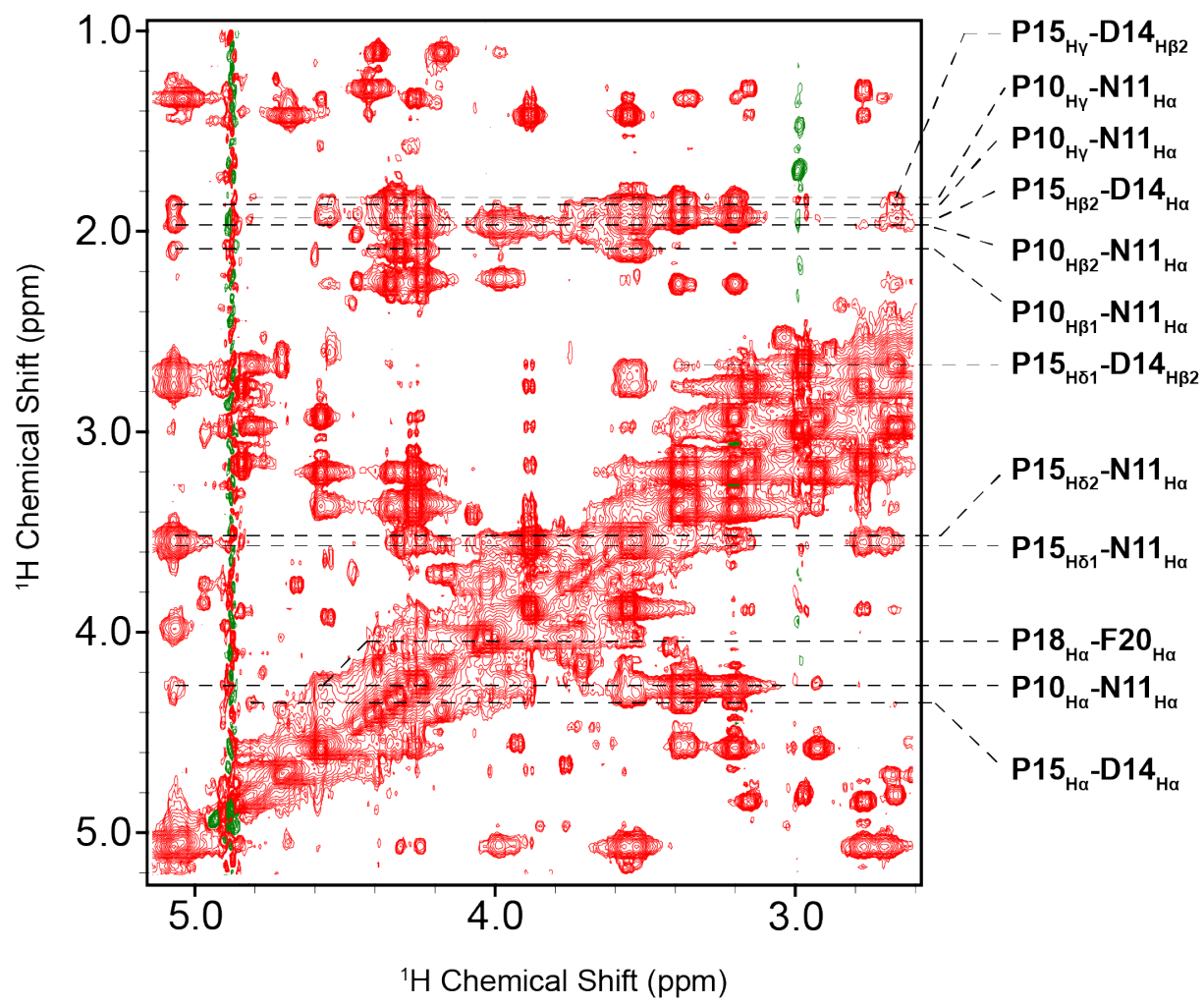


Table S6. Molecular dynamics simulations on 16 possible thatisin conformations.

\begin{tabular}{|c|c|c|}
\hline Thatisin conformation & $\begin{array}{c}\text { Number of } \\
\text { converged structures }\end{array}$ & Average energy (kcal/mol) \\
\hline $\mathrm{GPNG}_{\text {up }} \mathrm{P} 10_{\text {trans }}-\mathrm{P} 15_{\text {trans }}-\mathrm{P} 18_{\text {trans }}$ & 14 & $-619.1 \pm 5.6$ \\
\hline GPNG $_{\text {up }} \mathrm{P} 10_{\text {trans }}-\mathrm{P} 15_{\text {trans }}-\mathrm{P} 18_{\text {cis }}$ & 9 & $-617.7 \pm 5.5$ \\
\hline GPNG up $\mathrm{P} 10_{\text {trans }}-\mathrm{P} 15_{\text {cis }}-\mathrm{P} 18_{\text {trans }}$ & 17 & $-612.5 \pm 7.1$ \\
\hline $\mathrm{GPNG}_{\text {up }} \mathrm{P} 10_{\text {cis }}-\mathrm{P} 15_{\text {trans }}-\mathrm{P} 18_{\text {trans }}$ & 1 & -607.4 \\
\hline $\mathrm{GPNG}_{\text {up }} \mathrm{P} 10_{\text {trans }}-\mathrm{P} 15_{\text {cis }}-\mathrm{P} 18_{\text {cis }}$ & 1 & -614.7 \\
\hline $\mathrm{GPNG}_{\text {up }} \mathrm{P} 10_{\text {cis }}-\mathrm{P} 15_{\text {trans }}-\mathrm{P} 18_{\text {cis }}$ & 2 & $-613.4 \pm 0.1$ \\
\hline $\mathrm{GPNG}_{\text {up }} \mathrm{P} 10_{\text {cis }}-\mathrm{P} 15_{\text {cis }}-\mathrm{P} 18_{\text {trans }}$ & 3 & $-611.5 \pm 4.1$ \\
\hline $\mathrm{GPNG}_{\text {up }} \mathrm{P} 10_{c i s}-\mathrm{P} 15_{\text {cis }}-\mathrm{P} 18_{\text {cis }}$ & 0 & Not available \\
\hline $\mathrm{GPNG}_{\text {down }} \mathrm{P} 10_{\text {trans }}-\mathrm{P} 15_{\text {trans }}-\mathrm{P} 18_{\text {trans }}$ & 20 & $-627.4 \pm 3.5$ \\
\hline GPNG down $_{\text {P10trans }}-\mathrm{P} 15_{\text {trans }}-\mathrm{P} 18_{\text {cis }}$ & 10 & $-622.7 \pm 5.2$ \\
\hline $\mathrm{GPNG}_{\text {down }} \mathrm{P} 10_{\text {trans }}-\mathrm{P} 15_{\text {cis }}-\mathrm{P} 18_{\text {trans }}$ & 5 & $-626.4 \pm 5.4$ \\
\hline $\mathrm{GPNG}_{\text {down }} \mathrm{P} 10_{\text {cis }}-\mathrm{P} 15_{\text {trans }}-\mathrm{P} 18_{\text {trans }}$ & 4 & $-622.1 \pm 5.0$ \\
\hline $\mathrm{GPNG}_{\text {down }} \mathrm{P} 10_{\text {trans }}-\mathrm{P} 15_{\text {cis }}-\mathrm{P} 18_{\text {cis }}$ & 0 & Not available \\
\hline $\mathrm{GPNG}_{\text {down }} \mathrm{P} 10_{\text {cis }}-\mathrm{P} 15_{\text {trans }}-\mathrm{P} 18_{\text {cis }}$ & 0 & Not available \\
\hline $\mathrm{GPNG}_{\text {down }} \mathrm{P} 10_{\text {cis }}-\mathrm{P} 15_{\text {cis }}-\mathrm{P} 18_{\text {trans }}$ & 0 & Not available \\
\hline $\mathrm{GPNG}_{\text {down }} \mathrm{P} 10_{c i s}-\mathrm{P} 15_{c i s}-\mathrm{P} 18_{c i s}$ & 0 & Not available \\
\hline
\end{tabular}


Table S7. Solvent-accessible surface area for GPNG $_{\text {up }} \mathbf{P} 10_{\text {trans }}-\mathrm{P} 15_{\text {trans }}-\mathrm{P} 18_{\text {trans }}$ structures. Fraction represents the total number of structures with a SASA greater than $0 \AA^{2}$.

\begin{tabular}{ccc}
\hline \multirow{2}{*}{ Structure } & \multicolumn{2}{c}{ SASA for carbonyl carbon $\left(\AA^{2}\right)$} \\
& Thr5-Asp13 macrolactone & Thr8-Asp17 macrolactone \\
\hline 1 & 4.82 & 0.00 \\
2 & 2.41 & 0.00 \\
3 & 3.44 & 0.00 \\
4 & 0.00 & 0.34 \\
5 & 0.00 & 0.00 \\
6 & 0.00 & 0.00 \\
7 & 0.00 & 0.00 \\
8 & 2.75 & 0.00 \\
9 & 0.00 & 0.34 \\
10 & 0.00 & 0.00 \\
11 & 0.00 & 0.00 \\
12 & 0.00 & 0.00 \\
13 & 0.00 & 0.00 \\
14 & 0.00 & 0.00 \\
Fraction & $4 / 14$ & $2 / 14$ \\
\hline
\end{tabular}


Table S8. Solvent-accessible surface area for GPNG $_{\text {up }} \mathbf{P 1 0}$ trans $_{\text {tr }}-\mathrm{P} 15_{\text {cis }}-\mathrm{P} 18_{\text {trans }}$ structures. Fraction represents the total number of structures with a SASA greater than $0 \AA^{2}$.

\begin{tabular}{ccc}
\hline \multirow{2}{*}{ Structure } & \multicolumn{2}{c}{ SASA for carbonyl carbon $\left(\AA^{2}\right)$} \\
& Thr5-Asp13 macrolactone & Thr8-Asp17 macrolactone \\
\hline 1 & 0.00 & 0.00 \\
2 & 0.00 & 0.00 \\
3 & 0.00 & 0.00 \\
4 & 0.00 & 0.00 \\
5 & 0.00 & 0.00 \\
6 & 0.00 & 2.06 \\
7 & 0.00 & 0.69 \\
8 & 0.00 & 0.69 \\
9 & 0.00 & 3.10 \\
10 & 0.00 & 1.03 \\
11 & 0.00 & 3.10 \\
12 & 3.10 & 0.00 \\
13 & 0.00 & 3.10 \\
14 & 1.72 & 2.75 \\
15 & 0.00 & 2.06 \\
16 & 0.00 & 2.41 \\
17 & 0.00 & 2.41 \\
Fraction & $2 / 17$ & $11 / 17$ \\
\hline
\end{tabular}




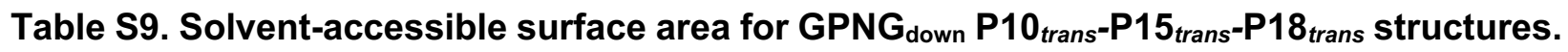
Fraction represents the total number of structures with SASA greater than $0 \AA^{2}$.

\begin{tabular}{|c|c|c|}
\hline \multirow{2}{*}{ Structure } & \multicolumn{2}{|c|}{ SASA for carbonyl carbon $\left(\AA^{2}\right)$} \\
\hline & Thr5-Asp13 macrolactone & Thr8-Asp17 macrolactone \\
\hline 1 & 5.51 & 0.00 \\
\hline 2 & 0.00 & 3.10 \\
\hline 3 & 0.00 & 0.00 \\
\hline 4 & 3.10 & 4.13 \\
\hline 5 & 0.34 & 1.03 \\
\hline 6 & 1.03 & 0.00 \\
\hline 7 & 1.38 & 0.00 \\
\hline 8 & 0.00 & 0.00 \\
\hline 9 & 0.00 & 1.72 \\
\hline 10 & 0.00 & 0.00 \\
\hline 11 & 1.38 & 1.72 \\
\hline 12 & 0.00 & 0.00 \\
\hline 13 & 0.69 & 0.00 \\
\hline 14 & 0.00 & 0.00 \\
\hline 15 & 2.06 & 0.00 \\
\hline 16 & 0.00 & 0.00 \\
\hline 17 & 0.00 & 0.00 \\
\hline 18 & 2.41 & 0.00 \\
\hline 19 & 0.00 & 2.41 \\
\hline 20 & 0.00 & 0.00 \\
\hline Fraction & $9 / 20$ & $6 / 20$ \\
\hline
\end{tabular}


Table S10. Solvent-accessible surface area for $\mathrm{GPNG}_{\text {down }} \mathbf{P} \mathbf{1 0}_{\text {trans }}-\mathrm{P} 15_{\text {trans }}-\mathrm{P} 18_{\text {cis }}$ structures. Fraction represents the total number of structures with a SASA greater than $0 \AA^{2}$.

\begin{tabular}{ccc}
\hline \multirow{2}{*}{ Structure } & \multicolumn{2}{c}{ SASA for carbonyl carbon $\left(\AA^{2}\right)$} \\
& Thr5-Asp13 macrolactone & Thr8-Asp17 macrolactone \\
\hline 1 & 0.00 & 0.00 \\
2 & 1.03 & 0.00 \\
3 & 1.38 & 0.00 \\
4 & 0.00 & 0.00 \\
5 & 0.00 & 0.00 \\
6 & 0.00 & 0.00 \\
7 & 0.00 & 0.00 \\
8 & 0.00 & 0.00 \\
9 & 0.00 & 0.00 \\
10 & 0.00 & 0.00 \\
Fraction & $2 / 10$ & $0 / 10$ \\
\hline
\end{tabular}


Table S11. Oligonucleotide primers used in this study.

\begin{tabular}{|l|l|}
\hline Primer & Nucleotide sequence (5' $\boldsymbol{\rightarrow}$ 3') \\
\hline pETDuetK-ThtA-Insert-F & GAGAACCTGTACTTCCAATCCATGAGTAAGCAAGACGTTTACAGCAACAACGC \\
\hline pETDuetK-ThtA-Insert-R & CTGTTCGACTTAAGCATTATGCTTTACAGGAAATCGGGATCGCCCGGA \\
\hline pETDuetK-ThtA-Vector-F & TCCGGGCGATCCCGATTTCCTGTAAAGCATAATGCTTAAGTCGAACAG \\
\hline pETDuetK-ThtA-Vector-R & GCGTTGTTGCTGTAAACGTCTTGCTTACTCATGGATTGGAAGTACAGGTTCTC \\
\hline pETDuetK-ThtB-Insert-F & GTATATTAGTTAAGTATAAGAAGGAGATATACATATGCCCGCTCACCGCCGTC \\
\hline pETDuetK-ThtB-Insert-R & CCGATATCCAATTGAGATCTGCCTACTCCAGCGTAATCCCCGCCG \\
\hline pETDuetK-ThtB-Vector-F & CGGCGGGGATTACGCTGGAGTAGGCAGATCTCAATTGGATATCGG \\
\hline pETDuetK-ThtB-Vector-R & GACGGCGGTGAGCGGGCATATGTATATCTCCTTCTTATACTTAACTAATATAC \\
\hline ThtA-AllMutants-backbone-F & GCATCGACCTGGCCATCGACGC \\
\hline ThtA-AllMutants-insert-R & GCGTCGATGGCCAGGTCGATGC \\
\hline ThtA-D3A-insert-F & GGCAAGGGCGCTACCACCCAC \\
\hline ThtA-D3A-backbone-R & GTGGGTGGTAGCGCCCTTGCC \\
\hline ThtA-T4A-insert-F & CAAGGGCGATGCCACCCACG \\
\hline ThtA-T4A-backbone-R & CGTGGGTGGCATCGCCCTTG \\
\hline ThtA-T5A-insert-F & GGGCGATACCGCCCACGCCAC \\
\hline ThtA-T5A-backbone-R & GTGGCGTGGGCGGTATCGCCC \\
\hline ThtA-T8A-insert-F & CACGCCGCCGGCCCGAAC \\
\hline ThtA-T8A-backbone-R & GTTCGGGCCGGCGGCGTG \\
\hline ThtA-D13A-insert-F & CCGAACGGCGCCGATCCG \\
\hline ThtA-D13A-backbone-R & CGGATCGGCGCCGTTCGG \\
\hline ThtA-D14A-insert-F & CGAACGGCGACGCTCCGGG \\
\hline ThtA-D14A-backbone-R & CCCGGAGCGTCGCCGTTCG \\
\hline ThtA-D17A-insert-F & GATCCGGGCGCTCCCGATTTC \\
\hline ThtA-D17A-backbone-R & GAAATCGGGAGCGCCCGGATC \\
\hline ThtA-D19A-insert-F & GGGCGATCCCGCTTTCCTGTAAAG \\
\hline ThtA-D19A-backbone-R & CTTTACAGGAAAGCGGGATCGCCC \\
\hline
\end{tabular}




\section{Supplemental References.}

(1) Tietz, J. I.; Schwalen, C. J.; Patel, P. S.; Maxson, T.; Blair, P. M.; Tai, H.-C.; Zakai, U. I.; Mitchell, D. A. A New Genome-Mining Tool Redefines the Lasso Peptide Biosynthetic Landscape. Nat. Chem. Biol. 2017, 13 (5), 470-478. https://doi.org/10.1038/nchembio.2319.

(2) Hudson, G. A.; Burkhart, B. J.; DiCaprio, A. J.; Schwalen, C. J.; Kille, B.; Pogorelov, T. V.; Mitchell, D. A. Bioinformatic Mapping of Radical S-Adenosylmethionine-Dependent Ribosomally Synthesized and Post-Translationally Modified Peptides Identifies New Ca, C $\beta$, and Cy-Linked Thioether-Containing Peptides. J. Am. Chem. Soc. 2019, 141 (20), 82288238. https://doi.org/10.1021/jacs.9b01519.

(3) Walker, M. C.; Eslami, S. M.; Hetrick, K. J.; Ackenhusen, S. E.; Mitchell, D. A.; van der Donk, W. A. Precursor Peptide-Targeted Mining of More than One Hundred Thousand Genomes Expands the Lanthipeptide Natural Product Family. BMC Genomics 2020, 21 (1), 387. https://doi.org/10.1186/s12864-020-06785-7.

(4) Schwalen, C. J.; Hudson, G. A.; Kille, B.; Mitchell, D. A. Bioinformatic Expansion and Discovery of Thiopeptide Antibiotics. J. Am. Chem. Soc. 2018, 140 (30), 9494-9501. https://doi.org/10.1021/jacs.8b03896.

(5) Georgiou, M. A.; Dommaraju, S. R.; Guo, X.; Mast, D. H.; Mitchell, D. A. Bioinformatic and Reactivity-Based Discovery of Linaridins. ACS Chem. Biol. 2020, 15 (11), 2976-2985. https://doi.org/10.1021/acschembio.0c00620.

(6) Kloosterman, A. M.; Shelton, K. E.; van Wezel, G. P.; Medema, M. H.; Mitchell, D. A. RREFinder: A Genome-Mining Tool for Class-Independent RiPP Discovery. mSystems 2020, 5 (5), e00267-20. https://doi.org/10.1128/mSystems.00267-20.

(7) Iyer, L. M.; Abhiman, S.; Maxwell Burroughs, A.; Aravind, L. Amidoligases with ATP-Grasp, Glutamine Synthetase-like and Acetyltransferase-like Domains: Synthesis of Novel Metabolites and Peptide Modifications of Proteins. Mol. Biosyst. 2009, 5 (12), 1636-1660. https://doi.org/10.1039/b917682a.

(8) Lee, H.; Choi, M.; Park, J.-U.; Roh, H.; Kim, S. Genome Mining Reveals High Topological Diversity of $\omega$-Ester-Containing Peptides and Divergent Evolution of ATP-Grasp Macrocyclases. J. Am. Chem. Soc. 2020, 142 (6), 3013-3023. https://doi.org/10.1021/jacs.9b12076.

(9) Bailey, T. L.; Boden, M.; Buske, F. A.; Frith, M.; Grant, C. E.; Clementi, L.; Ren, J.; Li, W. W.; Noble, W. S. MEME SUITE: Tools for Motif Discovery and Searching. Nucleic Acids Res. 2009, 37 (Web Server), W202-W208. https://doi.org/10.1093/nar/gkp335.

(10) Mistry, J.; Finn, R. D.; Eddy, S. R.; Bateman, A.; Punta, M. Challenges in Homology Search: HMMER3 and Convergent Evolution of Coiled-Coil Regions. Nucleic Acids Res. 2013, 41 (12), e121-e121. https://doi.org/10.1093/nar/gkt263.

(11) Heger, A.; Holm, L. Rapid Automatic Detection and Alignment of Repeats in Protein Sequences. Proteins 2000, 41 (2), 224-237. https://doi.org/10.1002/10970134(20001101)41:2<224::aid-prot70>3.0.co;2-z.

(12) Altschul, S. F.; Madden, T. L.; Schaffer, A. A.; Zhang, J.; Zhang, Z.; Miller, W.; Lipman, D. J. Gapped BLAST and PSI-BLAST: A New Generation of Protein Database Search Programs. Nucleic Acids Res. 1997, 25 (17), 3389-3402.

(13) Zallot, R.; Oberg, N.; Gerlt, J. A. The EFI Web Resource for Genomic Enzymology Tools: Leveraging Protein, Genome, and Metagenome Databases to Discover Novel Enzymes and Metabolic Pathways. Biochemistry 2019, $58 \quad$ (41), 4169-4182. https://doi.org/10.1021/acs.biochem.9b00735.

(14) Zallot, R.; Oberg, N. O.; Gerlt, J. A. 'Democratized' Genomic Enzymology Web Tools for Functional Assignment. Curr. Opin. Chem. Biol. 2018, 47, 77-85. https://doi.org/10.1016/j.cbpa.2018.09.009. 
(15) Shannon, P.; Markiel, A.; Ozier, O.; Baliga, N. S.; Wang, J. T.; Ramage, D.; Amin, N.; Schwikowski, B.; Ideker, T. Cytoscape: A Software Environment for Integrated Models of Biomolecular Interaction Networks. Genome Res. 2003, 13 (11), 2498-2504. https://doi.org/10.1101/gr.1239303.

(16) Suzek, B. E.; Wang, Y.; Huang, H.; McGarvey, P. B.; Wu, C. H.; the UniProt Consortium. UniRef Clusters: A Comprehensive and Scalable Alternative for Improving Sequence Similarity Searches. Bioinformatics 2015, $31 \quad$ (6), 926-932. https://doi.org/10.1093/bioinformatics/btu739.

(17) Katoh, K.; Standley, D. M. MAFFT Multiple Sequence Alignment Software Version 7: Improvements in Performance and Usability. Mol. Biol. Evol. 2013, 30 (4), 772-780. https://doi.org/10.1093/molbev/mst010.

(18) Price, M. N.; Dehal, P. S.; Arkin, A. P. FastTree 2 - Approximately Maximum-Likelihood Trees for Large Alignments. PloS One 2010, 5 (3), e9490. https://doi.org/10.1371/journal.pone.0009490.

(19) Letunic, I.; Bork, P. Interactive Tree Of Life (ITOL) v4: Recent Updates and New Developments. Nucleic Acids Res. 2019, 47 (W1), W256-W259. https://doi.org/10.1093/nar/gkz239.

(20) Crooks, G. E. WebLogo: A Sequence Logo Generator. Genome Res. 2004, 14 (6), 11881190. https://doi.org/10.1101/gr.849004.

(21) Bobeica, S. C.; Dong, S.-H.; Huo, L.; Mazo, N.; McLaughlin, M. I.; Jiménez-Osés, G.; Nair, S. K.; van der Donk, W. A. Insights into AMS/PCAT Transporters from Biochemical and Structural Characterization of a Double Glycine Motif Protease. Elife 2019, 8. https://doi.org/10.7554/eLife.42305.

(22) Delaglio, F.; Grzesiek, S.; Vuister, GeertenW.; Zhu, G.; Pfeifer, J.; Bax, A. NMRPipe: A Multidimensional Spectral Processing System Based on UNIX Pipes. J. Biomol. NMR 1995, 6 (3). https://doi.org/10.1007/BF00197809.

(23) Lee, W.; Tonelli, M.; Markley, J. L. NMRFAM-SPARKY: Enhanced Software for Biomolecular NMR Spectroscopy. Bioinformatics 2015, $31 \quad$ (8), 1325-1327. https://doi.org/10.1093/bioinformatics/btu830.

(24) Molecular Operating Environment (MOE), 2019.01.; Chemical Computing Group ULC: 1010 Sherbooke St. West, Suite \#910, Montreal, QC, Canada, H3A 2R7, 2021.

(25) Case, D.; Babin, V.; Berryman, J.; Betz, R.; Cai, Q.; Cerutti, D.; Cheatham, T.; Darden, T.; Duke, R.; Gohlke, H.; et al. Amber 2014; 2014.

(26) Labute, P. The Generalized Born/Volume Integral Implicit Solvent Model: Estimation of the Free Energy of Hydration Using London Dispersion Instead of Atomic Surface Area. J. Comput. Chem. 2008, 29 (10), 1693-1698. https://doi.org/10.1002/jcc.20933.

(27) Labute, P. LowModeMD--Implicit Low-Mode Velocity Filtering Applied to Conformational Search of Macrocycles and Protein Loops. J. Chem. Inf. Model. 2010, 50 (5), 792-800. https://doi.org/10.1021/ci900508k.

(28) Humphrey, W.; Dalke, A.; Schulten, K. VMD: Visual Molecular Dynamics. J. Mol. Graph. 1996, 14 (1), 33-38. https://doi.org/10.1016/0263-7855(96)00018-5.

(29) Haft, D. H.; Selengut, J. D.; Richter, R. A.; Harkins, D.; Basu, M. K.; Beck, E. TIGRFAMs and Genome Properties in 2013. Nucleic Acids Res. 2012, 41 (D1), D387-D395. https://doi.org/10.1093/nar/gks1234.

(30) Mitchell, A. L.; Attwood, T. K.; Babbitt, P. C.; Blum, M.; Bork, P.; Bridge, A.; Brown, S. D.; Chang, H.-Y.; El-Gebali, S.; Fraser, M. I.; et al. InterPro in 2019: Improving Coverage, Classification and Access to Protein Sequence Annotations. Nucleic Acids Res. 2019, 47 (D1), D351-D360. https://doi.org/10.1093/nar/gky1100.

(31) Madeira, F.; Park, Y. mi; Lee, J.; Buso, N.; Gur, T.; Madhusoodanan, N.; Basutkar, P.; Tivey, A. R. N.; Potter, S. C.; Finn, R. D.; et al. The EMBL-EBI Search and Sequence Analysis 
Tools APIs in 2019. Nucleic Acids Res. 2019, 47 (W1), W636-W641. https://doi.org/10.1093/nar/gkz268.

(32) Mahanta, N.; Szantai-Kis, D. M.; Petersson, E. J.; Mitchell, D. A. Biosynthesis and Chemical Applications of Thioamides. ACS Chem. Biol. 2019, 14 (2), 142-163. https://doi.org/10.1021/acschembio.8b01022.

(33) Liu, A.; Si, Y.; Dong, S.-H.; Mahanta, N.; Penkala, H. N.; Nair, S. K.; Mitchell, D. A. Functional Elucidation of TfuA in Peptide Backbone Thioamidation. Nat. Chem. Biol. 2021, 17 (5), 585592. https://doi.org/10.1038/s41589-021-00771-0.

(34) Montalbán-López, M.; Scott, T. A.; Ramesh, S.; Rahman, I. R.; van Heel, A. J.; Viel, J. H.; Bandarian, V.; Dittmann, E.; Genilloud, O.; Goto, Y.; et al. New Developments in RiPP Discovery, Enzymology and Engineering. Nat. Prod. Rep. 2021, 38, 130-239. https://doi.org/10.1039/D0NP00027B.

(35) Boeckmann, B. The SWISS-PROT Protein Knowledgebase and Its Supplement TrEMBL in 2003. Nucleic Acids Res. 2003, 31 (1), 365-370. https://doi.org/10.1093/nar/gkg095. 